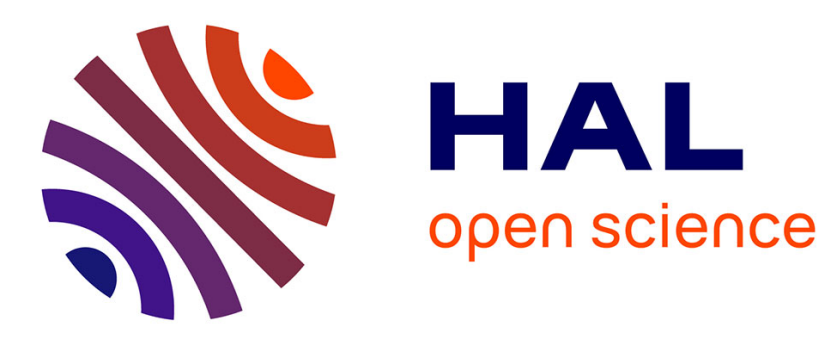

\title{
Sociétés, environnements, santé
}

Nicole Vernazza-Licht, Marc-Eric Gruénais, Daniel Bley

\section{To cite this version:}

Nicole Vernazza-Licht, Marc-Eric Gruénais, Daniel Bley (Dir.). Sociétés, environnements, santé. IRD

Éditions, pp.364, 2010, Collection Objectifs Sud / IRD, 978-2-7099-1694-3. hal-01292917

\section{HAL Id: hal-01292917 \\ https://hal.science/hal-01292917}

Submitted on 27 Mar 2017

HAL is a multi-disciplinary open access archive for the deposit and dissemination of scientific research documents, whether they are published or not. The documents may come from teaching and research institutions in France or abroad, or from public or private research centers.
L'archive ouverte pluridisciplinaire HAL, est destinée au dépôt et à la diffusion de documents scientifiques de niveau recherche, publiés ou non, émanant des établissements d'enseignement et de recherche français ou étrangers, des laboratoires publics ou privés. 


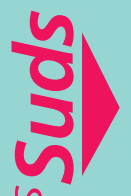

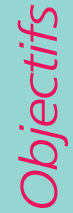
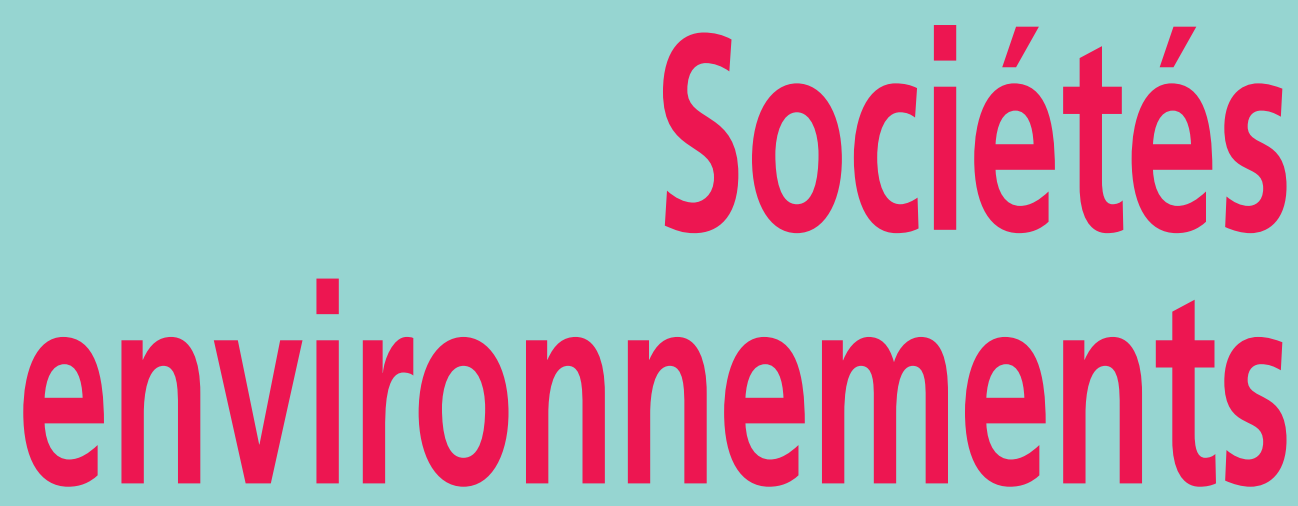

$$
\text { santé }
$$

Sous la direction de

Nicole Vernazza-Licht Marc-Éric GruÉNAIS Daniel BLEY

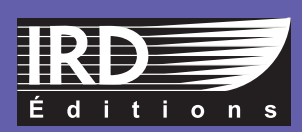


Sociétés,

environnements, santé 


\section{Objectifs Suds}

Les défis du développement

Collection généraliste consacrée aux grandes questions contemporaines relatives au développement et à l'environnement. $A$ travers des synthèses ou des éclairages originaux, elle rend compte des recherches pluridisciplinaires menées par l'IRD en partenariat avec les pays du Sud pour répondre aux défis de la mondialisation et mettre en œuvre les conditions du codéveloppement.

L'IRD souhaite ainsi répondre aux attentes d'un large public en lui offrant le résultat des réflexions des chercheurs et en l'informant de manière rigoureuse sur les grands enjeux contemporains.

Directeur de collection : Benoît Antheaume

benoit.antheaume@ird.fr

Derniers volumes parus :

Migrants des Suds

V. Baby-Collin, G. Cortes, L. Faret, H. Guétat-Bernard

Aires protégées, espaces durables?

C. AUBERTIN, E. RODARY (éd.)

Les marchés de la biodiversité

C. AUBERTIN, F. PINTON, V. BOISVERT (éd.)

Le monde peut-il nourrir tout le monde?

Sécuriser l'alimentation de la planète

B. HUBERT, O. CLÉMENT (éd.)

La mondialisation côté Sud

Acteurs et territoires

J. Lombard, E. Mesclier, S. Velut (éd.)

ONG et biodiversité

Représenter la nature?

C. Aubertin (éd.)

Le territoire est mort

Vive les territoires!

B. Antheaume, F. Giraut (éd.)

Les virus émergents

J.-F. SALUZZO, L. VIDAL, J.-P. GONZALEZ

Développement durable

Doctrines, pratiques, évaluations

J.-Y. MARTIN (éd.) 


\section{Sociétés \\ environnements, santé}

Sous la direction de Nicole VERNAZZA-LICHT Marc-Éric GRUÉNAIS Daniel BLEY

IRD Éditions INSTITUT DE RECHERCHE POUR LE DÉVELOPPEMENT Collection Objectifs Suds 
Préparation éditoriale, coordination, fabrication Marie-Odile Charvet Richter

Mise en page

Aline Lugand - Gris Souris

Corrections

Yolande Cavallazzi

Maquette de couverture

Maquette intérieure

Aline Lugand - Gris Souris

Cet ouvrage est réalisé en collaboration avec la Société d'écologie humaine (www ecologie-humaine.eu). Il est issu des $18^{\mathrm{e}}$ journées de la SEH intitulées «Milieu de vie et santé. Quelles pratiques interdisciplinaires?» qui ont eu lieu à l'université de Provence (Marseille) du 5 au 7 juillet 2006.

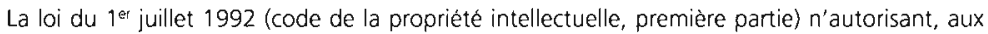
termes des alinéas 2 et 3 de l'article L. 122-5, d'une part, que les " copies ou reproductions strictement réservées à l'usage du copiste et non destinées à une utilisation collective " et, d'autre part, que les analyses et les courtes citations dans le but d'exemple ou d'illustration, " toute representation ou reproduction intégrale ou partielle faite sans le consentement de l'auteur ou de ses ayants droit ou ayants cause, est illicite " (alinéa $1^{\text {er }}$ de l'article L. 122-4).

Cette représentation ou reproduction, par quelque procédé que ce soit, constituerait donc une contrefaçon passible des peines prévues au titre III de la loi précitée.

\section{(C) IRD, 2010}

ISBN : 978-2-7099-1694-3

ISSN : 1958-0975 


\section{Sommaire}

Présentation des auteurs ...................................................... 7

Introduction

Les relations environnement/santé : un champ de réflexion

et d'implication pour les sciences sociales

Nicole VERNAZZA-LICHT, Marc-Éric GRUÉNAIS, Daniel BLEY

Partie 1

L'apport des sciences sociales dans les programmes interdisciplinaires environnement et santé

Mise en œuvre d'un projet interdisciplinaire en matière de prévention des risques sanitaires dans l'estuaire de la Loire:

enjeux et méthodes

Dominique PÉCAUD

La transmission de la maladie de Chagas au Mexique: un exemple méthodologique de collaboration transdisciplinaire

Annie WALTER, Marie-France BOSSENO,

Simone Frédérique BRENIĖRE

La nécessité du recours à l'interdisciplinarité pour étudier le risque de ré-émergence du paludisme en Camargue

Nicolas PONÇON, Céline TOTY, Katrin LANGEWIESCHE,

Alain DERVIEUX, Didier FONTENILLE

Ruralité et asthme en France:

retour d'expérience sur une approche interdisciplinaire 99 Nicole MATHIEU, Marie-Pierre ORYSZCZYN, Wandrille HUCY, Jean MACCARIO, Francine KAUFFMANN

Partie 2

Représentations des risques liés à la pollution

Quand la santé publique redéfinit les enjeux de la pollution atmosphérique en France

Franck BOUTARIC, Pierre LASCOUMES

Attitudes et connaissances des médecins généralistes face aux risques environnementaux

Anne ATTANÉ, Françoise BOUCHAYER, Jean-Christophe MATTEÏ,

Katrin LANGEWIESCHE, MarC-ÉriC GRUENAIS 
Évaluation de la communication en matière de risques liés à l'utilisation des pesticides au Nord-Cameroun

Henry TOURNEUX

Pratiques sociales et risque mercuriel des populations riveraines

du Río Beni en Amazonie bolivienne

Céline TSCHIRHART, Pascal HANDSCHUMACHER, Dominique LAFFLY

Itinéraires des piétons et risque d'exposition à la pollution

en zone urbaine: approche méthodologique

Gilles MAIGNANT, Jérôme DUTOZIA

Partie 3

Comportements humains et maladies liés à l'environnement dans les pays du Sud

L'étude des risques sanitaires liés à l'eau dans l'environnement

urbain: I'exemple de la ville de Chennai, Inde du Sud

Bernard MONDET, Thomas SEYLER, Gérard SALEM,

Jean-Paul GONZALEZ

Risques sanitaires et gestion des eaux usées et des déchets à Kribi

(Cameroun)

René Joly ASSAKO ASSAKO, Carine Alix DJILO TONMEU,

Daniel BLEY

Réflexions sur les obstacles à une prévention efficace

de la maladie du sommeil en Guinée maritime

Jean-Pierre HERVOUËT, Moïse KAGBADOUNO, Mamadou CAMARA

Comportements humains et risque de transmission de la maladie du sommeil en zone périurbaine de Kinshasa (RDC) 315 Pascal GRÉBAUT, Gustave SIMO, Émile Zola MANZAMBI, Jean-Marie BENA, Philémon MANSINSA, Gaëlle OLLIVIER, Gérard CUNY

Un regard d'historien sur la lutte contre le paludisme au Cameroun (1950-1960)

Jean-Paul BADO

La perception du risque palustre chez les femmes

dans deux villes camerounaises: Maroua et Bafoussam

Estelle KOUOKAM MAGNE 


\title{
Présentation des auteurs
}

\section{René Joly Assako Assako}

rjassako@yahoo.fr ;

est géographe, professeur à l'université de Yaoundé-1 (Cameroun) et professeur invité dans plusieurs universités françaises. Prix scientifique de I'Agence universitaire de la francophonie pour jeunes chercheurs en 2007, il est spécialiste de la télédétection et des systèmes d'information géographiques appliqués aux milieux urbains et à l'environnement. Ses récentes recherches portent sur I'interface milieux de vie et santé, champ disciplinaire où il a développé un module d'enseignement et créé, à l'École normale supérieure de Yaoundé, le laboratoire Villes-Environnement-Santé (LaVES).

\begin{abstract}
Anne Attané
Anne.Attane@ird.fr

est anthropologue au sein de I'UMR Inserm/IRD/université de Provence Maladies transmissibles, systèmes de santé, sociétés. Depuis plusieurs années, elle développe une réflexion autour des mutations familiales et des relations de genre en Afrique de l'Ouest et plus particulièrement au Burkina Faso. La dimension matérielle des relations entre les sexes, les âges et les générations est au centre de ses recherches. Elle aborde aujourd'hui ces questions en s'intéressant aux contraintes relationnelles des femmes vivant avec le $\mathrm{VIH}$ au Burkina Faso.
\end{abstract}

\section{Jean-Paul Bado}

.jpaulbado@wanadoo.fr

est historien, chercheur associé à l'université d'Aix-en-Provence, Cemaf Aix, et directeur du groupe de réflexions et d'actions pour la santé en Afrique (Grasa). Spécialistes de l'histoire de la santé, des maladies et des sciences biomédicales en Afrique francophone, ses recherches actuelles portent sur une nouvelle approche de la notion d'espérance de vie à travers l'étude de maladies liées à l'environnement. Son dernier ouvrage paru en 2006, aux éditions Karthala porte sur Les conquêtes de la médecine moderne en Afrique. 


\begin{abstract}
Jean-Marie Bena
jeanmarie_bena@yahoo.fr

est géographe au Bureau d'étude et d'aménagement et de l'urbanisme (BEAU) à Kinshasa. Suite aux conflits en RDC à la fin des années 1990, la majeure partie des documents cadastraux de la ville de Kinshasa avait disparu. Au sein du projet, l'imagerie satellitaire devait permettre au BEAU de reconstituer son cadastre. J.-M. Bena fut chargé de la caractérisation des facteurs environnementaux de la zone d'étude.
\end{abstract}

\title{
Daniel Bley
}

Danielbley@aol.com

est anthropologue, biologiste, directeur de recherches au CNRS et directeur adjoint de l'UMR Espace (équipe Desmid, université de la Méditerranée). II a développé, dans une diversité de lieux et de cultures, des travaux sur le concept de qualité de vie afin d'apprécier la façon dont les hommes s'adaptent à leur milieu de vie. Il anime depuis plusieurs années des recherches interdisciplinaires dans les pays du Sud qui ont pour objectif de comprendre les représentations et les pratiques des populations en matière d'environnement/santé, et s'intéresse en particulier aux maladies transmissibles vectorielles (paludisme au Cameroun, chikungunya à l'île de la Réunion).

\section{Marie-France Bosseno}

mbosseno@ird.fr

ingénieur d'étude (UR Caractérisation et contrôle des populations de vecteurs) à l'IRD, a travaillé sur l'épidémiologie du paludisme au Congo et au Burkina Faso et depuis vingt ans sur la maladie de Chagas ainsi que sur le développement d'outils moléculaires appliqués à la caractérisation des parasites et des vecteurs. Elle est auteur de plusieurs articles et chapitres d'ouvrage dans ces champs de recherche.

\section{Françoise Bouchayer}

bouchayer@univmed.fr

est sociologue, chercheur au centre Norbert-Elias, CNRS-EHESS (Marseille). Ses travaux portent sur les différentes manières d'être un professionnel de santé. Elle a développé dans cette perspective une approche conceptualisée de «la fonction soignante de proximité » à propos des médecins et des paramédicaux de première ligne. Ses recherches sur les questions de santé environnementale interrogent le rapport de ces professionnels aux risques sanitaires, à l'espace local et aux administrations territorialisées. 


\section{Franck Boutaric}

franckboutaric@gmail.com

est docteur en science politique, il enseigne l'économie et la gestion. Nombre de ses publications traitent des enjeux de la pollution atmosphérique; ses recherches actuelles portent sur l'appropriation de la méthode de l'évaluation des risques sanitaires en France et le rôle des instruments d'action publique dans la conduite des politiques environnementales et sanitaires.

\section{Simone Frédérique Brenière}

Frederique.Breniere@ird.fr

est biologiste et directrice de recherche à I'IRD. Ses travaux portent principalement sur l'épidémiologie moléculaire de la maladie de Chagas, en particulier les caractéristiques épidémiologiques des variants génétiques de l'agent infectieux et développent une approche intégrée vecteur-hôte-parasite-environnement, afin de mieux préciser les systèmes épidémiologiques en jeu. Ses travaux se sont développés principalement en Bolivie et au Mexique.

\section{Mamadou Camara}

mamadycamarafr@yahoo.fr

est entomologiste médical, diplômé du CEMV (Centre universitaire de formation en entomologie médicale et vétérinaire) de Bouaké. II est le coordinateur en titre du Programme national de lutte contre la trypanosomiase humaine de Guinée et, depuis 1996, a participé à toutes les recherches menées en collaboration avec l'institut Pierre-Richet de Bouaké et le Cirdes (Centre international de recherche-développement sur l'élevage en zone sub-humide) de Bobo Dioulasso.

\section{Gérard Cuny}

gerard.cuny@ird.fr

est biologiste moléculaire et directeur de l'unité mixte IRD/Cirad Interactions hôte-vecteur-parasite dans les trypanosomoses.

\section{Alain Dervieux}

alderv.desmid@wanadoo.fr

est écologue, plus spécialisé aujourd'hui en écologie humaine et responsable d'un programme de recherches sur la gestion de l'eau dans le delta du Rhône (Eaux et Territoires du MEEDDEM). Ses travaux portent sur la gestion de l'eau en zone humide littorale méditerranéenne, les conséquences pour le territoire, notamment au niveau des milieux naturels ou pseudo-naturels et des paysages. La gestion humaine de l'eau est au centre de son approche (conflits et contradictions). 


\begin{abstract}
Carine Alix Djilo Tonmeu
djilocarine@yahoo.fr

est doctorante en géographie à l'université de Yaoundé-1 (Cameroun). Ses travaux portent sur l'interface environnement/santé. Elle travaille, pour sa thèse, sur l'analyse géographique de la pollution et des risques sanitaires dans deux villes péri-métropolitaines de Yaoundé.
\end{abstract}

\title{
Jérôme Dutozia
}

dutozia@unice.fr

est géographe, doctorant à I'UMR Espace et attaché temporaire d'enseignement et de recherche à l'université de Nice Sophia-Antipolis. II s'intéresse à la vulnérabilité des systèmes territoriaux et mène une recherche doctorale intitulée "Espaces à enjeux et effets de réseaux dans les systèmes de risques " et financée par la région Provence-Alpes-Côte d'Azur.

\section{Didier Fontenille}

Didier.fontenille@ird.fr

est directeur de recherche à l'IRD. Entomologiste médical, il s'intéresse plus particulièrement à la systématique, à la biologie, à la génétique des vecteurs des parasites du paludisme et des virus responsables d'arboviroses. II a essentiellement travaillé sur des problématiques africaines, en particulier à Madagascar, au Sénégal et au Cameroun.

\section{Jean-Paul Gonzalez}

jean-paul.gonzalez@ird.fr

est directeur de recherche à l'IRD. Ses recherches portent sur les conditions et les territoires d'émergence et de diffusion de maladies infectieuses. Il est en détachement au ministère des Affaires étrangères et européennes. Directeur du Centre international de recherches médicales de Franceville au Gabon, il dirige des recherches sur les interactions spatio-temporelles des pathologies au niveau des populations humaines et animales en milieu tropical humide.

\section{Pascal Grébaut}

pascal.grebaut@ird.fr

est assistant ingénieur en biologie à l'IRD depuis 1985; il a travaillé tout au long de sa carrière sur la maladie du sommeil et la lutte contre les glossines. Co-inventeur avec C. Laveissière du piège monoconique "Vavoua », P. Grébaut a activement contribué à la mise en place de dispositifs de lutte antivectorielle à grande échelle au Mali, en Côte-d'Ivoire, au Congo et au Cameroun. Depuis les années 2000, il s'intéresse en particulier à l'évaluation du risque de transmission de la THA. Aujourd'hui, il étudie aussi des aspects plus fondamentaux de la thématique, notamment les protéines excrétées/sécrétées par le trypanosome au cours de la phase invasive chez l'hôte, constituant ainsi autant de cibles thérapeutiques potentielles. 


\section{Marc-Éric Gruénais}

gruenais@ird.fr

est anthropologue, directeur de recherche à I'IRD, membre de I'UMR Sciences économiques et sociales, systèmes de santé, sociétés (Inserm-IRD-université de la Méditerranée). Ses travaux portent notamment sur l'organisation des systèmes de santé locaux, la qualité des soins et les personnels de santé en Afrique. II a également collaboré à plusieurs programmes de recherches sur la prévention et la prise en charge du paludisme.

\section{Pascal Handschumacher}

p.handschumacher@unistra.fr

est géographe de la santé à l'IRD (UMR SE4S). II est actuellement en accueil à la faculté de géographie de l'université de Strasbourg. Ses recherches portent principalement sur les déterminants des inégalités spatiales de santé face aux maladies transmissibles en Afrique de l'Ouest, à Madagascar ou en Bolivie. Ses articles traitent des processus de construction et de fonctionnement des systèmes pathogènes des maladies transmissibles et de leurs espaces d'expression, dans une optique de prévention et d'aménagement orientée vers la santé du territoire.

\section{Jean-Pierre Hervouët}

jphervou@yahoo.fr

est géographe et était directeur de recherche à I'IRD où il initia le grand programme Environnement et Santé avant de créer et diriger le Laboratoire des sciences humaines appliquées à la santé implanté à l'institut Pierre-Richet de Bouaké. Ses travaux le conduisirent, dès 1976, à se placer aux diverses échelles de fonctionnement des phénomènes épidémiologiques déterminées par l'écologie des vecteurs et les interprétations faites des environnements, constructions sociales, par les sociétés humaines.

\section{Wandrille Hucy}

Wandrille.hucy@univ-rouen.fr

est géographe, professeur agrégé à I'IUIFM de l'université de Rouen et chercheur associé à I'UMR Idees et à I'UMR Ladyss. Sa thèse de doctorat Vivre la ville au naturel, une expérimentation sur la ville de Rouen combine de façon originale l'approche spatiale et quantitative et une approche anthropologique du rapport des gens à leurs lieux de vie. Ses travaux portent sur le thème des natures dans la ville et tentent de renouveler les méthodes d'analyse de l'urbain appropriées à des milieux ("durables ») et des dynamiques complexes. 


\begin{abstract}
Moïse Kagbadouno
Moiseake65@yahoo.fr

est entomologiste médical, titulaire du master international d'entomologie médicale et vétérinaire [universités d’Abomey-Calavi (Bénin) et Paul-Valéry (Montpellier)]. II a mené les premières enquêtes pluridisciplinaires sur la maladie du sommeil en zone littorale de Guinée en 1996-97 puis participé à toutes les recherches menées en collaboration avec l'institut Pierre-Richet de Bouaké et le Cirdes de Bobo Dioulasso.
\end{abstract}

\title{
Francine Kauffmann
}

Francine.kauffmann@inserm.fr

est médecin épidémiologiste, spécialiste des maladies respiratoires et directrice de recherches à I'Inserm. Elle dirige l'équipe d'épidémiologie respiratoire et environnementale du centre de recherches Inserm en épidémiologie et santé des populations à Villejuif. Ses travaux portent sur les déterminants tant environnementaux que génétiques des maladies respiratoires chroniques.

\section{Estelle Kouokam Magne}

esthelka@yahoo.com

est anthropologue, chargée de cours à l'université Catholique d'Afrique centrale à Yaoundé et post-doctorante au sein de l'équipe Maladies transmissibles, systèmes de santé et sociétés (MTSS) de I'UMR Inserm-IRD-université de la Méditerranée. Ses travaux portent essentiellement sur la sexualité des adolescents, les relations entre santé et environnement, santé et religions, les interactions entre soignants et soignés, le pluralisme médical au Cameroun.

\section{Dominique Laffly}

dominique.laffly@gmail.com

est géographe, professeur à l'université de Toulouse depuis 2008. II soutient une thèse de géographie à l'université de Franche-Comté en 1995 portant sur l'intégration de la télédétection dans l'analyse quantitative des paysages. Les treize années passées à l'université de Pau et des pays de l'Adour comme maître de conférences seront mises à profit pour diversifier les terrains et les thématiques dans le but de promouvoir des méthodes opérationnelles d'analyse de paysages. Ainsi, Dominique Laffly arpente le territoire et enquête en compagnie de biologistes, de médecins, de physiciens, aussi bien à Madagascar dans le but d'identifier les indicateurs environnementaux de la peste qu'au Svalbard pour quantifier l'impact du changement climatique contemporain sur les écosystèmes polaires. 


\section{Katrin Langewiesche}

katrinlangewiesche@yahoo.fr

est anthropologue et chercheuse associée à l'université de Mayence en Allemagne, à l'Institut d'ethnologie et d'études africaines. Elle s'est spécialisée en anthropologie de l'environnement et du religieux en Europe et en Afrique. Elle coordonne actuellement un programme de recherche concernant «Le secteur sanitaire confessionnel au Burkina Faso. Collaborations et tensions avec le secteur sanitaire public ». Elle a effectué des recherches de terrain de longue durée en France et en Afrique francophone.

\section{Pierre Lascoumes}

pierre.lascoumes@sciences-po.fr

est directeur de recherche au CNRS. II travaille sur les politiques environnementales et les politiques de lutte contre la délinquance financière. Ses travaux sur l'action publique s'attachent à la régulation des enjeux socio-techniques et il développe avec Patrick Le Galès une approche qui met l'accent sur le rôle des instruments et des technologies de gouvernement. Aujourd'hui, dans le cadre du Centre d'études européennes, il mène des travaux sur les origines et la mise en œuvre du règlement Reach (surveillance des produits chimiques) ainsi que sur les évolutions récentes des politiques de développement durable et de ses institutions.

\section{Jean Maccario}

Jean.maccario@inserm.fr

est pharmacien et professeur de biostatistiques à l'université René-Descartes. Ses travaux portent sur les recherches en biostatistiques avec un intérêt particulier pour les aspects de modélisation de données longitudinales.

\section{Gilles Maignant}

maignant@unice.fr

est géographe et mathématicien de formation. Ses travaux portent sur la thématique du développement durable urbain, avec un fort apport méthodologique, notamment en termes de transferts entre disciplines. II est l'auteur de plusieurs articles sur la modélisation de la pollution de l'air en milieu urbain et ses travaux récents portent sur «l'optimisation en géographie ou comment penser la géographie du mieux».

\section{Philèmon Mansinsa}

diabakana@yahoo.fr

est responsable de la lutte antivectorielle au sein du Programme national de lutte contre la THA (PNLTHA) congolais, et supervise l'ensemble des opérations visant à étudier ou éliminer les glossines des foyers de maladie du sommeil en RDC. 


\begin{abstract}
Émile Zola Manzambi
emlzola@yahoo.fr

est technicien entomologiste à l'Institut national de recherche en biologie (INRB, Kinshasa, RDC). II a beaucoup travaillé à l'étude de la transmission palustre à Kinshasa.
\end{abstract}

\title{
Nicole Mathieu
}

mathieu@univ-paris1.fr

est historienne et géographe, directrice de recherche à l'UMR Ladyss (Laboratoire dynamiques sociales et recomposition des espaces), CNRS/université de Paris-IPanthéon-Sorbonne. Ses recherches actuelles portent sur le concept de mode d'habiter mis à l'épreuve de la relation urbain/rural et du développement durable. Depuis le début de sa carrière avec l'enquête pluridisciplinaire de Plozévet jusqu'à aujourd'hui, elle est engagée dans la pratique de l'interdisciplinarité comme méthode pour appréhender les questions et les objets complexes.

\section{Jean-Christophe Matteï}

jcmattei@hotmail.com

est anthropologue de la santé et conseiller en méthodologie et évaluation au sein de l'association AIDES qui œuvre dans le champ de la lutte contre le sida et les hépatites. Ses travaux de recherche à l'EHESS de Marseille (UMR SHADYC) ont porté essentiellement sur l'implication des médecins généralistes dans la prise en charge des personnes vivant avec le $\mathrm{VIH} /$ sida. II a également collaboré à des études de perception des risques environnementaux dans la région de Marseille.

\section{Bernard Mondet}

bernard.mondet@ird.fr

est entomologiste médical à l'IRD jusqu'en 2008, ses recherches ont porté sur l'étude des arboviroses transmises par les moustiques de la zone intertropicale. Il a conduit des travaux en Afrique, en Amérique du Sud, en particulier sur les vecteurs de la fièvre jaune et la compréhension des mécanismes de ré-émergence de la maladie. Affecté en Asie, il s'est intéressé en particulier au virus de la fièvre dengue.

\section{Gaëlle Ollivier}

gaelleo@hotmail.com

est épidémiologiste, ex-attachée culturelle à l'ambassade de France en RDC, elle a contribué à l'évaluation du dispositif de surveillance et de contrôle mis en place par le PNLTHA congolais. 


\section{Marie-Pierre Oryszczyn}

Marie-pierre.picot@inserm.fr

est épidémiologiste, biologiste de formation et ingénieur de recherche à l'Inserm. Ses travaux portent sur les déterminants environnementaux de l'allergie et la mise en œuvre d'outils pour estimer les conditions environnementales à l'aide de questionnaires, de données biologiques ou d'approches écologiques. Elle s'intéresse particulièrement aux effets protecteurs sur la santé des contacts précoces avec les animaux domestiques ou de ferme

\section{Dominique Pécaud}

dominique.pecaud@univ-nantes.fr

est philosophe et sociologue. Enseignant à l'Institut de l'homme et de la technologie (École polytechnique de l'université de Nantes), il est chercheur au CERReV (Centre d'étude et de recherche sur les risques et vulnérabilité) de I'université de Caen et chercheur associé au SEED (Socio-économie, environnement, développement) de l'université de Liège (Belgique). Ses recherches portent sur l'évolution des formes de l'action collective entraînée par les objets ou démarches relevant de la rationalité technique.

\section{Nicolas Ponçon}

nicolas.poncon@agriculture.gouv.fr

est vétérinaire et a réalisé son doctorat au sein de l'UR « Caractérisation et contrôle des populations de vecteurs" de l'IRD. Ses thématiques de recherche ont porté sur les risques de ré-émergence du paludisme en Camargue en abordant différents champs thématiques. II s'est intéressé notamment aux populations de vecteurs potentiels et en particulier à leur dynamique, à leur biologie, à leur compétence vectorielle, et ces éléments ont eté intégrés dans un modèle d'évaluation quantitative du risque de ré-émergence reliant ce risque à l'évolution du milieu sous l'effet des changements globaux.

\section{Gérard Salem}

salem@u-paris10.fr

est professeur des universités (université Paris Ouest-Nanterre) où il dirige le master de géographie de la santé. II est spécialiste desquestions de santé urbaine, ses recherches portant sur la France, l'Afrique et l'Asie du Sud. 


\section{Thomas Seyler}

Thomas.seyler@iss.it

est un épidémiologiste formé à la London School of Hygiene and Tropical Medicine. II est chercheur dans l'unité des maladies transmissibles à l'Instituto Superiore di Sanità (ISS) du Centre national d'épidémiologie, de surveillance et de promotion de la santé à Rome. Ses recherches portent sur l'étude des maladies transmissibles et l'évaluation du risque d'émergence dans des environnements nouveaux (ex.: chikungunya en Italie). Son doctorat en géographie de la santé lui a fait étudier les liens entre le processus d'urbanisation en Inde en regard des facteurs de risque pour la santé, et leur combinaison dans le temps et l'espace.

\section{Gustave Simo}

gustavsca@yahoo.fr

avec un PhD de biologie moléculaire, Gustave Simo s'est engagé dans la recherche sur la THA depuis sa thèse réalisée dans les années 2000 à l'Oceac (Organisation de coordination pour la lutte contre les endémies en Afrique centrale), à Yaoundé (Cameroun). II a en particulier contribué à l'amélioration des outils d'identification moléculaire du pathogène et apporté un éclairage significatif sur la circulation de ce même pathogène dans la faune sauvage dans les foyers de maladie du sommeil au Cameroun. Par ailleurs, il a aussi adaptéla technique d'analyse des repas de sang de glossines par amplification du gène du cytochrome $C$ du sang de l'hôte. Aujourd'hui, G. Simo enseigne à l'université de Dschang (Cameroun).

\section{Céline Toty}

Celine.toty@ird.fr

est ingénieur, spécialisée en entomologie moléculaire. Elle s'intéresse en particulier au complexe d'espèces et à la structuration génétique des populations de vecteurs. Après des recherches conduites à Montpellier, elle est maintenant basée au CRVOI à l'île de la Réunion.

\section{Henry Tourneux}

tourneux@vjf.cnrs.fr

est linguiste, directeur de recherche au CNRS (UMR Inalco, Langage, langues et cultures d'Afrique noire). Son intérêt se porte notamment sur la question de la communication pour le développement dans les domaines de la santé et de l'agriculture. 


\section{Céline Tschirhart}

celinetchi@hotmail.com

est doctorante en géographie à l'université de Strasbourg après avoir été boursière de l'Institut français d'études andines. Ses recherches, menées dans le cadre d'une équipe pluridisciplinaire de l'IRD, portent sur les déterminants géographiques des disparités socio-spatiales de santé. A travers la question de la variabilité de la contamination par le mercure des populations en Amazonie bolivienne, elle s'intéresse tout particulièrement à la production et à la gestion des ressources ainsi qu'aux processus de territorialisation générant sur le long terme une exposition différenciée à ce contaminant.

\section{Nicole Vernazza-Licht}

nvernazza@aol.com

est anthropologue de la santé et chercheur associé à l'UIMR ADES (équipe Société, santé, développement, université de Bordeaux-2). Elle effectue des recherches sur les représentations et la gestion de la maladie et travaille en particulier sur la relation soignant/soigné et sur les itinéraires thérapeutiques. Elle conduit depuis une quinzaine d'années, dans le cadre de projets interdisciplinaires avec des équipes biomédicales, des travaux sur les comportements des populations et des professionnels de santé confrontés à des épidémies comme le sida, le paludisme ou le chikungunya. Elle est l'actuelle présidente de la Société d’écologie humaine (mww.ecologie-humaine.eu)

\section{Annie Walter}

Annie.walter@ird.fr

est ethnologue, chargée de recherche à l'IRD. Ses recherches ont porté sur la gestion traditionnelle des plantes cultivées en Océanie. Elle s'intéresse depuis plusieurs années au rôle des pratiques humaines dans la transmission des maladies à vecteur, en particulier la maladie de Chagas puis la dengue. 



\section{Introduction}

\section{Les relations}

\section{environnement/santé :}

un champ de réflexion

et d'implication

pour les sciences sociales

\section{Nicole VERNAZZA-LICHT}

\section{Marc-Éric GRUÉNAIS}

Daniel BLEY

Le lien entre milieu et pathologie avait déjà été établi par la médecine hippocratique qui accordait une importance majeure aux quatre éléments (terre, eau, feu, air). Le milieu, par exemple le climat, reste associé à de nombreux problèmes de santé ou à des pathologies qui existent souvent à l'état endémique et peuvent prendre des formes épidémiques dans des situations particulières. Ainsi, en France, dans les zones urbaines, on retiendra l'épisode de canicule au cours de l'été 2003 qui a provoqué une surmortalité de personnes âgées, un grand débat médiatique et la démission du directeur général de la Santé de l'époque (ABENHAim, 2003 a). Dans le même esprit, on peut citer le cas du smog à Londres dans l'hiver 1952 qui a vu un pic de décès équivalent à celui de la canicule, mais qui dans ce cas était lié à la conjonction de conditions climatiques particulières avec d'importants niveaux de pollution atmosphérique. Aujourd'hui, ce sont les grippes qui font l'objet d'une surveillance internationale avec les craintes d'épidémies, et les exemples pourraient être multipliés à l'envi... Comme l'écrivait Lucien Abenhaim, «L'environnement a toujours fait partie du 
paradigme de la santé publique, avec l'hôte et le germe ou le poison, c'était même, au début de l'hygiène, le facteur principal explicatif de l'état de santé... La révolution pasteurienne a fait oublier un temps le rôle de l'environnement, en privilégiant celui des germes. Mais les bactéries et les virus sont bien incapables, par eux-mêmes, de produire des épidémies: il faut pour cela qu'ils soient mis en contact, en grand nombre, avec ceux qu'ils vont abattre, souvent par incompatibilité plutôt que par pouvoir pathogène propre. Et c'est l'environnement, dans ces différentes composantes, qui va souvent produire ces expositions " (ABENHAIM, 2003 b).

Mais quelles sont ces composantes? En premier lieu, les composantes "naturelles » qui peuvent permettre à un pathogène particulier, dans certaines circonstances, de s'exprimer tout particulièrement. Le «milieu» auquel il est fait alors référence est principalement le milieu "physique». Max SORRE, dans son article pionnier de 1933, ambitionne de circonscrire ce que l'on pourrait appeler aujourd'hui des «milieux à risque » lorsqu'il propose le concept de « complexe pathogène». Dans une certaine mesure, on pourrait situer le présent ouvrage dans la filiation de cet article de M. Sorre. Bien évidemment, comme on le constatera à la lecture des articles, les paradigmes et les modèles se sont singulièrement complexifiés, et le déterminisme qui transparaissait chez M. Sorre, abandonné. Il convient ici de faire remarquer que, en dépit de son caractère novateur pour l'époque, cet article ne semble guère avoir été à l'origine d'une dynamique de travaux sur le lien environnement/santé.

En fait, au cours du XX' siècle, la santé et l'environnement ont le plus souvent été considérés, à la fois par les décideurs et par les scientifiques, comme des réalités disjointes, et cela malgré l'ancienneté de la reconnaissance du lien entre milieu et pathologie que nous venons de rappeler. Ce n'est que dans les années 1970, qu'émerge une prise de conscience écologique (liée aux dangers de l'urbanisation et de l'industrialisation rapide, à la surpopulation et aux difficultés alimentaires, aux paroxysmes climatiques, et plus récemment aux atteintes à la biodiversité et aux conséquences du réchauffement climatique) qui envisage alors la création de dispositifs prenant en compte les relations entre environnement et développement en général. À l'échelle internationale, c'est la 
conférence de l'ONU tenue à Stockholm en 1972 qui, la première, indique l'urgence de s'intéresser aux questions environnementales. Le rapport Brundtland de 1987 intitulé Notre avenir à tous, qui officialise en quelque sorte l'expression «développement durable», porte donc sur les liens entre environnement et développement, et évoque très largement les dommages liés à la pollution, et l'importance des questions de santé pour le développement. Et c'est seulement en 1995 que l'OMS établit une stratégie mondiale pour la santé et l'environnement; à l'échelle européenne, ce sont successivement la charte de Francfort puis la déclaration d'Helsinki en 1994 qui posent les bases d'une politique commune qui va déboucher sur les plans d'actions nationaux. Mais il aura fallu attendre le début du XXI ${ }^{e}$ siècle, pour que soit reconnu et se concrétise dans des structures officielles le lien environnement/santé. C'est d'ailleurs André Aschieri, chargé de la mission d'étude en France pour la création de l'Agence française de sécurité sanitaire de l'environnement et du travail (Afsset) qui, dans un entretien accordé à la revue Nature, Science, Société notait la difficulté pour les ministères de la Santé et de l'Environnement à collaborer (BLEY, 2001).

À l'évidence, le milieu «physique» a un impact sur la santé des populations. Mais il est tout aussi évident qu'il faut laisser tout déterminisme de côté, et qu'il n'existe pas de relation causale univoque entre l'environnement naturel et la santé des populations. Des historiens de l'Afrique coloniale, par exemple, ont bien montré combien des grands travaux d'aménagement dans les colonies (construction de chemin de fer, aménagements hydrauliques, par exemple) ont pu contribuer très largement à la diffusion de maladies parasitaires (voir par exemple LYONS, 1992). On sait que pour des maladies transmissibles vectorielles comme le paludisme, la dengue ou le chikungunya, le rôle du climat avec la période des pluies est loin d'être le seul facteur, et les activités humaines (aménagements du milieu) ainsi que les perceptions et comportements (inobservance, gestion de l'environnement domestique...) sont aussi à l'origine du développement de ces pathologies. En matière d'arbovirose, selon les spécialistes, ce sont dans $90 \%$ des cas les comportements humains qui sont à l'origine du développement de pathologies. 
Pour appréhender le lien environnement/santé dans toute sa complexité, il est alors indispensable de prendre en compte au moins quatre catégories de facteurs: l'utilisation de l'espace (agriculture, implantations humaines, caractéristiques de l'habitat, environnement naturel et physique); les activités humaines (niveau d'exposition, mobilité, contact avec les zones à risque, types d'activités); les caractéristiques individuelles (connaissance de la maladie, statuts socio-économiques, croyances, perception des risques de santé); les politiques publiques (aménagement des espaces, veille sanitaire et déclenchement des alertes, mise en place de programmes, etc.). Le concept de complexe pathogène tropical (SORRE, 1933) évoqué plus haut est sans doute encore aujourd'hui pertinent pour aborder les relations milieux/santé. Réactualiser ce concept (LE BRAS et MALVY, 2004), c'est aussi envisager de faire travailler ensemble ceux qui étudient l'homme en société (anthropologues, sociologues, historiens, etc.), l'homme en tant que corps biologique (médecins, virologues, etc.), les vecteurs (entomologistes) et les spécialistes du milieu (géographes, écologues...). Il s'agit également par là de donner toute sa place aux recherches interdisciplinaires, associant sciences sociales et sciences de la vie et médicales, à côté de l'apport spécifique des sciences sociales pour l'étude de ces relations. D'une manière générale, cette proposition revient à s'inscrire dans une démarche que nous qualifierons d'écologie humaine, au sens le plus large de l'expression, pour tenter de comprendre comment s'organisent les modalités d'adaptation des hommes dans leurs milieux de vie (DUBOS, 1973).

Cette sensibilité écologique et la démarche interdisciplinaire qu'elle implique souvent sont bien expliquées dans l'ouvrage de Donald HARDESTY (1977) qui décrit comment les scientifiques sont passés par des stades successifs dans l'étude des rapports que les sociétés humaines entretiennent avec leur milieu de vie, et comment les anthropologues ont intégré l'environnement dans leurs recherches. En fait, c'est seulement avec l'émergence de $l^{\prime}$ "écologie scientifique " ${ }^{1}$ que les anthropologues et bien d'autres 
spécialistes de sciences humaines et sociales ont intégré l'idée que les interrelations entre les éléments d'un système permettaient bien souvent d'en comprendre le fonctionnement. Les développements les plus récents s'appuient sur l'utilisation du concept de "système" privilégiant l'interaction constante entre culture, organisations socio-économiques, biologie et environnement. C'est sur l'ensemble de ces bases que nous avons réuni des contributions plus particulièrement de spécialistes de sciences sociales, de sciences médicales et environnementales, tous questionnant la relation entre santé et environnement, au Nord et au Sud, en s'impliquant, pour certains, dans des projets pluridisciplinaires.

La première partie de l'ouvrage s'attache tout particulièrement à relater des expériences de projets pluridisciplinaires, les auteurs de chaque article relevant, pour la plupart des contributions, à la fois des sciences sociales et des sciences médicales. Les quatre articles de cette première partie sont consacrés à des études de cas se rapportant à la France et au Mexique. Au-delà de la diversité des milieux et des problèmes de santé abordés, tous ces articles mettent en avant les négociations, les difficultés, mais aussi les complémentarités dans la conduite de projets interdisciplinaires associant sciences sociales, disciplines médicales et approches écologiques. Ils nous amènent à nous interroger sur l'interdisciplinarité, moins à partir de tentatives de définition ou des aspects positifs ou négatifs de la collaboration entre disciplines en général, mais à partir de pratiques disciplinaires dans le cadre de projets communs.

Sans une approche interdisciplinaire, il n'est pas possible d'atteindre une bonne compréhension des situations étudiées. Par exemple, comment appréhender la prégnance ou la ré-émergence dans un milieu donné des maladies transmissibles vectorielles sans prendre en compte la relation du milieu avec le vecteur d'une part, et les comportements humains d'autre part. C'est ce que montrent Nicolas Ponçon et al., dans le cas de la Camargue, avec une action anthropique, en l'occurrence l'extension de la riziculture, qui pourrait favoriser le risque potentiel de ré-émergence du paludisme. Mais les recherches interdisciplinaires ont aussi des limites. C'est par exemple ce qu'expriment les auteurs sur l'asthme en milieu rural français (Mathieu et al.) qui parlent de «choc des cultures 
scientifiques et des savoir-faire", ou encore ceux qui traitent de la maladie de Chagas (Walter et al.) qui s'interrogent sur « comment construire langage et outils communs» ou «comment mettre en œuvre et interpréter les données". Néanmoins, on peut aussi penser, à l'instar de Dominique Pécaud, à propos des risques sanitaires dans l'estuaire de la Loire, que l'implication de chercheurs en sciences sociales dans les projets, et cela dans une démarche de recherche action, peut éventuellement contribuer à la résolution des conflits, non pas avec la prétention de concilier les points de vue, mais ne serait-ce qu'en mettant en évidence les différences de perception des acteurs (qu'ils soient riverains, administrateurs, experts ou académiques). Aujourd'hui, les sciences sociales et les sciences médicales sont parvenues, peu ou prou, à collaborer dans des projets communs après un long parcours et des cohabitations pas toujours faciles (GruÉnAIS, 1995; PeRREY, DE ThÉ, 2009). Lajout d'un troisième champ disciplinaire relevant des sciences de la nature vient donc encore complexifier la pratique de l'interdisciplinarité.

La deuxième partie rassemble des contributions qui ont en commun d'explorer les attitudes de différents types d'acteurs face à des problèmes de pollution. Elle met l'accent sur l'importance de la perception et de la pratique des acteurs (des décideurs aux bénéficiaires des mesures en passant par les médecins) dans la construction des cadres de référence convoqués pour penser le risque entre santé et environnement. Ainsi, Céline Tschirhart et al., se rapportant aux risques liés à la pollution par le mercure des cours d'eau en Guyane française, nous montrent de manière dramatique combien les usages alimentaires, découlant de pratiques de l'espace et d'activités spécifiques de groupes humains dans une même zone particulière dessinent des risques d'exposition différenciés à un pathogène.

On sait que chaque type d'acteur forge ses propres représentations des risques, et que pour un même contexte, les différentes représentations sont loin de toujours coïncider. On connaît la tendance désormais bien documentée à l'euphémisation des riverains: plus les individus sont confrontés régulièrement et quotidiennement à des risques, plus ils développent des stratégies de minimisation des nuisances. À cet égard, Marie DOUgLas 
(1985) évoquait "l'immunité subjective» qui se développe lorsque les individus sont habitués à la présence dans des sites à risques. À l'inverse, on observe des procédures de dramatisation de la part d'associations défendant les causes des riverains contre une installation à risque, mais aussi de scientifiques afin d'afficher l'urgence sociale des problèmes, et parfois pour légitimer un programme de recherche (FABIANI et THEYS, 1987). Dès lors, il convient non seulement de prendre en compte pratiques et représentations des différents types d'acteurs intervenant sur un site, mais aussi leurs subjectivités qui peuvent conduire à des conflits d'interprétation.

Lorsqu'il s'agit de construire des espaces d'intervention de lutte contre des risques sanitaires, ce sont aussi des trajectoires, et non seulement les territoires, qu'il faut prendre en considération. Ainsi, en ville, selon les trajectoires empruntées, les citadins peuvent être plus ou moins exposés aux nuisances, et les politiques de la ville ont, ou auront, de plus en plus à tenir compte des expositions aux nuisances. Or, les territoires des riverains sont loin de toujours coincider avec ceux que voudraient dessiner les pouvoirs locaux, l'administration, et les chercheurs en écologie et en sciences humaines. S'interroger sur les meilleurs chemins à prendre dans une ville pour être le moins exposé aux nuisances, comme le font Gilles Maignant et Jérôme Dutozia, permet d'introduire une notion appelée à prendre une importance grandissante, surtout si l'on considère la définition élargie de la santé par l'OMS comme état de bien-être, celle de la "qualité de vie" (BLEY, VernazZA-LıCHT, 2006).

Mais qu'offre-t-on aux acteurs, éventuellement, exposés pour les aider à construire leurs perceptions? N'impute-t-on pas trop rapidement aux profanes une part de responsabilité de leur «mauvaise santé » lorsque l'on évoque la nécessité de les éduquer pour qu'ils «changent de comportement $»^{2}$ ? La communication, et partant l'information, est à l'évidence un aspect essentiel de la 
prévention des risques. Il apparaît ainsi que les «communicateurs" en santé ne s'interrogent pas suffisamment sur la réception des messages qu'ils diffusent; dès lors, rien d'étonnant à constater, comme en témoigne l'article de Henry Tourneux sur les mises en garde pictographiques prévenant des dangers de l'utilisation et de la manipulation d'insecticides, que les messages élaborés sont parfois incompréhensibles par les bénéficiaires. Les acteurs les plus patentés, en relation directe avec les populations, sont-ils toujours susceptibles de répondre aux inquiétudes et aux demandes d'information sur les risques environnementaux? Si l'on prend le cas du sud de la France, il faut bien avouer que les premiers «techniciens" interpellés par la population et donc susceptibles de devoir donner une réponse informée, à savoir les médecins généralistes, n'ont qu'un savoir très limité sur ces questions, et leurs connaissances (et leurs appréhensions) ne sont pas toujours très éloignées de celles de leurs patients; ils disposent il est vrai de relativement peu de possibilités de s'informer dans leur pratique courante (cf. A. Attané et al.). Pourtant, ici ou là, on entend évoquer la nécessité de formation spécifique en «santé environnementale» destinée aux praticiens, avec quelques expériences encore très ponctuelles, et on confectionne des manuels ${ }^{3}$. Mais le champ de la santé environnementale est peuplé d'incertitudes, et donc de controverses; les relations causales simples sont difficiles, voire impossibles à établir, et les paradigmes de pensée des experts, dont des éléments sont repérables dans la succession des plans, évoluent rapidement (cf. la contribution de F. Boutaric et P. Lascoumes). Des questions épistémologiques semblent alors toujours devoir être accolées à toute réflexion sur les liens santé/environnement, jusques et y compris dans l'action et dans la décision, et l'apport de toutes les disciplines de sciences sociales s'avère utile et nécessaire.

La troisième partie réunit exclusivement des contributions ayant trait à des problèmes de santé plus spécifiques au Sud (risques hydriques, trypanosomiase humaine, paludisme). Les mises en

$3 \mathrm{Cf}$., par exemple, Les maladies de l'environnement. Un défi pour les professionnels de santé, Embourg (Belgique), Marco Pietteur éditeur (Coll. Résurgence - Médecine et environnement), 2004, ou le gros manuel de M. Gérin et al. (éd.), Environnement et santé publique. Fondements et pratiques, Canada, Editions TEC \& DOC, Edisem, 2003. 
parallèle de situation au Nord et au Sud, telles que nous les avons envisagées dans les deux premières parties, permettent à l'évidence de montrer que des problématiques analogues trouvent toute leur pertinence dans les deux contextes et qu'il convient de ne pas trop singulariser les situations du Sud. Mais cette mise en parallèle a des limites. Les différences d'équipement, la persistance de maladies parasitaires - même si des risques de ré-émergence de pathologies à vecteur sont envisagés au Nord - dessinent des situations sans commune mesure entre les deux contextes. Face au sous-équipement, au manque de réactivité des administrations et services publics, c'est sans doute dans les contextes du Sud que le poids relatif du comportement des acteurs, et en particulier des populations, apparaît comme le plus important pour développer des systèmes explicatifs suffisamment « robustes". Ainsi, l'analyse de Pascal Grébaut et al. sur les risques de transmission de la maladie du sommeil à Kinshasa illustre combien une analyse aussi précise soit-elle du biotope des glossines reste incomplète pour envisager le lien environnement/santé, en l'absence d'une analyse des pratiques sociales et agricoles liées à l'occupation de l'espace. Comme en réponse à cet article, Bernard Mondet et al., à partir de l'exemple de la ville de Chennai en Inde du Sud, montrent combien l'importance des risques d'exposition des groupes les plus démunis aux maladies à transmission vectorielle est liée à une mauvaise gestion de l'eau.

À l'évidence, ce qu'il est convenu d'appeler les «populations » ne sont pas seules en cause, notamment en milieu urbain. En Inde, comme au Congo ou encore au Cameroun, la ville est le lieu par excellence où se développent, dans des espaces relativement restreints, des configurations souvent conflictuelles nées de construction, de pratiques et de représentations d'espace spécifiques à différents types d'acteurs. La gestion des déchets, en milieu urbain, tourne vite à la confrontation (politique) entre municipalités et résidents (Bouju, 2009). Dans quelle mesure, comme le font remarquer René Joly Assako Assako et al. à propos de la gestion des ordures dans une ville secondaire au Cameroun, la constitution de comités d'usagers à la fois contre-pouvoirs et lieux privilégiés de sensibilisation (voire d'éducation) des populations serait envisageable pour réconcilier, ou du moins pacifier, les relations entre les parties. En fait, c'est à une véritable 
administration du territoire, ou des territoires devrait-on plutôt dire, au sens le plus noble du terme "administration", vers laquelle il conviendrait de tendre pour prétendre mieux maitriser les liens santé/environnement. Face à un même risque, à l'évidence, tous les espaces, et donc toutes les populations qui les pratiquent, ne sont pas également exposés. Le défi des recensements et des dépistages systématiquement organisés, par exemple pour la maladie du sommeil en Guinée maritime, dans le cadre de programmes verticaux respectant les cartes sanitaires, constituerait un moyen de contrôle de la maladie. Ici, l'apport des sciences sociales, et en particulier de la géographie, pourrait (devrait) être important, notamment pour la production de connaissances pour l'action, notamment pour circonscrire et hiérarchiser les espaces à risques (cf. J.-P. Hervouët et al.). L'enjeu des sciences sociales est alors de s'inscrire dans un calendrier opérationnel dans le cadre de politiques de santé publique.

Cependant - et le propos de Jean-Pierre Hervouët et al. le rappelle - il est important de prendre en compte la dimension historique. Depuis longtemps, le législateur, et pour l'Afrique en l'occurrence l'administration coloniale, s'est préoccupé de prendre des mesures pour réglementer l'hygiène, définir des zones d'intervention, et établir de véritables cartes d'intervention pour lutter contre les vecteurs. C'est ce qu'indique aussi Jean-Paul Bado lorsqu'il évoque la lutte contre le paludisme au Cameroun avant les Indépendances. Depuis la période coloniale jusqu'à aujourd'hui, pour le paludisme, notamment, mais aussi pour de nombreuses autres pathologies, des initiatives sont prises successivement, procèdent par essai et arrêt, la directive d'aujourd'hui venant parfois remettre en cause totalement celle d'hier. Et là encore, on a souvent beau jeu d'incriminer les populations. Pourtant, les populations se souviennent, comme le montre l'article d'Estelle Kouokam Magne dont les interlocuteurs camerounais rappellent le «bon vieux temps » où il n'y avait plus de moustiques grâce aux aspersions massives d'insecticides à l'époque coloniale et dans les premières années qui ont suivi les indépendances. Les populations ne sont pas a priori ignares mais disposent d'un savoir spécifique qui s'est aussi constitué à partir d'accumulation de messages et d'informations provenant de bribes de savoirs techniques, d'expériences passées, de conceptions populaires. Sans doute, laisse-t-on trop souvent 
les populations faire la synthèse elles-mêmes sans s'interroger suffisamment sur la compréhension réelle des messages de prévention diffusés qui, d'un programme à l'autre, peuvent parfois être contradictoires.

Peut-être y a-t-il plusieurs enseignements à tirer des travaux, des thèmes, des disciplines, des contextes très différents dont il est question dans cet ouvrage. Il est évidemment de toute première importance que les sciences sociales participent pleinement à des programmes pluridisciplinaires, avec les difficultés inhérentes à toute collaboration entre disciplines différentes, comme le montrent les textes de la première partie. Rappelons néanmoins que toute collaboration interdisciplinaire peut s'avérer délicate à négocier, y compris entre disciplines relevant d'un même champ: la collaboration entre démographes et anthropologues (GRUÉNAIS et al., 1985), par exemple, ou encore, entre biologistes, virologistes et immunologistes, n'est pas nécessairement plus facile qu'entre médecins et anthropologues. Par ailleurs, il est tout aussi indéniable que les sciences sociales peuvent avoir un apport spécifique, autonome, pour aider à comprendre, mais aussi pour aider à l'action, avec ce que cela requiert d'ajustement entre l'agenda du chercheur en sciences sociales et celui de l'acteur de santé publique, au Nord comme au Sud. Mais là aussi, il convient de ne pas trop singulariser la situation des sciences sociales qui s'impliquent dans la problématique santé/environnement: toute expertise scientifique, quel que soit son domaine (des sciences les plus «dures» aux sciences les plus «molles»), a son agenda propre qu'il n'est pas toujours aisé de faire coincider avec l'agenda de l'acteur. Le corollaire de la spécificité, voire de la nécessité de l'autonomie de l'apport des sciences sociales dans les travaux sur les liens santé/environnement est la production de travaux disciplinairement très robustes.

Un autre enseignement qu'il convient de tirer de tous les articles réunis dans cet ouvrage est, faut-il le rappeler, que les sciences sociales ne sont pas un tout indifférencié, mais sont constituées d'une multitude de disciplines, qui ont leurs hypothèses, leurs méthodologies, leurs fondements théoriques spécifiques, et que toutes sont bonnes pour penser la relation santé/environnement. À cet égard, sont représentées dans cet ouvrage, entre autres, la 
géographie, l'anthropologie, la sociologie, la politologie, la linguistique, l'histoire. Puisse alors cette entreprise être aussi une invitation adressée à d'autres sciences sociales à s'intéresser à cette problématique. Ainsi, on peut aisément prévoir que les pathologies liées aux pollutions risquent de peser de plus en plus à l'avenir sur les comptes nationaux des États qui peuvent faire bénéficier leurs citoyens d'une couverture sociale, et on aura sans doute à se demander quels seront à terme le nombre de DALYs (Disability Adjusted Life Years) gagnés ou perdus en fonction des mesures prises pour lutter contre le paludisme ou les pollutions et quel en sera le coût? La santé, constituée désormais en bien public mondial, peut aussi évidemment donner lieu à des revendications et à des actions en justice, et les spécialistes du droit ont sans doute ici de beaux chantiers en perspective lorsqu'il s'agit d'établir des responsabilités quant à l'effet néfaste pour la santé de certaines directives. Nous devons donc faire le constat qu'un domaine aussi complexe et spécifique, mais aussi en plein renouveau, n'a pas encore attiré toutes les compétences souhaitables.

Cet ouvrage a pour ambition moins de croiser les regards que de multiplier les angles d'approche sur un objet qui reste encore largement à partager entre sciences sociales, sciences médicales et sciences environnementales en tentant, autant que faire se peut, de désingulariser sinon les contextes, du moins les problématiques. À partir d'exemples empiriques précis, il tente surtout de souligner tout l'intérêt de l'apport des sciences sociales dès lors qu'il s'agit d'envisager les relations entre un pathogène et l'homme. Chacune des approches disciplinaires représentées dans cet ouvrage s'attache ainsi à éclairer les différentes facettes du risque environnemental, depuis le comportement des individus jusqu'aux politiques nationales, en passant par la réponse des systèmes de santé.

\section{Références bibliographiques}

Abenhaim L., 2003 a - Canicules. La santé publique en question. Paris, Fayard.

AbENHAim L., 2003 b - Préface à M. Gérin et al., Environnement et santé publique. Fondements et pratiques. Canada, Éditions TEC \& DOC, Edisem. 
BLEY D., 2001 - Santé et environnement: abolir le fossé. Entretien avec André Aschieri, NSS, vol. 9, n²: 51-55.

Bley D., VernazZA-Licht N., 2006 - "Villes et qualité de vie». In Dorier-Apprill E. (éd.): Ville et Environnement, Paris, édition Sedes, coll. Diem : 109-116.

BOUJU J., 2009 - « Urban dwellers, politicians and dirt: an anthropology of everyday governance in Bobo-Dioulasso (Burkina Faso) ». In Blundo G., Le Meur P.Y. (eds.) : The governance of daily life in Africa, Leiden, E.J. Brill: 143-170.

DOUGLAS M., 1985 - Risk acceptability according to social sciences. New York, Sage Foundation.

DuBos R., 1973 - L'homme et l'adaptation au milieu. Paris, Payot.

FABIANi J.-L., Theys J. (éd.), 1987 - La société vulnérable. Évaluer et maîtriser les risques. Paris, Presses de l'École normale supérieure.

GRUÉNAIS M.-E., 1995 - «Anthropologie médicale appliquée: connaissances, attitudes, croyances, pratique». In Baré J.-E. (éd.) : Les applications de l'anthropologie, Paris, Karthala: 179-220.

Gruénais M.-E., Lacombe B., Boungou G., Guillaume A., 1985 - Une enquête à l'orée de la pluridisciplinarité. Stateco, 43: 5-29.

HARdesty D. L., 1977 - Ecological Anthropology. New-York, John Wiley.

Le Bras M., Malvy D., 2004 - Le complexe pathogène tropical: Regard nouveau sur un concept ancien. Médecine tropicale, 64 (6): 613-618.

Lyons M., 1992 - The Colonial Disease. Londres, Cambrigde University Press.

Perrey C., De THÉ G., 2009 - Le souple et le dur. Les sciences humaines au secours des sciences biomédicales. Paris, CNRS Éditions.

ROBIN J., 1991 - «Les quatre approches de l'écologie». In: Mieux penser l'écologie pour maitriser la production de l'environnement, Transversales, doc. 2: 21-37.

SORre M., 1933 - Complexes pathogènes et géographie médicale. Annales de Géographie, 42: 1-18. 

Partie 1

L'apport

des sciences sociales

dans les programmes

interdisciplinaires

environnement

et santé 



\section{Mise en œuvre d'un projet interdisciplinaire en matière de prévention des risques sanitaires dans l'estuaire de la Loire: enjeux et méthodes}

\section{Dominique PÉCAUD}

Ce texte se donne pour objet d'éclairer du point de vue de la sociologie quelques présupposés sur lesquels repose un programme de recherche nommé Estuae (Entente des sciences et de techniques utiles à l'aménagement de l'estuaire de la Loire). Ce programme s'inspire d'une double volonté. Il vise à mettre en relation différentes disciplines scientifiques afin de comprendre la nature et l'orientation des interactions existantes entre la "physique" d'un territoire, une anthropologie et une sociologie des populations humaines, et la biologie de quelques espèces vivantes indigènes. Il cherche aussi à peser sur l'action publique, via des recherches actions choisies comme méthode d'intervention et comme pratique de mise en œuvre de politiques de prévention des nuisances et des risques sanitaires liés aux effets de ces interactions. Nous évoquerons ici l'interdisciplinarité comme démarche de recherche visant l'apparition de phénomènes émergents par rapprochement disciplinaire. Ce parti pris correspond aux préoccupations des acteurs du programme. 
Tableau I.

Objectifs, méthodes, paradigmes du programme de recherche Estuae

\begin{tabular}{|c|c|c|}
\hline Objectifs du programme & Méthodes & Paradigmes \\
\hline $\begin{array}{l}1 \text { - Connaissance } \\
\text { des populations animales } \\
\text { et des milieux physiques }\end{array}$ & $\begin{array}{l}\text { Inventaire } \\
\text { des données } \\
\text { existantes, } \\
\text { histoire de } \\
\text { leur constitution } \\
\text { et de leur usage }\end{array}$ & $\begin{array}{l}\text { Interdisciplinarité } \\
\text { Émergence }\end{array}$ \\
\hline $\begin{array}{l}2 \text { - Connaissance } \\
\text { des populations humaines } \\
\text { du point de vue } \\
\text { des représentations } \\
\text { et des pratiques de prévention }\end{array}$ & $\begin{array}{l}\text { Enquêtes } \\
\text { de terrain }\end{array}$ & $\begin{array}{l}\text { Lien entre } \\
\text { représentations } \\
\text { et pratiques } \\
\text { Interactions } \\
\text { entre précautions } \\
\text { et prévention }\end{array}$ \\
\hline $\begin{array}{l}3 \text { - Développement } \\
\text { de politiques de prévention } \\
\text { des risques sanitaires }\end{array}$ & Délibération & $\begin{array}{l}\text { Conduite raisonnée } \\
\text { du changement } \\
\text { des pratiques sociales }\end{array}$ \\
\hline
\end{tabular}

Estuae vise l'atteinte de trois grands objectifs. Le premier porte sur la connaissance et l'évaluation des nuisances et des risques sanitaires dus à la présence de moustiques dans l'estuaire de la Loire: Aedes caspius, qui supporte aussi bien les sursalures que les milieux plus doux, et Aedes detritus, inféodé aux seuls sols salés (GABINAUD, 1975). Le deuxième concerne la connaissance des représentations (MAUSS, DURKHEIM, 1903) que les humains ont des nuisances et des risques liés à la présence de ces moustiques, ainsi que des pratiques de précaution ou de prévention que ces dernières inspirent. Le troisième part d'un constat: souvent, les politiques de prévention s'appuient mécaniquement sur les connaissances scientifiques disponibles. Estuae veut comprendre le poids des représentations, donc des connaissances, et celui de la délibération publique sur l'évolution des pratiques de prévention. In fine, il s'agit d'évaluer la portée des politiques de prévention à l'aune de la capacité qu'elles auraient à prendre en compte l'ensemble des logiques d'action développées par les acteurs concernés.

Après avoir défini l'interdisciplinarité, nous dégagerons les enjeux paradigmatiques liés au parti pris méthodologique que cette démarche représente. Puis nous nous interrogerons sur l'intérêt 
d'une déconstruction des principales notions utilisées par Estuae. La déconstruction sera définie ici comme compréhension des processus de construction ayant présidé à l'adoption commune de telle ou telle notion (DERRIDA, 1967). Nous essaierons de comprendre en quoi elle invite à explorer des voies de recherche propices au projet. Ensuite, à travers la question du recueil et du traitement des données, nous présenterons la manière dont se pose la question de l'interdisciplinarité et de ses conséquences du point de vue des pratiques de recherche. Nous nous attacherons ensuite à définir les enjeux politiques liés à la démarche envisagée par le programme. Enfin, une discussion portera sur la nature de l'activité scientifique du point de vue du paradigme de la causalité sociale. En effet, l'activité sociale de la science en matière de prévention des risques peut conduire à une réflexion sur l'articulation entre connaissances, préconisations et changement des pratiques sociales.

\section{La question de l'interdisciplinarité}

Pluridisciplinarité, multidisciplinarité, interdisciplinarité ou transdisciplinarité? L'usage indifférencié de ces termes atténue leurs particularités. Pourtant de nombreux travaux cherchent à les distinguer. Ainsi, la pluridisciplinarité recouvrirait l'usage de disciplines distinctes mais convergentes afin de résoudre un problème. Par distinction ou complémentarité, l'interdisciplinarité évoquera plutôt une méthode cherchant à construire une représentation commune en confrontant les représentations de chaque discipline, affichant la volonté d'une très forte intégration au sein d'une démarche commune (BRUN, 2003).

Dans le cas de la transdisciplinarité, une dynamique intégrative classerait les différentes approches recouvertes par la pluridisciplinarité et par l'interdisciplinarité. L'abandon des canons disciplinaires, voire interdisciplinaires, au profit d'une visée transdisciplinaire aurait également pour conséquence de prendre en compte le rôle de la subjectivité et de la transition intersubjective dans l'élaboration 
des connaissances. Serait ainsi remise en cause la légitimité des séparations héritées de la perspective positiviste entre objet et sujet d'une part, intériorité et extériorité, d'autre part (VALADE, 1999). Edgar MORIN (1982) décrit la transdisciplinarité sous les traits de principes unificateurs en œuvre tout au long de l'histoire de la science. Selon lui, une démarche scientifique ne peut être que transdisciplinaire, soit parce qu'elle utilise la mathématisation et la formalisation indispensables à sa structuration, soit parce qu'elle s'inscrit au sein de postures générales comme l'empirisme, le positivisme ou le pragmatisme qui transcendent la spécificité de l'objet auquel s'intéresse la science. E. Morin considère la transdisciplinarité comme un paradigme qui certes (permet) de distinguer, séparer, opposer, donc disjoindre relativement (les) domaines scientifiques, mais qui (peut) le(s) faire communiquer sans opérer la réduction.

Des connaissances dites émergentes peuvent voir le jour par simple rapprochement de disciplines constituées. Considerons les trois grands domaines: physique, biologie, anthropologie. Comment les faire communiquer? Premier mouvement: il faut enraciner la sphère anthroposociale dans la sphère biologique, car il n'est pas sans probleme que nous soyons à la fois des êtres vivants, des animaux sexués, des vertébrés, des mammifères, des primates. De même il faut enraciner la sphère vivante dans la physis, car l'organisation vivante est originale par rapport à toute organisation physicochimique, c'est une organisation physicochimique, issue du monde physique et en dépendant. Mais opérer enracinement n'est pas opérer réduction: il ne s'agit nullement de réduire l'humain à des interactions physicochimiques, il s'agit de reconnaitre les niveaux d'émergence (MORIN, 1982). Cette proposition trouve son illustration dans l'hypothèse (LALAND, COOLEN, 2004) de "niches écologiques" dont l'évolution serait le résultat d'effets conjoints du métabolisme, des activités et des choix opérés par des êtres vivants. Pour les populations humaines, l'évolution de ces niches procéderait soit d'une "sélection culturelle" comprise comme réaction à une modification conscientisée de l'environnement, soit d'une «sélection naturelle», conséquence d'une modification du même environnement, mais pour laquelle les réponses culturelles se seraient avérées insatisfaisantes. 
Chaque discipline peut apparaître comme élément d'un système interactionnel, les liens entre les différents éléments constituant un domaine de connaissances ou de pratiques émergentes dont les propriétés demeurent irréductibles à chacun des éléments. Cette visée "systémique» (MORIN, 1990) permettrait de dépasser l'obstacle de la «réduction » propre à toute approche disciplinaire, sans que soient remises en cause les garanties scientifiques que cette réduction offre par ailleurs.

\section{Une déconstruction qui s'impose au programme scientifique}

La formulation initiale du programme Estuae utilise des notions comme «territoire» ou "prévention». Ce vocabulaire commun, au sens d'un vocabulaire significativement et socialement disponible, doit être considéré comme l'une des manifestations d'univers sociaux auxquels Estuae se rattache. Mais ces notions, homogènes en apparence, correspondent à des manières de penser hétérogènes et disjointes pour ceux qui les utilisent. Par exemple, ce qui pour l'un définit un territoire spécifique, l'autre n'y voit qu'un espace indifférencié. De même, les manières de minimiser des nuisances, d'évaluer un risque ou de se prémunir d'un danger pourront être différentes selon les personnes concernées. Au cours des échanges qu'ils auront, les différents interlocuteurs useront de ces notions sans obligatoirement en avoir une définition commune, ou sans même vérifier si cette définition est possible. La dynamique des débats se nourrit de la diversité des points de vue auxquels ces notions font implicitement référence. Elle peut prendre également la forme de conflits d'interprétation (RICCEUR, 1969, 1986).

En qualifiant ces notions de prénotions ou de stéréotypes, la discipline sociologique les présente comme l'expression de manières de penser socialement construites. Celles-là ne se présentent pas obligatoirement comme des obstacles à la connaissance scientifique. Elles peuvent elles-mêmes constituer des objets de connaissances (SPENCER, 1875; PATTE, 2005). La pression habituelle des uns sur 
les autres que représente la vie sociale de groupements familiaux, associatifs ou professionnels alimente des pratiques qui vont de soi pour ceux qui les mettent en œuvre. Elle s'exprime sous forme de représentations aux vertus explicatives admises. Les différents patrimoines culturels ainsi constitués participent grandement à la construction des notions évoquées dans Estuae. Mais la constitution d'une vie sociale considérée selon une dimension établie ici et maintenant, dans l'expérience du dialogue et l'élaboration de jeux d'influence représente également l'un des éléments de cette construction. La valeur patrimoniale de ces notions est interrogée parfois brutalement par la confrontation des opinions.

A contrario, la déconstruction permet d'inventorier les points de vue sous-jacents et de mettre en perspective des conditions logiques et sociales de leur compatibilité. Elle permet d'élaborer des hypothèses à propos de l'existence de ces points de vue, et de clarifier les enjeux politiques de leur confrontation ou de leur articulation. La publication du travail de déconstruction peut donner à leurs auteurs l'occasion de délibérer, et d'élaborer ainsi de nouveaux points de vue individuels ou collectifs.

\section{Les présupposés attachés à la notion de territoire}

Estuae décrit l'estuaire de la Loire comme un territoire comportant plusieurs zones humides sur lesquelles cohabitent des espèces vivantes humaines et non humaines. Ces zones sont les suivantes: la baie de Bourgneuf, les marais de Guérande, le lac de Grand-Lieu et la Grande Brière mottière, le marais de Couëron, le marais de Vue, les zones humides de Donges, le marais de Goulaine. Plusieurs d'entre elles sont reliées par des réseaux hydrauliques artificiels (KerouAnton et al., 2000).

La notion de territoire se présente sous les traits d'une notion polysémique. La signification qui lui est attribuée varie selon les personnes et selon les circonstances langagières et sociales. Par exemple, on parlera de territoire de chasse ou de territoire administratif. Elle varie aussi selon les champs disciplinaires utilisés: biologie, géographie, gestion, sociologie, etc. 
Dès que le programme de recherche s'appuie sur la notion de territoire, il semble nécessaire de considérer l'existence de la polysémie et de l'usage du terme comme objet de recherche à part entière. En effet, la pluralité des territoires exprime l'existence de processus de territorialisation et de reterritorialisation (DELEUZE et GUATTARRI, 1980). La recherche ouvre alors à la compréhension de la manière dont sont organisés et justifiés les rapports de coopération ou les situations de conflits autour de l'objet territoire. Les pratiques sociales usant de la notion de territoire sont justifiées par leurs auteurs à partir de la représentation qu'ils se font de ce dernier et des pratiques auxquelles ils s'adonnent. De ce point de vue, le territoire est leur territoire. Il ne peut être question de considérer le territoire comme simple support alors que le définir en termes de construction territoriale rappelle une dimension fondamentale des sciences sociales (RONCAYOLO, 1990). Il convient de recenser les concepts, la forme des énoncés et les usages discursifs (RiccEUR et al., 1977; RiCcEUR, 1986; MONDADA, 1994, 1995) à partir desquels se déploie la notion. Une démarche critique de déconstruction et de reconstruction permet de découvrir les enjeux de rationalité inhérents aux formes de coopération ou de conflits attachés à ce territoire, considéré alors comme le résultat d'une construction sémantique et sociale traitant des interactions entre objets vivants et objets matériels, éléments humains et éléments non humains, présents et passés, etc.

La distinction entre humain et non-humain construit des catégories culturelles et (ou) normatives fondamentales (DESCOLA et PÁLSSON, 1996 ; DESCOLA, 2005). Pour quelques groupements humains, la cohabitation avec des espèces non humaines sur un même «territoire» pourra être ressentie de manière satisfaisante. Tel sera le cas des «admirateurs de la nature» ayant goût, par exemple, à l'observation des oiseaux (CHAMBOREDON, 1985). Mais cette cohabitation pourra être désignée comme source de nuisances, générant alors la volonté de restreindre, voire d'éradiquer des espèces définies comme nuisibles, par exemple, les moustiques. Ainsi, les membres des groupements concernés définiront la nature des nuisances et en rechercheront les causes. Ils auront également le souci d'en mesurer les effets. Ils pourront constituer ou rassembler un ensemble de connaissances savantes, ou confirmer 
des représentations culturelles organisées en points de vue rationnels qui leur paraîtront socialement signifiantes. Dans tous les cas, ces personnes ou ces groupements chercheront à justifier et à valoriser ces differents points de vue, quels que soient les mesures de prévention adoptées et le contenu de significations offert par ces dernières.

Les représentations culturelles et sociales du territoire sont multiples (LÉVI-STrauss, 1958; Durand, 1960 ; Haudricourt, 1962; GODELIER, 1984). Leur confrontation, via la mise en ouvre de pratiques sociales, constitue l'une des bases d'apparition des modes de coopération et de conflits sociaux (MATHIEU et Jolliver, 1989; Sabatier et Ruf, 1992; Claeys-Mekdade, 2000). Il serait donc illusoire de considérer, notamment du point de vue de l'interdisciplinarité, l'estuaire de la Loire sous l'aspect de sa seule réalité physique. Privilégier cette seule réalité, même en considérant les traces d'activités humaines, voire animales ou végétales, ou même en admettant que cette réalité soit appréhendée collectivement par les humains, ne peut écarter le surgissement d'autres manières de voir. Toute définition du territoire qui n'est pas vue comme représentée et construite s'inscrit dans une illusion positiviste. La définition de frontières naturelles capables d'imposer la réalité d'un territoire relève de modes de pensée historique, administrative, patrimoniale ou culturelle. Elle n'a pas d'autre justification.

Si le territoire renvoie à des représentations distinctes, ces dernières sont parfois articulées. Le territoire sera décrit selon sa morphologie physique à travers la question des hauteurs d'eaux temporaires ou permanentes, de l'intensité du débit de la Loire ou de la vitesse de la remontée de la vague de marée, du degré de salinité régnant dans telle ou telle zone humide. La description morphologique pourra être complétée par une description culturelle qui privilégiera le vécu du territoire. On parlera alors d'une manière de lire le paysage, voire d'une manière de vivre attachée à l'espace estuarien, influencée par le rythme des marées qui inversent le cours du fleuve. L'imagination dont ce vécu se nourrit sera d'autant plus mise en avant qu'elle renverra à des particularités physiques prégnantes, le courant du fleuve, la présence du bouchon vaseux poussé et aspiré par chaque marée, les crues, ou encore à des identités culturelles allant de soi, les riverains, les pêcheurs de la 
Basse-Loire, les chasseurs. Linterprétation de l'espace physique, tout en respectant une taxinomie offerte par des disciplines comme la géologie, l'hydrologie ou la géographie physique, pourra être convoquée pour justifier et renforcer l'appartenance à un territoire entendu comme référence symbolique partagée légitimant la relation culturelle entre l'homme et son environnement. On entendra alors évoquer un «climat breton», voire l'existence d'un « esprit ligérien».

\section{Significations et constructions collectives des modes d'explication et d'orientation de l'action}

L'expression culturelle et sociale des territoires symboliques qui cherchent à rendre compte de l'existence d'un territoire réel passe par la mobilisation de pratiques signifiantes pour tel ou tel groupement humain constitué. Ces pratiques relèvent de productions institutionnelles qui se traduisent par des manifestations d'appartenance à un groupe, à une lignée, l'évocation d'une histoire locale, d'un territoire ou d'un "pays d'origine", l'expression ou la revendication d'une coutume. Elles peuvent aussi se manifester par l'adoption de programmes se voulant rationnellement «structurants" comme l'aménagement d'un espace, la promotion d'un produit de terroir, d'une activité typique, ou encore à travers le surgissement de conflits d'usage. Le constat de représentations partagées d'un "monde vécu» (HABERMAS, 1987 a), comme l'engagement dans des conflits, se réalise à la fois à propos de projets d'aménagement de l'espace estuarien, comme le développement du port autonome de Nantes Saint-Nazaire ou celui de la métropole Nantes Saint-Nazaire. On énumérera l'expression d'intérêts divergents concernant l'avenir des zones humides (RAT, 1989), l'exploitation du sable de la Loire, la gestion de la hauteur des eaux qui divise en permanence les agriculteurs, les chasseurs, les associations de protection de la nature. Ces projets sont aussi prétextes à la construction de solutions qui tiennent compte explicitement ou non du pouvoir attribué à des réglementations et (ou) à des groupes. Le territoire témoigne alors d'une appropriation à la fois économique, idéologique et politique de l'espace par des groupes qui se donnent une représentation particulière d'eux-mêmes, de leur histoire, de leur singularité (DI MÉO, 1998). 
Le réel de l'espace physique surgit à travers une réalité construite de manière culturelle, donc implicite, rationnelle, donc volontaire, ou encore conflictuelle (RAMOgNino, 1992). Plusieurs études (VIGARIÉ, 1993) ont montré que des conflits avaient pour principal objet la gestion de l'eau et ses conséquences. Par exemple, la frontière entre zones humides et sèches, entre eau douce et eau salée permet la réalisation d'activités plus ou moins compatibles entre elles: élevage, culture, urbanisation, tourisme, pêche, patrimoine, économie. La fluctuation marine, la hauteur et la nature des eaux sont tributaires des conditions morphologiques, climatiques ou de l'état d'installations hydrauliques, voire des orientations et des effets d'une politique patrimoniale. Des décisions humaines en matière d'aménagement ou de gestion du territoire, comme le projet d'implanter des lotissements dans des zones adjacentes aux marais salants, génèrent ou apaisent ces conflits.

Un recensement de ces conflits permet d'identifier des personnes ou des groupements de personnes aux statuts, aux rôles et intérêts différents. Les élus ont une vision du territoire qui reste en partie dépendante de la nature de leur mandat local ou régional, voire national et des projets politiques qu'ils poursuivent. Différentes associations développeront les programmes d'actions qui répondent à leurs objectifs. Elles privilégieront une donnée du territoire plutôt qu'une autre. Les industriels ou les agriculteurs, les chambres consulaires chercheront à atteindre leurs objectifs en favorisant le développement de telle ou telle activité. Ils évoqueront, selon les cas, zone de pâturage, bassin d'emploi, espace naturel. Les administrations feront appliquer les différentes réglementations concernant la vie d'un territoire dont la définition correspondra à celle de leur activité ou de leur mandat. Les chercheurs de toutes disciplines travaillant sur les problèmes de l'estuaire, en poursuivant leur propre logique de recherche, participeront, à leur niveau, à la définition de ce territoire. Enfin l'ensemble des habitants adoptera autant de rôles que de pratiques différentes, dessinant ainsi autant de territoires. Qu'ils soient citoyens, consommateurs, acteurs économiques ou institutionnels de la vie locale, ils seront parcourus par différentes représentations du singulier ou pluriel.

Définir la notion de territoire par les représentations qu'en ont les populations humaines doit toutefois être complété par la prise 
en compte des populations non humaines et par des traits physiques, chimiques, zoologiques ou écologiques qui s'imposent. On analysera la répartition, la concentration et les déplacements d'espèces. On parlera de " coins à moustiques", de "zones infestées », d'espèces « invasives» (ROUSSEL, MOUGENOT, 2002). On évoquera le rôle de la salinité des eaux pour distinguer des écosystèmes ou des zones de nidification.

Bien que ces différentes définitions et classifications du territoire soient socialement construites, elles peuvent implicitement faire référence à l'existence d'un territoire réel, pensé comme réel au-delà des représentations que chacun en aurait. Il faut donc distinguer le réel supposé du territoire et un espace que cherchent à décrire des études objectives. Les périmètres des objets de recherche, les modèles utilisés permettent de distinguer des espaces différents, selon que l'on s'intéresse par exemple à une espèce précise, à des déplacements, des saisonnalités, à plusieurs espèces et à leurs interactions.

Figure 1.

Les usages et les intervenants des zones humides (d'après CHOUIN, 1998-1999).

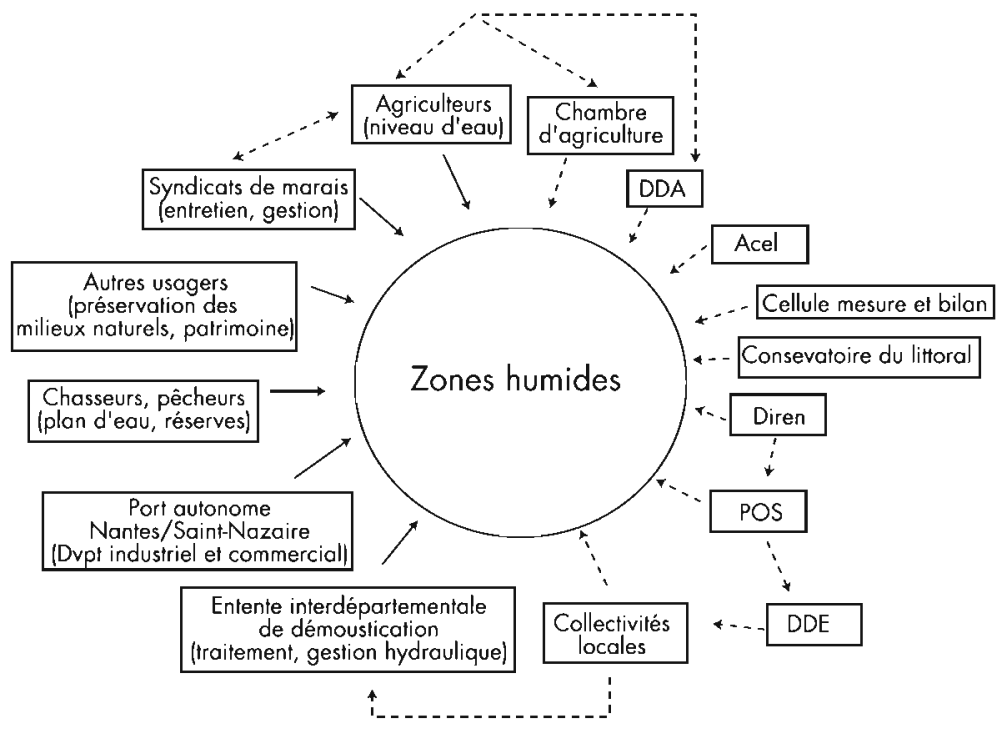


La figure l portant sur les zones humides de l'estuaire de la Loire permet de comprendre la manière dont se construit plus ou moins implicitement cette notion de territoire. Le schéma part de la définition administrative de la notion de zone humide, notion qui garantit implicitement l'homogénéité (administrative) d'un espace et qui discrimine a priori les acteurs sociaux du point de vue des interactions qu'ils entretiennent avec et dans cet espace. À partir de la formalisation de cet espace sont déduits deux types de relations: pratiques de l'espace au sens d'interventions physiques au sein de cet espace, rôles d'acteurs portant sur des interventions de conseil ou de contrôle. Ces relations équivalent à des interactions qui relient des humains définis par leurs rôles et leurs pratiques, des objets que sont, par exemple, les réglementations portant sur les populations humaines mais aussi sur les populations non humaines, des espèces animales. Ainsi, l'activité de la chasse concerne des espèces animales, des chasseurs et des règlements. $\mathrm{Du}$ point de vue des observateurs ou des acteurs, les logiques d'actions qui orientent et justifient les activités humaines peuvent s'opposer explicitement ou implicitement. Elles peuvent également être intériorisées par chacun des acteurs sous forme de conflits de rôle. Lagriculteur peut en même temps être chasseur, creuser des trous d'eau pour attirer le gibier, et subir des nuisances dues à la présence des moustiques.

Selon les cas, le travail de déconstruction pourra prendre comme point de départ le territoire défini selon un corpus administratif de règles (ex.: Convention Ramsar ou programme Natura 2000), selon une profession identifiée (ex.: les pêcheurs de Loire), ou, encore, selon des programmes politiques (ex: l'urbanisation de l'île de Nantes).

\section{Les présupposés attachés aux dangers et aux risques}

La cohabitation entre groupements humains et certaines populations animales (ex: bovins, équidés, canins, félins) peut entraîner des dangers sanitaires objectifs. Ces dangers augmentent mécaniquement lorsque se multiplient les contacts ou lorsque les rapports entre les espèces anthropophiles, les agents pathogènes, les cibles et les milieux évoluent (Marjolet, 1980; Guilloteau et al., 1985). 
Par ailleurs, les représentations des nuisances ou des risques sont le résultat de constructions sociales, historiques et culturelles qui s'élaborent à travers différentes expériences et pratiques et qui se manifestent par l'expression de ces dernières. Ces représentations ne se superposent pas de manière parfaite à des données ayant trait à la connaissance de dangers objectifs. Elles sont le plus souvent interprétations, négations, exagérations ou ignorances de ces dangers. À travers des pratiques qui leur sont attachées, elles peuvent amplifier ou sous-évaluer ce que la connaissance objective des dangers conduirait rationnellement à faire. En effet, les politiques de prévention des risques attribuent généralement un grand intérêt à la transmission de connaissances scientifiques pour modifier les pratiques des acteurs concernés (PÉCAUd, 2005). Selon les cas, les risques seront perçus comme des dangers avérés pour la santé humaine ou décrits comme de simples nuisances. Ils peuvent également être ignorés, sans que ces perceptions aient à voir avec l'objectivité des dangers réels.

\section{Territoire physique, symbolique, dangers et risques}

Les particularités physiques du territoire composent son « réel » au sens où la démarche scientifique chercherait à produire une connaissance objective de celles-là, en dehors de toute influence subjective. De son côté, les représentations de ces mêmes particularités traduisent l'expérience subjective ou sociale qu'ont des groupements humains de ces particularités et de leur agencement signifiant pour soi comme pour les autres. Ces représentations constituent une réalité, considérée par les acteurs sociaux comme réelle. Pourtant, elles s'en distinguent par leur aspect interprétatif spontané qui s'oppose à la réduction volontaire que met en œuvre la démarche scientifique (FrANCK, 1999).

Tableau 2.

Rapports entre données objectives et réalité

\begin{tabular}{|ll|}
\hline Données objectives & Réalité \\
\hline Territoire physique & Territoire «pensé », «agi », « ressenti » \\
\hline Danger & Risque \\
\hline
\end{tabular}


Le rapport entre connaissances objectives et réalité(s) renvoie à la même dynamique sémantique et sociale que celle à laquelle renvoie le rapport entre dangers et risques. Dans une perspective scientifique, la description d'espace physique comme la définition des dangers se veulent objectives. Toute interprétation subjective se doit d'être écartée, grâce notamment à la visée réductionniste à laquelle procède la démarche scientifique. Par contre, la description du territoire pensé, agi, ressenti comme celle des risques renvoie à une série d'interprétations qui constituent l'expression subjective et sociale des normes, de la culture. Par exemple, le danger, défini comme réel objectivable ou comme réel objectivé par l'analyse qui en est faite, que constitue l'inondation avérée a pour condition d'existence minimale l'espace physique pouvant être envahi par l'eau et la quantité d'eau qui s'y déverse (COURTOIs et al., 2002). Mais, du point de vue des définitions données à l'inondation, et surtout des contextes dans lesquels ces définitions sont bâties puis utilisées, il n'existe pas d'équivalence stricte entre l'espace physique de la zone inondable, la définition administrative de cette zone, et la représentation que les populations humaines ont de l'inondation ou de la possibilité de son apparition. Cette représentation disparate se manifeste par des pratiques sociales disjointes, comme la transgression des règles administratives ou le développement de conflits d'intérêts.

Par effet de réduction, le risque induit par l'inondation pourra être calculé à partir d'indicateurs physiques, voire historiques (ex: risque calculé selon la norme de "crue centenaire»). Mais pour atteindre une réalité pensée, il est nécessaire que le risque soit symboliquement signifié grâce à des représentations spécifiques ou des pratiques culturelles plus diffuses (RETIĖRE, 1998). Il doit aussi être socialement construit à travers les formes sociales qui influenceront cette signification et s'en feront l'écho.

Ces différents rapports sont ceux qui séparent, de manière diachronique, la constitution de la science de la fabrication de la norme (HABERMAS, 1987 b). D'un point de vue synchronique, il n'est pas certain que la séparation qu'ils opèrent soit aussi aisée à établir, une réflexion portant à la fois sur l'usage normatif de la science positive, et sur l'empietement de la science positive sur les sciences «historico-herméneutiques » (op. cit.) devant être menée, y compris quand cette séparation se revendique sous son aspect progressiste. 


\section{L'enrichissement des données disponibles}

L'appel à l'interdisciplinarité se justifie par la complexité des objets d'étude et par leur construction à la fois scientifique et normative. De leur côté, les données disponibles pour répondre à l'objectif d'Estuae relèvent d'une production disciplinaire alimentée par le principe de réduction inhérent à chaque discipline concernée.

A partir de ce hiatus apparent, Estuae poursuit une double ambition. Au sein d'une méta-démarche de réflexion et d'action, il cherche à recenser et à rassembler des données scientifiques existantes relevant de disciplines différentes: données des sciences de la nature, comme celles qui concernent l'état des populations de moustiques présentes sur un espace donné, données géophysiques, hydrologiques, etc., mais aussi données disponibles ou à constituer, issues des sciences humaines, comme les données historiques ou les données sociales et économiques portant sur les activités que génèrent l'agriculture, le tourisme, l'urbanisme, l'urbanisation, la santé, ou la gestion hydraulique des eaux de l'estuaire.

Ce premier objectif doit se réaliser par une démarche d'inventaire. Celle-là correspond à une demande relativement neutre proposée sans grandes difficultés aux auteurs et propriétaires des différentes données. En effet, ces données ont été constituées selon une intention définie. De plus, elles ont déjà fait l'objet d'une évaluation scientifique préalable. Leurs auteurs ne peuvent donc pas craindre la remise en cause des données produites. À leurs yeux, la scientificité qui a présidé à leur production joue comme une garantie. Par contre, ils peuvent ne pas être certains des conséquences de la diffusion et de la réception de ces données, dès lors que ces dernières se passent dans un autre cadre que celui où elles ont été produites et validées. Les craintes portent sur la compréhension des données et de leur constitution, et surtout sur l'interprétation qui en sera faite dans un contexte nouveau. En même temps, l'acceptation d'une telle démarche, du fait de la mise en relation des données et de leurs producteurs, amène ces derniers à participer progressivement à une réflexion commune, notamment sur le rôle tenu par les données pour éclairer le programme de prévention porté par la méta-démarche ainsi engagée. 
La mise en relation des données disponibles est censée produire trois effets. Premièrement, elle doit entraîner une mise en relation des auteurs de données. À travers cette mobilisation se met en place l'une des conditions pour constituer une démarche interdisciplinaire. L'effet de "réduction » engendre, de manière quasi mécanique, un effet d'«émergence». Dès que la pertinence des données est confirmée, la démarche peut mener à plusieurs constats: intérêt de mettre en perspective ces données pour élaborer une politique de prévention multifactorielle des nuisances et des risques sanitaires, importance de croiser des données qui ne l'étaient pas jusqu'à présent (par exemple, présence des moustiques et mours des habitants). Deuxièmement, la modélisation des données rassemblées doit favoriser les ambitions prédictives du programme. Troisièmement, la conduite du programme de recherche nécessite la constitution d'un groupe chargé de produire des données complémentaires. La question du statut des auteurs de ces nouvelles données est alors posée, la légitimité de leur expertise également.

Alors que les pratiques de recherche semblent souvent éloignées de ces choix méthodologiques (convergence de données) et politiques (élargissement de la définition des auteurs de données), le programme prévoit la sollicitation des porteurs de la connaissance ordinaire en deux temps différents. D'abord, il offre, à partir d'une enquête auprès des populations, la possibilité d'une prise de parole (indirecte) des populations concernées par la question des nuisances et des risques. L'enquête prévue porte sur les modes de vie et des pratiques sociales, notamment de prévention des populations, liés à la présence de moustiques sur le territoire de l'estuaire. Quand cette prise de parole est scientifiquement valorisée sous forme de données fiables, elle se transforme ipso facto en une prise de position. Dans un deuxième temps, les conditions peuvent être réunies pour éventuellement mettre en œuvre des délibérations éclairées par les connaissances précédemment recensées ou constituées.

Ainsi, à partir d'une démarche qui vise à établir un inventaire des données scientifiques existantes, à en créer d'autres, toutes ces données étant par ailleurs produites dans le cadre de disciplines académiques distinctes, Estuae est enclin à produire au minimum les effets émergents précédemment cités que sont la convergence 
de données et l'élargissement de la notion d'auteurs de données. Il aboutit de facto à une démarche qui dépasse les cadres initiaux ayant présidé à la production des données recensées. Telle est l'ambition de la troisième phase du programme.

Deux phénomènes émergents du programme sont attendus. Premièrement, une reconnaissance croisée de l'intérêt des disciplines académiques engagées est envisagée du simple fait de la valorisation des données produites auprès d'un «collectif» de chercheurs. Deuxièmement, une réflexion sur les interactions possibles entre des données déjà produites et de nouvelles données s'impose pour répondre à l'objectif recherché en matière de prévention des nuisances et des risques. Ces deux phénomènes se rajoutent à d'autres: complexification de l'idée initiale de territoire que s'était donnée chaque discipline dans le cadre de ses propres cadres d'analyse, élargissement du collectif de chercheurs, dès lors qu'il s'agit de transmettre les données aux populations concernées. Toutefois, si ce dernier phénomène est volontairement provoqué au sein de la troisième phase du programme, il n'est pas certain qu'il puisse se réaliser sans difficultés sociales. Il est toutefois important d'envisager de le construire à partir de la compréhension des logiques d'occupation et/ou d'exploitation d'espaces, et de la mise en scène de la justification sociale de ces dernières.

La création d'un "chercheur collectif» (BARBIER, 1996), l'appel à une « intelligence du social» (BERTHELOT, 1990) à laquelle aspire cette troisième phase du programme relève de l'application d'une double perspective méthodologique, celle de la recherche action (GOYETTE et LESSARD-HeberT, 1987; LIU et CreZÉ, 2006) et celle de la sociologie de la traduction (CALlon, 1986). Grâce aux postures qu'elle adopte et aux méthodes qu'elle utilise, la recherche action bouscule les formes habituelles de la division du travail social adoptées par la recherche traditionnelle: remise en cause de la distinction entre chercheurs statutaires et acteurs de terrain, importance attribuée à la délibération comme modes d'élaboration et de transmission des connaissances. Quant à la sociologie de la traduction, elle induit la nécessité d'une collaboration entre chercheurs et acteurs de terrain afin de prendre en compte les intérêts de chacun et favoriser des démarches coopératives éclairées par une connaissance partagée. 


\section{Les auteurs de pratiques peuvent-ils être producteurs de connaissances?}

Estuae affiche l'ambition d'interpeller les populations concernées sur la question des nuisances et des risques sanitaires liés à la présence de moustiques. Deux étapes ont été clairement programmées. La première décrit une étude à caractère sociologique et anthropologique portant sur les usages des habitants du territoire concerné par les risques sanitaires évoqués. Dans la perspective suggérée par S. CHOUIN (1998-1999) ou explorée pour une part par C. Claeys-Mekdade et A. Morales (2000), il s'agit de décrire les modes de vie et les pratiques sociales actuelles des populations concernées directement par la présence de moustiques. La seconde cite une étape qualifiée de délibérative, capable d'éclairer et de mettre en mouvement des processus de décision à la fois valides scientifiquement et socialement. L'articulation d'une politique cohérente de prévention de la santé humaine et d'une politique de développement de territoire semble relever de cette condition.

Différentes démarches prévues par le programme ont pour but de fournir les réponses à plusieurs questions et d'aider ainsi à la construction sociale de propositions de mise en œuvre de solutions, notamment en matière de prévention des risques sanitaires. La première porte sur l'influence de la diffusion de connaissances scientifiques sur l'évolution de pratiques sociales,

Figure 2.

La dynamique de la prévention des risques.

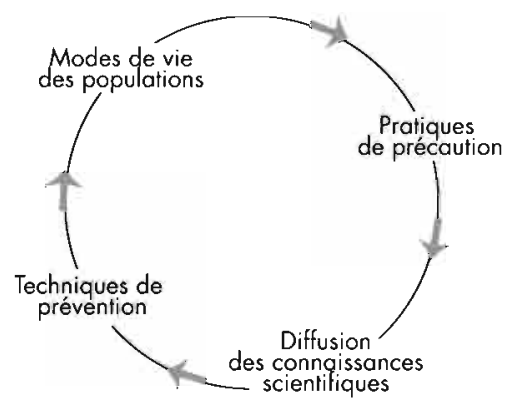


notamment hors de tout système de contrainte réglementaire (LEWIN, 1943 et 1967). La deuxième a trait à l'influence de ces mêmes pratiques sur l'orientation de pratiques d'ingénierie technique axées sur la prévention des nuisances et des risques sanitaires. La prise en compte de ces pratiques peut contribuer à l'émergence d'innovations dans différents domaines: lutte contre les nuisances, réaménagement d'espaces, introduction de nouvelles pratiques économiques, agricoles, touristiques, urbanistiques. La troisième soulève les conditions de mobilisation des équipes de recherche pour constituer et articuler des connaissances multidisciplinaires et contribuer ainsi à résoudre des problèmes de risques sanitaires et d'occupation d'un territoire.

Des incompatibilités objectives ou symboliques d'usage du territoire de l'estuaire existent (COLLONNIER, 1995; KOUADIO-KOUADIO, 1996). Or la capacité individuelle ou collective d'objectiver les conflits d'usage influence-t-elle l'élaboration et l'application d'une politique de prévention des nuisances et des risques? L'avancée actuelle d'Estuae ne permet pas de répondre pour l'instant à cette question. Toutefois, quelle que soit la future réponse, il paraît raisonnable d'affirmer que la réalité des conflits d'usage ne peut que remettre en cause la valeur d'efficacité d'une politique qui ne s'appuierait que sur des seules données objectivées par une démarche privilégiant les aspects physiques du territoire. Laspect délibératif du programme devrait faciliter l'exploration d'autres voies. Mais en affichant cette volonté, Estuae accepte de prendre en compte à la fois la complexité et l'incertitude, mais aussi la possibilité de construire une réalité partagée qui naît de la confrontation des différentes rationalités en œuvre dans l'action collective (MÉRIAUX, 1995; JOBERT, 1995; KuTY, 2001).

Deux niveaux de complexité sont donc proposés. Le premier porte sur la confrontation des données rendant compte de la question des nuisances et des risques sanitaires attachés à l'espace estuarien, le second sur la volonté d'élargir le statut de chercheur à des groupements humains concernés par cette question. Cette dernière option répond elle-même à deux préoccupations: prendre en compte l'avis de populations dans la construction d'une politique de prévention des risques; élargir la base des méthodes et des données de recherche. 


\section{Conclusion}

L'interdisciplinarité, affichée par Estuae, est revendiquée à différentes étapes, dans différents domaines et sous différents aspects. Elle concerne des préoccupations heuristiques touchant à la production de nouvelles connaissances, à la définition de concepts et à l'élaboration de modèles. Son adoption croise des interrogations d'ordre politique concernant le pouvoir attribué aux différents acteurs ou l'orientation des choix axiologiques du programme de recherche. L'intention d'interdisciplinarité soulève également des questions portant sur l'organisation de l'action collective, via le poids des connaissances produites sur la vie sociale, les formes de la division du travail de recherche, les méthodes d'intervention.

\section{La contextualisation comme source de l'exigence interdisciplinaire}

D'un point de vue prédictif, le programme s'est donné pour objectif d'éclairer, voire d'influer les futurs modes de prise de décision dans le domaine de la prévention des nuisances et des risques sanitaires. Au regard de l'intention affichée, il est prévu que cet objectif soit atteint grâce au rapprochement et à la production de données établies dans le cadre de différentes disciplines scientifiques. Il le sera aussi par la mise en cuvre de méthodes capables d'organiser la réception et la diffusion de ces données, voire de générer, à travers des instances délibératives, de nouvelles représentations et de nouvelles pratiques de prévention. Par là, l'ambition d'Estuae recouvre donc aussi bien des préoccupations scientifiques que culturelles ou sociales.

Par la production de connaissances inédites relevant par exemple de la sociologie des risques ou d'une approche anthropologique centrée sur l'usage des "précautions» (PÉCAUD, 2005), le programme cherche à contextualiser les connaissances scientifiques tant du point de vue des conditions de leur production que de celles de leur diffusion. Cette contextualisation renvoie à l'existence de "baquets culturels" définis comme des formes empruntées aux activités prestigieuses ou simplement valorisées (TRIPIER, 1998). 
En effet, jusqu'à présent, les sciences de la nature ont surtout privilégié les connaissances portant sur les interactions existantes entre des espèces animales anthropophiles considérées comme vecteur d'agents pathogènes, les cibles concernées par ces phénomènes et les milieux dans lesquels ces derniers se produisent. Des scénarios de prévention ont pu ainsi en être déduits mécaniquement portant, par exemple, sur la nécessité de démoustiquer telle zone ou de définir telle hauteur d'eau.

Dans le cadre d'Estuae, la contextualisation de ces données porte sur les conditions sociales de leur production et sur les formes de pouvoir dans lesquelles ces données sont valorisées. Elle inaugure une réflexion politique portant à la fois sur les rapports de domination existant entre les différentes disciplines scientifiques concernées et sur la manière dont les connaissances scientifiques sont utilisées pour justifier des décisions. Entre autres, elle permet de préciser les processus de désignation des experts et de comprendre les logiques sociales qui président à la hiérarchisation des acteurs.

\section{Une émergence orientée vers les pratiques de prévention}

Estuae vise au moins deux phénomènes d'émergence exprimés sous forme de deux questions. Quelles nouvelles connaissances est-il possible de produire en rapprochant des connaissances scientifiques existantes? Quels changements de pratiques, quelles solutions de prévention est-il possible de mettre en ceuvre en impliquant les victimes des nuisances ou les cibles des risques dans la compréhension des phénomènes qui les concernent au premier chef?

Répondre à ces deux questions passe par la conception, la mise en œuvre, l'évaluation, la modélisation et la pérennisation des processus de décision concertés à l'échelle d'un espace identifié. La réponse impose également d'approfondir les modèles de la recherche interdisciplinaire et de la recherche-action en facilitant la co-construction d'un processus de recherche et de prise de décision impliquant des chercheurs issus des domaines scientifiques, des chercheurs issus des sciences humaines et sociales, des ingénieurs 
et techniciens, et, à terme, les populations concernées. L'interdisciplinarité affichée dans Estuae prend la forme d'options qui méritent d'être soumises à quelques interrogations.

Tout d'abord, le souhait de recenser les données scientifiques existantes présuppose que ces données soient actuellement dispersées au sein d'ensembles scientifiques homogènes et que leur rapprochement puisse permettre de produire de nouvelles connaissances. Ce présupposé relève implicitement d'une conception de l'activité scientifique vue comme une activité particulière inscrite dans une causalité sociale supposée. En gros, il s'agit de confirmer la proposition avancée par R. K. MERTON (1957) selon laquelle c'est bien l'état social qui pousse à circonscrire et à reconnaître tel ou tel champ d'activité scientifique et non pas le contraire. La nature de cet état social entraîne de fait un type de fractionnement, mais aussi un type de classement des données. Mais ce présupposé peut aussi être analysé sous l'angle d'une sociologie appliquée à la production de connaissances scientifiques telle que la proposent des auteurs comme D. Bloor (1976), B. Barnes et S. Shapin (1979), B. Latour et S. Woolgar (1979), K. Knorr-Cetina (1981), M. CAllon (1986 et 1988), M. Callon et B. LATOUR (1990) pour lesquels les pratiques scientifiques ne sauraient être comprises au regard de seules causes extérieures. Ces auteurs, se situant dans un courant de type externaliste, en appellent à constituer une anthropologie des sciences et des techniques (VINCK, 1995) permettant de dégager les logiques sociales et culturelles propres à l'activité scientifique.

Ensuite, en élargissant la définition des chercheurs à l'ensemble d'une population, c'est donc, par effet de retour, que se trouve mise en question la division du travail social qui préside à l'activité scientifique habituelle. De ce point de vue, la recherche action qui caractérise la troisième partie d'Estuae ne peut que favoriser l'apparition de phénomènes d'émergence. Ces derniers concernent la nature des données rentrant en ligne de compte dans les processus de décision. En matière de prévention, ils visent ainsi les processus de décision eux-mêmes. 


\section{Références bibliographiques}

BARBIER R., 1996 - La recherche action. Paris, Anthropos.

Barnes B., Shapin S. (eds.), 1979 - Sociology of Scientific Knowledge. Berverly Hills, CA, Sage.

Berthelot J.-M., 1990 - L'intelligence du social. Paris, Presses Universitaires de France.

BLOOR D., 1976 - Knowledge and Social Imaginery. Chicago, University Press.

BRUN J.-C., 2003 - Sur les chemins de l'interdisciplinarité ou la documentation et les disciplines scolaires à l'épreuve des nouveaux dispositifs pédagogiques. Paris, Inter $C D I, \mathrm{n}^{\circ} 184$ : 77-87.

CALLON M, 1986 - Éléments pour une sociologie de la traduction: la domestication des coquilles Saint-Jacques et des marins-pêcheurs dans la baie de Saint-Brieuc. L'Année sociologique, vol, 36, Paris, Presses Universitaires de France: 169-208.

CAllon M., (sous la dir.), 1988 - La science et ses réseaux: genèse et circulation des faits scientifiques. Paris, La Découverte, Conseil de l'Europe, Unesco.

Callon M., Latour B. (dir.), 1990 - La science telle qu'elle se fait. Paris, La Découverte.

CHAMBOREDON J.-C., 1985 - «La naturalisation de la campagne: une autre manière de cultiver les simples?"In Cadoret A. (dir.): Protection de la nature, histoire et idéologie: de la nature à l'environnement, Paris, LHarmattan: 138-151.

Chouin S., 1998-1999 - Étude préalable à la lutte contre les moustiques dans l'estuaire de la Loire de Donges-Paimboeuf à Nantes. EID, Conseil général de Loire-Atlantique.

ClaEYS-MEKDADE C., 2000 - Les conflits d'aménagement, rapports à la "nature" et rapports sociaux, la Camargue, un cas révélateur. Thèse de sociologie, université de Provence, Aix-en-Provence.

Claeys-Mekdade C., Morales A., 2000 - Étude d'impact d'un éventuel traitement au B.t.i. sur le territoire du parc naturel régional de Camargue. Rapport scientifique sous la responsabilité de B. Picon, Desmid, Arles.

COLLONNIER A., 1995 - L'estuaire de la Loire, zone naturelle (et) portuaire. Mémoire de DEA, université de Nantes.

Courtois N., Arnal C., Castaing W., Diamandakis M., Giraud F, Houdry P., Mazauric C., Nouguier V., Prastacos P., 2002 - «Anfas: un système d'aide à la planification préventive des inondations par la modélisation hydraulique et la cartographie assistées via Internet. Application à la Loire moyenne . In: Colloque Inondations, Montpellier, France. 
Deleuze G., Guattarri F, 1980 - Mille Plateaux. Paris, Minuit,

Derrida J., 1967 - De la Grammatologie. Paris, Minuit.

DESCOLA P., 2005 - Par-delà nature et culture. Paris, Gallimard.

Descola P., PÁlsson G., 1996 - Nature and Society: Anthropological Perspectives. Londres, Routledge.

Di MÉo G., 1998 - Géographie sociale et territoire. Paris, Nathan.

DurAND G., 1960 - Les structures anthropologiques de l'imaginaire. Paris, Presses Universitaires de France.

FRANCK R., 1999 - La pluralité des disciplines, l'unité du savoir et des connaissances ordinaires. Sociologie et sociétés, vol, XXXI, nº 1 : 129-142.

GABINAUD A., 1975 - Écologie des deux Aedes halophiles du littoral français - Aedes (Ochlerotatus) caspius (Pallas, 1771) Aedes (Ochlerotatus) detritus (Haliday, 1883) (Nematocera-Culicidae). Utilisation de la végétation comme indicateur biotique pour l'établissement d'une carte écologique. Application en dynamique des populations. Thèse de doctorat, université des Sciences et Techniques du Languedoc, centre universitaire de Perpignan.

Godelier M., 1984 - L'idéel et le matériel. Pensée, économies, sociétés. Paris, Fayard.

GOYETTE G., LESSARD-HEBERT M., 1987 - La recherche action: ses fonctions, ses fondements et son instrumentation. Québec, Presses de l'université du Québec.

Guilloteau J., Mas J.-P., Marjolet, 1985 - La lutte physique: un des éléments de la lutte intégrée contre les aédines du littoral atlantique. Bulletin de la Société française de Parasitologie, $\mathrm{n}^{\circ} 1$ : 151-154.

Habermas J., 1987 a - Théorie de l'agir communicationnel. Paris, Fayard.

HABERMAS J., 1987 b - Logique des sciences sociales et autres essais. Paris, Presses Universitaires de France.

Haudricourt A. G., 1962 - Domestication des animaux, culture des plantes et traitement d'autrui. L'Homme II (1) : 40-50.

JOBERT B., 1995 - «Rhétorique politique, controverses scientifiques et construction des normes institutionnelles: esquisse d'un parcours de recherche». In A. Faure (éd.): La construction du sens dans les politiques publiques, débats autour de la notion de référentiel, Paris, L'Harmattan.

Kerouanton J.-L., Lemerle E., Triviere F.X., Vauthier-Vezier A., 2000 - Eau et patrimoine en pays de Retz, le canal maritime de la Basse-Loire et les marais du Tenu. Nantes, Inventaire général (ADAGP)/ADIG.

KNORR-CETINA K., 1981 - The manufacture of knowledge: An essay on the constructivist and contextual model of science. New-York, Pergamon. 
KouAdio-Kouadio Y., 1996 - Protection d'un estuaire: le cas de l'estuaire de la Loire. Revue Juridique NEPTUNUS®@, NEPTUNUS® Law Review@.

KUTY O., 2001 - «L'intervention: système stratégique et communauté de projet sur l'espace public. "In Vrancken D., Kuty O. et al. : La sociologie et l'intervention: enjeux et perspectives, Bruxelles, De Boeck Université: 131-166.

LALAND K., COOLEN I., 2004 - La culture, autre moteur de l'évolution. La Recherche, $\mathrm{n}^{\circ} 377: 52-57$.

LATOUR B., WOOLGAR S., 1979 - Laboratory life: The social construction of scientific facts. Londres, Sage.

LÉvi-Strauss C., 1958 - Anthropologie structurale. Paris, Plon.

LEWIN K., 1943 - Forces Behind Food Habits and Methods of Change. Bulletin of National Resources Council, $\mathrm{n}^{\circ}$ 108: 35-65.

LEWIN K., 1967 - Psychologie dynamique, les relations humaines. Paris, Presses Universitaires de France.

LIU M., CREZE F., 2006 - La recherche-action et les transformations sociales. Paris, L'Harmattan.

MARJOLET M., 1980 - "Moustiques et nuisances en presqu'île guérandaise». In: Marais salants. Contribution à l'étude écologique de la presqu'île guérandaise, Bulletin de la Société des Sciences naturelles de l'ouest de la France, hors-série: 285-288.

Mathieu N., Jollivet M. (dir.), 1989 - Du rural à l'environnement, la question de la nature aujourd'hui. Paris, ARF/L'harmattan.

Mauss M., Durkheim E., 1903 - De quelques formes primitives de classifications. Contribution à l'étude des représentations collectives. Année sociologique, VI: 1-72.

MÉRIAUX O., 1995 - «Référentiel, représentation(s) sociale(s) et idéologie». In Faure A. (éd.): La construction du sens dans les politiques publiques, débats autour de la notion de référentiel, Paris, L'Harmattan.

MerTon R. K., 1957 - Social Theory and Social Culture. New-York, Free Press of Glencoe.

MONDADA L., 1994 - Verbalisation de l'espace et fabrication du savoir: approche linguistique de la construction des objets de discours. Suisse, université de Lausanne.

MONDADA L., 1995 - La construction discursive des objets de savoir dans l'écriture de la science, Réseaux, 71 : 55-77.

MORIN E., 1982 - Science avec conscience. Paris, Fayard.

MORIN E., 1990 - Introduction à la pensée complexe. Paris, Seuil. 
PATTE Y., 2005 - Sur l'utilisation des " catégories indigènes " en sociologie. Recherches européennes en sociologie des medias. Adresse URL: http://www.sociomedia-europe.com/document.php?id=107, Page consultée le: 19/08/2006.

PÉCAUD D., 2005 - Risques et précautions, l'interminable rationalisation du social. Paris, La dispute.

Ramognino N., 1992 - L'observation, un résumé de la réalité. Current Sociology, Vol. 40, $\mathrm{n}^{\circ} 1$ : 55-75.

RAT A., 1989 - Étude des zones humides de la Loire sur sa rive nord de Coueron à Lavau, les modes d'exploitation des zones humides du nord de l'estuaire de la Loire ou les stratégies d'occupation des marais entre Couëron et Lavau. Mémoire de DESS Aménagement, Urbanisme, Environnement et Collectivités locales, APEEL.

Retière J.-N., 1998 - Quand la Loire déborde. Pratiques et représentations de la crue ligérienne, $X I X^{c}-X X^{c}$. Conservatoire des rives de la Loire et région des Pays de la Loire, CENS, MSH Ange Guépin.

RICEUR P., 1969 - Le conflit des interprétations, essais d'herméneutique. Paris, Seuil.

Ricceur P., 1986 - Du texte à l'action. Essais d'herméneutique. T. II, Paris, Esprit/Seuil : 39-73.

RIC@ur P. et al. 1977 - La sémantique de l'action. Paris. CNRS.

RONCAYOLO M., 1990 - La ville et ses territoires. Paris, Gallimard.

ROUSSEl C., MOUGENOr C., 2002 - À qui appartient le ragondin? Espaces et sociétés, $\mathrm{n}^{\circ} 110: 11$.

SABATIER J.-L., RUF T., 1992 - «La gestion sociale de l'eau ». In: La gestion sociale de l'eau, Montpellier, Orstom : 5-8.

SPENCER H., 1875 - Introduction à la science sociale, Paris, Germer Baillière.

TRIPIER P., 1998 - «Théologie ou sciences naturelles à l'origine de la sociologie». In Jacques-Jouvenot D. (dir): L'œil du sociologue, études réunies autour d'Henri Mendras, Besançon, Presses du Centre Unesco de Besançon.

VALADE B., 1999 - Le sujet de l'interdisciplinarité. Sociologie et sociétés, vol. XXXI, $n^{\circ} 1: 11-21$.

VIGARIÉ A., 1993 - État des connaissances et problèmes d'aménagement des zones humides de l'estuaire de la Loire. Cahiers Nantais, n 39: 81100.

VINCK D., 1995 - Sociologie des sciences. Paris, Colin. 


\section{La transmission de la maladie de Chagas au Mexique: un exemple méthodologique de collaboration transdisciplinaire}

\section{Annie WALTER}

Marie-France BOSSENO

\section{Simone-Frédérique BRENIÈRE}

La maladie de Chagas, ou trypanosomiase américaine, est due à un parasite flagellé, Trypanosoma cruzi, transmis à l'homme et à de nombreuses espèces de mammifères par des punaises de la famille des réduvidés. C'est une anthropozoonose complexe dont l'agent pathogène, composé de nombreux clones naturels ${ }^{1}$, peut évoluer d'un hôte à l'autre (dont l'homme) essentiellement par l'intermédiaire des insectes vecteurs. Cette maladie, classée par l'OMS parmi les maladies négligées touche, de 16 à 18 millions de personnes en Amérique latine où la maladie est endémique. Elle pèse d'un lourd tribut (DALYs 667000) $)^{2}$ en termes de santé et entraîne plus de 50000 décès par an. Le nombre de personnes exposées au risque de transmission est estimé à environ 120 millions, la plupart résidant en zone rurale.

1 Le mode de multiplication de Trypanosoma cruzi est essentiellement clonal et il existe une grande variabilité génétique entre les clones

2 DALYs - Disability Adjusted Life Years: the number of healthy years of life lost due to premature death and disability (nombre d'années de vie en bonne santé perdues chaque année en raison d'une mort prématurée ou d'une invalidité). L'indice se calcule sur toute la zone endémique. 
Le parasite, dès sa transmission, envahit les cellules de nombreux tissus dont le cœur et le tube digestif, il s'y multiplie puis passe dans la circulation sanguine se disséminant dans tout l'organisme pour envahir de nouvelles cellules saines. Il provoque une maladie qui évolue en trois phases. La première ou phase aiguë dure de 4 à 8 semaines. Elle passe généralement inaperçue bien que des formes mortelles soient observées chez l'enfant en bas âge. Les symptômes sont généralement peu marqués: seule une réaction inflammatoire localisée au niveau de la peau (chagoma) ou au niveau de l'œeil (signe de Romaña) peut orienter le diagnostic. Par la suite, la désorganisation des défenses immunitaires de l'hôte infecté entraine une fièvre et une asthénie, qui peuvent durer un mois ou deux. Le parasite se multiplie rapidement provoquant une parasitémie sanguine importante mais transitoire qui disparaît en quelques semaines. Durant les années suivantes se déroule une phase latente durant laquelle les défenses immunitaires sont progressivement restaurées, le malade restant porteur du parasite. Elle aussi passe inaperçue. Finalement survient, dans $30 \%$ des cas et souvent des années plus tard, la phase chronique qui se traduit par des lésions cardiaques (arythmie, cardiopathies) et digestives (méga-œesophage, mégacôlon) irréversibles liées à des processus inflammatoires et auto-immuns. La pauvreté des signes cliniques rend difficile un diagnostic précoce si bien que la maladie ne se révèle généralement qu'au stade des lésions irréversibles. Seule l'observation du parasite dans le sang (méthode peu sensible passée la phase aiguë) ou la sérologie mettant en évidence des anticorps spécifiques, présents durant toute la durée de l'infection, peuvent apporter la preuve de l'infection parasitaire.

La transmission de T.cruzi à l'homme se fait principalement à l'occasion du repas sanguin des punaises, durant la nuit, quand la personne dort. La piqûre est lente (environ $15 \mathrm{~min}$ ) et suivie d'une défécation de l'insecte sur la peau du patient. Les parasites qui se trouvent dans ces déjections pénètrent alors dans l'organisme par la lésion que provoque la piqûre. La transmission peut être aussi congénitale, par transfusions sanguines et quelques cas de transmission orale due à l'absorption de boissons contenant accidentellement des broyats d'insectes ont été signalés.

Un concept de base fonde toutes les études épidémiologiques de la maladie de Chagas: il existe deux cycles différents qui se 
déroulent spatialement dans les aires partagées par les différents intervenants et qui peuvent se chevaucher, le cycle zoonotique (parasite, vecteur et mammifères qu'ils soient sauvages ou domestiques) et le cycle chez l'homme également véhiculé par l'insecte vecteur. Leur étude nécessite donc une approche intégrée d'autant plus que les facteurs liés à la transmission vectorielle dépendent fortement de l'environnement et des conditions socioéconomiques.

Le parasite, Trypanosoma cruzi, serait très ancien (YEO et al., 2005) et provoquait à l'origine une zoonose mettant en jeu le seul cycle zoonotique. Progressivement l'homme aurait créé les conditions de sa propre infestation, d'une part en pénétrant dans l'espace initialement partagé par les seuls mammifères et punaises vecteurs, d'autre part en offrant à ceux-ci des conditions d'habitat propices à leur développement. Le processus de contact de plus en plus intime entre l'homme, le vecteur et le parasite n'est pas récent puisque la maladie a été suspectée chez des momies (GUHL et al., 1997; FERrEIRA et al., 2000). Aujourd'hui, Triatoma infestans, le vecteur principal et le plus redoutable de la maladie de Chagas, est «domestiqué » et déroule son cycle biologique dans la maison et ses annexes. Chacune des autres espèces jouit d'un degré variable de «domestication» ou d'adaptation à l'habitat humain dans lequel elle pénètre de façon active, en volant à partir de son habitat sauvage (souvent à la faveur d'un déboisement) ou de façon passive, transportée dans les effets des hommes.

Il n'existe pas de traitement pleinement satisfaisant ni de vaccin contre la maladie de Chagas si bien que la lutte antivectorielle a été mise en place pour lutter contre elle. L'habitat propice au développement des espèces domestiquées, les maisons aux toits végétaux ou aux murs de terre dans lesquels les insectes se nichent facilement, a été la cible des grandes campagnes d'élimination des vecteurs et principalement de T. infestans qui avait fini par se disperser dans tout le continent sud-américain. Ces campagnes se sont fondées sur la pulvérisation d'insecticides dans l'habitat, sur son amélioration et sur l'éducation sanitaire. Elles ont été efficaces et $T$. infestans a disparu de nombreux pays, entraînant une régression notable de la transmission. 
Néanmoins, pour de nombreuses raisons, la maladie de Chagas doit rester une préoccupation majeure des autorités sanitaires des pays du continent américain. En effet, dans les zones traitées de nouveaux profils de transmission vectorielle émergent, dus à la ré-infestation des domiciles par des insectes sauvages locaux ou à des insectes résistants aux insecticides. En dehors des zones traitées, il existe de nombreuses zones de faible endémie qui impliquent bien souvent des vecteurs sauvages plus ou moins adaptés à l'habitat humain. Jusqu'à présent, elles ont peu retenu l'attention des autorités malgré l'impact en santé publique qu'elles représentent. Un faisceau de facteurs variablement intriqués dessine des paradigmes épidémiologiques nouveaux ou ré-émergents. Des facteurs biotiques et abiotiques sont à l'œuvre au niveau des processus de domestication, de résistance aux insecticides, de comportement des insectes. Des facteurs socio-économiques et culturels influent sur la dispersion et sur la domestication des insectes ainsi que sur la façon dont ils sont perçus et tolérés par la population, au sein même de la maison. La lutte contre la trypanosomiase américaine reste une priorité pour de nombreux pays d'Amérique latine et réclame la participation de différentes disciplines pour mieux comprendre l'installation, le déroulement, l'évolution et les transformations des cycles épidémiologiques. Les programmes de recherche et de lutte contre la trypanosomiase américaine, fondés pour la plupart sur des approches biologiques centrées sur le vecteur ou le parasite, font de plus en plus souvent appel à des disciplines issues des sciences sociales (géographie, sociologie ou ethnographie) afin d'analyser le rôle de l'homme dans le système parasitaire. Ces approches multidisciplinaires, nécessaires à l'étude de nouveaux paradigmes épidémiologiques, posent en elles-mêmes de nombreux problèmes méthodologiques qui seront présentés succinctement dans cet article.

\section{La situation mexicaine}

Au Mexique, pas moins de 31 espèces différentes de triatomes ont été décrites dont 18 au moins sont infectées par T.cruzi et neuf d'entre elles auraient actuellement un rôle dans la transmission de la maladie. Toutes ces espèces présentent des populations sauvages qui sont largement réparties sur le territoire. 
Bien que plusieurs de ces espèces soient retrouvées dans les maisons où elles agressent les habitants provoquant ainsi la transmission du parasite, il est difficile d'évaluer leur rôle car il existe peu d'études sur leur écologie, leur distribution géographique et leur comportement dans les milieux anthropisés et sauvages du Mexique. De façon générale, ces systèmes épidémiologiques correspondraient à des endémies faibles mais les enquêtes sérologiques menées au Mexique n'informent pas de façon précise sur la relation entre la dynamique des vecteurs et l'incidence ou la prévalence de l'infection. Dernièrement, des études entomologiques menées dans plusieurs villages de l'Occident mexicain ont montré que le péridomicile, c'est-à-dire l'espace entourant la maison et utilisé par l'homme et les animaux domestiques, était lourdement infesté par les réduves. Les auteurs ont désigné l'infestation péridomiciliaire comme un facteur de risque majeur de la transmission du parasite (MAGAllón GASTÉlum et al., 2006). C'est, de fait, un espace quotidiennement partagé entre le parasite, les populations de vecteurs domiciliées (celles qui se reproduisent à cet endroit) et éventuellement les populations de vecteurs sauvages, les hommes, les animaux domestiques et les animaux sauvages qui y font des incursions, sans oublier les éléments végétaux présents comme le bois mort. Cependant, la transmission proprement dite a lieu à l'intérieur de la maison, l'homme n'étant généralement pas exposé à la piqûre des réduves durant la journée dans le péridomicile.

Les mêmes études entomologiques ont montré que l'infestation de ces péridomiciles variait, entre villages et entre unités d'habitation d'un même village, mais les conséquences sur la transmission restent mal connues. Souvenons-nous que le péridomicile est un espace fortement anthropisé, conçu et organisé par l'homme qui y réside. Il sert de lieu de vie à plusieurs espèces vivantes, vecteurs et hommes compris, si bien que le comportement des unes et des autres influe sur leur contact réciproque, sans que l'on sache avec quelle fréquence et dans quelles conditions. Au Mexique, dans certains villages étudiés, le patio (c'est-à-dire le péridomicile) est généralement un espace fermé, borné sur un côté par la façade arrière de la maison et sur les trois autres par de hauts murs le séparant de ses voisins. Il contient quelques remises, des abris ou auvents, des piles de matériel de construction tel que briques et tuiles, différents objets gardés là en attendant un éventuel usage et, s'il est assez vaste, une seconde maison destinée à loger un 
autre membre de la même famille (photo 1). Chien, chat, volaille et cheval y séjournent; les hommes y bavardent, lavent le linge, cuisinent parfois, coupent du bois, y mènent de nombreuses activités; les rats y profilèrent (Bosseno et al., 2006), au moins deux espèces de triatomes s'y installent. Ailleurs, le péridomicile apparaît comme un espace plus ouvert, clôturé à minima, dans lequel la végétation est plus dense et l'incursion des animaux sauvages sans doute plus fréquente (photo 2 ). En réalité, et même si tous ces patios se ressemblent, chaque famille y imprime sa marque, en le gérant de façon singulière. Dès lors, y a-t-il des facteurs qui favorisent la pénétration et la multiplication des vecteurs dans un péridomicile particulier? Relèvent-ils de la structuration de l'habitat ou d'une gestion particulière du milieu? Une approche conjointe biologique (comportement des vecteurs et caractéristiques du parasite) et ethnographique (comportement des hommes et structuration de l'habitat) est donc indispensable pour pouvoir mieux appréhender les processus d'installation des vecteurs et les risques de contact avec les populations humaines.

Photo 1.

Les péridomiciles fermés et la densité du bâti à Los Guerrero.

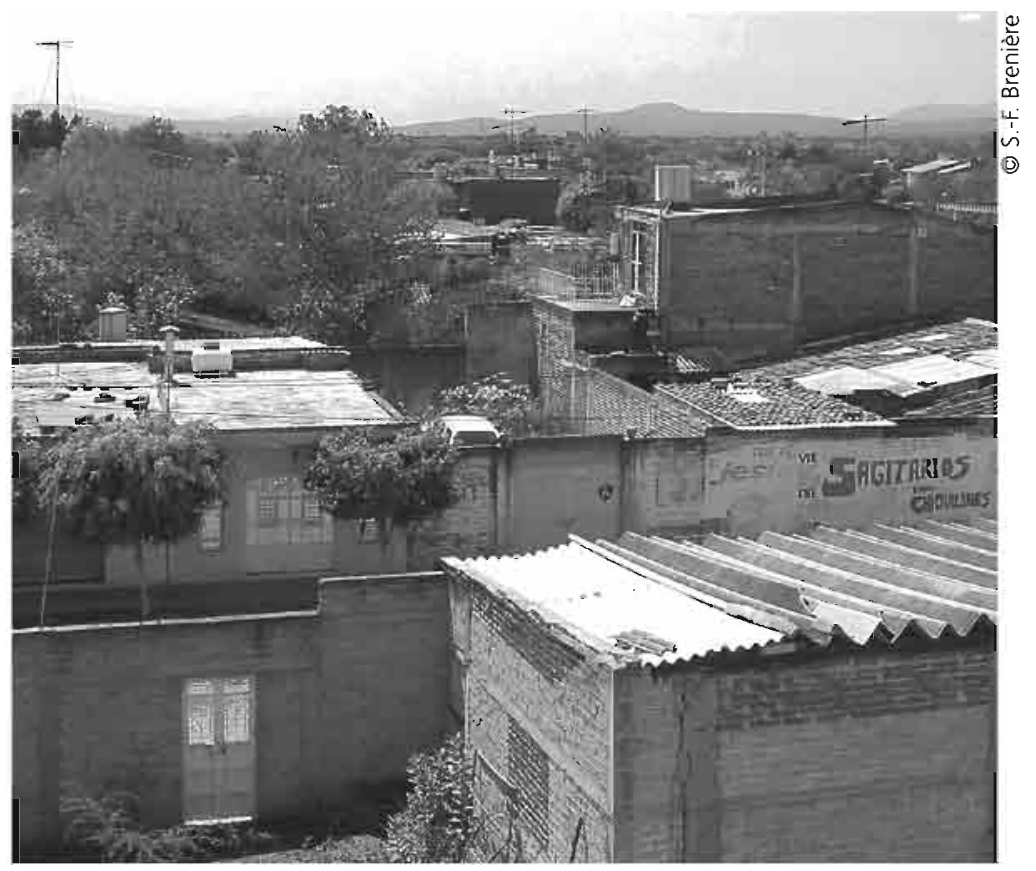


Photo 2.

Les péridomiciles ouverts de Cacalutan,

à la végétation plus dense.

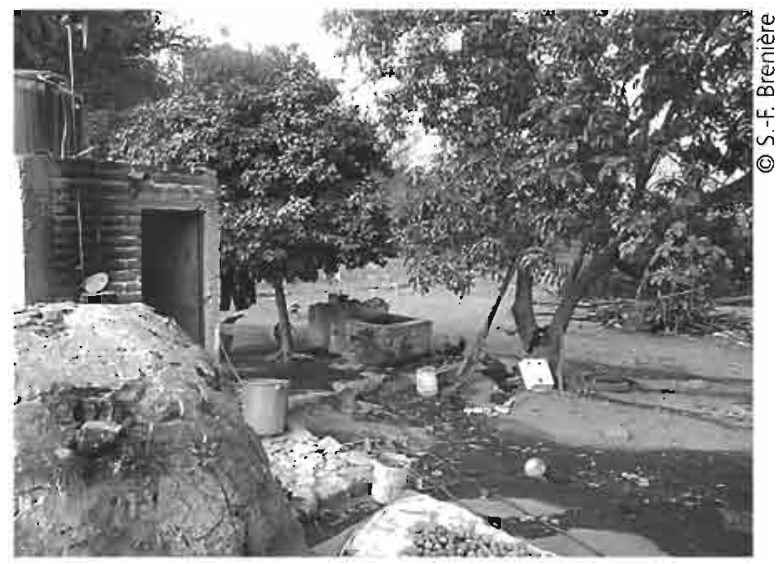

\section{Construction d'un langage et d'outils communs}

Le premier travail des chercheurs issus de disciplines différentes a été de construire un glossaire commun des termes et des concepts qui devaient être utilisés pendant la recherche. Il a pris en compte les définitions des structures visibles (maison, péridomiciles, structures du péridomicile), des espaces (espace du village, espace agro-pastoral, espace sauvage), des personnes (famille, ménages) et des concepts (infestation, représentation, etc.). Parce que les biologistes avaient eu un intérêt marqué pour les approches en sciences sociales et l'ethnologue avait bénéficié d'une expérience antérieure en recherches médicales sur la trypanosomiase américaine, l'exécution de ce glossaire garantissait à chacun l'emploi d'un vocabulaire commun auquel se référer en cas de doute.

L'objectif général de l'étude étant dirigé vers la recherche de facteurs de risque d'infestation des péridomiciles par les réduves, les chercheurs en sciences sociales se sont attachés à préciser les facteurs de risque issus de la structuration physique du péridomicile et des comportements humains tandis que les biologistes se sont centrés sur les facteurs de risque relevant de la biologie et du comportement des insectes (structure des populations, sources alimentaires, parasitisme, etc.). La comparaison et l'intégration des 
résultats issus de cette double approche ont permis in fine de mieux préciser la façon dont s'organise le péridomicile, vu comme un espace écologique particulier.

Il a fallu ensuite déterminer la taille de l'échantillon d'étude afin qu'il soit exploitable et répondre aux besoins propres de chacun. Les biologistes, pour leurs recherches, ont besoin d'un nombre suffisant d'insectes qu'ils récoltent donc en priorité là où ils pensent les trouver, en accord avec le véritable savoir-faire qu'ils ont développé au cours du temps. Les chercheurs en sciences sociales, adoptant dans ce cas particulier une approche épidémiologique, ont besoin d'un échantillonnage représentatif et suffisant de la population qu'ils souhaitent étudier. Le plan d'échantillonnage doit donc assurer aux biologistes une collecte optimale de matériel vivant (en fonction de la situation d'infestation locale) tout en restant un échantillon aléatoire autorisant un traitement statistique ultérieur. Dans l'exemple choisi, la recherche

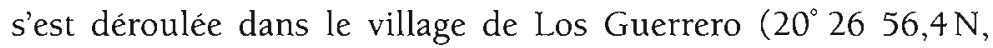
$103^{\circ} 5387,2 \mathrm{~W}$ ) qui comportait 348 unités d'habitation parmi lesquelles 100 , choisies aléatoirement, ont été visitées (BRENIÈRE et al. 2007; WALTER et al. 2007).

La recherche des facteurs de risque nécessite une bonne structuration des niveaux d'étude afin de repérer très exactement celui auquel chacun opère. Ainsi, dans ce cas particulier, nous avons procédé à une organisation des fiches d'enquête en quatre niveaux: la population d'insecte, l'habitat du vecteur représenté par une structure du péridomicile (c'est-à-dire des tas de brique, des piles de bois, des resserres et des poulaillers dans lesquels les triatomes s'installent), le péridomicile dans son ensemble et l'unité d'habitation (c'est-à-dire le péridomicile plus la maison). Chaque niveau a été étudié par concaténation des variables du niveau inférieur et par des variables qui lui sont propres. Au niveau le plus fin, l'étude des populations d'insectes définies par les individus collectés à un même endroit a précisé la distribution spatiale des insectes et les caractéristiques biogénétiques de chaque spécimen. Au niveau des éléments du péridomicile, la description du type de structure, des matériaux, de la position dans le péridomicile ainsi que l'étude de l'infestation par les triatomes ont permis d'étudier plus finement l'écologie péridomiciliaire de 
l'insecte. Au niveau du péridomicile, on a comptabilisé le type de structures et le nombre d'insectes qu'il renfermait mais on a aussi noté son aspect propre, s'il était fermé ou ouvert, s'il abritait des animaux ou des arbres, s'il était situé au centre du village ou en périphérie, cela afin de préciser les facteurs de risque à cette échelle. Au niveau de l'unité d'habitation, on a caractérisé les ménages d'un point de vue socioculturel et économique. Cette structuration rigoureuse des données collectées donne une certaine liberté à chaque discipline, libre de développer ultérieurement ses recherches au niveau qui est le plus pertinent pour elle. Mais elle permet aussi de relier des éléments biologiques (par exemple, la structuration des populations de vecteurs) à des éléments socioculturels (par exemple, les comportements de gestion du péridomicile par les ménages). Ce mode d'organisation, plus habituel aux chercheurs en sciences sociales qu'aux biologistes, impose à ces derniers des contraintes qui ne peuvent être acceptées sans une bonne vision de l'entreprise commune et des approches de chacun.

Le contenu des fiches d'enquête a été élaboré en commun de façon à répondre aussi bien aux questions d'ordre biologique (description de l'habitat des insectes, nombre d'insectes, etc.) que d'ordre socio-économique (présence d'animaux domestiques, superficie de la maison, nature des matériaux de construction, profil socio-économique du ménage, comportements). Au cours de ce processus, les biologistes ont apporté leur parfaite connaissance du terrain et de l'habitus des vecteurs, ce qui a permis d'adapter les questionnaires aux conditions locales et de les réduire aux questions essentielles. L'ethnologue, quant à lui, apporte un cadre théorique permettant de bien définir les espaces fonctionnels (zone d'activité agricole, zone résidentielle, zone de vie privée, péridomicile, etc.).

Si l'enquête de Los Guerrero a pu suggérer l'influence de certains comportements humains dans la pénétration des insectes au sein du péridomicile, elle ne traitait pas des facteurs de risque de diffusion des insectes de l'espace sauvage vers les espaces anthropisés. Ce thème est actuellement développé dans un autre programme qui fait également appel à une organisation de type interdisciplinaire dont quelques caractères sont abordés ci-dessous. 


\section{La mise en œuvre et la gestion des équipes}

Au cours de l'enquête de Los Guerrero, le travail de terrain a été exclusivement mené par les biologistes. L'année précédente, une enquête sérologique et une exploration cardiaque gratuites avaient été proposées à la population sous forme de volontariat. Cela a facilité l'étude postérieure des péridomiciles, car dès lors, les habitants ne percevaient pas le groupe comme des étrangers démarchant pour une cause commerciale ou autre. Toutefois, lors des premières visites le groupe, dont le nombre des intervenants a varié de quatre à sept et dont certains membres étaient déjà connus de la population, a été accompagné par une villageoise collaborant au centre de santé du village. Après avoir spécifié que le propos de notre visite était de trouver des triatomes à l'extérieur de la maison, les habitants étaient généralement très accueillants et tout à fait disposés à discuter avec la personne chargée de l'enquête familiale. Le reste du groupe se répartissait en trois activités: la production du plan à l'échelle de l'espace et le positionnement de chaque structure le composant, la caractérisation des différentes structures et des sites de recherche des insectes à l'aide de fiches standardisées et enfin la recherche proprement dite des insectes toujours effectuée par deux personnes (photo 3). Les membres du groupe évoluaient ensemble de maison en maison en prenant soin d'identifier correctement les flacons contenant les insectes en fonction de l'unité d'habitation, de la structure et du site de collecte. Tous les participants étaient conscients de l'ensemble des informations à collecter et s'aidaient mutuellement. Le nombre minimal d'intervenants a été de quatre personnes mais un nombre supérieur permet aussi de bavarder longuement et de répondre aux inquiétudes des habitants quant à la maladie de Chagas très mal connue chez les villageois.

D'autres enquêtes du même type, menées cette fois dans le Nord-Est brésilien, ont associé sur le terrain les chercheurs issus de différentes disciplines. Si chacun dispose bien de ses propres outils, complémentaires à ceux de ses collègues, et de la bonne volonté nécessaire à la mise en cuvre d'un travail interdisciplinaire, la rencontre des sciences biologiques et sociales sur un même échantillon de maisons doit néanmoins être minutieusement préparée. En effet, il n'est pas envisageable de passer à tour de rôle dans les foyers visités, 
Photo 3.

La recherche de triatomes dans un tas de briques.

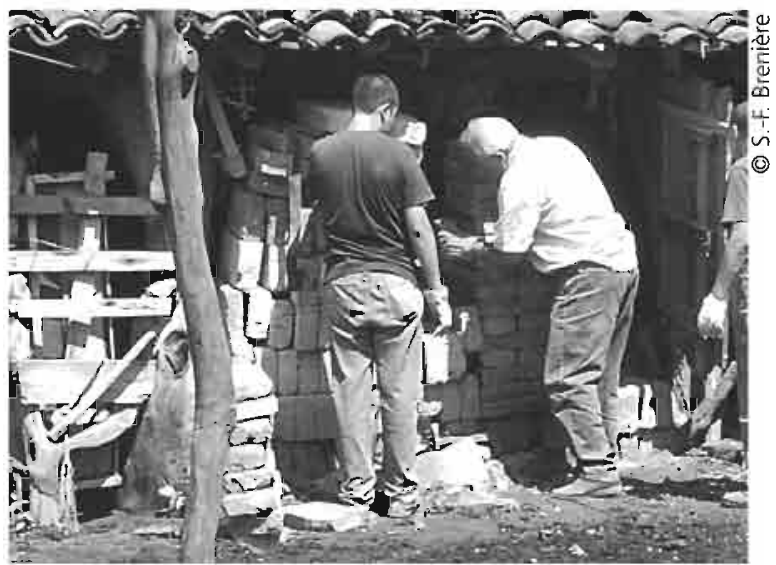

pour ne pas perturber de façon répétée l'organisation de vie des familles enquêtées. Les enquêtes entomologiques, c'est-à-dire la recherche et la collecte d'insectes dans les péridomiciles, et les enquêtes ethnologiques se sont donc déroulées conjointement. La présence des entomologistes (techniciens des services de lutte antivectorielle bien connus de la population) a d'ailleurs garanti une meilleure acceptation des ethnologues et de leurs entrevues. Il est également exclu de se présenter ensemble ou à tour de rôle, muni chacun de son questionnaire, face à une mère de famille quelque peu débordée. Ce point a été pris en compte dans la construction des questionnaires, non pas au niveau du contenu mais de la distribution des questions. La séparation, sur des feuilles différentes, des données d observation et des questions d'entretien garantit une séparation claire des tâches sur le terrain. L'entretien se déroule calmement un peu à l'écart, avec un seul interlocuteur, même si bien souvent d'autres membres de la famille y assistent (photo 4). Pendant ce temps le péridomicile est décrit par deux autres intervenants, l'un étant chargé d'élaborer un plan à l'échelle et l'autre de compléter les fiches descriptives du péridomicile.

Selon le caractère des populations, il est parfois difficile d'envahir à plus de quatre ou cinq l'espace privé des familles. Dans ce cas, il faut opter pour un nombre réduit d'enquêteurs au prix d'un allongement du temps de travail de chacun et de la durée totale 
Photo 4.

L'enquête familiale.

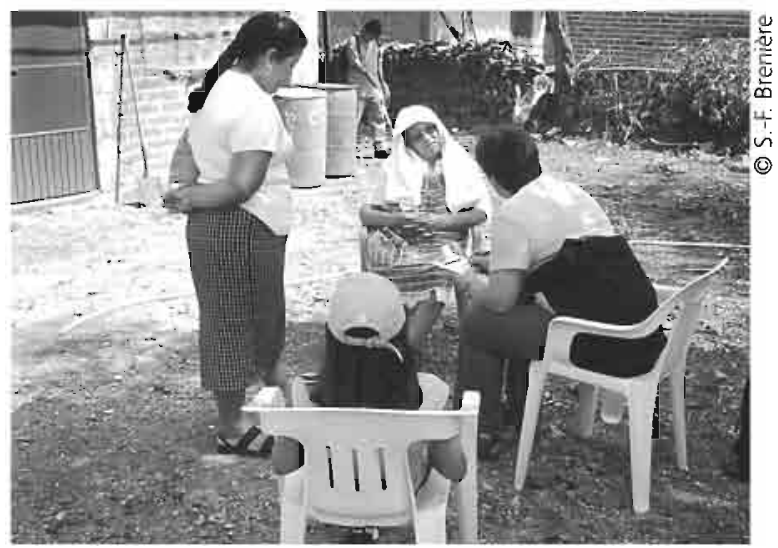

de l'enquête. À ce propos notons que la durée de présence d'un entomologiste dans un péridomicile dépend du nombre d'habitats potentiels pour les insectes et de la taille des colonies observées, tandis que celle des ethnologues est établie d'avance en fonction de l'importance de leur questionnaire ou de leur grille d'enquête. Les temps moyens d'enquête doivent donc être bien calculés et testés lors d'une pré-enquête pour programmer correctement la durée de l'intervention sur le terrain. Néanmoins, ce type de recherche engendre nécessairement un ralentissement du rythme habituel de chaque équipe ou, tout au moins, le sentiment que seul on irait plus vite. Il faut le savoir et préparer les enquêteurs à cette situation. L'expérience montre néanmoins que les habitants apprécient de discuter avec les visiteurs, aussi les enquêteurs doivent être agréables, bavards, pédagogues et doivent savoir, à tour de rôle, optimiser les temps d'attente pour établir ce contact amical avec les familles visitées. Tout cela peut paraître anodin et évident mais c'est souvent sur ce genre de détails que les programmes interdisciplinaires les mieux construits achoppent.

Si l étude des péridomiciles s'effectue conjointement, il n'en va pas de même lorsque les enquêtes se déroulent en dehors des unités d'habitation. Dans ce cas les sciences humaines ou des agronomes interviennent souvent les premiers, de façon autonome, afin de stratifier l'espace selon son usage en effectuant un relevé de parcelles à l'aide d'images satellites ou aériennes qui est ensuite validé sur le terrain. Les relevés argumentés des parcelles et les 
entretiens auprès des familles seront suivis de la collecte du matériel biologique qui se fait de façon raisonnée selon le plan d'occupation des sols précédemment établi. Il est évident que les temps de travaux doivent être là encore soigneusement évalués afin que les entomologistes disposent à temps des résultats des sciences humaines.

Nous avons présenté jusqu'à présent la façon dont sont menées les opérations communes entre les disciplines et nous remarquerons que l'approche reste biomédicale ou épidémiologique. C'est une phase préliminaire, descriptive, qui permet de mettre à jour des hypothèses et qui doit être suivie d'une phase plus analytique fondée sur des entretiens et des observations approfondies permettant de mettre à jour les représentations, les connaissances, les pratiques et les comportements face au vecteur et à la maladie. La méthode générale reste la méthode éco-épidémiologique sur laquelle nous nous sommes appuyés.

\section{L'interprétation des données}

Les données ayant été correctement saisies, en respectant la structuration des fiches d'enquêtes, le travail d'analyse a commencé. Il a été mené de façon conjointe, avec l'aide ultérieure d'une économiste pour les analyses multivariées, entrecoupé de nombreuses discussions interactives. Les données ont tout d'abord montré que la présence de tas de briques, de piles de bois ou d'une resserre augmentait le risque d'infestation des péridomiciles par Triatoma longipennis, l'espèce vectrice locale prédominante. Mais, après analyse des facteurs de risque aux différents niveaux d'appréhension, il est apparu que le risque prédominant ne résidait pas dans la simple présence de ces éléments, fréquents dans ce village, mais plutôt dans une organisation particulière du patio, caractérisée par la densité du bâti, l'encombrement de l'espace et la position centrale de l'unité d'habitation dans le village. Parallèlement, les biologistes ont montré que les repas de sang des insectes se prenaient majoritairement sur le rat, fréquent dans de nombreux péridomiciles, alors même que la présence d'animaux domestiques ne semblait pas corrélée à l'infestation par les réduves. D'autres facteurs suggéraient une participation 
des comportements humains, sans pouvoir être confirmée. Nous touchons là à une difficulté d'interprétation propre à ce genre d'enquête. La transformation des données qualitatives (comportements, pratiques, connaissances) en données quantitatives susceptibles d'être intégrées dans des SIG (système d'information géographique), des analyses statistiques ou des modèles mathématiques n'est pas chose aisée. Elle ne peut se faire qu'en plusieurs étapes et réclame un nombre suffisant de données à partir desquelles les variables sont déterminées. Dans le cas de Los Guerrero, nous avions par exemple remarqué que la présence d'un cheval favorisait l'infestation du péridomicile. Cet animal est utilisé dans la région pour se rendre dans les champs et surveiller les troupeaux. Est-il susceptible de rapporter des insectes dans la sellerie et de les introduire dans les péridomiciles? Ou bien signe-t-il l'appartenance de son propriétaire à un groupe particulier, socio-économique ou culturel, qui favoriserait par son comportement l'infestation du péridomicile? S'agit-il donc d'un facteur de risque, d'un indicateur de risque ou d'une simple association statistique non interprétable? C'est, entre autres, à partir de ce type d'association que les hypothèses de recherche sont posées puis développées ultérieurement, et souvent séparément, par chaque discipline.

Nous pouvons citer, comme autre exemple, une étude antérieure menée sur les facteurs de risque de ré-infestation des péridomiciles par Triatoma brasiliensis et Triatoma pseudomaculata, dans le nord-est du Brésil. Une première étude similaire dans sa conception à celle qui a été menée à Los Guerrero a montré que le mandacaru (Cereus jamacaru) était positivement associé à l'infestation des péridomiciles par T.brasiliensis. Il ne pouvait s'agir d'un facteur de risque direct puisque T. brasiliensis n'infeste pas le mandacaru. Dans un premier temps, il a simplement été conclu qu'il s'agissait d'un indicateur d'élevage intensif dans un milieu dégradé. En effet, cette cactée est cultivée par les éleveurs comme appoint alimentaire pour les troupeaux, en période de pénurie. Des études ultérieures menées par des biologistes ont alors montré que le prélèvement régulier des articles d'un mandacaru favorisait ses réitérations et lui donnait un port touffu dans lequel les oiseaux et de petits rongeurs pouvaient ensuite bâtir leur nid au sein duquel des populations de triatomes s'installaient. Mais elles appartenaient à des espèces différentes de T.brasiliensis, telle que Triatoma pseudomaculata 
(Emperaire et Romaña, 2006). Poursuivant leurs études les ethnologues ont ensuite montré que les éleveurs les plus jeunes et les plus actifs, ceux qui parcourent quotidiennement la caatinga à cheval pour rassembler et soigner leurs troupeaux sont ceux dont les péridomiciles sont les plus infestés par T.brasiliensis. L'hypothèse (qu'il faudrait confirmer par des recherches dirigées) est que ces éleveurs assurent indirectement un flux faible mais constant de vecteurs entre l'environnement et les unités d'habitation, flux en faveur du T.brasiliensis plus compétitif que T. pseudomaculata (POJO DE REGO et al., 2006). Se dessine ainsi un système d'interrelations homme/animal/vecteurs où chacun tient sa place, selon son habitus ou ses besoins, et qui n'aurait pas pu être mis à jour sans l'intervention de plusieurs disciplines. Dans ce système, la culture et l'exploitation du mandacaru est un facteur de risque d'infestation par T.pseudomaculata et un indicateur de risque d'infestation par T. brasiliensis. L'alternance des disciplines génère des questions, fournit des réponses et, en croisant les regards, révèle peu à peu les systèmes complexes des interrelations entre les différents composants biotiques et abiotiques des péridomiciles. Elle permet de cheminer du descriptif et du quantitatif, vers des analyses conceptuelles plus approfondies auxquelles participent pleinement les sciences biologiques et les sciences humaines. L'impératif, néanmoins, est de travailler sur des espaces communs, de s'astreindre à un dialogue permanent et d'accepter le partage des données. Parallèlement, les sciences humaines établissent le cadre général, social et culturel, dans lequel le cycle épidémiologique s'inscrit. Ces données et cette vision globale de la vie d'un village, d'un quartier ou d'un groupe social donnent bien souvent une foule d'informations utiles pour l'interprétation des données recueillies.

\section{La mise en forme et la publication des résultats}

Dans ce genre d'entreprise multidisciplinaire, l'organisation des groupes sur le terrain, le recueil de l'information et l'analyse des données se jouent un peu comme une enquête de détectives scientifiques. Au moment de la mise en forme des résultats en 
vue de leur publication, les règles du monde académique auquel nous appartenons reprennent pleinement leurs droits. Dans quelle revue publier? Quelle stratégie de publication adopter? La plupart des revues scientifiques sont encore, à l'heure actuelle, fortement mono-disciplinaires. Un article destiné à une revue d'obédience biologique n'acceptera pas les développements littéraires des sciences humaines et l'ethnologue se souvient de tout ce paragraphe décrivant le vaqueiro brésilien et relatant sa vie quotidienne, bien intéressant à ses yeux mais impropre à ce type de revue, qu'il a bien fallu supprimer de l'article en construction. D'un autre côté, une revue de sciences humaines accepte mal les démonstrations des biologistes lourdes de termes incompréhensibles pour ses lecteurs. La publication de ce type de travail initial s'appuie donc généralement sur des codes de langage biologique et c'est la stratégie que nous avons adoptée. En réalité, les nombreuses discussions que nous avons eues sur ce sujet nous ont amenées à un constat. La mise en œuvre d'une recherche multidisciplinaire et l'interprétation de ses résultats sont une entreprise de longue haleine qui se mène (et donc se publie) par étapes successives. Parlant de maladies parasitaires et fonctionnant dans un système de savoir médical de type occidental, il est légitime d'aborder le problème en suivant les codes de la pensée médicale occidentale. Les bases biologiques ainsi posées, les chercheurs en sciences humaines peuvent alors s'attacher à démêler la part des comportements humains et du système de représentations qui génèrent et modulent le système mis à jour en commun. Cette démarche aboutit à la confirmation ou la reformulation du système. C'est alors que les articles fondés sur des codes de langages plus littéraires peuvent être produits, en collaboration avec des biologistes.

Dans une première étape les sciences humaines apportent donc une base structurante sur laquelle s'inscrivent les recherches biologiques et une vision d'ensemble du contexte social et culturel. À ce stade, la publication des résultats tend à révéler la nature et le fonctionnement du système vectoriel et suit les codes de présentation et de langage du savoir biologique. Dans une autre étape, les sciences humaines intègrent les arcanes des modes de pensée et des comportements de l'acteur humain afin de mettre à jour la façon dont s'est construit le système vectoriel et la façon dont il se module. Les codes de présentation et de langage sont ceux du 
savoir anthropologique. Ils apportent une ouverture supplémentaire sur les interrelations entre les éléments du monde vivant et pose des questions nouvelles aux biologistes et aux anthropologues.

\section{Remerciements}

Le travail mené à Los Guerrero est porté par une collaboration interactive entre les chercheurs de l'IRD de plusieurs disciplines et les chercheurs de l'université de Guadalajara dont nous tenons tout particulièrement à remercier le Dr Felipe Lozano Kasten, médecin en santé publique, Ezequiel Magallón Gastélum, chercheur entomologiste et Margarita Soto, biologiste.

\section{Références bibliographiques}

Bosseno M.-F., Garcia L.-S., Baunaure F., Gastelum E.-M., Gutierrez M.-S., Kasten F.-L., Dumonteil. E., Breniěre S.-F, 2006 Identification in triatomine vectors of feeding sources and Trypanosoma cruzi variants by heteroduplex assay and a multiplex miniexon polymerase chain reaction. Am. J. Trop. Med. Hyg., 74 (2) : 303-305.

Brenière S.-F, Bosseno M.-F, Magallon-Gastelum E., Castillo Ruvalcaba E.-G., Gutierrez M.-S., Montaño luna E.-C., Basulto J.-T., Mathieu-Daude F, Walter A., Lozano-Kasten F, 2007 - Peridomestic colonization of Triatoma longipennis (Hemiptera, Reduviidae) and Triatoma barberi (Hemiptera, Reduviidae) in a rural community with active transmission of Trypanosoma cruzi in Jalisco state, Mexico. Acta tropica, 101 (3): 249-57.

EMPeraire L., Romaña C., 2006 - Triatominae and Cactaceae : a risk for the transmission of the American trypanosomiasis in the peridomicilary space (Northeast Brazil). Parasite, 13 (2): 171-178.

Ferreira L.-F., Britto C., Cardoso M.-A., Fernandes O., Reinhard K., ARAujo A., 2000 - Paleoparasitology of Chagas disease revealed by infected tissues from Chilean mummies. Acta tropica, 75: 79-84.

Guhl F., Jaramillo C., Yockteng R., Vallejo G-A., CArdenas-Arroyo F, 1997 - Trypanosoma cruzi DNA in human mummies. Lancet, 349 (9062): 1370.

Magallón-Gastélum E., Lozano-Kasten F., Gutierrez M.-S., Flores-Perez A., SANCHEZ B., EsPinoza B., Bosseno M.-E., BreniÈre S.-F, 2006 - Epidemiological risk for Trypanosoma cruzi transmission by species of Phyllosoma complex in the occidental part of Mexico. Acta Tropica, 97 (3) : 331-338. 
Pojo de Rego I., Walter A., Ferreira A-J. Rangel M., GirardFerreira E., Noireau F, 2006 - Peridomestic structures and farming activity on triatomine infestation, Parasite, 13 (3): 237-243.

Walter a., lozano-Kasten F., Bosseno M.-F, Ruvalcaba E.-G., Gutierrez M.-S., Luna C-E., Baunaure F, Phélinas P., MagallónGASTÉlum E., BREnIĖRe S.-F., 2007 - Peridomicilary habitat and risk factors for Triatoma infestation in a rural community of the Mexican occident. Am. J. Trop. Med. Hyg., 76 (3): 508-15.

Yeo M., Acosta N., Llewellyn M., Sanchez H., Adamson S., Miles G.-A., Lopez E., Gonzalez N., Patterson J.-S., Gaunt M.-W., De Arias A.-R., MILES M.-A., 2005 - Origins of Chagas disease: Didelphis species are natural hosts of Trypanosoma cruzi I and armadillos hosts of Trypanosoma cruzi II, including hybrids. Int. J. Parasitol., 35: 225-233. 


\section{La nécessité du recours à l'interdisciplinarité pour étudier le risque de ré-émergence du paludisme en Camargue}

\section{Nicolas PONÇON, Céline TOTY \\ Katrin LANGEWIESCHE, Alain DERVIEUX \\ Didier FONTENILLE}

Notre planète subit depuis ces quatre dernières décennies des évolutions climatiques, écologiques, démographiques et économiques qui peuvent favoriser la résurgence des principales maladies à transmission vectorielle dont l'impact sanitaire, socio-économique et politique est important. Dans ce cadre, le paludisme, endémique autrefois en Europe et éradiqué au milieu du $\mathrm{XX}^{\mathrm{e}}$ siècle, réapparaît sous les feux de l'actualité scientifique européenne. Des foyers ont été répertoriés ces dernières années aux frontières de l'Europe en Azerbaidjan, Turquie et Géorgie où cette maladie avait disparu après la Seconde Guerre mondiale (WHO, 2007). Quelques cas isolés de transmission autochtone ont été reportés ces dernières années en Europe, poussant les scientifiques à s'intéresser à nouveau à cette maladie et à s'interroger sur le risque de ré-émergence du paludisme en Europe et notamment en France (WHO, 2007 ; BaLdARI et al., 1998 ; KRUGER et al., 2001 ; CUADROS et al., 2002 ; KAMPEN et al., 2003 ; ARMENGAUD et al., 2006 ; DOUDIER et al., 2007). 


\section{Le contexte}

Le paludisme est une parasitose due à un hématozoaire du genre Plasmodium. Quatre espèces peuvent toucher les humains: $P$. falciparum, $P$. vivax, $P$. malariae et $P$. ovale, causant annuellement 300 millions d'accès et plus de 1 million de décès.

Le paludisme sévissait en France métropolitaine dans de nombreuses régions marécageuses comme les Landes, la Dombes, la Bretagne, l'Alsace, la Camargue, la Sologne, le Roussillon et dans certaines zones de Corse. Cette maladie y était endémique jusqu'au début du XX $X^{e}$ siècle et la transmission de l'hématozoaire du paludisme était assurée par des espèces locales de moustique, du genre Anopheles. Plusieurs facteurs entraînèrent la régression puis la disparition définitive de cette maladie. L'amélioration des conditions de vie et le recours systématique au traitement par la quinine eurent un impact majeur. La lutte antivectorielle et l'assèchement des marais par la mise en valeur des terres contribuèrent également à cette régression. La Camargue fut le dernier foyer actif en France continentale et il s'éteignit à la fin de la Seconde Guerre mondiale (SAUTET, 1944). De nos jours, les seuls cas répertoriés de paludisme en France sont des cas de paludisme importé, c'est-à-dire des personnes contaminées au cours d'un voyage en zone endémique (majoritairement en Afrique de l'Ouest) et qui expriment des symptômes cliniques de retour en France. Ainsi, 3442 cas de paludisme importé ont été notifiés en 2004, correspondant à 6109 cas estimés. Quelques rares cas accidentels (greffe, transfusion...) ont été reportés également (LEGROS et al., 2006).

Malgré la diminution des populations de moustiques suite à l'assèchement de certaines zones marécageuses au cours du siècle dernier et aux opérations de démoustication actuelles, les Anopheles sont toujours présents en France et en populations importantes à certains endroits, notamment en Camargue. Cette situation est caractérisée par l'expression "anophélisme sans paludisme". Dans ce cadre, l'hypothèse de la reprise de cycles vectoriels autochtones impliquant les espèces Anopheles locales en Camargue suite à l'introduction régulière de Plasmodium par les différents flux humains (touristes, travailleurs saisonniers...) mérite d'être considérée. L'évaluation de ce risque implique l'étude du système vectoriel potentiel, dont la compréhension nécessite une approche globale s'intéressant à 
l'homme, hôte unique et réservoir de Plasmodium, aux espèces d'Anopheles, moustiques vecteurs potentiels, et à l'environnement dans lequel humains et Anopheles évoluent. Létude du cycle vectoriel potentiel du paludisme en Camargue se pose de la manière suivante:

- Existe-t-il des moustiques capables de transmettre les Plasmodium en Camargue?

- Ces moustiques piquent-ils les humains?

- Ces moustiques rencontrent-ils des porteurs de Plasmodium en Camargue?

Afin de trouver des réponses à ces questions et d'appréhender le risque de ré-émergence du paludisme en Camargue, l'interdisciplinarité est indispensable. Différentes disciplines des sciences sociales et des sciences biologiques sont mobilisées.

Dans l'exemple présenté dans ce texte, les données entomologiques, du milieu et celles issues des sciences sociales, actuelles et historiques, ont été obtenues par différentes équipes de l'Institut de recherche pour le développement (IRD), du Centre national de la recherche scientifique (CNRS), du Centre de coopération internationale en recherche agronomique pour le développement (Cirad), de l'Entente interdépartementale pour la démoustication (EID-Méditerranée), de l'Institut universitaire professionnel, Environnement technologies et société de l'université de Provence (IUP-ENTES), du Centre national de référence de l'épidémiologie du paludisme d'importation et autochtone (CNREPIA) et des hôpitaux régionaux.

\section{Quelques éléments sur le cycle du Plasmodium et ses vecteurs}

Pour assurer sa descendance, la femelle moustique doit effectuer des repas de sang. Le choix de l'hôte est conditionné par la biologie de l'espèce: anthropophile strict, mammophile, ornithophile ou opportuniste. À la suite de la piqûre, la femelle entre en phase de repos pendant laquelle la digestion du sang assure le développement des œufs. Arrivés à maturité, les œufs sont pondus sur un plan 
d'eau, le choix de celui-ci dépendant de l'espèce (préférence stricte pour certains milieux, ubiquité...). Après la ponte, la femelle recherche à nouveau un hôte, réalise un repas de sang, effectue un nouveau cycle et ainsi de suite jusqu'à sa mort (la durée de vie des moustiques dépendant des facteurs climatiques et de l'accès à la nourriture). La période séparant deux piqûres définit le cycle trophogonique. Les œufs éclosent et libèrent des larves aquatiques qui se développent et évoluent en nymphes qui se métamorphosent en adultes ailés mâles ou femelles.

La femelle peut s'infecter en réalisant un repas de sang sur un homme porteur du stade gamétocyte des Plasmodium. Les parasites réalisent alors un cycle, appelé cycle sporogonique ou extrinsèque, au cours duquel ils migrent de l'intestin aux glandes salivaires du moustique. Le stade sporozoite des Plasmodium sera injecté à un humain lors d'une piqûre ultérieure. La durée de ce cycle varie d'une dizaine à une trentaine de jours en fonction de l'espèce plasmodiale et de la température (au-delà d'une température seuil au-dessous de laquelle le cycle est bloqué: $18^{\circ} \mathrm{C}$ pour $P$. falciparum et $15-16^{\circ} \mathrm{C}$ pour $P$. vivax (MACDONALD, 1957). De plus, des facteurs génétiques de la femelle Anopheles déterminent la possibilité pour le Plasmodium de réaliser complètement le cycle extrinsèque: cette compatibilité entre le parasite et le vecteur est appelée compétence vectorielle.

La sommation de ces différents facteurs (préférences trophiques, durée du cycle trophogonique, longévité des femelles, durée du cycle extrinsèque) combinés à l'abondance des femelles détermine la capacité vectorielle qui renvoie à l'efficacité du vecteur dans un environnement donné.

\section{Le risque de ré-émergence du paludisme en Camargue: une approche interdisciplinaire}

\section{Existe-t-il des moustiques capables de transmettre des Plasmodium en Camargue?}

Un moustique sera un vecteur potentiel s'il est présent, voire abondant dans un milieu donné, s'il pique les humains, si sa 
durée de vie dépasse celle du cycle sporogonique et s'il est compétent vis-à-vis de Plasmodium. L'étude de ces critères nécessite la mobilisation de méthodologies et compétences variées et complémentaires.

\section{Étude du milieu}

La Camargue est une zone humide qui couvre le delta du Rhône qui s'étend du Grand-Plan-du-Bourg à l'est au canal de Pécais à l'ouest. Marais, étangs et rizières en constituent une part importante. L'ensemble de cette région est alimenté en eau douce depuis le Rhône par un réseau dense de canaux d'irrigation et de drainage qui sert essentiellement à l'alimentation en eau des parcelles rizicoles. La disponibilité de la ressource a entraîné son utilisation pour l'alimentation des milieux «naturels» (marais, étangs) à des fins cynégétiques, d'élevage, de conservation de la nature, ou de récolte du roseau. Le réseau de canaux de drainage permet de gérer selon les besoins l'ensemble de ces milieux humides et contribue à l'artificialisation du fonctionnement hydrologique du delta et, in fine, à une tendance à la permanence de l'eau tout au long de l'année dans les milieux naturels. Cette région est également caractérisée par une nappe phréatique salée, peu profonde et dont les effets influencent la salinité des sols. Celle-ci dépend de la distance par rapport à la côte et de la fréquence de submersion du sol en eau douce. La flore des marais dépend de la salinité et de la xérophilie. Ces deux facteurs conjoints, combinés à la pression de pâturage, déterminent différentes associations végétales et donc différents types de marais. La Camargue est ainsi couverte d'une juxtaposition de milieux différents (DERvieuX et al., 2002 ; AZNAR et al., 2003).

Dans ce contexte fortement anthropisé, la compréhension du milieu passe nécessairement par la compréhension des interactions homme/milieu. De plus, la caractérisation et la cartographie de la Camargue pour cette étude ont nécessité d'inclure la composante vecteur dans la réflexion pour identifier les déterminants pertinents. Finalement, botanique, sciences sociales et biologie ont interagi pour produire une cartographie mobilisant relevés de terrain, images satellites et systèmes d'informations géographiques. 


\section{Inventaire et dynamique des populations d'Anopheles en fonction du climat et du milieu}

Des prospections entomologiques poussées ont été menées régulièrement dans deux zones représentatives de Camargue, dans le secteur de la Tour-Carbonnière à l'ouest, celui des marais du Vigueirat à l'est (fig. 1). Différentes méthodes de captures d'adultes

Figure 1.

Zones de prospection des Anopheles de Carmargue.

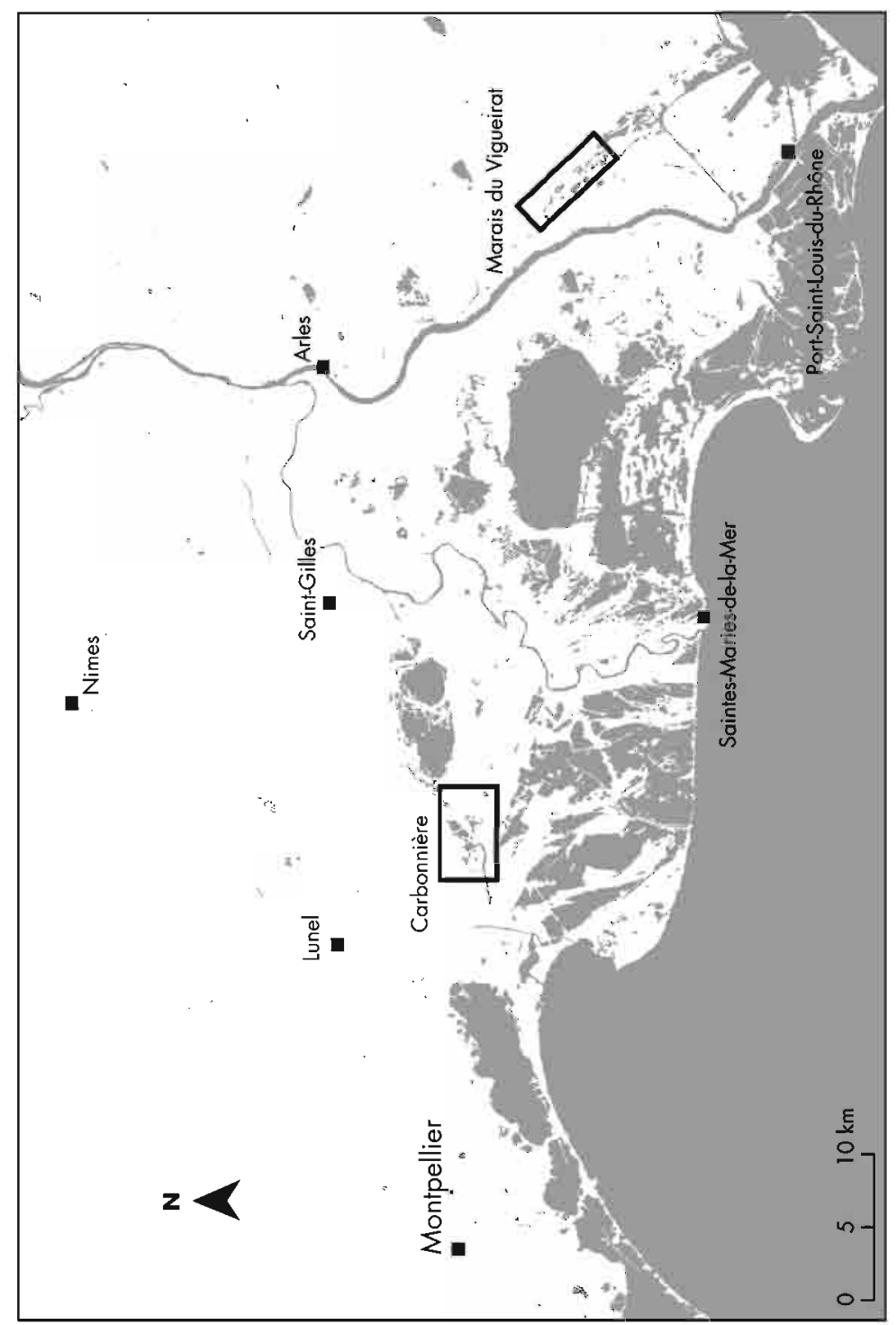


et de larves ont été utilisées en différents points de mars à décembre 2005, en lien avec différents milieux. La détermination des différentes espèces a nécessité dans certains cas, le recours aux outils de biologie moléculaire (PROFT et al., 1999). Plus de 680000 moustiques ont été récoltés dont 131050 Anopheles. Quatre espèces d'Anopheles ayant des variations spatiales d'abondance ont été répertoriées: An. hyrcanus, An. melanoon,

Figure 2a.

Dynamique saisonnière d'An. melanoon et An. hyrcanus à la Carbonnière en 2005.

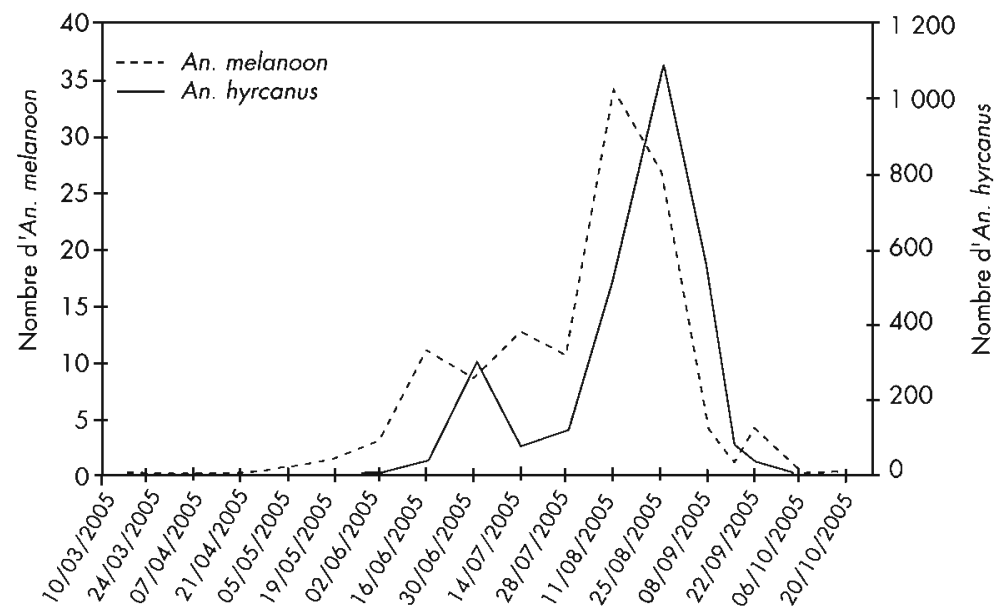

Figure $2 b$.

Dynamique saisonnière d'An. melanoon, An. algeriensis et An. hyrcanus au marais du Vigueirat en 2005.

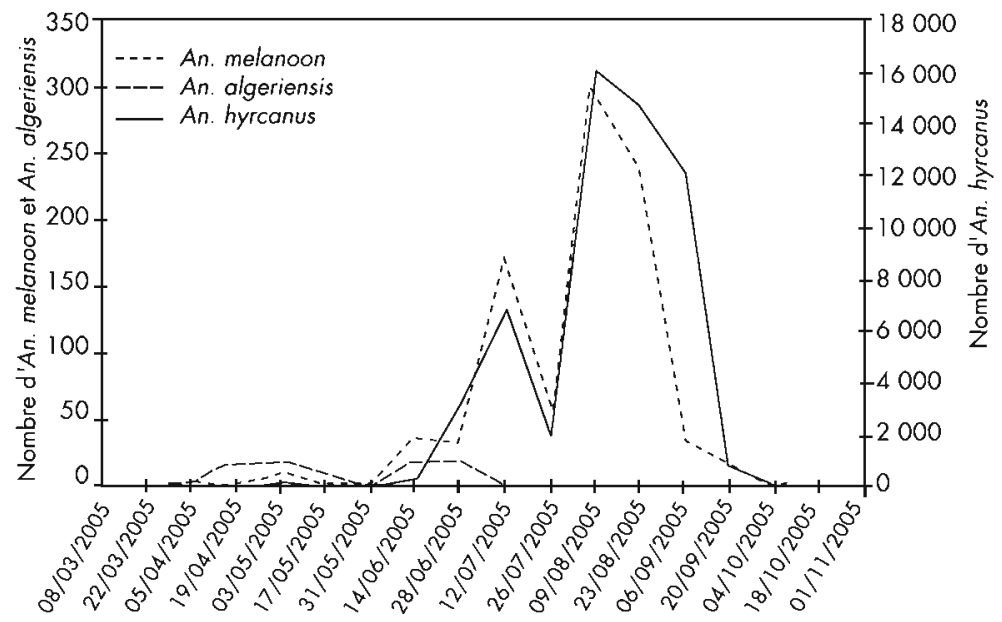


Figure 3.

Abondance d'An. melanoon et An. hyrcanus

en fonction des points et milieux de capture.

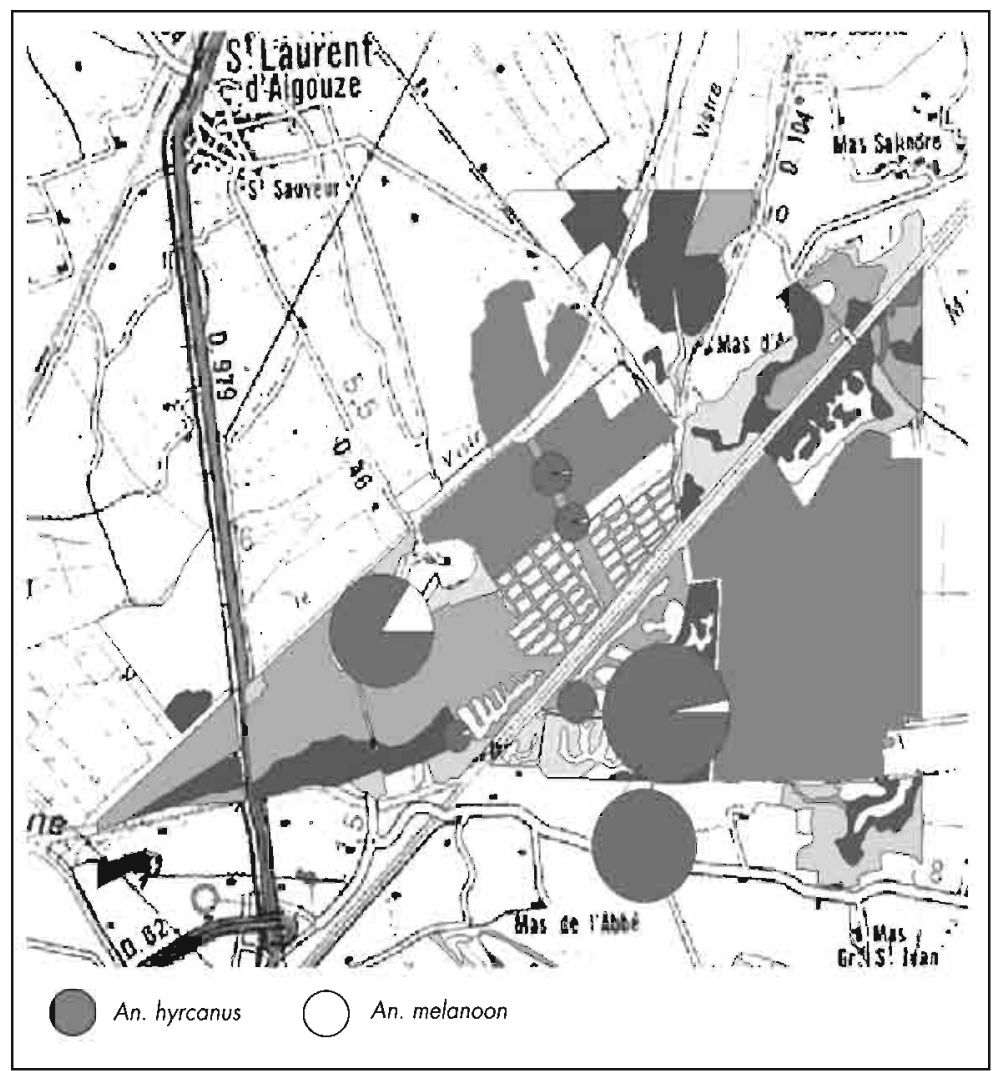

An. algeriensis, An. atroparvus. Les dynamiques de populations sont différentes: An. hyrcanus et An. melanoon sont présents en été, tandis que An. algeriensis et An. atroparvus, peu abondants, sont présents respectivement au printemps et en hiver (PONÇON et al., 2007).

\section{Longévité des anophèles}

À l'échelle d'une population, la durée de vie des femelles Anopheles est estimée à partir du taux de parturité de la population (proportion de femelles ayant pondu au moins une fois), qui a été déterminé après dissection des ovaires de femelles capturées (Detinova, 1962). 


\section{Compétence vectorielle}

Des expériences de transmissions expérimentales ont été menées en laboratoires sécurisés. Des populations locales d'Anopheles ont été gorgées sur des membranes artificielles leur permettant d'ingérer du sang infecté. Le suivi des infections a permis d'étudier la compétence des espèces Anopheles françaises vis-à-vis des souches de $P$. falciparum tropicaux. Ce travail a démontré que, si la compétence des Anopheles de Camargue vis-à-vis des $P$. falciparum tropicaux est faible, elle n'est pas pour autant nulle.

Les résultats concernant la présence des vecteurs potentiels, leur longévité et leur compétence vectorielle, combinés à la cartographie déterminent la répartition spatio-temporelle des espèces anophèles susceptibles de transmettre des Plasmodium. Le milieu et les facteurs climatiques permettent de détecter les zones entomologiquement à risque à l'intérieur desquelles le contact entre ces Anopheles et les humains doit être étudié.

\section{Ces moustiques piquent-ils les humains?}

L'un des éléments clés du cycle de transmission du paludisme réside dans le contact entre l'homme et les Anopheles - contact indispensable pour que le moustique s'infecte et pour assurer la transmission. Deux composantes interactives déterminent ce contact: le comportement trophique des Anopheles (c'est-à-dire l'hôte sur lequel et la période pendant laquelle seront pris préférentiellement les repas de sang, ainsi que leur fréquence) et la présence ainsi que les comportements des humains.

Le comportement trophique est déterminé par trois facteurs: la préférence d'hôte, la durée du cycle trophogonique et le rythme journalier d'activité (heures préférentielles des repas de sang: crépuscule, nuit...). L'efficacité d'un vecteur dépend du choix de son hôte (un bon vecteur de paludisme doit piquer l'homme) et de la fréquence des piqûres (plus le cycle trophogonique est court, plus le vecteur piquera fréquemment). Le rythme journalier d'activité du moustique influe sur les possibilités de contacts avec l'hôte. 
De plus, quand bien même un moustique serait disposé à piquer un homme, il est nécessaire qu'un humain soit disponible pour ce repas de sang, c'est-à-dire qu'il soit présent dans l'environnement du moustique et qu'il ne se protège pas suffisamment contre les piqûres.

Afin de trouver une réponse à la question «ces moustiques piquent-ils l'homme?", une étude des contacts homme/vecteurs en fonction du comportement trophique des Anopheles, de la distribution spatio-temporelle et du comportement des humains s'imposait.

\section{Étude du comportement trophique des anophèles}

L'étude du choix de l'hôte a été menée par des piégeages sur différents hôtes (homme et cheval) et par l'analyse des repas de sang des femelles Anopheles capturées gorgées [cette analyse fait appel à une technique de type ELISA (BEIER et al., 1988)]. Des différences nettes ont été observées dans le comportement des espèces, An. hyrcanus étant très anthropophile tandis que An. melanoon, piquant rarement l'homme, est très zoophile.

Tableau I.

Captures sur appât cheval et humain

au marais du Vigueirat en 2005

(données compilées de 3 nuits de capture

réalisées chacune sur 1 cheval et 3 humains)

\begin{tabular}{|lcc|}
\hline & $\begin{array}{c}\text { Appât } \\
\text { cheval }\end{array}$ & $\begin{array}{c}\text { Appât } \\
\text { humain }\end{array}$ \\
\hline An. melanoon & 1411 & 10 \\
\hline An. hyrcanus & 856 & 7062 \\
\hline
\end{tabular}

Les captures d'adultes ont révélé une activité forte des femelles An. hyrcanus en début de nuit tandis que An. melanoon semble plus actif en seconde partie de nuit.

La durée du cycle trophogonique est en général de 4-5 jours en France. 
Tableau 2.

Analyse des repas de sang des Anopheles femelles capturées gorgées dans des sites refuges des moustiques

(les repas sur homme ont été recherchés au même titre que les autres hôtes: aucun Anopheles n'a présenté un repas de sang pris sur humain)

\begin{tabular}{|c|c|c|c|c|c|c|c|c|}
\hline \multirow[t]{2}{*}{ Espèce } & \multirow[t]{2}{*}{ Zone d'étude } & \multirow{2}{*}{$\begin{array}{l}\text { Nombre } \\
\text { de moustiques }\end{array}$} & \multicolumn{6}{|c|}{ Origine du repas de sang } \\
\hline & & & Cheval & Bovin & Sanglier & Mixte & Chien & Autres hôtes \\
\hline \multirow[t]{2}{*}{ An. melanoon } & Carbonnière & 142 & 95 & 0 & 0 & 2 & 0 & 4 \\
\hline & Marais du Vigueirat & 84 & 67 & 8 & 8 & 0 & 1 & 7 \\
\hline \multirow[t]{2}{*}{ An. atroparvus } & Carbonnière & 12 & 10 & 0 & 0 & 0 & 0 & 0 \\
\hline & Marais du Vigueirat & 3 & 3 & 0 & 0 & 0 & 0 & 0 \\
\hline \multirow[t]{2}{*}{ An. maculipennis complex } & Carbonnière & 11 & 9 & 0 & 0 & 0 & 0 & 0 \\
\hline & Marais du Vigueirat & 6 & 2 & 2 & 2 & 0 & 0 & 0 \\
\hline \multirow[t]{2}{*}{ An. hyrcanus } & Carbonnière & 1 & 1 & 0 & 0 & 0 & 0 & 0 \\
\hline & Marais du Vigueirat & 8 & 7 & 1 & 1 & 0 & 0 & 0 \\
\hline
\end{tabular}




\section{Étude de la distribution spatio-temporelle des hommes}

La répartition spatio-temporelle des humains a été répertoriée par des enquêtes socio-anthropologiques, en caractérisant la population et ses activités en lien avec le milieu sur une des deux zones de capture de moustiques. Ce travail a été réalisé à l'échelle annuelle et journalière - le choix des critères caractérisant la population étant lié à la composante vecteur (Langewiesche, Rapport «Eden-volet sciences sociales", 2005). Cette étude reporte des variations temporelles importantes dues aux activités touristiques dans cette zone. On peut compter 340 personnes par jour en hiver et autour de 2000 en été. La distribution spatiale des humains varie également dans l'année et dans la journée en fonction de leurs activités (agricoles ou touristiques). Cette étude permet d'identifier des groupes (riziculteurs, viticulteurs, coupeurs de roseaux, éleveurs et chasseurs) exposés aux Anopheles, sur la

Figure 4.

Répartition spatiale des activités agricoles et touristiques dans la zone d'étude de la Carbonnière en 2005.

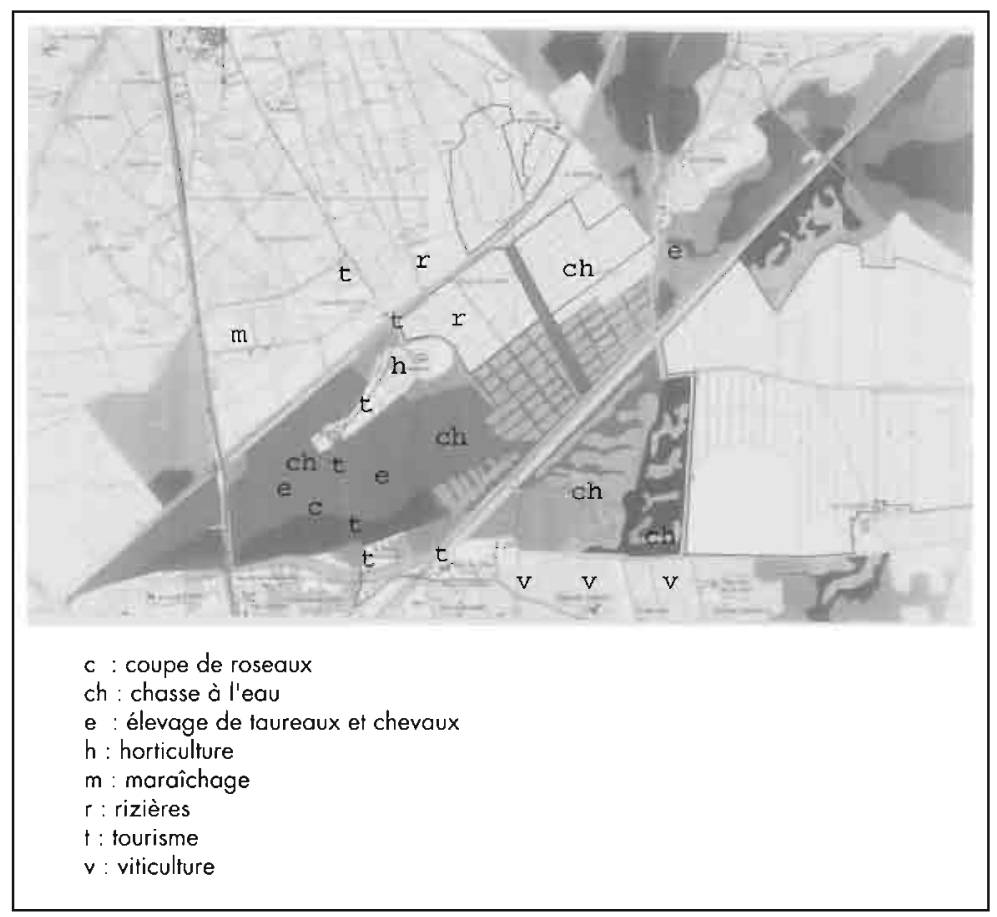


Tableau 3.

Estimation du nombre de personnes présentes dans la zone d'étude de la Carbonnière par jour pour les différents mois de l'année 2005

\begin{tabular}{|c|c|c|c|c|c|c|c|c|c|c|c|c|}
\hline Mois & 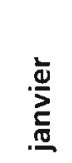 & 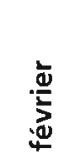 & $\stackrel{n}{\frac{n}{\pi}}$ & $\sum_{\bar{\pi}}^{\bar{\Sigma}}$ & $\stackrel{\bar{\pi}}{\Xi}$ & $\stackrel{\underline{s}}{\underline{2}}$ & 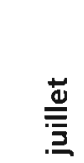 & 芯 & 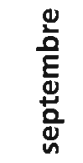 & 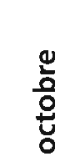 & 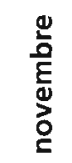 & $\begin{array}{l}\frac{0}{0} \\
\frac{0}{E} \\
\frac{d}{0}\end{array}$ \\
\hline $\begin{array}{l}\text { Habitants } \\
\text { permanents }\end{array}$ & 200 & 200 & 200 & 200 & 200 & 200 & 200 & 200 & 200 & 200 & 200 & 200 \\
\hline \multicolumn{13}{|l|}{ Travailleurs réguliers } \\
\hline $\begin{array}{l}\text { horticulture } \\
\text { viticulture }\end{array}$ & $\begin{array}{r}10 \\
8\end{array}$ & $\begin{array}{r}10 \\
8\end{array}$ & $\begin{array}{r}10 \\
8\end{array}$ & $\begin{array}{r}10 \\
8\end{array}$ & $\begin{array}{r}10 \\
8\end{array}$ & $\begin{array}{r}10 \\
8\end{array}$ & $\begin{array}{r}10 \\
8\end{array}$ & $\begin{array}{r}10 \\
8\end{array}$ & $\begin{array}{r}10 \\
8\end{array}$ & $\begin{array}{r}10 \\
8\end{array}$ & $\begin{array}{r}10 \\
8\end{array}$ & $\begin{array}{r}10 \\
8\end{array}$ \\
\hline maraîchage, Copam & 5 & 5 & 5 & 5 & 5 & 5 & 5 & 5 & 5 & 5 & 5 & 5 \\
\hline maraîchage, Soproma & 80 & 80 & 80 & 80 & 80 & 80 & 80 & 80 & 80 & 80 & 80 & 80 \\
\hline $\begin{array}{l}\text { riziculture } \\
\text { Travailleurs réguliers }\end{array}$ & 2 & 2 & 2 & 2 & 2 & 2 & 2 & 2 & 2 & 2 & 2 & 2 \\
\hline Total & 105 & 105 & 105 & 105 & 105 & 105 & 105 & 105 & 105 & 105 & 105 & 105 \\
\hline \multicolumn{13}{|l|}{ Suivi scientifique } \\
\hline personnel EID & 3 & 3 & 3 & 3 & 3 & 3 & 3 & 3 & 3 & 3 & 3 & 3 \\
\hline $\begin{array}{l}\text { personnel Scamandre } \\
\text { Suivi scientifique }\end{array}$ & 5 & 5 & 5 & 5 & 5 & 5 & 5 & 5 & 5 & 5 & 5 & 5 \\
\hline Total & 8 & 8 & 8 & 8 & 8 & 8 & 8 & 8 & 8 & 8 & 8 & 8 \\
\hline \multicolumn{13}{|c|}{ Travailleurs saisonniers } \\
\hline viticulture & 0 & 0 & 0 & 0 & 0 & 0 & 0 & 20 & 20 & 20 & 5 & 5 \\
\hline élevage & 0 & 0 & 0 & 6 & 6 & 6 & 6 & 6 & 6 & 0 & 0 & 0 \\
\hline coupe du roseau & 1 & 1 & 1 & 0 & 0 & 0 & 10 & 10 & 0 & 0 & 0 & 3 \\
\hline maraîchage, Copam & 10 & 10 & 20 & 20 & 60 & 60 & 40 & 40 & 10 & 10 & 10 & 10 \\
\hline $\begin{array}{l}\text { maraîchage, Soproma } \\
\text { Travailleurs saisonniers }\end{array}$ & 0 & 0 & 0 & 40 & 40 & 40 & 0 & 0 & 0 & 0 & 0 & 0 \\
\hline Total & 11 & 11 & 21 & 66 & 106 & 106 & 56 & 76 & 36 & 30 & 15 & 18 \\
\hline \multicolumn{13}{|l|}{ Activités touristiques } \\
\hline visite de rizière & 0 & 0 & 0 & 0 & 4 & 1 & 17 & 17 & 17 & 0 & 0 & 0 \\
\hline visite en $4 \times 4$ & 0 & 0 & 0 & 0 & 5 & 5 & 20 & 20 & 0 & 0 & 0 & \\
\hline visite guidée & 0 & 0 & 1 & 1 & 1 & 1 & 2 & 2 & 1 & 1 & 0 & 0 \\
\hline visite en péniche & 0 & 0 & 0 & 0 & 200 & 600 & 800 & 800 & 800 & 200 & 0 & 0 \\
\hline camping & 0 & 0 & 0 & 100 & 100 & 100 & 600 & 600 & 100 & 0 & 0 & 0 \\
\hline $\begin{array}{l}\text { hôtels/gîtes } \\
\text { Activités touristiques }\end{array}$ & 0 & 0 & 0 & 50 & 50 & 300 & 300 & 300 & 300 & 0 & 0 & 0 \\
\hline Total & 0 & 0 & 1 & 151 & 360 & 1007 & 1739 & 1739 & 1218 & 201 & 0 & 0 \\
\hline $\begin{array}{l}\text { Activités de loisirs } \\
\text { chasse/pêche }\end{array}$ & 13 & 13 & 0 & 0 & 0 & 0 & 0 & 13 & 13 & 13 & 13 & 13 \\
\hline Total & 337 & 337 & 335 & 530 & 779 & 1426 & 2108 & 2141 & 1580 & 557 & 341 & 344 \\
\hline
\end{tabular}


base de leurs activités dans des milieux favorables aux Anopheles, ou proches de ceux-ci, et à des horaires correspondant à l'activité des vecteurs potentiels. De plus, les touristes, en particulier les étrangers, sont également très exposés aux piqûres de moustiques, moins par leurs pratiques que par leurs comportements peu adaptés à la nuisance.

\section{Étude des comportements humains}

Les premiers résultats mettent clairement en évidence des différences d'exposition des différents groupes humains face aux piqûres des moustiques. Les résidents se protègent mieux des moustiques que ne le font les touristes et visiteurs peu familiarisés à cette nuisance.

Les comportements spécifiques de chaque groupe d'humains combinés aux variations spatio-temporelles de leur présence permettent d'établir une carte des humains «disponibles» pour les repas de sang des Anopheles, carte qui varie au cours de la journée et de l'année.

Le croisement des données concernant les moustiques et les humains permet ainsi de cartographier des zones de contacts entre humains et vecteurs en Camargue, définissant ainsi des zones de transmission potentielle. À l'intérieur de ces zones, la rencontre d'un Anopheles capable de transmettre les Plasmodium avec un homme porteur de parasites pourra initier un cycle de transmission. Il est donc nécessaire de déterminer la distribution spatio-temporelle des humains porteurs de Plasmodium en Camargue.

\section{Ces moustiques rencontrent-ils des Plasmodium en Camargue ?}

La transmission autochtone du paludisme ayant cessé en Camargue depuis le milieu du XX $\mathrm{XX}^{\mathrm{e}}$ siècle, les seules sources actuelles de parasites sont représentées par les cas de paludisme importé ou les porteurs chroniques de parasites (après une première contamination par les Plasmodium, on observe un portage de parasites dans l'organisme qui varie de quelques mois à plusieurs années en fonction de l'espèce plasmodiale impliquée et d'une 
éventuelle immunité). Ces sources de parasites dépendent exclusivement des mouvements de populations pour lesquels les déterminants sociaux et économiques sont importants.

\section{Le paludisme importé}

Ce travail a été réalisé à 2 échelles: échelle locale et échelle nationale. Léchelle locale a impliqué les hôpitaux régionaux qui recensent les cas de paludisme diagnostiqués dans leurs services. L'approche nationale a utilisé les données collectées par le CNREPIA auprès des hôpitaux du territoire français.

Lespèce plasmodiale dominante est $P$. falciparum avec un pic des cas en septembre (correspondant au retour des vacances) (DANIS et al., 2002 ; LEGROS et al., 2006). Les contaminations ont lieu majoritairement en Afrique de l'Ouest avec des variations régionales comme à l'hôpital de Marseille où l'on diagnostique des contaminations originaires principalement des Comores. Leur distribution spatiale est très hétérogène dans le Sud-Est de la France et les grandes agglomérations (Marseille, Nîmes et Montpellier) concentrent la majorité des cas.

La caractérisation de ces cas révèle que la majorité des personnes concernées sont des immigrés de première ou seconde génération retournant dans leur pays d'origine pour les vacances (LEGROS et al., 2006). L'étude de la population camarguaise permet donc d'identifier les communautés immigrées (de première ou seconde génération) et fournit donc une première approche de la distribution spatio-temporelle des parasites importés. Encore une fois, l'interdisciplinarité s'impose dans la mesure où des facteurs sociaux (dépendant eux-mêmes d'un contexte historique et économique) déterminent la répartition des parasites importés.

\section{Études des flux de population}

Parallèlement aux parasites importés donnant lieu à des cas cliniques, l'introduction de Plasmodium peut également être le fait de personnes porteuses chroniques, en provenance de zones endémiques. Il est donc nécessaire de répertorier et caractériser les flux de populations en provenance de zones endémiques aboutissant en Camargue. Dans ces flux, certains individus pourront correspondre à des porteurs chroniques. 
Létude préliminaire a révélé l'existence de 2 types de flux: des résidents camarguais se rendant en zones endémiques et revenant en Camargue (essentiellement touristes, professionnels et chasseurs/pêcheurs en safaris) et des résidents de zones endémiques de passage en Camargue (essentiellement touristes et travailleurs saisonniers). La majorité des touristes camarguais se rend plutôt en Afrique du Nord, zone où l'incidence du paludisme est quasi nulle. Les travailleurs saisonniers proviennent essentiellement du Maghreb, et les touristes étrangers de zones non endémiques. Finalement, ce travail a mis en évidence un risque faible d'introduction de parasites par des flux de personnes porteuses chroniques (BERTEMES et al., 2006).

L'introduction de Plasmodium dans des zones de transmission potentielle (identifiées à l'issue des 2 premières étapes) peut permettre d'initier un cycle de transmission autochtone. L'étude de la distribution spatio-temporelle des hommes porteurs de Plasmodium dans l'ensemble de la Camargue permettra donc d'évaluer le risque de ré-émergence du paludisme en Camargue. La caractérisation de ce risque passe, in fine, par le croisement de 3 distributions spatio-temporelles en lien avec le milieu (moustiques capables de transmettre des Plasmodium, humains disponibles pour les piqûres de ces moustiques et humains porteurs de Plasmodium) et l'identification de facteurs clés caractérisant les zones où ces 3 distributions se superposent.

\section{Conclusion}

Cette étude illustre la nécessité d'une approche interdisciplinaire afin d'évaluer le risque de ré-émergence de paludisme. La mise en commun des résultats de chacune des disciplines participantes permet d'aborder ce risque avec plus de réalisme. La biologie étudie les Anopheles et leur compétence vectorielle, l'anthropologie caractérise la population humaine au niveau local et les flux de populations humaines vers la Camargue, l'épidémiologie fournit des données concernant le paludisme importé en France, l'écologie permet d'aborder les questions relatives aux habitats des vecteurs, la géographie permet de spatialiser les données. De plus, l'approche 
interdisciplinaire souligne l'importance des facteurs historiques, culturels et socio-économiques qui conditionnent le risque vectoriel en Camargue, "contexte naturel fortement anthropisé». Ainsi, les changements dans l'utilisation du sol, l'urbanisation montante dans certaines zones de Camargue et le développement du tourisme et des loisirs influencent de manière importante les contacts possibles entre les humains et les Anopheles, en modifiant la densité et la répartition de ces deux derniers.

\section{Remerciements}

Nous remercions l'ensemble des personnes qui ont participé à ce travail dans le cadre des collaborations: Nicole Vernazza-Licht et Daniel Bley du CNRS, Marc-Éric Gruénais de l'IRD, Patrick Baudot, Vincent Bertemes, Céline Miglietti, Joanne Michelutti, Murielle Radix, Lucie Supiot de l'IUP ENTES, Annelise Tran du Cirad, Francis Schaffner, Christophe Lagneau, Grégory L'Ambert, Claire Duchet, Alexandre Carron, Charles Jeannin, Cécile Ivanes, Nicolas Sidos, Michel Babinot, Olivier Bardin, Didier Caire, Dominique Gindre et Nathalie Barras de l'EID-Méditerranée, Jérémiah Petit du syndicat mixte pour la protection et la gestion de la Camargue gardoise, Jean-Laurent Lucchesi, Nathalie Hecker, Grégoire Massez, Jean-Baptiste Nogues et Mathieu Chambouleyron de l'association les Amis du Vigueirat, Robert Meffre, Jean-Marie Egidio de la mairie de Mas Thibert, Didiet Basset du CHU de Montpellier, Laurence Lachaud de ]'hôpital de Nîmes, Muriel Roumier de l'hôpital d'Arles, Philippe Parola du CHU de Marseille, Fabrice Legros du CNREPIA, Cyrille Thomas du Centre français du riz, Thomas Balenghien et Florence Fouque de l'EPSP-TIMC/ENVL, Annie Walter de l'IRD.

Ce travail a été en partie financé par le ministère de l'Agriculture et le projet européen EDEN, GOCE-2003-010284 (cet article est référencé EDEN0167). Le contenu de cette publication est sous la seule responsabilité des auteurs et n'engage pas l'Union européenne.

\section{Références bibliographiques}

ARMENGAUd A., Legros F, QUATRESOUS l, BARRE H., VALAYER P, FANTON Y, D'ORTENZIO E., SCHAFFner F, 2006 - A case of autochthonous Plasmodium vivax malaria, Corsica. Euro Surveill. 11 : E061116 3.

Aznar J. C., Dervieux A., Grillas P., 2003 - Association between aquatic vegetation and landscape indicators of human pressure. Wetlands 23: 149-160. 
Baldari M., Tamburro A., Sabatinelli G., Romi R., Severini C., Cuccagna G., Fiorilli G., Allegri M. P., Buriani C., Toti M., 1998 Malaria in Maremma, Italy. Lancet 351 : 1246-1247.

Beier J. C., Perkins P. V., Wirtz R. A., Koros J., Diggs D., Gargan T. P. 2nd, KOECH D. K., 1988 - Bloodmeal identification by direct enzyme-linked immunosorbent assay (ELISA), tested on Anopheles (Diptera: Culicidae) in Kenya. J. Med. Entomol. $25: 9-16$.

Bertemes V., Miglietti C., Michelutti J., Radix M., Supiot L., 2006 Étude et caractérisation des flux de populations : risque d'introduction du paludisme en Camargue. IUP ENTES, université de Provence, Marseille.

Cuadros J., Calvente M. J., Benito A., Arevalo J., Calero M. A., SEgurA J., RuBio J. M., 2002 - Plasmodium ovale malaria acquired in central Spain. Emerg. Infect. Dis. 8 : 1506-8.

Danis M., Legros F., Thellier M., CAumes E., 2002 - Données actuelles sur le paludisme en France métropolitaine. Med. Trop., 62 : 214-8.

Dervieux A., Allard P., Naizor T., Auda Y., 2002 - « La Camargue est-elle un polder ? Changement et stabilité dans le delta du Rhône depuis le début du XIX siècle ». In : Équilibre et ruptures dans les écosystèmes durant les 20 derniers millenaires en Europe de l'Ouest, Besançon : 315329.

Detinova T. S, 1962 - Age-grouping methods in Diptera of medical importance with special reference to some vectors of malaria. Monogr. Ser. World Health Organ., $47:$ 13-191.

Doudier B., Bogreau H., Devries A., Ponçon N., Stauffer W., Fontenille D., Rogier C., Parola P., 2007 - Autochthonous malaria from Marseille to Minneapolis ? Emerg. Infect. Dis., 13 : 1236-38.

Kampen H., Proft J., Etti S., Maltezos E., Pagonaki M., Maier W. A., SEITZ H. M., 2003 - Individual cases of autochthonous malaria in Evros Province, northern Greece: entomological aspects. Parasitol. Res. 89 : 252-258.

Krüger A., Rech A., Su X. Z., TAnnich E., 2001 - Two cases of autochthonous Plasmodium falciparum malaria in Germany with evidence for local transmission by indigenous Anopheles plumbeus. Tropical medicine and international health, $6: 983-985$.

Legros F., Arnaud A., El Minouni B., Danis M., 2006 - Paludisme d'importation en France métropolitaine: données épidémiologiques 2001-2004. Bulletin épidémiologique hebdomadaire, 32 : 235-236.

MacDonald G., 1957 - The epidemiology and control of malaria. Oxford University Press, London. 
Poncon N., Toty C., Lambert G., Le Goff G., Brengues C., SCHAFFNeR F, FONTENILle D., 2007 - Biology and dynamics of potential malaria vectors in southern France. Malar. J., $6: 18$.

Proft J., Maier W. A., KAMPEN H., 1999 - Identification of six sibling species of the Anopheles maculipennis complex (Diptera: Culicidae) by a polymerase chain reaction assay. Parasitol. Res. 85 : 837-43.

SAUteT J., 1944 - À propos d'une épidémie de paludisme en Camargue. Mars méd. 2 : 53-64.

WHO 2007 - CISID-malaria. http://data.euro.who.int/cisid/malaria 



\section{Ruralité et asthme en France: retour d'expérience sur une approche interdisciplinaire}

\section{Nicole MATHIEU, Marie-Pierre ORYSZCZYN \\ Wandrille HUCY, Jean MACCARIO \\ Francine KAUFFMANN}

Alors que les recherches épidémiologiques en milieu africain se caractérisent depuis longtemps par des équipes interdisciplinaires où médecins et biologistes se mêlent aux anthropologues et géographes (RAYNAUT, 2001; LEGAY, 2006; et des références du colloque Milieux de vie et santé, SEH 2006) la recherche biomédicale française appliquée aux sociétés post-industrielles et aux milieux urbanisés fait assez rarement appel aux sciences sociales dans une conception élargie de l'interdisciplinarité. Lors des journées de l'association Natures Sciences Sociétés', le constat d'une dissociation, dans ce domaine, entre l'analyse sociologique - limitée à celle du système de santé ou aux réactions individuelles et collectives à une maladie (sida, cancer, maladies génétiques) d'une part, et une recherche médicale bien établie sur ses propres paradigmes disciplinaires d'autre part, avait déjà été souligné. On pourrait presque faire l'hypothèse qu'il existe deux modèles de recherche dans le domaine de la santé, l'un voisin de l'écologie 
humaine ${ }^{2}$ (médecine "environnementale » proche des populations pour lesquelles le problème se pose même si le recours aux méthodes statistiques est reconnu indispensable), l'autre où l'épidémiologie est liée structurellement à la statistique, l'enquête et le traitement des données étant considérés comme les fondements des résultats et de la démonstration, ce qui a tendance à minimiser si ce n'est à exclure la dimension individuelle et sociale du problème, et, de ce fait même, la pratique interdisciplinaire. Mais, compte tenu de la montée en puissance, dans nos sociétés techniques et mondialisées, de problèmes remettant en cause les interactions entre santé et environnement, - le rapport entre pollution de l'air, nuisances sonores et olfactives, sols contaminés par les industries, etc. et santé, voire même bien-être dans tous les lieux de vie des citadins (travail, circulation, habitat privé et collectif, de convivialité) -, n'est-il pas devenu urgent de subsumer ces distinctions d'école et de développer dans le champ santé/environnement l'humanisme et l'interdisciplinarité du premier modèle en complémentarité avec les méthodes éprouvées des grandes enquêtes biomédicales?

Tenter de répondre à cette interrogation en faisant un retour réflexif sur la recherche "Ruralité et asthme ${ }^{3}$ qui vient de s'achever (ORYSZCZYN et al., 2007) constitue l'enjeu de ce texte. En effet, à première vue, l'équipe de l'Inserm à l'origine de ce contrat et dont Francine Kauffmann (docteur en médecine, épidémiologiste et directrice de recherche à l'Inserm) est responsable, constitue un modèle classique de recherche liant épidémiologie et biostatistique. Spécialiste en pneumologie et immuno-allergologie avec comme thème de recherche «Épidémiologie des maladies respiratoires (asthme et broncho-pneumopathies obstructives chroniques), facteurs génétiques environnementaux », cette équipe est à la fois représentative et de la solidité du modèle français de recherche biomédicale, et d'une spécialité spécifique des milieux urbains où ces maladies respiratoires ne cessent de progresser. Comme dans la plupart des recherches sur la santé réalisées sur le territoire

2 Le titre du colloque SEH 2006 est sur ce point significatif faisant appel au concept de milieux de vie (voire de complexe pathogène dès l'ouverture) et aux pratiques interdisciplinaires

3 Réalisée dans le cadre de l'Action thématique concertée Environnement et Santé 2003. 
français, l'équipe s'appuie sur de grandes enquêtes fournissant des données individuelles comme l'enquête $\mathrm{E} \mathrm{N}^{4}$ portant sur 100000 femmes affiliées à la MGEN qui forment un échantillon suivi régulièrement et qui ont répondu à plusieurs dates à des questionnaires précis, détaillées et volumineux.

À l'origine, l'énoncé du problème "Ruralité et asthme" posé par les épidémiologistes se définit donc de façon relativement classique: "Recherche d'indicateurs rétrospectifs de ruralité (4000 communes) et application à l'étude épidémiologique du rôle protecteur des contacts avec les animaux de ferme dans l'asthme dans l'enquête PAARC (Pollution atmosphérique et affections respiratoires chroniques)». Il s'agit alors de valoriser une vaste enquête épidémiologique, initialement entreprise sur le thème de la pollution atmosphérique, pour tester l'hypothèse dite hygiéniste selon laquelle la diminution des contacts avec les agents infectieux dans la petite enfance pourrait expliquer l'augmentation de l'asthme et de l'allergie. Des contacts précoces avec le bétail peuvent représenter une source d'exposition aux agents infectieux.

Mais, différence sensible, "Ruralité et asthme" a pour codicille "Approche interdisciplinaire», ce qui traduit une intention d'élargissement méthodologique dont l'initiative revient à Francine Kauffmann. Car, même si la construction d'indicateurs reste le moyen central pour tester l'hypothèse, encore faut-il que les indicateurs - de ruralité - restituent le "milieu de vie" où les contacts précoces avec les animaux de ferme ont pu ou se sont produits. Il est alors nécessaire de faire appel à une discipline autre susceptible de discuter la question et d'apporter une solution au problème. Le pari de l'interdisciplinarité est engagé. Le récit de ce déplacement méthodologique sur une question mettant en relation « Milieux de vie et santé " et la réflexion sur ses résultats constituent donc l'enjeu de ce texte qui tente de donner une place égale aux points de vue des épidémiologistes comme à ceux des géographes engagés dans cette expérience d'interdisciplinarité.

4 Etude épidémiologique dirigée par Françoise Clavel-Chapelon, directrice de recherche à l'Inserm. Les auteurs remercient toute l'équipe E3N d'avoir permis l'accès à cette base de données. 
Dans un premier temps, nous reviendrons sur les questions justifiant la mise en place de l'interdisciplinarité des deux côtés disciplinaires.

Puis nous exposerons les "moments" de la co-construction de la recherche car la temporalité est un aspect essentiel de réflexion sur les démarches interdisciplinaires.

Enfin, nous présenterons les résultats que l'on peut attribuer à la pratique interdisciplinaire proprement dite.

En conclusion, nous tenterons d'évaluer les apports de l'interdisciplinarité non seulement du point de vue des épidémiologistes et des sciences sociales mais aussi dans la perspective générale esquissée dans l'introduction.

\section{De la nécessité d'une pratique interdisciplinaire: pourquoi et avec quels partenaires?}

Revenons d'abord aux questions de recherche qui se posent à l'équipe d'épidémiologie. Les objectifs y sont clairement cernés et il s'agit de: 1. Tester l'hypothèse du rôle protecteur des contacts précoces avec les bovins sur une population d'adultes; 2 . Valoriser l'enquête PAARC soit 18000 sujets recrutés dans 7 villes de France, tous nés avant 1950, et dont le lieu de naissance est noté dans le questionnaire; 3 . Construire un indicateur simple permettant une estimation rétrospective du contact potentiel avec le bétail ; 4. Valider l'indicateur écologique construit en testant sa cohérence avec des données individuelles recueillies dans 2 autres enquêtes, les enquêtes EGEA ${ }^{5}$ et E3N pour pouvoir l'étendre aux recherches épidémiologiques qui tentent d'intégrer les questions d'environnement.

Mais sur quel concept l'estimation rétrospective du contact potentiel avec le bétail peut-elle s'appuyer? Le terme de "rural» est pour les épidémiologistes, en première approximation, une piste pour 
bâtir cet «indicateur simple et rétrospectif». Mais alors quels critères permettent de définir la "ruralité " (le milieu rural) sur laquelle repose l'hypothèse? Comment passer de la commune de naissance au milieu rural? Comment construire un indicateur statistique qui ait une fiabilité et statistique et "géographique " puisqu'il s'agit d'identifier des lieux et milieux avec des propriétés spécifiques?

Du côté des épidémiologistes, c'est à ce point du questionnement que le recours à un partenaire de sciences sociales spécialiste de la «ruralité» se révèle comme un besoin, une nécessité pour mener la recherche jusqu'au résultat concluant.

Mais la demande est encore vague et ne définit clairement ni une discipline au sein des «études rurales» (sociologie? géographie? agronomie? économie?...), ni une compétence thématique ou d'expertise. De bouche à oreille et par la fréquentation des rencontres interdisciplinaires organisées par le ministère de la Recherche, le choix se porte sur l'équipe du Ladyss connue à la fois pour son Observatoire des relations rural/urbain (MATHIEU, ROBERT, 1998) et pour son engagement dans les pratiques interdisciplinaires (Jollivet, 1992) et la revue Natures Sciences Sociétés.

Du côté des chercheurs du Ladyss, la proposition de partenariat rencontre un accueil naturellement favorable dans la mesure où elle appelle un déplacement méthodologique sur une question mettant en relation "milieux de vie et santé".

D'abord, toute occasion de clarifier l'usage des notions de rural et d'urbain en les confrontant à une thématique nouvelle s'inscrit dans l'ambition de l'Observatoire (MATHIEU, 2002). De plus, avec cette question, la clarification se doit d'approfondir ce qui distingue campagne, espace rural et milieu rural. La réflexion sur les notions de «lieux» et "milieux» est d'ailleurs une préoccupation centrale de plusieurs chercheurs géographes du Ladyss. S'appuyant sur la pensée de Max Sorre (genre de vie, complexe pathogène) et d'Éric Dardel, l'équipe tente depuis plusieurs années de souligner la valeur heuristique des concepts de milieu, de mode d'habiter et d'habitants dans leurs rapports avec ceux de ruralité et d'urbanité (MATHIEU, 2007). Avec l'irruption de l'utopie du développement durable (JOLllvet, 2001) la question de l'habitabilité des lieux et de la gestion durable des milieux de 
vie constitue un axe majeur des recherches en particulier sur la ville (ROBIC et al., 2001 ; MATHIEU, GUERMOND, 2005; HuCY et al., 2005).

Enfin l'expérimentation de pratiques interdisciplinaires entre sciences sociales et sciences de la vie autour du problème du «bien-être » (MATHIEU et al. 1997; LEGAY, 2004) y est considérée comme un enjeu et le fait de travailler avec des épidémiologistes une occasion de plus de tester la «méthode d'assemblage» de disciplines éloignées comme moyen de mener un problème jusqu'aux résultats (JOLLIVET, LEGAY, 2005).

En somme, de part et d'autre le besoin d'interdisciplinarité est reconnu: il est tiré par la question des épidémiologistes, celle-ci est acceptée par les géographes pour la potentialité de pratique interdisciplinaire qu'elle offre. Il s'agit alors de co-construire une démarche dont l'aboutissement - tester les hypothèses et les démontrer - est l'objectif. Démarche longue dont la progression est marquée par différents temps: le temps de l'approche où se jouent parallèlement et la reconnaissance de l'identité et des compétences de l'autre discipline, et l'assurance que le problème énoncé est compris de façon équivalente par les partenaires; le temps du choix des protocoles et des dispositifs marqué souvent par des conflits de méthode avant d'aboutir à des accords voire un consensus; le temps de la validation des résultats qui pour les épidémiologistes est une obligation.

\section{Le premier temps de la marche}

Le premier temps de la mise en œuvre de la pratique interdisciplinaire est un moment d'apprentissage - on pourrait dire d'apprivoisement - qui s'organise autour du problème complexe impliquant des savoirs autres que ceux de l'épidémiologie. Il se déroule sous le signe de la curiosité, de l'attrait pour l'autre, d'un certain désir de découverte.

Se pose d'abord la question du langage et du sens des mots autour desquels se formule le problème. "Vous avez dit rural ? " 
mais qu'est ce que la ruralité ? Ce mot, qui paraît d'autant plus facile à comprendre qu'il fait partie du langage courant, se révèle vite porteur de malentendus, voire de contresens. Les épidémiologistes pensent, au départ, que ce qu'on appelle «commune rurale " répond à leurs attentes. Les géographes leur expliquent ce que recouvrent la notion statistique et les controverses auxquelles elle a donné lieu. Ce sont des données essentiellement démographiques (taille de la population, présence de migrations alternantes et proximité des villes) et historico-administratives (grandes différences de surface, de systèmes de peuplement...). Bien que dites "objectives", elles véhiculent une conception dominante des représentations sociales de la ruralité et des relations villes/campagnes qui les marquent et les éloignent, aujourd'hui, de la relation à l'agriculture, aux systèmes agraires et à la société paysanne ou rurale (MATHIEU, 1990, 1998).

Les épidémiologistes persistent dans leur demande: la ruralité dont vous devez nous donner l'identité statistique est un «lieu» (de naissance) où les individus dont nous avons les caractéristiques de santé (asthme et allergies) ont été en contact avec des bovins. Autrement dit, répondent les géographes, des lieux où les systèmes d'élevage sont dominants. Or, la «commune rurale» (comme lieu de naissance) est une notion statistique qui est très éloignée de la probabilité d'avoir vécu dans une ferme ou à proximité d'élevages bovins traditionnels où les contacts avec les animaux et la consommation de lait cru sont quasi quotidiens. Ce que vous demandez - la présence d'un lieu dans une région d'élevage - se trouve plutôt dans la notion elle-même statistique de "région agricole» qui est d'ailleurs une des bases des recensements de l'agriculture. Certes, répondent les épidémiologistes mais pour être valide notre recherche doit pouvoir confronter les données dans différentes ruralités: des ruralités «d'élevage» et des ruralités où les contacts précoces avec les bovins - comme ce qui se passe vraisemblablement pour les milieux urbains - ont une probabilité quasi nulle de se produire.

Après un patient et long échange, documents à l'appui, les malentendus sont levés et l'objectif à atteindre - nous le verrons plus loin dans les résultats - est bien d'identifier et de classer des types de communes rurales selon leur propension à juxtaposer des habitants et des animaux de ferme. 
Dans ce premier temps de la recherche la curiosité et l'appétit de connaissance envers l'autre discipline sont essentiels. Du côté des sciences sociales, on assiste avec intérêt aux séminaires où l'équipe d'épidémiologie invite des équipes étrangères travaillant sur le même problème à confronter leurs méthodes et leurs résultats et, en séance interne de travail, on se familiarise avec les enquêtes, leur ampleur et leurs questionnaires très différents où parfois sont pris en compte des indicateurs du milieu de vie ou de l'alimentation (par exemple dans E3N). Inversement, les épidémiologistes manifestent - intuitivement - le besoin d'en savoir plus sur le concept de mode d'habiter dans sa relation avec le rural et l'urbain parce qu'il pourrait être heuristique. Un séminaire est organisé à Villejuif tout entier consacré à l'explicitation de ce concept en présence de tous les membres de l'équipe. Les questions posées après l'exposé sont remarquables: quelle est la genèse du concept et son rapport avec le problème concret posé? Comment les sciences sociales démontrent et quel est leur usage des statistiques? Cette attention réciproque, cette curiosité pour les fondements et l'invention théoriques d'une discipline, cette volonté de comprendre suffisamment pour ne pas instrumentaliser l'autre, constituent les ingrédients indispensables pour franchir ce premier seuil de la démarche interdisciplinaire. Apprendre à se connaître en multipliant les rencontres, les séminaires, les essais requiert un dialogue relativement long. Ce premier temps du processus d'apprentissage permet de préciser les besoins et les compétences scientifiques, d'évaluer les affinités et surtout les possibilités de "pratiquer", de mettre en pratique cette «méthode d'assemblage» entre sciences de la nature et sciences sociales qu'est l'interdisciplinarité (Jollivet, Legay, 2005).

\section{Le temps difficile: choc des cultures scientifiques et des savoir-faire}

Mais une fois passé ce temps initial du partage des questions et lorsqu'il s'agit de mettre la démarche en œuvre autour d'une coconstruction des dispositifs et du travail commun, alors surgissent 
les premières vraies difficultés. Une période s'ouvre où les différences, voire les oppositions portent sur les pratiques scientifiques elles-mêmes (méthodes, techniques de travail, espaces de publication ${ }^{7}$ ) entraînant des incompréhensions qui dépassent celles portant sur les concepts ou sur l'énoncé du problème accepté de part et d'autre.

En somme, dans la phase de répartition du travail à exécuter pour l'objectif commun, le processus d'apprentissage et la négociation de l'assemblage se compliquent.

Tout d'abord, comme nous l'avons dit en introduction, les épidémiologistes des pays "développés" ne conçoivent pas d'autres moyens de démonstration de leurs hypothèses que les méthodes statistiques. Cela au point que, pour qu'il n'y ait pas biais statistique, la construction des indicateurs de milieu qui leur semble du ressort des géographes, doit se faire «en aveugle" c'est-à-dire sans connaître les données médicales proprement dites. Lorsque les sciences sociales proposent de répondre au problème posé par l'étude de cas - celle des monographies de communes représentant des milieux ou celle des trajectoires individuelles de lieux de vie représentant la diversité des rapports des individus aux milieux le scepticisme est évident. L'important est de remplir les matrices avec des données fiables. À plusieurs reprises, le terme de "à dire d'expert» est avancé pour obtenir des partenaires les réponses attendues, quantitatives ou qualitatives, mais toujours à coder et à introduire dans une matrice. Or, c'est le problème posé qui intéresse les sciences sociales et le terme d'expert, de leur point de vue, les confine dans une posture - l'expertise - qui est en contradiction avec celle de l'engagement dans la démarche heuristique de l'interdisciplinarité. Les savoir-faire sont bousculés et il faut accepter que les compétences demandées ne soient pas celles que l'on pensait pouvoir apporter dans la recherche. Pour les «seniors» la chose est acceptable car l'expérience d'un «déplacement» méthodologique est toujours tentante. Mais la difficulté s'accroît quand il s'agit des jeunes chercheurs: doit-on prendre le risque de

7 Lorsqu'il s'agit de présenter des résultats en épidémiologie et biostatistique à I'Inserm, on se doit de publier dans des revues internationales de langue anglaise (ORYSZCZYN et al., 2003; 2005; KAUFFMANN et al., 2002), ce qui n'est pas la règle pour les chercheurs de sciences sociales. 
les impliquer dans ce «terrain d'aventure» où aucune compétence n'est prédéfinie, où le bricolage et le tâtonnement sont la règle, où, enfin, la validation est à inventer et à négocier entre des disciplines très éloignées l'une de l'autre?

\section{Le temps des pratiques au service des résultats}

Dans le vécu des chercheurs engagés dans cette «approche interdisciplinaire", cette période, aussi longue que la précédente, se divise - pour des raisons de méthode statistique -en deux moments distincts: celui de la construction d'un indicateur dans laquelle le rôle des géographes a été très important dans une pratique interdisciplinaire équilibrée; celui de la validation que les épidémiologistes ont mené seules sans pour autant tirer la couverture à elles du fait de la très grande interdépendance - à leurs yeux - entre la conduite de la première étape et la qualité de l'indicateur construit et la deuxième à savoir la validation des hypothèses de départ.

\section{La construction d'un indicateur de «ruralité »: une étroite collaboration}

Après avoir convenu que l'indicateur de ruralité recherché se définissait au croisement du Recensement général de la population (RGP) (commune rurale, zone à dominante rurale) et du Recensement général de l'agriculture (RGA), le travail interdisciplinaire s'est organisé autour de questions à résoudre les unes après les autres: choix de la période de référence (1955 ou 1970), choix de l'unité géographique pertinente, définition de classes simples pour les indicateurs, validation de l'indicateur. Dans la mesure où le but à atteindre était essentiellement expérimental et méthodologique - définir un mode de construction des indicateurs -, les travaux ont été réalisés sur deux régions tests, l'Aquitaine et la Haute-Normandie en raison de leur contraste connu en ce qui concerne leurs systèmes de production agricole dominants, la première avec OTE (Orientation technique des exploitations) céréalière et la seconde avec l'élevage. 
Il fallait d'abord faire le choix de la période de référence, les sujets de l'enquête PAARC étant tous nés avant 1950. La date du RGP ne posait pas de problème: celui de 1962 était recevable pour représenter le milieu de vie "humain » des sujets de l'enquête. En revanche, en ce qui concerne les RGA, il y avait deux possibilités, les RGA 1955 et 1970, dont il fallait évaluer les avantages et les inconvénients avant de construire une des bases du dispositif. Certes, le RGA le plus approprié était celui de 1955 mais ce dernier était non informatisé - le premier RGA informatisé date de 1970 - et les données utilisées devaient être extraites manuellement, ce qui représentait un gros travail.

Pour déterminer si le RGA de 1970 pouvait être utilisé pour des sujets, tous nés avant 1950 et aussi pour essayer de répondre au problème des nombreuses données manquantes ( secret statistique » portant sur les exploitations quand leur nombre est inférieur au seuil de 3 dans les communes unités de base du recensement), il a été décidé de comparer l'évolution d'indicateurs entre 1955 et 1970 basés sur les données de recensement dans 2 régions contrastées. La Haute-Normandie a beaucoup évolué entre 1946 et 1970 en raison d'une conversion en herbe de ces régions qui sont devenues des pôles d'élevage. Dans cette région, le nombre de communes avec bovins n'a pas augmenté, mais le cheptel s'est agrandi. La région Haute-Normandie comprend 1219 communes rurales, 87 cantons et 19 petites régions agricoles (PRA) et concerne 400 communes dans l'enquête PAARC. L'Aquitaine est une région qui comprend beaucoup de systèmes agricoles (vignobles, céréales, polyculture dans les vallées). Certaines communes n'ont pas de vaches sur leur sol, d'autres ont des vaches à viande avec peu de production de lait (la Blonde d'Aquitaine). La région Aquitaine comprend 2009 communes rurales, 207 cantons et 68 PRA et concerne 486 communes dans l'enquête PAARC.

Un travail important de recueil de données concernant le bétail sur le RGA de 1955, non informatisé, a été fait pour les deux régions. Les renseignements sur le bétail (bovins, équins, ovins, porcins, nombre d'exploitations agricoles et surface totale) pour chaque commune ont été relevés aux archives nationales et au ministère de l'Agriculture. Les changements intervenus entre les numéros des communes en 1955 et 1970 ont dû être faits manuellement. Des renseignements étaient disponibles pour 
Tableau I.

Nombre de communes par département selon les recensements

\begin{tabular}{|lcc|}
\hline & $\begin{array}{c}\text { RGA 1955 } \\
\text { (n = 852) }\end{array}$ & $\begin{array}{c}\text { RGA 1970 } \\
\text { (n = 886) }\end{array}$ \\
\hline Aquitaine & & \\
Dordogne (24) & 71 & 75 \\
Gironde (33) & 214 & 221 \\
Landes (40) & 53 & 60 \\
Lot-et-Garonne (47) & 55 & 59 \\
Pyrénées-Atlantiques (64) & 69 & 71 \\
\hline Haute-Normandie & & \\
Eure (27) & 106 & 107 \\
Seine-Maritime (76) & 284 & 293 \\
\hline
\end{tabular}

852 communes (soit 3,8\% de communes manquantes par rapport au RGA 1970). Malheureusement, une variable essentielle, le nombre d'habitants ne figurait pas dans ces données. Le tableau 1 présente le nombre de communes par département pour les 2 recensements.

Les sujets de l'enquête PAARC avaient été recrutés dans 7 villes de France (dont Rouen et Bordeaux). Le tableau 2 indique la répartition, dans l'enquête, des communes au sein des départements pour les 2 régions considérées. Les 2 départements les plus concernés sont - comme attendu -, la Gironde et la Seine-Maritime. La région de Haute-Normandie a une surface plus petite que l'Aquitaine, selon les 2 RGA. La surface agricole pour la Haute-Normandie est 1,51 fois plus élevée en 1970 par rapport à 1955 , et pour l'Aquitaine de 2,57 (tabl. 2).

L'analyse cartographique et la comparaison des résultats localisés en 1955 et 1970 ont compté dans l'évaluation de la meilleure solution à prendre.

Les cartographies des communes de la Haute-Normandie et l'Aquitaine, réalisées par Wandrille Hucy, privilégiaient les indicateurs permettant de montrer un gradient d'intensivité de l'élevage bovin et notamment laitier et la présence de petites structures d'exploitation d'élevage (cf. cartes par commune et PRA en 1955 et en 1970 in ORYSZCZYN et al., 2007). Il est clair que les 
Tableau 2.

Élevage en Haute-Normandie et Aquitaine suivant les données des RGA de 1955 et 1970

\begin{tabular}{|c|c|c|c|c|c|c|}
\hline & \multicolumn{3}{|c|}{1955} & \multicolumn{3}{|c|}{1970} \\
\hline & 2 régions & Haute-Normandie & Aquitaine & 2 régions & Haute-Normandie & Aquitaine \\
\hline Bovins $m \pm 5$ & $\begin{array}{c}507,9 \pm 401,1 \\
(n=746)\end{array}$ & $\begin{array}{c}610,9 \pm 378,4 \\
(n=347)\end{array}$ & $\begin{array}{c}418,3 \pm 399,2 \\
(n=399)\end{array}$ & $717,1 \pm 580,7$ & $912,4 \pm 573,0$ & $547,1 \pm 532,4$ \\
\hline Bovins/ST, $m \pm$ & $0,63 \pm 0,45$ & $0,86 \pm 0,26$ & $0,43 \pm 0,48$ & $0,61 \pm 0,50$ & $0,99 \pm 0,48$ & $0,28 \pm 0,19$ \\
\hline Équidés $m \pm s$ & $\begin{array}{c}39,8 \pm 36,0 \\
(n=474)\end{array}$ & $\begin{array}{c}41,2 \pm 27,7 \\
(n=199)\end{array}$ & $\begin{array}{c}38,8 \pm 41,0 \\
(\mathrm{n}=275)\end{array}$ & $17,4 \pm 29,6$ & $8,5 \pm 12,7$ & $23,8 \pm 36,0$ \\
\hline Équidés/ST, $m \pm$ & $0,15 \pm 2,11$ & $0,06 \pm 0,03$ & $0,21 \pm 2,77$ & $0,009 \pm 0,011$ & $0,008 \pm 0,011$ & $0,011 \pm 0,246$ \\
\hline Ovins $m \pm$ & $\begin{array}{c}223,8 \pm 602,3 \\
(n=546)\end{array}$ & $\begin{array}{c}120,4 \pm 211,3 \\
(n=274)\end{array}$ & $\begin{array}{c}326,9 \pm 814,2 \\
(n=272)\end{array}$ & $310,4 \pm 885,9$ & $106,9 \pm 185,7$ & $515,3 \pm 1208,2$ \\
\hline Ovins/ST, $\mathrm{m} \pm$ & $0,22 \pm 0,49$ & $0,16 \pm 0,26$ & $0,29 \pm 0,64$ & $0,16 \pm 0,34$ & $0,11 \pm 0,21$ & $0,21 \pm 0,43$ \\
\hline Porcins, $\mathrm{m} \pm \mathrm{s}$ & $\begin{array}{c}145,0 \pm 171,7 \\
(n=606)\end{array}$ & $\begin{array}{c}143,2 \pm 158,5 \\
(n=239)\end{array}$ & $\begin{array}{c}146,2 \pm 179,9 \\
(n=367)\end{array}$ & $213,8 \pm 316,8$ & $175,4 \pm 282,9$ & $238,8 \pm 335,0$ \\
\hline Porcins $/ S T, m \pm s$ & $0,19 \pm 0,46$ & $0,27 \pm 0,70$ & $0,14 \pm 0,15$ & $0,16 \pm 0,40$ & $0,20 \pm 0,59$ & $0,13 \pm 0,18$ \\
\hline Exploitations, $\mathrm{m} \pm \mathrm{s}$ & $\begin{array}{c}79,9 \pm 78,4 \\
(n=838)\end{array}$ & $\begin{array}{c}37,9 \pm 23,5 \\
(n=379)\end{array}$ & $\begin{array}{c}114,6 \pm 90,0 \\
(n=459)\end{array}$ & $56,3 \pm 54,4$ & $31,6 \pm 19,8$ & $76,7 \pm 64,5$ \\
\hline$S T, m \pm s$ & $\begin{array}{c}853,16 \pm 674,9 \\
(n=852)\end{array}$ & $\begin{array}{c}667,9 \pm 400,5 \\
(n=390)\end{array}$ & $\begin{array}{c}1009,6 \pm 807,4 \\
(n=462)\end{array}$ & $1870,7 \pm 2316$ & $1007,7 \pm 656,3$ & $2599,2 \pm 2895,5$ \\
\hline
\end{tabular}

$\mathrm{n}=$ le nombre de communes ayant les informations pour les 2 RGA, m: moyenne, $\mathrm{s}$ : écart-type, ST = Surface totale. Les diminutions sont représentées en italique. 
communes de la Haute-Normandie ont, comme attendu, beaucoup plus de bovins que l'Aquitaine. La comparaison des régions a été réalisée en considérant les deux indicateurs densité de bovins par communes et le nombre de bovins par exploitation. Lanalyse de ces cartes montre que le gradient observé en 1970 reflétait assez bien la situation du RGA de 1955. Au terme de cette phase pratique menée en constante interaction entre les chercheurs, il a été conclu qu'il était possible de se servir pour la France entière des données de 1970 qui étaient informatisées.

Une fois la base du RGA de 1970 admise, les questions du choix de l'unité géographique pertinente et l'évaluation cartographique de divers indicateurs se sont posées et constituent les étapes suivantes de la démarche.

Rappelons l'objectif qui était de mettre en relation un paramètre de santé et un paramètre d'exposition. Le premier indicateur (classique) retenu pour distinguer le milieu rural du milieu urbain est le fait d'habiter une commune de moins de 5000 habitants $^{8}$.

Le premier indicateur d'exposition choisi a été le nombre de bovins par habitants $(\mathrm{B} / \mathrm{H})$ dans les communes rurales, c'est-à-dire dans les communes de moins de 5000 habitants, l'hypothèse était que plus le ratio augmentait et plus la probabilité d'être en contact avec une vache était forte.

Quant à la région, elle peut être décomposée en zones géographiques plus ou moins grandes: les Petites régions agricoles (PRA), les cantons et les communes. En France, il y a 36600 communes, 3644 cantons, 714 petites régions agricoles. Le RGA ne donnait pas directement les données en fonction des PRA et celles-ci ont été reconstituées secondairement.

Les premiers essais de calcul dans une zone géographique ont montré que le regroupement des communes était nécessaire. Si la commune était choisie comme unité d'exposition, certaines communes se retrouvaient avec peu de sujets. Afin de déterminer l'unité géographique la plus pertinente, le ratio (nombre de

8 Ce n'est pas celui des moins de 2000 habitants agglomérés au chef-lieu de la commune qui est toujours la base de la définition statistique des communes rurales en France mais - tout en restant démographique - un critère qui correspond à une définition de l'OCDE et de l'Union européenne. 
bovins/habitants) a été construit au niveau de la commune, du canton et au niveau de la PRA en réunissant les communes rurales au sein de ces unités pour les deux dernières unités. La question à résoudre devenait alors: quelle unité géographique pertinente choisir: la commune, le canton ou la PRA ?

La définition de l'unité géographique dépend: 1) de la pertinence par rapport à l'exposition; 2) surtout des données disponibles avec lesquelles on pourrait croiser cette caractérisation géographique:

- La commune n'est pas toujours appropriée car certaines d'entre elles comprennent peu d'habitants et, étudier ensuite un paramètre de santé devient difficile, comme indiqué plus haut.

- Le canton est une unité administrative datant de la Révolution. De forme et de taille régulière (un polygone régulier autour d'un centre; le chef-lieu de canton) et de taille équivalente, il regroupe un certain nombre de communes, ensemble souvent représentatif d'un certain type de production (céréalier, viticole, élevage...).

- Le découpage en PRA date des années 1950 et reprend l'idée du découpage de la France en "pays". Ces unités géographiques ont été définies en collaboration entre géographes et agronomes sur la base d'une spécificité de milieu (sols et climat) à laquelle étaient liés des systèmes de production agricole et des paysages. Les PRA incluent un nombre variable de cantons; l'unité PRA est donc supérieure à l'unité canton.

Dans les deux régions étudiées en détail, il y avait 3319 communes, dont 3228 étaient considérées comme rurales. Elles étaient agrégées en 294 cantons et 87 PRA.

Des cartographies ont été de nouveau réalisées pour montrer les PRA en Normandie et en Aquitaine et la dispersion des indicateurs étudiée par des modèles statistiques dits emboîtés [commune, canton, région] et [commune, PRA et région]. Comme attendu, la dispersion est moindre à l'intérieur d'un canton par rapport à la PRA. En conclusion, il est apparu que les PRA étaient de taille trop importante, et que les considérer masquait une hétérogénéité d'intérêt. Il a donc été conclu de prendre le canton comme unité.

Au quasi terme de cette étape s'est posé le cas des communes de plus de 1000 habitants. Afin d'appréhender au mieux l'exposition, 
il est ensuite apparu que considérer toutes les communes regroupées dans leur canton représentait une perte d'information et au total l'indicateur a été basé sur les données de la commune quand celle-ci avait plus de 1000 habitants et au niveau du canton quand la commune avait moins de 1000 habitants (en regroupant alors toutes les communes du canton de moins de 1000 habitants). Au total, l'indicateur retenu de "ruralité " (contact avec les bovins), décrit dans le tableau 3 prend en compte le nombre d'habitants (critère classique pour définir les communes non rurales), le rapport nombre de bovins/habitants pour les autres communes avec, pour les communes de moins de 1000 habitants, la proportion dans le canton (optimisation pour les données manquantes et la variabilité intercommunes d'un même canton). Suivant le même principe, des indicateurs peuvent être construits pour d'autres animaux. Le tableau 4 montre l'application de l'indicateur dans l'enquête PAARC.

Tableau 3.

Construction de l'indicateur de ruralité $\mathrm{B} / \mathrm{H}$ en $\mathbf{5}$ classes (qui peut être réduit à 3 si population plus réduite)

\begin{tabular}{|c|c|c|c|}
\hline \multirow[b]{2}{*}{ Bovins/habitants } & \multicolumn{3}{|c|}{ Nombre d'habitants de la commune } \\
\hline & $\geq 5000$ & $1000-4999$ & $<1000$ \\
\hline Quel que soit B/H & 0 & & \\
\hline $\mathrm{B} / \mathrm{H}<1$ dans la commune & & 1 & \\
\hline $1,0 \leq \mathrm{B} / \mathrm{H}<2,0$ dans la commune & & 2 & \\
\hline $2,0 \leq \mathrm{B} / \mathrm{H}<2,5$ dans la commune & & 3 & \\
\hline $2,5 \leq \mathrm{B} / \mathrm{H}<3,0$ dans la commune & & 4 & \\
\hline $\mathrm{B} / \mathrm{H} \geq 3,0$ dans la commune & & 5 & \\
\hline $\mathrm{B} / \mathrm{H}<1$ dans le canton* & & & 1 \\
\hline $1,0 \leq \mathrm{B} / \mathrm{H}<2,0$ dans le canton & & & 2 \\
\hline $2,0 \leq \mathrm{B} / \mathrm{H}<2,5$ dans le canton & & & 3 \\
\hline $2,5 \leq \mathrm{B} / \mathrm{H}<3,0$ dans le canton & & & 4 \\
\hline $\mathrm{B} / \mathrm{H} \geq 3,0$ dans le canton & & & 5 \\
\hline
\end{tabular}

* En regroupant les communes de moins de 1000 habitants du canton. 
Tableau 4.

Application de l'indicateur dans l'enquête épidémiologique

(18000 sujets) pour la commune de naissance ( 4000 communes)

\begin{tabular}{|ccc|}
\hline $\begin{array}{c}\text { Indicateur de ruralité } \\
\text { contact avec les bovins }\end{array}$ & $\begin{array}{c}\text { Nombre } \\
\text { de sujets }\end{array}$ & $\begin{array}{c}\text { Pourcentage } \\
\text { de sujets }\end{array}$ \\
\hline 0 & 13261 & 74,9 \\
1 & 2785 & 15,7 \\
2 & 904 & 5,1 \\
3 & 286 & 1,6 \\
4 & 198 & 1,1 \\
5 & 281 & 1,6 \\
\hline
\end{tabular}

\section{Validation et relation de l'indicateur avec l'asthme: des temps exclusivement disciplinaires}

Dans cette phase finale de la recherche qui se décompose en deux étapes distinctes, il faut reconnaître que les géographes ne sont plus que les témoins de la logique démonstrative des épidémiologistes et ne participent plus qu'à la satisfaction quand les résultats sont probants. Le succès - disent les épidémiologistes - doit être partagé car il repose sur la qualité interdisciplinaire du travail des étapes précédentes. Les résultats ont été présentés à des congrès internationaux de pneumologie (ORYSZCZYN et al., 2003, 2005) et sont brièvement décrits ci-dessous. La faisabilité et la validation des hypothèses reposent sur l'utilisation et le croisement des données d'enquêtes successives. L'enquête EGEA a mis en évidence la relation des paramètres allergiques avec la «vie à la campagne» (KAUFFMANN et al., 2002). L'étude E3N a permis de passer de la faisabilité à la validation car des données avaient été directement recueillies auprès des femmes lors d'un suivi sur leurs contacts avec des animaux de ferme ${ }^{9}$.

9 II est à souligner que ces étapes n'ont pu être possibles que par l'obtention d'autres contrats sur cette thématique de recherche initiée à l'aide du présent contrat (en particulier un contrat de l'AFSSE intitulé "Rôle des contacts avec les animaux de ferme dans l'asthme: I'enquête E3N » et un contrat ANR SEST (programmes Sante/Environnement et Santé/Travail) 2005 : «EGEA: Facteurs environnementaux et interactions gène environnement dans l'asthme et l'allergie $\%$. 
Les adultes de l'étude EGEA avaient été interrogés sur le fait d'avoir vécu à la campagne dans leur vie et en cas de réponse positive l'âge de début était demandé. Il était ainsi possible de définir une exposition précoce (avoir vécu à la campagne avant l'âge d'un an). Il apparaît que $18,8 \%$ des habitants des communes de plus de 5000 habitants déclaraient avoir vécu à la campagne avant l'âge d'un an alors que les sujets habitant dans des communes de moins de 5000 habitants étaient plus nombreux à le déclarer (différence statistiquement significative). Ce résultat était cohérent avec la définition de ruralité selon les spécialistes du monde rural, pour les sujets interviewés la campagne équivaut à zone rurale. Aucun gradient n'était observé pour les 3 classes de ruralité situées dans les communes rurales. Les résultats étaient voisins quand on considérait la variable «avoir vécu à la campagne dans sa vie» (ORYSZCZYN et al., 2003).

L'enquête E3N comprenait 50 fois plus de sujets que la précédente et permettait une réelle validation de l'indicateur. $37,3 \%$ des femmes étaient nées dans une zone rurale, dont 2,3\% dans la classe la plus élevée de l'indicateur de ruralité. Dans cette enquête, nous avions une information explicite sur la vie à la ferme trois mois de suite dans l'enfance pour 68483 femmes. Plus le gradient de ruralité augmentait et plus le pourcentage de sujets ayant vécu à la ferme dans l'enfance augmentait (14,4\%, 29,8\%, 46,7\%, 50,8\%, 53,8\%, $53,9 \%$ ) (fig. 1). Il n'y avait pas de différence entre les deux classes les plus élevées.

Figure 1.

Dans l'enquête E3N (68483 femmes), nombre de sujets déclarant avoir vécu dans une ferme au moins 3 mois de suite.

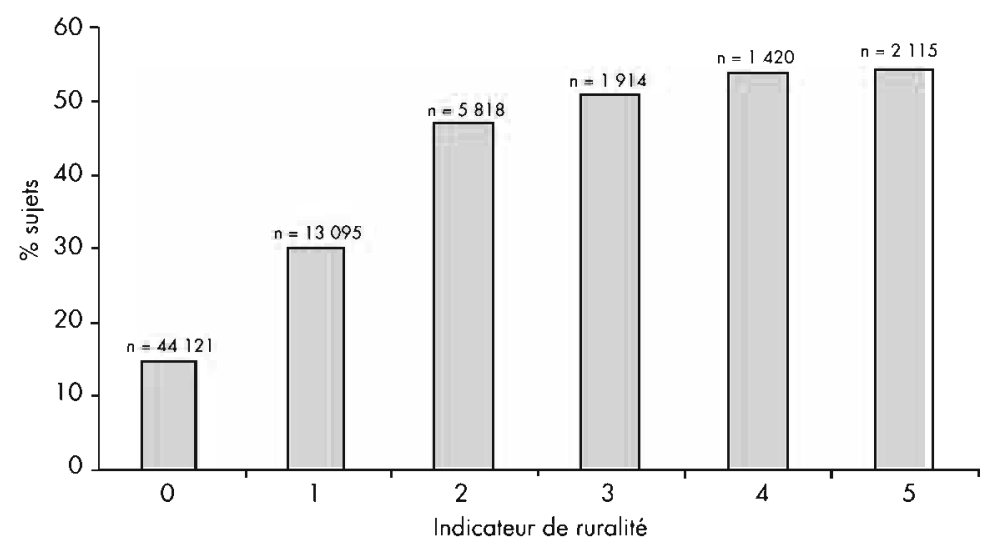


Les femmes ayant vécu dans une ferme au moins 3 mois de suite ainsi que les femmes ayant vécu à la ferme dans leur enfance ont ensuite été étudiées plus particulièrement (ORYSZCZYN et al., 2005). L'ensemble des résultats montre que l'indicateur a donc une excellente sensibilité pour détecter des sujets réellement très "exposés" puisque plus de $50 \%$ des femmes dans les classes les plus élevées de l'indicateur avaient rapporté avoir vécu dans une ferme. Par ailleurs, les résultats confirment dans cette population que dans la catégorie classique de communes non rurales, définie par le nombre d'habitants de la commune, l'indicateur a une bonne spécificité puisque moins de $5 \%$ de sujets déclaraient avoir eu des parents fermiers.

$\mathrm{Au}$ total, l'indicateur proposé s'avère très lié aux réponses aux questions directes sur la vie à la campagne, à la ferme, aux contacts avec les animaux de ferme, avec peu de différences en ce qui concerne les 3 catégories les plus élevées. Ces catégories s'avèrent très difficiles à distinguer dans des études sur des effectifs modestes (comme EGEA) et sont peu discriminantes dans des études sur des effectifs importants. On peut conclure que l'indicateur en 4 classes est le plus approprié à être généralisé dans d'autres études.

\section{Relation de l'indicateur de ruralité avec l'asthme Application dans l'enquête PAARC}

L'étude a porté sur 17647 sujets adultes de l'enquête PAARC réalisée en 1975. Dans l'analyse a été considéré l'asthme au cours de la vie défini par la réponse positive à l'une des questions «Avezvous des crises d'essoufflement au repos avec des sifflements dans la poitrine?" ou "Avez-vous eu des crises d'asthme". Parmi les sujets ayant déclaré «des crises d'asthme» (diagnostic d'asthme dans le tableau 5), l'analyse a porté sur l'asthme de l'enfance défini par la survenue de la première crise avant l'âge de 16 ans. Enfin, un indicateur potentiel d'allergie a été défini avec les données disponibles, en considérant la rhinite (mais il n'y avait pas d'information si celle-ci était ou non allergique dans cette enquête). Le tableau 5 montre une relation significative de l'asthme avec l'indicateur, mais avec un profil peu clair. Pour ce 
qui est de la catégorie la plus élevée de l'indicateur, la prévalence des crises d'asthme est néanmoins, comme attendu, la plus faible, mais cette différence n'est pas statistiquement significative en comparant ce groupe à celui des communes non rurales de plus de 5000 habitants $(4,8 \%$ vs $2,9 \%$; $\mathrm{p}=0,13)$.

\section{Tableau 5.}

Relation de l'indicateur de ruralité avec l'asthme selon l'enquête PAARC

\begin{tabular}{|c|c|c|c|c|c|c|}
\hline $\begin{array}{l}\text { Indicateur } \\
\text { de ruralité } \\
\text { (contact } \\
\text { avec } \\
\text { les bovins) }\end{array}$ & $\begin{array}{l}0 \text { Com. }> \\
5000 \text { hab. }\end{array}$ & $\begin{array}{c}1 \\
\mathrm{~B} / \mathrm{H}<1\end{array}$ & $\begin{array}{c}2 \\
B / H \geq 1 \\
\text { et }<2\end{array}$ & $\begin{array}{c}3 \\
B / H \geq 2 \\
\text { et }<2,5\end{array}$ & $\begin{aligned} & 4 \\
& \mathrm{~B} / \mathrm{H} \geq 2,5 \\
& \text { et }<3\end{aligned}$ & $\begin{array}{c}5 \\
\mathrm{~B} / \mathrm{H} \geq 3\end{array}$ \\
\hline$n$ & 13213 & 2771 & 901 & 285 & 197 & 280 \\
\hline Asthme, \% & 6,2 & 5,4 & 8,7 & 6,0 & 8,1 & $5,4^{*}$ \\
\hline $\begin{array}{l}\text { Diagnostic } \\
\text { d'asthme, \% }\end{array}$ & 4,9 & 3,9 & 6,1 & 3,9 & 5,1 & $2,9^{\star}$ \\
\hline $\begin{array}{l}\text { Crise } \leq 16 \text { ans, } \\
\%\end{array}$ & 43,5 & 35,4 & 32,2 & 27,3 & 63,6 & 33,3 \\
\hline Âge $1^{\text {re }}$ crise & $\begin{array}{l}21,3 \\
(658)\end{array}$ & $\begin{array}{l}23,3 \\
(110)\end{array}$ & $\begin{array}{l}27,4 \\
(59)\end{array}$ & $\begin{array}{l}24,1 \\
(11)\end{array}$ & $\begin{array}{l}19,8 \\
(11)\end{array}$ & $\begin{array}{l}18,8 \\
(9)\end{array}$ \\
\hline Sifflements, \% & 13,9 & 13,5 & 14,4 & 10,2 & 11,1 & 14,6 \\
\hline Rhinite, \% & 10,6 & 11,4 & 11,2 & 10,9 & 10,7 & 12,1 \\
\hline
\end{tabular}

$*: p \leq 0,05$

Comparé à l'asthme féminin, l'asthme masculin est tout à la fois plus souvent précoce (début dans l'enfance) et allergique. Aussi, l'analyse a été stratifiée selon le genre (tableau 6). Chez les hommes, c'est dans les 3 catégories les plus élevées que la prévalence de crises d'asthme et de sifflements est la plus faible mais les profils observés ne sont pas très nets.

L'analyse entreprise dans l'enquête PAARC a ainsi permis de définir des indicateurs rétrospectifs de ruralité dans la petite enfance dans le contexte d'une étude épidémiologique (ce qui n'avait jamais été fait). 
Tableau 6.

Relation de l'indicateur de ruralité avec l'asthme selon les genres

\begin{tabular}{|c|c|c|c|c|c|c|}
\hline $\begin{array}{l}\text { Indicateur } \\
\text { de ruralité } \\
\text { (contact } \\
\text { avec } \\
\text { les bovins) }\end{array}$ & $\begin{array}{l}0 \text { Com. > } \\
5000 \text { hab. }\end{array}$ & $\begin{array}{c}1 \\
B / H<1\end{array}$ & $\begin{array}{c}2 \\
B / H \geq 1 \\
\text { et }<2\end{array}$ & $\begin{array}{c}3 \\
B / H \geq 2 \\
\text { et }<2,5\end{array}$ & $\begin{array}{c}4 \\
\mathrm{~B} / \mathrm{H} \geq 2,5 \\
\text { et }<3\end{array}$ & $\begin{array}{c}5 \\
B / H \geq 3\end{array}$ \\
\hline $\begin{array}{l}\text { Hommes } \\
n\end{array}$ & 5977 & 1153 & 388 & 143 & 84 & 126 \\
\hline Asthme, \% & 6,6 & 5,5 & 8,5 & 4,2 & 7,1 & 7,1 \\
\hline $\begin{array}{l}\text { Diagnostic } \\
\text { d'asthme, \% }\end{array}$ & 5,2 & 4,0 & 5,4 & 2,8 & 3,6 & 3,2 \\
\hline $\begin{array}{l}\text { Crise } \leq 16 \text { ans, } \\
\%\end{array}$ & $\begin{array}{l}51,3 \\
(310)\end{array}$ & $\begin{array}{l}45,4 \\
(44)\end{array}$ & $\begin{array}{l}36,4 \\
(22)\end{array}$ & $\begin{array}{c}50,0 \\
(4)\end{array}$ & $\begin{array}{l}100 \\
(3)\end{array}$ & $\begin{array}{c}50,0 \\
(6)\end{array}$ \\
\hline Sifflements, \% & 16,3 & 16,4 & 18,5 & 12,6 & 8,3 & 15,9 \\
\hline Rhinite, \% & 12,0 & 13,6 & 13,1 & 10,5 & 11,9 & 15,9 \\
\hline $\begin{array}{l}\text { Femmes } \\
n\end{array}$ & 7235 & 1618 & 511 & 142 & 113 & 154 \\
\hline Asthme, \% & 6,0 & 5,4 & 8,8 & 7,8 & 8,8 & $3,9^{*}$ \\
\hline $\begin{array}{l}\text { Diagnostic } \\
\text { d'asthme, \% }\end{array}$ & 4,6 & 3,8 & 6,6 & 4,9 & 6,2 & 2,9 \\
\hline $\begin{array}{l}\text { Crise } \leq 16 \text { ans, } \\
\%\end{array}$ & $\begin{array}{l}36,5 \\
(348)\end{array}$ & $\begin{array}{l}28,8 \\
(66)\end{array}$ & $\begin{array}{l}29,7 \\
(37)\end{array}$ & $\begin{array}{c}14,3 \\
(7)\end{array}$ & $\begin{array}{c}50,0 \\
(8)\end{array}$ & $\begin{array}{c}0 \\
(3)\end{array}$ \\
\hline Sifflements, \% & 11,8 & 11,5 & 11,3 & 7,7 & 13,2 & 13,6 \\
\hline Rhinite, \% & 9,4 & 9,8 & 9,8 & 11,3 & 9,7 & 9,0 \\
\hline
\end{tabular}

\section{Conclusions}

Tant du côté des géographes que des épidémiologistes, un retour sur expérience a été mené sur l'apport de la mise en commun pour formuler des nouvelles hypothèses, de nouvelles pistes méthodologiques (étude de communes? échantillon de populations et trajectoires résidentielles? validation des résultats dans d'autres milieux et d'autres temps?). 
La réflexion a aussi porté sur l'apport de la pratique interdisciplinaire sur les disciplines engagées dans l'expérience. Pour les géographes, c'est dans le dialogue avec les épidémiologistes qu'ils ont pris conscience de la normativité de l'idée que les sciences sociales se font de l'agriculture française et de ses transformations. Façonnée par les statistiques, cette idée (représentation) s'est éloignée de sa relation au vivant et au milieu physique, ce qui révèle la sous-estimation du poids du contexte historique sur la formulation des questions de recherche dans le temps. Il est apparu que malgré l'importance du travail de validation de l'indicateur de ruralité, celui-ci ne reflète peut-être pas suffisamment la complexité des expositions en cause - compte tenu des statistiques disponibles - pour rendre compte du «milieu» que l'on cherche rétrospectivement à reconstituer (le milieu de petites exploitations d'élevage en habitat groupé ou semi-groupé et où il y a de fortes probabilités de contacts précoces avec les «vaches»).

Pourtant, en dépit de son caractère sommaire, l'indicateur densité de bovins par habitant confirme, en la retrouvant, l'importance de la notion de «milieu». Les sciences sociales ont intérêt à poursuivre des recherches pour revisiter les concepts de rural et d'urbain et de la place du vivant animal dans la pensée de ces deux catégories. La recherche "ruralité et asthme» a constitué un encouragement à tester la pertinence heuristique du couple de concept «mode d'habiter » et «culture de la nature» pour explorer ce que peut vouloir dire «durabilité» quand lieu et milieu sont mis en relation avec les pratiques habitantes. Enfin, un nouvel axe de recherche environnement/santé a émergé, celui de l'aggravation potentielle et probable des problèmes de santé dus à la disparition des «contacts» avec les animaux.

Les épidémiologistes, quant à eux, attribue d'abord à la pratique interdisciplinaire un résultat majeur: la pertinence et la faisabilité de l'indicateur de ruralité dont la simplicité va de pair avec l'efficacité. Mais l'apport s'est également révélé dans l'usage de la cartographie qui a été reconnu comme outil méthodologique utilisable par des épidémiologistes pour penser la relation de leurs sujets avec les lieux et milieux. Ainsi, la carte de la densité de vaches laitières par commune de l'enquête PAARC a incontestablement compté pour enrichir, voire renouveler la représentation que ces chercheurs se faisaient de «l'espace géographique» et pour 
envisager d'introduire la méthode des matrices unité géographique/exposition pour des enquêtes utilisant des données rétrospectives (petite enfance des sujets adultes). De la longue marche pour aboutir à ces résultats, elles ont retenu qu'une relation interdisciplinaire est une relation choisie et testée durant toute la démarche: c'est avec ces géographes qu'il a été possible de travailler dans la mesure où aucun point de vue paradigmatique ne leur a jamais été opposé ou imposé. Par hypothèse, la sociologie leur semblait être la discipline partenaire, ce qui s'est avéré inexact.

En somme, les épidémiologistes, après la phase de mise en commun du vocabulaire, ont pris conscience de la richesse et de la multiplicité des approches possibles en ce qui concerne ce qui a été appelé - bien (trop) sommairement - la ruralité. Alors que des travaux biologiques et immunologiques se développent sur un plan international pour comprendre quels agents biologiques ou infectieux dans les contacts avec les animaux de ferme pourraient être responsables de l'accélération de la maturation immunologique dans la petite enfance entraînant une diminution de l'allergie, la prise en compte du contexte plus global de ces expositions et les possibilités d'une estimation rétrospective simple quand des données directes ne sont pas disponibles sont d'une grande actualité. Le travail conceptuel qui a été mené va permettre une adaptation des indicateurs à de nouvelles hypothèses. Ainsi, les données les plus récentes sur le plan immunologique suggèrent que la diversité des expositions aux agents infectieux devrait être considérée. L'approche entreprise ici permettra de définir des indicateurs rétrospectifs permettant de tester ces nouvelles hypothèses en considérant par exemple diverses espèces animales. Par ailleurs, reprendre à partir des données des sciences sociales la question de «l'habiter" peut aussi être un moyen d'appréhender les gestes qui favorisent ou repoussent le contact avec les animaux et le vivant de façon plus générale et donc l'intensité des contacts provoquant ces modifications immunologiques.

Ainsi, la pratique interdisciplinaire a ouvert un certain nombre de perspectives de recherche:

- L'application de la méthode à d'autres espèces animales rendue possible par l'approche par canton pour les petites communes qui corrige le problème des données manquantes particulièrement important pour les animaux autres que les bovins. Cette extension 
est intéressante du point de vue de l'épidémiologie car l'hypothèse que la diversité des contacts avec les animaux puisse être un élément protecteur particulièrement important y est actuellement débattue.

- L'application à d'autres enquêtes françaises de cet indicateur accompagnée d'une analyse approfondie par la mise en relation avec les questionnaires détaillés sur les contacts avec les animaux de ferme (des contrats ont été obtenus pour étudier cette question dans l'étude E3N et dans l'étude EGEA).

- Une approche "vie entière " ${ }^{10}$ est envisageable en considérant toute l'histoire résidentielle et non seulement la commune de naissance. L'apport des systèmes d'information géographique, domaine en plein développement, serait très utile dans ce contexte.

- Des développements sur un plan international sont envisageables également grâce à la mise en place des systèmes d'information géographique. Le géocodage des histoires résidentielles se développe permettant de mettre en relation les lieux de résidence avec diverses bases de données géographiques et c'est bien une telle base de données sur la question des contacts avec les bovins que nous avons construite.

Au-delà des apports de l'interdisciplinarité spécifiquement liés à cette thématique la question se pose de conclure sur la place de ce type d'expérience dans la programmation des recherches sur «Milieux de vie et santé». Le fait qu'il s'agisse d'une interdisciplinarité «choisie» sur une question "critique" avec un objectif (et donc une réponse) limité(é) et de type fondamental, à savoir une "preuve scientifique» mérite d'être réfléchi par rapport à d'autres pratiques au Nord comme au Sud. Il en est de même du passage progressif d'une entrée "populations» à une exploration de l'entrée «milieux de vie» des «habitants» mobiles dans le temps et l'espace. La pratique de l'interdisciplinarité donne l'opportunité d'évaluer nos méthodes et nos paradigmes et de trouver les déplacements nécessaires pour construire des embryons de réponse à des questions de société. 


\section{Références bibliographiques}

Hucy W., Mathieu N., Mazellier T., Raynaud H., 2005 - L'habitabilité des milieux urbains: un objet au croisement des disciplines. In Mathieu N., Guermond Y., éd.: La ville durable, du politique au scientifique, Paris, Éditions Quae, coll. Indisciplines: 237-260.

JOLlivet M., 1992 - Sciences de la nature, sciences de la société, les passeurs de frontières. Paris, Éditions CNRS.

Jolliver M., éd., 2001 - Le développement durable, de l'utopie au concept: de nouveaux chantiers pour la recherche. Paris, Amsterdam, New York, Elsevier.

JOLlivet M., LEGAY J.-M., 2005 - Canevas pour une réflexion sur une interdisciplinarité entre sciences de la nature et sciences sociales. Natures Sciences Sociétés, 13: 184-188.

Kauffmann F., Oryszczyn M.-P., Maccario J., 2002 - The protective role of country living on skin prick tests, immunoglobulin $\mathrm{E}$ and asthma in adults from the Epidemiological study on the Genetics and Environment of Asthma, bronchial hyper-responsivenes and atopy. Clin Experim Allergy, 32 : 379-86.

LEGAY J.-M., 2004 - Linterdisciplinarité vue et pratiquée par les chercheurs en Sciences de la vie". Table ronde des journées NSS 2002, Natures Sciences Sociétés, vol. 12, 1.

LEGAY J.-M., éd., 2006 - L'interdisciplinarité dans les sciences de la vie. Paris, Quae éditions, coll. Indisciplines.

MATHIEU N., 1990 - La notion de rural et les rapports ville/campagne en France, des années 1950 aux années 1980. Économie rurale, 197.

MATHIEU N., 1992 - Géographie et interdisciplinarité, rapport naturel ou rapport interdit? In Jollivet M., dir. : Sciences de la nature, sciences de la société, les passeurs de frontières, Paris, Éditions du CNRS: 129-154.

MATHIEU N., 1998 - La notion de rural et les rapports ville/campagne en France, les années 1990. Economie rurale, 247: 11-20.

MATHIEU N., 2002a - Expériences et méthodologies des observatoires. Les carnets de l'Observatoire des rapports entre le rural et l'urbain, 1-1:31.

Mathieu N., 2002 b - Expériences et méthodologies des Observatoires: un retour critique sur l'Observatoire des rapports rural/urbain. Cahiers Nantais, 58: 71-82.

Mathieu N., 2007 - L'évolution des modes d'habiter: un révélateur des mutations des sociétés urbaines et rurales. In Luginbühl Y., dir. : Nouvelles ruralités, nouvelles ruralités en Europe, Bruxelles, Bern, Berlin, Frankfurt am Main, New York, Oxford, Wien, Peter Lang: 25-45. 
Mathieu N., Blanc N., Rivault C., Cloarec A., 1997 - Le dialogue interdisciplinaire mis à l'épreuve: réflexions à partir d'une recherche sur les blattes urbaines. Natures Sciences Sociétés, 1, vol. 5: 18-30.

Mathieu N., De Lafond V., Gana A., 1998 - Towards New Responsible Rural/Urban Relationships. A Sustainable Territories-oriented Comparative Analysis. Université Paris-X, UMR Ladyss, $80 \mathrm{p}$.

Mathieu N., Guermond Y., éd., 2005 - La ville durable, du politique au scientifique. Paris, Éditions Quae, coll. Indisciplines, $286 \mathrm{p}$.

MATHIEU N., ORYSZCZYN M.-P., 2003 - «La valeur heuristique de l'interdisciplinarité choisie: exemple de pratiques entre sciences sociales et sciences de la vie». In Comité scientifique d'organisation de l'École thématique: La demarche interdisciplinaire dans le domaine de l'environnement: methodes et outils pour partager les savoirs, octobre, Aussois.

Mathieu N., Robert M., 1998 - Pourquoi un observatoire des rapports urbain/rural? Les carnets de l'Observatoire des rapports entre rural et urbain, $1^{\text {er }}$ décembre, $6 \mathrm{p}$.

Oryszczyn M.-P., Mathieu N., Le Moual N., Kauffmann F., 2003 «Rurality of birth place and asthma. Preliminary results in 18,000 adults from the French PAARC survey based on retrospective indicators of rurality for 4,000 birth places ». In: Abstract $n^{\circ} 455$, XXII Congress of European Academy of Allergology and Clinical Immunology, 7-11 juin.

ORYSZCZYN M.-P. et al., 2004 - «Histoire résidentielle et environnement de la ferme». In: Séminaire EGEA, Sausset-les-Pins.

Oryszczyn M.-P., Mathieu N., Hucy W., Maccario J., Kauffmann F, 2005 - « Geographical assessment of contact with livestock. Influence of area sampling. Preliminary results ". In : ATS Congress (American Thoracic Society), San Diego, May 2005. Proc Am Thorac Soc 2005; 2 : A851

ORYSZCZYN M.-P. (coord. sc.), MATHIEU N., HUCY W., KAUfFMANN F, 2007 - Ruralité et asthme - Approche interdisciplinaire. Recherche d'indicateurs rétrospectifs de ruralité ( 4000 communes françaises) et application à l'étude épidémiologique du rôle protecteur des contacts avec les animaux de ferme dans l'asthme dans l'enquête PAARC (18000 sujets). Rapport final, Action thématique concertée, Environnement et Santé, année 2003, Villejuif, Inserm U 780, 13 p. + annexes.

RAYNAUT C., 2001 - L'anthropologie de la santé, carrefour de questionnements: l'humain et le naturel, l'individuel et le social. Ethnologies comparees, $\mathrm{n}^{\circ}$ 3. http://alor.univ-montp3.fr/cerce/revue.htm

Robic M.-C., MATHiEU N., 2001 - «Géographie et durabilité: redéployer une expérience et mobiliser de nouveaux savoir-faire ». In Jollivet $\mathrm{M}$., éd. : Le développement durable, de l'utopie au concept: de nouveaux chantiers pour la recherche, Paris, Amsterdam, New York, Elsevier: 167-190. 


\section{Partie 2}

Représentations des risques

liés à la pollution 



\section{Quand la santé publique redéfinit les enjeux de la pollution atmosphérique en France}

\section{Franck BOUTARIC}

Pierre LASCOUMES

La loi sur l'air et l'utilisation rationnelle de l'énergie (Laure) consacre la redéfinition et la mise en cuvre d'une nouvelle politique publique dans le domaine de la lutte contre la pollution atmosphérique (BOUTARIC et al., 2002). Lobjet de cet article est de montrer comment la mobilisation des spécialistes de la santé publique a reconfiguré radicalement l'enjeu de la pollution atmosphérique par la construction et la prise en compte de son impact sanitaire. Les dimensions de l'enjeu, les instruments de sa mesure, le champ des acteurs concernés et le type de décision publique ont ainsi connu des déplacements majeurs. Notre méthode a consisté à retracer, à comprendre et à analyser les effets des recherches et des actions entreprises par les professionnels de santé et plus particulièrement les épidémiologistes. Notre démarche se présente donc en trois temps. Un bref rappel de l'émergence (ou de la ré-émergence) de l'enjeu de la pollution atmosphérique, une présentation synthétique des actions de la nouvelle épidémiologie, et un exposé de ses effets politiques. 


\section{La reconfiguration de la pollution atmosphérique comme enjeu public en France}

Plusieurs travaux ont expliqué la faible visibilité de la pollution atmosphérique comme enjeu public et comme problème politique jusqu'au début des années 1990. La gestion éclatée de la pollution atmosphérique et la captation de cette question par des structures d'expertises proches des milieux industriels ont ainsi été des causes bien explicitées par des recherches antérieures (Vlassopoulou, 1999). Parmi la diversité des facteurs susceptibles d'expliquer le faible intérêt pour cette question au cours des années 1970 et 1980, figure l'enquête «Pollution atmosphérique et affections respiratoires chroniques " (PAARC). Cette enquête a marqué la communauté scientifique française (FESTY et QUENEL, 2003), car elle montre, somme toute, une relation modeste entre la pollution atmosphérique et la prévalence de troubles respiratoires. Une conclusion se dégage: grâce à la politique de réduction des émissions (industrielles et des appareils de chauffage) engagée depuis la loi de 1961, les effets sanitaires de la pollution atmosphérique ne constituent pas un enjeu de santé publique.

En France, il a fallu attendre la revue de littérature scientifique internationale de l'année 1992 réalisée dans le cadre du programme Erpurs (Évaluation des risques de la pollution urbaine pour la santé) et la période du développement des études épidémiologiques écologiques temporelles (1990-2000) pour qu'apparaisse à nouveau un regain d'intérêt pour la pollution atmosphérique et ses effets sur la santé.

Les études écologiques temporelles emploient des méthodes d'analyse statistiques qui soulignent de manière fine les relations entre indicateurs de pollution et indicateurs sanitaires et permettent de contrôler des facteurs de confusion. Ainsi, la pollution atmosphérique a été constituée (en partie reconstituée après l'épisode de la fin des années 1950) comme un enjeu de santé publique. L'histoire de cette ré-émergence présente un processus similaire à celui démontré par Didier Fassin à propos du saturnisme. On constate la production de connaissances nouvelles, une 
modification du regard et une nouvelle représentation de la pollution atmosphérique, toutes étroitement associées à la mise en œuvre d'une nouvelle épidémiologie. Cela montre bien que les problèmes sanitaires ne sont pas seulement des réalités biologiques que les spécialistes viennent objectiver, ils sont aussi des faits épidémiologiques construits par des savoirs et des acteurs (FASSIN, 2005).

\section{La nouvelle épidémiologie en action}

Dans le champ de l'épidémiologie émerge une nouvelle approche dans la période 1990-2000. Elle repose sur une méthode qui est appliquée au domaine de la pollution atmosphérique urbaine. Il y a concomitance entre la diffusion de cette méthode et la reconfiguration du problème public de la pollution atmosphérique par sa requalification en enjeu de santé publique. Sans l'utilisation de cet outil, les corrélations entre les indicateurs de pollution et ceux de santé n'auraient pu être rendues tangibles et les interrogations sur l'existence de liens causaux entre eux ne se seraient pas posées avec une telle acuité.

Dans un premier temps, nous présentons une brève synthèse des études épidémiologiques, dans un deuxième temps, nous exposerons quelques-unes des controverses suscitées par la publication de ces travaux.

Le tableau 1 indique que les différentes évaluations sont produites dans un cadre régional. Des quatre études qui à ce jour ont été réalisées, celle de l'année 1994 est historiquement la plus importante. Elle a exercé un rôle majeur dans la reconfiguration en enjeu de santé publique et a fortement marqué les milieux de la santé publique et l'ensemble des agents intéressés par la gestion de la pollution atmosphérique en France. C'est également la première fois qu'en France, on emploie une méthode d'analyse qualifiée « d'études écologiques et temporelles». Cette méthode d'analyse statistique a été élaborée dans le cadre d'un projet européen associant les chercheurs de quinze villes européennes. Le raisonnement utilisé 
Tableau 1.

Évaluation des risques de la pollution urbaine sur la santé, région île-de-France

\begin{tabular}{|c|c|c|c|c|}
\hline & Études & $\begin{array}{l}\text { Indicateurs } \\
\text { de pollution }\end{array}$ & $\begin{array}{l}\text { Indicateurs } \\
\text { sanitaires }\end{array}$ & $\begin{array}{l}\text { Méthode } \\
\text { d'analyse }\end{array}$ \\
\hline $\begin{array}{l}\text { Erpurs } \\
1994\end{array}$ & $\begin{array}{l}\text { Evaluation de l'impact } \\
\text { de la pollution urbaine } \\
\text { sur la santé } \\
\text { en île-de-France } \\
(1987-1992)\end{array}$ & $\begin{array}{l}\mathrm{FN}, \mathrm{PS}(13) \\
\mathrm{SO}_{2}, \mathrm{O}_{3}, \mathrm{NO}_{2}\end{array}$ & $\begin{array}{l}\text { Données de mortalité totale et liées } \\
\text { aux maladies de l'appareil respiratoire } \\
\text { et de l'appareil circulatoire. } \\
\text { Données de morbidité (hospitalisation, } \\
\text { urgences pédiatriques, } \\
\text { visites médicales à domicile, } \\
\text { absentéisme professionnel). }\end{array}$ & $\begin{array}{l}\text { Études écologiques et temporelles. } \\
\text { La méthode d'analyse statistique } \\
\text { retenue est celle qui a été élaborée } \\
\text { dans le cadre du projet APHEA } \\
\text { par des chercheurs de } 15 \text { villes } \\
\text { européennes. }\end{array}$ \\
\hline $\begin{array}{l}\text { Erpurs } \\
1998\end{array}$ & $\begin{array}{l}\text { Analyse des liens } \\
\text { à court terme entre } \\
\text { pollution atmosphérique } \\
\text { et santé (1991-1995) }\end{array}$ & Idem & Idem & $\begin{array}{l}\text { Le protocole d'analyse statistique } \\
\text { a été amélioré et mis au point } \\
\text { dans le cadre du projet européen } \\
\text { APHEA. }\end{array}$ \\
\hline $\begin{array}{l}\text { Erpurs } \\
2003\end{array}$ & $\begin{array}{l}\text { Analyse des liens } \\
\text { à court terme entre } \\
\text { niveaux de pollution } \\
\text { atmosphérique et } \\
\text { visites médicales à domicile }\end{array}$ & $\begin{array}{l}\mathrm{FN}, \mathrm{NO}_{2}, \mathrm{O}_{3} \\
\mathrm{SO}_{2}, \mathrm{PM} 13 \\
\mathrm{PM}_{10}\end{array}$ & $\begin{array}{l}\text { Indicateurs de mortalité: idem } \\
\text { Indicateurs de morbidité: } \\
\text { causes d'hospitalisation. }\end{array}$ & $\begin{array}{l}\text { La méthode d'analyse statistique } \\
\text { bénéficie des améliorations apportées } \\
\text { au cours des études nationales } \\
\text { (PSAS 9) et européennes (APHEIS). }\end{array}$ \\
\hline $\begin{array}{l}\text { Erpurs } \\
2005\end{array}$ & $\begin{array}{l}\text { Analyse des liens } \\
\text { à court terme entre } \\
\text { niveaux de pollution } \\
\text { atmosphérique et } \\
\text { visites médicales à domicile }\end{array}$ & $\begin{array}{l}\mathrm{NO}_{2}, \mathrm{PM} 10 \\
\mathrm{PM} 2,5\end{array}$ & $\begin{array}{l}\text { Nombre journalier d'appels } \\
\text { pour l'asthme, pour affection } \\
\text { des VRI et des VRS. }\end{array}$ & \\
\hline
\end{tabular}

FN: fumées noires; PS : particules en suspension; PM: particules fines. 
Tableau 2.

APHEA - Air pollution and health : a European approach

\begin{tabular}{|c|c|c|c|}
\hline & Date de lancement & Objectifs retenus ${ }^{1}$ & Publications \\
\hline APHEA 1 & $\begin{array}{l}\text { - } 1991 \\
\text { - } 1993, \\
\text { Intégration de l'équipe } \\
\text { d’Erpurs }\end{array}$ & $\begin{array}{l}\text { - Élaborer une méthodologie standardisée } \\
\text { et commune aux } 15 \text { villes européennes } \\
\text { (étude écologique et temporelle). } \\
\text { - Analyse des causes de décès } \\
\text { et d'admission hospitalière en relation } \\
\text { avec les concentrations de polluants } \\
\text { ( } \mathrm{FN}, \mathrm{SO}_{2}, \mathrm{NO}_{2}, \mathrm{O}_{3} \text { ). }\end{array}$ & $\begin{array}{l}\text { - Plus d'une vingtaine d'articles publiés. } \\
\text { - Méthodologie, protocole: } \\
1994 \text { : travaux de J. Schwartz } \\
1996 \text { : Short-term effects of air pollution on health } \\
\text { a European approach using epidemiological } \\
\text { time series data: the Aphea protocol. } \\
\text { - Présentation des résultats sous forme de risque } \\
\text { attribuable. } \\
\text { 1995: impact de la pollution atmosphérique } \\
\text { urbaine de type acido-particulaire sur la mortalité } \\
\text { quotidienne à Lyon et dans l'agglomération } \\
\text { parisienne. }\end{array}$ \\
\hline APHEA 2 & $\begin{array}{l}\text { - } 1998 \\
\text { - Collaboration } \\
\text { de l'équipe de PSAS } 9\end{array}$ & $\begin{array}{l}\text { - Mettre en evidence les populations sensibles, } \\
\text { identifier les facteurs qui expliqueraient } \\
\text { les différences entre les villes. } \\
\text { - Mettre au point des fonctions } \\
\text { expositions/risques pour une exposition } \\
\text { à court terme. } \\
\text { - Contribuer au plan international à la révision } \\
\text { des valeurs limites d'exposition. }\end{array}$ & $\begin{array}{l}\text { - Articles sur les populations sensibles, } \\
\text { les effets des particules sur les maladies } \\
\text { cardiovasculaires. } \\
\text { - Prise en compte d'un plus grand nombre } \\
\text { de polluants. }\end{array}$ \\
\hline
\end{tabular}

(1) Les tableaux n'ont pas pour but d'énumérer l'ensemble des objectifs de programmes qui s'échelonnent sur plusieurs années. Nous avons donc opéré une sélection qui s'attache aux aspects novateurs. 
est différent de celui des études épidémiologiques dites classiques. Le principe de ces études (...) est de comparer les risques quotidiens de décès ou d'hospitalisations pour des jours plus ou moins pollués. L'unité d'observation n'est donc pas l'individu, mais la journée (GÉRIN et al., 2003: 300).

Ce recours à une nouvelle méthodologie standardisée, et commune à plusieurs villes européennes, s'appuie sur les travaux élaborés en Amérique du Nord. C'est ce qu'illustre la participation du biostatisticien Joël Schwartz à ce projet; ces études menées aux États-Unis pour l'essentiel sont devenues des références incontournables pour la communauté des épidémiologistes environnementaux (SCHWARTZ, 1994). Initiée en Europe, au début des années 1990, cette approche a donné lieu à une vingtaine de publications. En France, l'article de QUENEL et al. (1995) a eu un retentissement important. La présentation des résultats sous la forme de risque attribuable et leur diffusion par la presse suscitent de fortes controverses autant sur les méthodes d'analyses que sur le recours aux médias pour exposer des conclusions scientifiques. L'année 1995 marque incontestablement un tournant dans l'intérêt et la prise en charge par les "autorités publiques», de la question de la pollution atmosphérique urbaine (FESTY, QUENEL, 2003). Une partie des travaux d'APHEA 2 concerne la mise au point des fonctions expositions-risques pour une exposition à court terme. Cette étude est aussi réalisée avec le programme de surveillance Air/Santé, coordonné nationalement par l'Institut de veille sanitaire, elle ouvre la possibilité d'évaluer l'impact sanitaire de polluants atmosphériques (tabl. 2).

Avec le Programme de surveillance air et santé (PSAS) 9, nous passons de la mise en place d'un dispositif de surveillance épidémiologique à l'évaluation d'impact sanitaire. Dans un premier temps, l'évaluation d'impact sanitaire concerne le court terme. On observe que les indicateurs sanitaires se diversifient et que la mesure des PM 10 est introduite parmi les indicateurs de pollution (tabl. 3).

L'approfondissement de la mesure des polluants et l'évaluation des risques se poursuivent. Ils s'opèrent en particulier dans le cadre d'une étude au titre significatif des évolutions opérées dans le domaine de la pollution atmosphérique: "Surveillance épidémiologique et évaluation des impacts sanitaires» (tabl. 4). 
Tableau 3.

Programme de surveillance air et santé

\begin{tabular}{|c|c|c|c|c|}
\hline & Dates & Objectifs retenus $^{1}$ & $\begin{array}{l}\text { Indicateurs } \\
\text { de pollution }\end{array}$ & $\begin{array}{l}\text { Indicateurs } \\
\text { sanitaires }\end{array}$ \\
\hline $\begin{array}{l}\text { PSAS } \\
\text { dans } 9 \text { villes }\end{array}$ & $\begin{array}{l}\text { - Lancement en } 1997 \\
\text { - Publication du rapport } \\
\text { d'étude en mars } 1999\end{array}$ & $\begin{array}{l}\text { - Étude de faisabilité de la mise en place } \\
\text { d'un dispositif de surveillance épidémiologique } \\
\text { dans } 9 \text { villes. } \\
\text { - Analyser les effets à court terme } \\
\text { de la pollution atmosphérique dans } 9 \text { villes. }\end{array}$ & $\mathrm{FN}, \mathrm{SO}_{2}, \mathrm{NO}_{2}, \mathrm{O}_{3}$ & $\begin{array}{l}\text { Mortalité totale } \\
\text { non accidentelle, } \\
\text { mortalité respiratoire } \\
\text { et cardiovasculaire. }\end{array}$ \\
\hline $\begin{array}{l}\text { PSAS } \\
\text { dans } 9 \text { villes }\end{array}$ & $\begin{array}{l}\text { Publication du rapport } \\
\text { d'étude en juin } 2002\end{array}$ & $\begin{array}{l}\text { - Valider et préciser les relations } \\
\text { expositions/risques à court terme } \\
\text { entre les indicateurs de pollution } \\
\text { et les indicateurs sanitaires. } \\
\text { - Quantification de l'impact sanitaire } \\
\text { à court terme de la pollution atmosphérique } \\
\text { sur les } 9 \text { villes. }\end{array}$ & $\begin{array}{l}\mathrm{FN}, \mathrm{SO}_{2}, \mathrm{NO}_{2}, \mathrm{O}_{3}, \\
\mathrm{PM} 10, \mathrm{PM} 13\end{array}$ & $\begin{array}{l}\text { - Mortalité totale } \\
\text { (hors accidents } \\
\text { et morts violentes), } \\
\text { mortalité respiratoire } \\
\text { et cardiovasculaire. } \\
\text { - Morbidité: } \\
\text { trois indicateurs } \\
\text { d'admissions hospitalières. }\end{array}$ \\
\hline PSAS-9 & $\begin{array}{l}\text { Publication } \\
2004-2005\end{array}$ & $\begin{array}{l}\text { Analyser les relations température, } \\
\text { pollution atmosphérique et mortalité } \\
\text { (canicule d'août 2002). }\end{array}$ & $\mathrm{O}_{3}$ & Mortalité totale \\
\hline
\end{tabular}

(1) Les tableaux n'ont pas pour but d'énumérer l'ensemble des objectifs de programmes qui s'échelonnent sur plusieurs années. Nous avons donc opéré une sélection qui s'attache aux aspects novateurs. 
Tableau 4.

APHEIS - Air pollution and health: a European information system, surveillance épidémiologique et évaluation d'impacts sanitaires

\begin{tabular}{|c|c|c|c|}
\hline & Objectifs retenus $^{1}$ & $\begin{array}{l}\text { Indicateurs } \\
\text { de pollution }\end{array}$ & $\begin{array}{l}\text { Indicateurs } \\
\text { sanitaires }\end{array}$ \\
\hline $\begin{array}{l}\text { APHEIS I } \\
\text { Rapport d'étude, } \\
\text { mars } 2001\end{array}$ & $\begin{array}{l}\text { - Définition des indicateurs appropriés } \\
\text { pour la surveillance et la réalisation de l'EIS/PA en Europe. } \\
\text { - Identification des identités aptes à mener le projet. }\end{array}$ & & \\
\hline $\begin{array}{l}\text { APHEIS } 2 \\
\text { Rapport d'étude, } \\
\text { octobre } 2002\end{array}$ & $\begin{array}{l}\text { - Evaluation des impacts sanitaires des PM } 10 \\
\text { et des FN à court et long terme. }\end{array}$ & FN, PM 10 & $\begin{array}{l}\text { - Mortalité totale. } \\
\text { - Admissions hospitalières } \\
\text { (villes avec admissions } \\
\text { aux urgences et villes } \\
\text { avec admissions générales). }\end{array}$ \\
\hline $\begin{array}{l}\text { APHEIS } 3 \\
\text { Rapport d'étude, } \\
\text { juillet } 2004\end{array}$ & $\begin{array}{l}\text { - Exploitation de la fonction exposition/risque } \\
\text { utilisée dans l'évaluation d'impact sanitaire effectuée } \\
\text { dans trois pays européens pour l'exposition } \\
\text { à long terme à la pollution atmosphérique. } \\
\text { - Gains sanitaires attendus des différents scenarii } \\
\text { de réduction des PM } 2,5 \\
\text { (utilisation fonction expo/risque de Pope 2002). } \\
\text { - Dispositif d'information pour les décideurs locaux } \\
\text { et européens. }\end{array}$ & FN, PM 10, PM 2,5 & $\begin{array}{l}\text { - Mortalité : } 3 \text { indicateurs. } \\
\text { Mortalité totale hors accidents } \\
\text { et morts violentes; } \\
\text { mortalité pour causes respiratoires } \\
\text { et pour causes cardiovasculaires. } \\
\text { - Admissions hospitalières: } \\
\text { pour pathologies cardiaques } \\
\text { et respiratoires. }\end{array}$ \\
\hline
\end{tabular}

(1) Les tableaux n'ont pas pour but d'énumérer l'ensemble des objectifs de programmes qui s'échelonnent sur plusieurs années. Nous avons donc opéré une sélection qui s'attache aux aspects novateurs. 
D'importants travaux sont menés pour définir les indicateurs appropriés à la surveillance de la qualité de l'air dans 26 villes européennes et le rapport de 2003 effectue les calculs de gains sanitaires attendus des différents scénarios de réduction d'un polluant (PM 2,5). Cette étude reprend, prolonge et approfondit celle menée dans le cadre de PSAS 9 avec une innovation qui propose un dispositif de diffusion des résultats des travaux scientifiques auprès des décideurs locaux et européens.

Cette dynamique se poursuit à deux niveaux. Tout d'abord par la réalisation d'études qui permettent d'évaluer les impacts sanitaires à long terme. Ensuite, cette progression de la connaissance est renforcée par la mise à disposition des données relatives aux impacts de la pollution et par la réalisation de guides méthodologiques par l'Institut de veille sanitaire (tabl. 5).

Si en 1994, de nombreuses interrogations subsistaient quant aux effets de la pollution atmosphérique sur la santé et que l'on débattait encore sur le lien de causalité entre pollution atmosphérique urbaine et indicateurs de mortalité ou de morbidité, ces incertitudes apparaissent dépassées dès 1999 où le premier guide méthodologique de l'InVS estime les gains sanitaires potentiels selon différents scénarios. Durant la période 1999 à 2003, la démarche méthodologique se précise en intégrant d'autres indicateurs et en s'élargissant aux effets à long terme. La lecture rapide des tableaux ci-dessus permet de dresser plusieurs constats.

Des échelons territoriaux variés se sont impliqués: des villes, des régions, des organismes nationaux et européens sont engagés dans ces études. Le rythme des études est assez soutenu et implique un nombre croissant d'acteurs et d'institutions. De 1994 à 2003, on ne peut qu'être frappé par l'approfondissement d'une démarche qui initialement se basait sur des indicateurs de pollution et d'activités sanitaires parfois difficilement recueillis.

L'évaluation des impacts sanitaires est devenue la question centrale. Les Evaluations d'impacts sanitaires (EIS) estiment le nombre de cas attribuables (mortalité, morbidité) aux effets de la pollution atmosphérique. Ces cas attribuables sont estimés par rapport à un niveau de référence et sont donc potentiellement évitables si les niveaux de pollution sont ramenés à un niveau de référence 
Tableau 5.

Évaluation de l'impact sanitaire de la pollution atmosphérique urbaine

\begin{tabular}{|c|c|c|c|c|}
\hline Guides & Institution & Objectifs retenus ${ }^{(1)}$ & $\begin{array}{l}\text { Indicateurs } \\
\text { de pollution }\end{array}$ & $\begin{array}{l}\text { Indicateurs } \\
\text { sanitaires }\end{array}$ \\
\hline $\begin{array}{l}\text { Guide méthodologique, } \\
\text { juillet } 1999\end{array}$ & $\begin{array}{l}\text { Institut } \\
\text { de veille sanitaire }\end{array}$ & $\begin{array}{l}\text { - Quantifier localement l'impact sanitaire } \\
\text { de la pollution atmosphérique. } \\
\text { - Estimer les gains sanitaires potentiels } \\
\text { selon différents scénarios de réduction } \\
\text { de la pollution atmosphérique. }\end{array}$ & $\mathrm{SO}_{2}, \mathrm{NO}_{2}, \mathrm{O}_{3}, \mathrm{FN}$ & $\begin{array}{l}\text { Mortalité anticipée } \\
\text { et admissions } \\
\text { hospitalières. }\end{array}$ \\
\hline $\begin{array}{l}\text { Guide méthodologique, } \\
\text { version juin } 2003\end{array}$ & Idem & $\begin{array}{l}\text { Impact à court terme et à long terme } \\
\text { (PM 10) de la pollution atmosphérique. }\end{array}$ & $\begin{array}{l}\mathrm{SO}_{2}, \mathrm{NO}_{2}, \mathrm{O}_{3}, \mathrm{FN} \\
\mathrm{PM} 10\end{array}$ & $\begin{array}{l}\text { - Mortalité: } \\
\text { mortalité totale } \\
\text { hors morts violentes } \\
\text { et accidentelles, } \\
\text { mortalité } \\
\text { cardiovasculaire } \\
\text { et respiratoire. } \\
\text { - Admissions } \\
\text { hospitalières. }\end{array}$ \\
\hline
\end{tabular}

(1) Les tableaux n'ont pas pour but d'énumérer l'ensemble des objectifs de programmes qui s'échelonnent sur plusieurs années. Nous avons donc opéré une sélection qui s'attache aux aspects novateurs. 
inférieur. Cela suppose l'existence d'un lien de causalité: cette question est précisément celle qui fait l'objet de débats et de polémiques. Si la consultation des travaux mentionnés dans les tableaux fait apparaître de substantielles avancées dans la mise en évidence du lien de causalité, les discussions qui se développent lors des publications de plusieurs études démontrent que le passage du risque relatif au nombre de cas attribuables est loin de faire l'unanimité.

Dans l'histoire des controverses provoquées par les publications des études écologiques et temporelles, quatre d'entre elles sont significatives soit par l'écho qu'elles rencontrent, soit par la qualité des revues qui ouvrent leurs colonnes aux débats.

Le premier exemple date de l'année 1994 lors de la parution du rapport d'Erpurs. En prévision d'une conférence de presse destinée à rendre compte des premiers résultats, des chercheurs sont sollicités pour modifier la présentation de leurs résultats. À cette époque, les pressions émanent surtout de secteurs administratifs ou politiques inquiets des répercussions de la constitution d'un nouvel enjeu de santé publique. On peut également penser que l'élaboration de nouvelles méthodes suscite d'autant plus de circonspections que l'opinion selon laquelle la lutte contre la pollution atmosphérique a produit des résultats significatifs est alors largement répandue.

Une autre controverse publique éclate en 1996 à l'occasion de la reprise par les médias, notamment par le journal Le Monde, d'une étude menée dans le cadre du programme APHEA. Un éditorial de la revue Natures, Sciences, Sociétés $(1996,4,2)$ met sévèrement en cause la manière dont les scientifiques s'adressent aux médias et reproche aux auteurs de l'étude de laisser croire à de nouveaux résultats mettant en évidence une relation directe de cause à effet entre pollution de l'air et mortalité au sein de l'ensemble de la population. La réplique est donnée par W. DAB et al. (NSS, $1996,4,4)$. Ils affirment que leurs résultats montrent que la relation entre la pollution atmosphérique et la mortalité peut être directe et causale et que c'est la première fois que (...) des résultats épidémiologiques sont présentés sous forme de risque attribuable, ce qui permet d'évaluer l'impact de santé publique des pollutions atmosphériques. 
La question du lien de causalité est à nouveau abordée lors des travaux de PSAS 9 où le passage du risque relatif au nombre de cas attribuables est l'objet d'échanges entre les chercheurs (VAzEILles, 2003). Les discussions illustrent que les définitions relatives à l'analyse de risque ou à l'évaluation d'impact sanitaire ne sont pas immédiatement les mêmes pour tous. Les raisons de la pluralité possible des définitions trouvent leur fondement dans la diversité des formations scientifiques des épidémiologistes et de leurs méthodes de travail (VAzEILles, 2003). À cela s'ajoute la redéfinition permanente que connaissent les outils employés par les épidémiologistes. Au-delà des points de vue susceptibles d'opposer les épidémiologistes «classiques » à ceux qui utilisent les études écologiques et temporelles, les constantes évolutions des méthodes d'analyse appellent des confrontations indispensables à l'appropriation des connaissances et au dépassement des différentes perceptions. Le suivi des travaux de PSAS9 révèle que les mini-controverses ne portent pas seulement sur les aspects scientifiques et méthodologiques des travaux, elles expriment aussi des positionnements différents par rapport à l'écho que les études sont susceptibles de rencontrer auprès des décideurs. Présenter un risque relatif de l'ordre de 1,003 ou des taux de décès anticipés selon différents niveaux d'indicateur de pollution sont autant de formulations qui peuvent influencer la réception et l'importance que l'on accorde à l'enjeu de la pollution atmosphérique.

Cette controverse relative au nombre de cas attribuables et plus précisément au nombre de décès anticipés causés par la pollution atmosphérique est à nouveau publiquement discutée dans les colonnes d'une nouvelle revue scientifique: Environnement, Risques et Santé (ERS, 2003, 2, 1). La fiabilité des calculs des risques relatifs comme la légitimité du calcul des risques attribuables est discutée de manière argumentée et précise. Mais, les interpellations que Jacques ESTĖVE (2003) adresse aux auteurs d'une nouvelle étude de PSAS 9 sont moins virulentes que celles de Natures, Sciences, Sociétés. Le questionnement est sans concession, mais le ton est plus serein. La réponse détaillée des auteurs de PSAS 9 se conclut par la volonté de poursuivre échanges et approfondissement méthodologique (ERS, 2003, 2, 2). Cette interpellation qui demande s'il est «impossible de s'arrêter de comp- 
ter les morts et de réfléchir calmement sur la réalité et sur la quantification du risque de la pollution atmosphérique», nous semble pouvoir faire l'objet d'un relatif consensus. Les chercheurs de PSAS 9 n'entendent pas "s'arrêter de compter les morts". Cela reviendrait, selon nous, à atténuer la portée de dix ans de travaux d'évaluation d'impact sanitaire. Il est donc légitime de penser qu'ils ne désirent pas renier les démarches accomplies quant aux effets mortels de la pollution atmosphérique. Si aujourd'hui, la pollution atmosphérique est un enjeu, la focalisation sur le nombre de morts y a contribué. Néanmoins, les épidémiologistes, contraints de travailler avec les indicateurs dont ils disposent (la mortalité est de ceux-là), veulent diversifier leurs indicateurs. C'est ce à quoi ils s'attellent, notamment à la prise en compte de la consommation médicamenteuse, même si la diversification des données nécessaires à ces travaux n'est pas de leur seul ressort.

Ces quatre controverses sur la question de la portée des études épidémiologiques écologiques temporelles et des évaluations d'impacts sanitaires appellent plusieurs remarques. Désaccords et polémiques ont perduré pendant près de dix ans (1994-2003), mais les discussions sont désormais moins vives et les termes du débat sont circonscrits. Plusieurs facteurs peuvent expliquer ce changement.

La période du début des années 1990 est marquée d'une part par les oppositions manifestées à l'encontre des études épidémiologiques écologiques temporelles et d'autre part, par la virulence des débats préparatoires à l'adoption de la loi sur l'air et de ses décrets d'application. Les industriels émetteurs de polluants (sources fixes ou mobiles), les professions de santé publique, les associations de protection de l'environnement, bref un nombre important d'acteurs, expriment des représentations et des orientations substantiellement différentes quant à la lutte contre la pollution atmosphérique. Les questions proprement scientifiques ne sont pas absentes des discussions, mais celle du recours aux médias biaise et obscurcit parfois le sens et la nature des oppositions dans un contexte fortement politisé. Cela explique en partie la vivacité de la polémique dans la revue Natures, Sciences, Sociétés. 
Par la suite, notamment avec le vote de la loi sur l'air, le jeu des contraintes évolue. En outre, l'observateur attentif peut lier l'apaisement des tensions au fait que la légitimité change de camp, que de nouvelles représentations s'affirment. Il est symptomatique de constater que, se référant à un éditorial de la Revue épidémiologique et de santé publique, Jacques Estève (2003) l'apprécie comme "une tentative bien modeste de remise en cause du dogme», signifiant ainsi l'autorité conquise par les praticiens de la recherche épidémiologique. Nous sommes éloignés de la condescendance ou des doutes affichés à l'encontre des études écologiques temporelles lors du milieu des années 1990. Moins de dix ans après, elles acquièrent une telle légitimité qu'elles peuvent produire des dogmes.

Les raisons de cette évolution sont à rechercher du côté du perfectionnement des méthodes et de la lente construction d'un consensus qui s'opère d'une part, dans les congrès des sociétés savantes (par exemple, les épidémiologistes environnementaux) et d'autre part, dans les échanges interdisciplinaires croissants depuis la première étude d'Erpurs (ERS, 2003, 2, 2: 85). Participent aussi de la mise en place d'une configuration plus favorable aux études écologiques et temporelles, la création et le renforcement d'institutions dans le domaine de la sécurité sanitaire et environnementale tel l'InVS (DAB et al., 2006). Enfin, malgré la lente appropriation de la révolution de l'expertise en France, le renouvellement de l'approche du risque contribue à rediscuter de la question de la causalité dans des termes autres que ceux où prévalent la preuve biologique ou une interprétation restrictive des critères de HILl (1965).

Pour autant, les tensions qui accompagnent la publication des études épidémiologiques sont encore persistantes. Cela exprime la contradiction entre une science dont les résultats seraient négligés et celle dont les résultats seraient amplifiés. Cette polarité s'accentue d'autant plus que pèse une obligation d'information auprès du public et que les outils d'aide à la décision font partie de l'idéologie et de la boîte à outils de la nouvelle gouvernance. Si les controverses scientifiques ne relèvent pas exclusivement de logiques propres au champ scientifique, nous observons qu'elles produisent aussi des effets sur d'autres champs notamment celui des politiques et des décideurs. 


\section{Les effets politiques de la redéfinition sanitaire}

Dès 1994, date de la publication des premiers travaux d'Erpurs, les épidémiologistes font l'expérience du caractère imbriqué des travaux scientifiques et des enjeux publics. Leurs recherches démontrent que des risques relatifs faibles peuvent induire un risque collectif important. Mais la faiblesse initiale de la toxicologie et de l'épidémiologie environnementale comme la gestion traditionnelle d'enjeux sanitaires, approches marquées par des mortalités provoquées par des pathologies infectieuses, en partie héritées du passé, ne favorisent pas une nouvelle approche de la santé publique. Les conclusions de leurs travaux se heurtent donc au scepticisme et à l'hostilité d'intérêts bien compris. La défense de leurs méthodes comme de leurs conclusions les engage, à plusieurs reprises, sur le terrain de l'action publique et de la politique entendue dans son sens large.

En 1994, lors des débats et des concertations préparatoires à l'adoption de la loi sur l'air et l'utilisation rationnelle de l'énergie, les professionnels de la santé tentent de faire reconnaître les critères de santé publique dans la prise de décision. Corinne Lepage, alors ministre de l'Environnement, reconnaît que sans le secours d'une partie du corps médical, et en particulier de la société française de santé publique, elle n'aurait eu aucune aide extérieure durant l'élaboration de la loi (Lepage, 1998: 71). Cette volonté de faire valoir les aspects décisionnels de leurs travaux est également attestée dans le programme APHEIS qui prévoit la mise en place d'un système d'information auprès des décideurs locaux et européens. Plus récemment à propos de l'adoption de nouvelles directives, les chercheurs d'APHEIS ont fait publiquement connaître leur opposition à des projets dont l'adoption constituerait un grave recul dans l'action engagée depuis des décennies par nos pays pour réduire l'impact (...) de la pollution atmosphérique sur la santé publique" (lettre du 25-02-06, www.apheis).

Pour une bonne part, ces interventions dans le débat public s'appuient sur une nouvelle gestion du risque qui peu à peu se développe en France et en Europe. Si dès 1983, l'Académie des sciences américaine propose une démarche d'analyse des risques, 
et fournit un outil standardisé d'aide à la décision à ceux qui doivent gérer les risques environnementaux, l'appropriation de cette approche est plus lente en France. Il faut attendre 1993 pour que l'Union européenne reprenne les quatre étapes de l'évaluation des risques qui servent explicitement de base à la mise en œuvre $\mathrm{du}$ règlement $\mathrm{REACH} \mathrm{H}^{1}$. Cette démarche inspire également le programme APHEIS. La prise en compte des nouveaux enjeux environnementaux et de santé publique modifie substantiellement la communauté de politique publique en charge de la gestion de la pollution atmosphérique. Son élargissement rompt avec le monopole de gestion du problème par les milieux industriels et les Directions régionales de l'industrie et de la recherche (Drire) qui prévalait depuis les années 1960. La discussion des orientations nationales à mettre en ouvre pour lutter contre la pollution atmosphérique s'opère désormais dans un cadre élargi. Le développement des pratiques interdisciplinaires qui accompagne les recherches entreprises (collaborations entre météorologistes, épidémiologistes, médecins...) est une des causes du renouvellement des orientations et des discussions de la politique publique de réduction de la pollution atmosphérique. De 1994 jusqu'aux années 2000, cette interdisciplinarité s'est développée et approfondie mettant en relation de nombreuses institutions et administrations au plan local et européen.

\section{Conclusion}

Quelques conclusions se dégagent quant aux effets des études écologiques temporelles sur la requalification scientifique et politique de la pollution atmosphérique comme enjeu public. Elles ont incontestablement participé d'un renouveau des débats sur la manière de gérer la pollution atmosphérique en France. Si l'emploi de ces méthodologies a suscité des polémiques et cristallisé des oppositions entre les épidémiologistes classiques et ceux qui 
développent de nouvelles approches, on assiste aujourd'hui à un développement de l'épidémiologie environnementale dans la diversité de ses méthodes. Les études de cohorte, les études transversales et de cas témoins se multiplient comme l'illustre l'un des derniers numéros d'Extrapol (2006, 29). L'utilisation de relations entre exposition et risque établies à partir de cohortes épidémiologiques menées aux États-Unis (KünzLI et al., 2000) pour évaluer les impacts sanitaires à long terme est un exemple parmi d'autres des effets de synergie provoqués par la diversité des méthodes employées par les épidémiologistes. Ces études, qui nécessitent souvent plus de temps et de moyens financiers que les études dites «écologiques», permettent d'engager de nouvelles discussions et de faire progresser l'état des connaissances. Des recherches sont entreprises sur les effets à long terme et les indicateurs sanitaires se diversifient. Aujourd'hui, les controverses ne peuvent plus s'interpréter comme la simple traduction d'oppositions disciplinaires ou méthodologiques, elles semblent revêtir les formes plus traditionnelles de la confrontation propre au champ scientifique. Et les interrogations sur la responsabilité sociale des épidémiologistes connaissent un regain d'intérêt, elles impliquent la communauté plurielle des épidémiologistes et plus largement ceux qui travaillent dans le champ de la santé publique et de l'environnement.

\section{Références bibliographiques}

BECK U. - 2001 - La société du risque. Éditions Aubier.

BERLIVET L., 2001 - « Déchiffrer la maladie». In Dozon J.-P., Fassin D. : Critique de la santé publique: une approche anthropologique, éditions Balland: 74-102 p.

BOUTARIC F., 2003 - Environnement et santé publique: Production d'un enjeu politique. Écologie et politique, $\mathrm{n}^{\circ} 27$ : 161-178; publié dans une version légèrement remaniée in Pollution Atmosphérique, $\mathrm{n}^{\circ} 177$ : 47-55.

Boutaric F., Lascoumes P., Rumpala Y., VAzeIlles I., 2002 - L'obligation d'information, instrument d'action publique. Rapport Cevipol-Ademe, $303 \mathrm{p}$. 
BOUTARIC F., LASCOUMES P., 2005 - «Les instruments d'action publique, vecteurs du changement: l'exemple des transformations de la politique de lutte contre la pollution atmosphérique (1961-1996)». Communication au colloque Concertation, Décision, Environnement organisé par le ministère de l'Écologie et du Développement durable et l'Inra 12 et 13 décembre à Toulouse.

Bouyer J., Hemon D., Cordier S., Derriennic F., Stucker l., Stengel B., Clavel J., 2001 - Épidémiologie: principes et méthodes quantitatives. Éditions Inserm.

CASSADOU S. et al, 2003 - Impact sanitaire de la pollution atmosphérique: des questions et des réponses (réponse à l'éditorial de Jacques Estève). Environnement, Risques et Santé, 2 : 85-88.

DAB W., 2006 - L'homme malade de l'environnement. Paris, Eska.

Dab W. Michelon T., Nicoulet I., 2006 - L'étude d'impact sanitaire: un outil de gestion des risques sanitaires liés à l'environnement. Responsabilité et Environnement, Annales des mines: 51-59.

DAB W., QUenel P., ZMirou D., 1996 - Santé et pollution urbaine. Natures, Sciences, Sociétés, $4: 4$.

DOZON J.P., FASSIN D., 2001 - Critique de la santé publique. Une approche anthropologique. Édition Balland, 362 p.

ESTÈVE J., 2003 - Combien de décès causés par la pollution atmosphérique? Au-delà de la polémique. Environnement, Risques et Santé, 2: 5-7.

Extrapol, $2006-\mathrm{n}^{\circ} 29$, Effets à long terme de la pollution atmosphérique: études européennes. Aussi in Pollution atmosphérique $n^{\circ} 190$.

FASSIN D., 2005 - Faire de la santé publique. Éditions de l'École nationale de la santé publique.

FEsty B., Quenel P., 2003 - Pollution atmosphérique et épidémiologie en France: une longue maturation. Pollution atmosphérique, numéro spécial: 23-34.

Gérin M., Gosselin P., Cordier S., Viau C., Quénel P., Dewailly E., 2003 - Environnement et Santé publique, fondements et pratiques. Edisem, diffusion Éditions Tec et Doc.

GOLDBERG M., 2001 - L'épidémiologie sans peine. Éditions Frison-Roche.

HILL A. B., 1965 - The environment and disease: association or causation? Proc. R. Soc. Med., 58 : 295-300.

Jollivet M., Legay J.-M., Megie G., 1996 - Propos d'étape. Natures, Sciences, Sociétés, 2, Éditorial.

Kunzli N. et al., 2000 - Public health impact of outdoor and trafficrelated air pollution: a European assessment. Lancet, 35 (9232): 795-801. 
LASCOUMES P., LE GALES P., 2004 - Gouverner par les instruments. Presses de sciences-Po.

Le Moullec Y., Medina S., Momas I., Pirard P., Quenel P., 1992 Évaluation des risques de la pollution urbaine sur la santé - Analyse des études épidémiologiques publiées entre 1980 et 1991. Rapport d'étude ORS Île-de-France, Paris, $300 \mathrm{p}$.

LEPAGE C., 1998 - On ne peut rien faire Madame le ministre... Paris, éditions Albin Michel.

Momas I., Pirard P., Quénel P., Médina S., Le Moullec Y., Dab W., FESTY B., 1993 - Pollution atmosphérique et mortalité : une synthèse des études épidémiologiques publiées entre 1980 et 1991. Revue d'Épidémiologie et de Santé Publique, 41 : 30-43.

PAARC, 1982 - Pollution atmosphérique et affections respiratoires ou à répétition. Bulletin européen de physiologie respiratoire, 18:87-99.

QueNEl P., ZMIROU D. et al., 1995 - Impact de la pollution atmosphérique urbaine de type acido-particulaire sur la mortalité quotidienne à Lyon et dans l'agglomération parisienne. Santé Publique, 4 : 363-376.

SCHWARTZ J., 1994 - Air pollution and daily mortality: a review and meta-analysis. Environmental research, $64: 36-52$.

SETBON M., 1996 - Le risque comme problème politique: sur les obstacles de nature politique au développement de la santé publique. Revue Française des Affaires Sociales, 50, 2 : 11-28.

VAZEILLES I., 2003 - La pollution atmosphérique: d'une controverse scientifique à une décision politique. Thèse de l'université de Paris-I, 529 p.

VLASSOPOULOU C., 1999 - La lutte contre la pollution atmosphérique urbaine en France et en Grèce. Définition des problèmes publics et changement de politique. Thèse de l'université de Pari- II, 645 p.

Vlassopoulou C., Lascoumes P., 1998 - Protéger l'air ou réguler les sources de pollution atmosphérique? Questions d'actualité, n 239 , Paris, La documentation française: 31-40. 



\title{
Attitudes et connaissances des médecins généralistes face aux risques environnementaux
}

\author{
Anne ATTANÉ, Françoise BOUCHAYER \\ Jean-Christophe MATTEİ, Katrin LANGEWIESCHE \\ Marc-Éric GRUÉNAIS
}

Les différentes catégories d'acteurs concernés, en France, par les risques sanitaires liés aux phénomènes environnementaux font l'objet, ces dernières années, d'un nombre croissant de recherches en sciences sociales. Parallèlement aux travaux consacrés aux groupes "profanes" (ANTOINE-PAILlE, 2002; ATTANÉ, 2002; NAHON, 1999 ; ZONABEND, 1993), l'attention des chercheurs s'oriente également vers les acteurs experts ou décideurs exerçant dans diverses instances en charge des questions de sécurité sanitaire et de santé des populations: gouvernement, administrations centrales, services déconcentrés régionaux ou départementaux, élus aux divers échelons territoriaux, municipalités, agences nationales - InVS, Afsse, Afssa, Afsset ${ }^{1}$ - etc (BESANÇON, 2004; BESANÇON et al., 2004; Chateauraynaud et TORny, 1999; ESTADEs et REMy, 2003; GILBERT, 2002; TORNY, 1997). L'approche en termes de logiques 
institutionnelles et de systèmes de gouvernance est cependant prépondérante dans ces travaux par rapport à celles en terme de positionnements ou de représentations des divers groupes d'acteurs professionnels. De plus, les médecins généralistes assurant des fonctions de clinicien en contact avec les patients n'ont, à ce jour, guère fait l'objet d'approches portant sur leurs perceptions des risques environnementaux et leurs conduites en la matière. C'est plus particulièrement à cette catégorie de protagonistes professionnels du champ de la santé que nous nous intéresserons ici².

Notre propos se centrera sur les attitudes et les sources d'informations des médecins généralistes exerçant en libéral. Les problèmes environnementaux considérés se rapportent principalement aux pollutions industrielles et aux nuisances produites par les grands équipements collectifs, notamment en milieu urbain et périurbain (trafic routier et aérien, traitement des déchets, ondes électromagnétiques, bruit, etc.). Ne sont donc pas compris dans notre champ d'étude les pathologies infectieuses «importées» (grippe aviaire, SRAS, paludisme, etc.), les effets sanitaires des manifestations climatiques (canicule, réchauffement de la planète...) ni tout le domaine des troubles et des maladies découlant de l'environnement en milieu de travail. Le matériau sur lequel se fonde notre réflexion a été recueilli dans le cadre d'une étude exploratoire portant sur les perceptions des risques environnementaux (ATTANÉ et al., 2005). Ce travail a notamment donné lieu à la réalisation d'une série d'entretiens qualitatifs conduits auprès de médecins généralistes exerçant dans deux sites des Bouches-du-Rhône (Marseille et Fos-sur-Mer) et à l'analyse de quatre supports de presse professionnels destinés à un lectorat de praticiens généralistes: La revue du praticien (série monographie et série médecine générale), Le concours médical et Le quotidien du médecin. Nous présenterons successivement le contenu et les apports de ces deux corpus de données avant de faire le point sur l'émergence actuelle de la

\footnotetext{
$\mathbf{2}$ Le présent texte reprend des informations réunies dans le cadre du projet «Perceptions des risques environnementaux (Marseille et alentours). Etude exploratoire en sciences sociales auprès des médecins et dans la presse » (IRDShadyc), étude réalisée pour le compte de l'Observatoire euro-méditerranéen Environnement et Santé, Direction de la qualité de vie partagée, ville de Marseille.
} 
médecine environnementale, en France. Puis nous terminerons en proposant quelques pistes de réflexion se rapportant aux modalités de constitution des savoirs des médecins généralistes en regard de l'hétérogénéité des ensembles disciplinaires auxquels ils se réfèrent (l'épidémiologie et la recherche médicale notamment).

\section{Des médecins diversement informés et concernés}

Deux postulats de départ ont guidé nos questionnements de recherche et orienté le travail de terrain en direction des médecins généralistes. Tout d'abord ces praticiens peuvent tenir, sur les questions environnement/santé, un rôle de médiateurs légitimes auprès de la population. Face à certaines catégories de personnes - asthmatiques, insuffisants respiratoires, personnes âgées, parents de jeunes enfants, etc. - ou en réponse aux questions posées par tout type de patient, ces professionnels sont amenés à conseiller, à informer, à prévenir et éventuellement à décider de prescriptions spécifiques. En second lieu, les médecins généralistes peuvent être considérés comme des acteurs hybrides. Ils disposent d'une certaine expertise sur les questions relatives au maintien de la santé et à la pathogenèse tout en n'étant pas eux-mêmes des spécialistes des risques environnementaux et de leurs effets cliniques et physiologiques (ils ne sont pas toxicologues, ni épidémiologistes, etc.). En outre, ils sont également des citoyens plus ou moins sensibilisés aux problèmes de pollution, de qualité et de cadre de vie, etc. Considérant le caractère composite des rôles et des statuts professionnels et civiques de ces praticiens, nous avons cherché à connaître plus précisément ce qu'il en était de leurs perceptions des risques environnementaux, dans quelle mesure ils se trouvaient confrontés, dans leur pratique quotidienne, à ces questions, comment ils s'en débrouillaient, et quels étaient leurs degrés et leurs sources d'information.

Nous voulions en outre tester l'hypothèse d'une éventuelle incidence du lieu d'implantation de leur cabinet - et donc du cadre de vie de leur patientèle et souvent aussi du leur - sur leurs attitudes 
à l'égard des risques sanitaires, en général et en référence aux situations environnementales locales. Aussi avons-nous pris le parti de conduire les entretiens auprès de médecins installés dans deux sites différents: à Marseille où douze praticiens ont été interviewés (dix dans le centre-ville et deux dans la vallée de l'Huveaune) et à Fos-sur-Mer où nous avons rencontré cinq autres praticiens. Si aucun de ces sites n'est réputé pour la qualité de son environnement, notamment en ce qui concerne la pollution atmosphérique et sonore, le site de Fos-sur-Mer, et, dans une moindre mesure, celui de la vallée de l'Huveaune (située à la périphérie sud-est de Marseille) présentent des taux de pollution et des cumuls de nuisances particulièrement élevés liés à l'implantation d'industries lourdes (métallurgie, pétrochimie notamment) et de grands équipements (port pétrolier, autoroutes, aéroport).

Les investigations réalisées relèvent donc d'une démarche qualitative visant à disposer d'un tableau de la diversité des perceptions et des conduites vis-à-vis des risques environnementaux et non d'une représentativité des opinions référée à un échantillon de praticiens qui serait lui-même à l'image de l'ensemble de la profession. Trois principaux ordres de résultats se dégagent de l'analyse des entretiens. Ils se rapportent aux représentations sur les liens entre environnement et santé, aux effets de la proximité de sites à risques et au degré d'information des médecins sur ces questions.

\section{Un fond commun de représentations}

Les troubles ou les pathologies perçus comme liés aux problèmes environnementaux sont les mêmes pour l'ensemble des médecins rencontrés. Tous mentionnent en premier lieu les maladies respiratoires, l'asthme et les allergies. En second lieu, ils insistent sur la nervosité et le stress générés par les nuisances sonores ou par l'angoisse de devoir vivre avec une pollution de plus en plus importante: "Il y a des stress physiques effectivement; mais la peur de respirer quelque chose de pollué est un stress aussi et ça se transmet au niveau respiratoire», souligne un des médecins rencontrés. Tous s'accordent sur le fait que les enfants sont les premiers touchés par la pollution et que le nombre de maladies respiratoires imputées à la pollution de l'air tend à augmenter. 
Parmi tous les médecins interrogés, autant à Marseille qu'à Fos, la même hiérarchisation des problèmes environnementaux se retrouve. Le problème environnemental perçu comme le plus important pour eux-mêmes et pour leurs patients est celui de la pollution atmosphérique due aux industries et aux voitures, suivi par le bruit et par les ondes électromagnétiques. Plusieurs praticiens ont évoqué que leur appréciation in situ de la pollution se fonde souvent sur la perception sensorielle qu'ils peuvent en avoir par la vue, l'odorat et l'ouie. Ainsi, les médecins interviewés dans la vallée de l'Huveaune mentionnent la piètre apparence de l'eau de la rivière, révélatrice de la présence de substances toxiques et sales rejetées par les riverains autant que par les industries. Mais ces médecins font également part de leurs réserves quant à la validité de ce mode d'appréciation: «D'un point de vue scientifique, aboutirait-on au constat qu'effectivement il y a pollution lorsqu'on la sent? "Ils prennent également soin de préciser que s'ils peuvent déceler une pollution qui se sent ou se voit, il se peut très bien aussi qu'ils ne se rendent pas compte par eux-mêmes des diverses formes de pollution. On note que ne sont quasiment pas évoqués certains risques comme la contamination des aliments par les pesticides ou encore la pollution intérieure de l'habitat par les produits qui s'y trouvent (mobilier aggloméré, revêtements de sol, etc.) ou y sont utilisés (colles à usages divers, produits d'entretien ménager, etc.). Il en est de même des dangers potentiels en débat actuellement comme les installations nucléaires ou encore les OGM.

Parallèlement, on observe parmi les praticiens interviewés, des stratégies d'euphémisation analogues à celles décrites dans la littérature à propos des populations riveraines (ATTANÉ, 2002; ZONABEND, 1993): plus une personne est exposée à des risques, plus elle essaie de les minimiser afin de supporter le stress psychologique que l'exposition à ces dangers réels ou potentiels représente. Ainsi, un médecin de la vallée de l'Huveaune déclare "ça me stresse le bruit! Parfois je n'en peux plus! Entre les voitures, les usines de l'autre côté, les klaxons... Les gens se plaignent d'ailleurs, et pourtant on n'est pas au centre-ville. Là-bas, c'est pire encore!". Un praticien de Fos-sur-Mer estime que "l'automobile est vraiment très polluante, et d'ailleurs la ville de Marseille est bien plus polluée que la ville de Fos». Un interlocuteur 
du centre de Marseille remarque de son côté que «la pollution augmente, mais nous ne sommes pas dans un quartier très pollué ici. Les plus pollués ce sont le $11^{\mathrm{e}}$, le Vieux-Port et l'étang de Berre; c'est connu!». Chacun attribue à ceux vivant dans un «ailleurs», même relativement peu éloigné, une exposition à une pollution plus importante et plus nocive.

\section{Les effets de la proximité aux sites pollués ou à risques}

Sur toute une palette de questions, les médecins marseillais et ceux de Fos-sur-Mer ont tenu des propos sensiblement différents. En premier lieu, les médecins exerçant à Fos parlent tous spontanément du projet d'installation à proximité d'une usine d'incinération des déchets domestiques en provenance de la ville de Marseille. À côté des arguments sanitaires mettant notamment en avant le risque de contamination du lait maternel par la dioxine rejetée par la combustion, les médecins rencontrés à Fos font état de patients qui ont mis leur maison en vente pour quitter définitivement la région à cause d'un surplus de pollution qu'ils jugent intenable. Certains envisagent d'ailleurs de prendre pour eux-mêmes une telle décision. Les médecins interrogés à Fos déclarent en outre que la question de l'incinérateur revient fréquemment dans les discussions avec leurs patients. D'une manière générale, les médecins de Fos se sentent particulièrement concernés par la proximité de nombreuses industries polluantes et expriment un sentiment très vif de saturation de l'espace par ce type d'installation et par les nuisances qui en découlent: «La vie ici risque de pâtir de l'inconséquence des pouvoirs publics. Il faut que ceux-ci se rendent compte que la population ne peut pas accepter ça en plus [l'incinérateur], c'est trop dans une zone déjà industrielle!" Les médecins qui ont spontanément tenu divers propos sur les inégalités sociales face aux expositions à des risques sanitaires environnementaux se retrouvent en outre préférentiellement parmi ceux exerçant à Fos-sur-Mer.

À Marseille en revanche, aucun des praticiens rencontrés n'a évoqué spontanément l'usine d'incinération d'ordures ménagères dont la construction est prévue dans la commune de Fos. Ce constat, là encore, peut être rapproché de phénomènes plus 
généraux comme le syndrome $\mathrm{NIMBY}^{3}$ dont rend compte la littérature pour désigner l'opposition des associations ou des populations locales à un projet jugé à risque et qui pourtant répondrait à un besoin collectif. Plus les personnes vivent proches du lieu envisagé pour l'implantation d'un équipement estimé dangereux, plus elles se sentent concernées et se mobilisent en conséquence. Tandis qu'aucun des médecins de Marseille ne s'est dit adhérent à une organisation de défense de l'environnement, plusieurs médecins interrogés à Fos se sont engagés dans la vie politique locale (en tant qu'adjoint au maire, par exemple), ou dans des associations pour la défense de leur environnement et contre l'installation de l'incinérateur.

Enfin, on note que la notion d'environnement évoque, pour les praticiens marseillais, aussi bien les équipements de type industriel implantés dans l'espace local que l'habitat ou l'environnement social et professionnel. Ceux-ci mentionnent en effet aussi fréquemment le tabagisme, l'hygiène alimentaire dans les restaurants, la surcharge en informations et en publicité, le stress au travail, les déjections des chiens sur les trottoirs que la pollution de l'air ou les nuisances par le bruit. En revanche, les réponses des médecins de Fos témoignent d'une acception de l'environnement d'ordre principalement écologique. Ils mettent surtout en avant la pollution de l'air par les industries et les automobiles, les nuisances par le bruit des avions et des autoroutes ainsi que la dégradation de la végétation.

\section{Une information estimée insuffisante et peu fiable}

D’une manière générale, les médecins insistent sur les difficultés d'établir des liens sûrs et scientifiquement établis entre certains symptômes ou certaines maladies et des situations environnementales, notamment du fait de la multiplicité des facteurs à considérer. Les études épidémiologiques ou toxicologiques leur semblent le seul moyen légitime qui permettrait de se prononcer sur les liens entre risques sanitaires et pollution. Tous les interlocuteurs rencontrés estiment qu'ils ne disposent pas de suffisamment d'informations

| 3 NIMBY = Not In My BackYard, c'est-à-dire "pas dans ma cour ». 
solides sur ces questions. Ils expriment en outre leurs doutes quant à l'objectivité et la provenance de celles auxquelles ils ont accès (par les médias généralistes notamment) et ont le sentiment d'être dépendants de la propagande politique, ou de rumeurs scientifiquement infondées. Ils s'accordent également sur le fait que les risques sanitaires liés aux problèmes environnementaux sont très peu traités dans la presse médicale, qu'il s'agisse de la problématique des déchets ou d'autres questions environnementales.

Les revues médicales les plus lues sont Le quotidien du médecin, suivi par La revue du praticien. On note toutefois que les médecins de Fos paraissent mieux informés que leurs collègues marseillais. Ils évoquent les informations reçues par Airfobep, par la Drire ${ }^{4}$, ou obtenues par la recherche personnelle d'informations sur Internet. Les connaissances relatives au projet d'implantation d'un incinérateur d'ordures ménagères, au site de l'installation et aux procédures techniques sont chez eux plus précises que parmi leurs collègues marseillais. Mais en fin de compte, si les médecins de Fos ont davantage d'idées que leurs confrères de Marseille sur les débats techniques, ils n'ont guère plus d'éléments pour prendre position sur les grandes questions liées au traitement des déchets et ont l'impression d'être "dépassés". D’une manière générale, les référendums, les conseils de citoyens, les consultations publiques, etc., semblent appréciés par les médecins, mais ces mesures ne suffisent pas à dissiper un certain sentiment d'impuissance.

À la lumière des entretiens réalisés, les médecins apparaissent faiblement intéressés et impliqués par la problématique environnement/santé et ne se présentent pas comme initiateurs ou promoteurs d'informations ou d'actions sur les risques sanitaires liés à la pollution environnementale. Rares sont ceux qui défendent des opinions tranchées et méthodiquement argumentées dans ce champ. La plupart des médecins interviewés suivent plutôt une sensibilité environnementale que l'on rencontre dans la société globale. Dans la mesure où ils pourraient ne faire que renvoyer à leur clientèle leurs propres interrogations, on peut se demander si

4 Airfobep: association agréée pour la surveillance de la qualité de l'air (étang de Berre, ouest des Bouches-du-Rhône) ; Drire: Direction régionale de l'industrie et de la recherche. 
leur discours qui se présente comme une "parole autorisée", eu égard à leur statut de médecin, ne viendrait pas finalement conforter les inquiétudes de patients qui partagent les mêmes perceptions des risques, des incertitudes et du manque d'information.

Ces résultats peuvent être mis en regard de ceux de l'enquête par questionnaire conduite en 2005 auprès des médecins libéraux de Haute-Normandie, région qui se caractérise par une forte densité d'industries polluantes, notamment autour de l'estuaire de la Seine (Dupont et Suriré, 2005). Il ressort de cette étude que $47 \%$ des répondants estiment que les risques liés aux pollutions environnementales sont majeurs et avérés, $49 \%$ établissent un lien entre des pathologies fréquemment rencontrées et la pollution environnementale et $69 \%$ entre les pathologies environnementales et les inégalités sociales; $59 \%$ notent une augmentation des demandes des patients sur les causes environnementales des pathologies. Ces taux apparaissent plus élevés parmi les praticiens homéopathes ou acupuncteurs. L'enquête souligne en outre que les médecins se disent d'autant plus sensibilisés aux risques sanitaires environnementaux qu'ils exercent à proximité de sites à risques. Pour ce qui se rapporte à l'information dont ils disposent, $86 \%$ des enquêtés disent n'avoir reçu aucune formation en santé publique ou en santé environnementale (ce pourcentage apparaît moindre parmi les praticiens les plus jeunes) et $65 \%$ pensent ne pas apporter de réponses satisfaisantes aux questions posées par les patients sur les pathologies liées à l'environnement. Plus d'un tiers des médecins enquêtés sont demandeurs de formations continues ou d'une lettre ou revue sur les questions santé/environnement afin de combler leur déficit de formation mais aussi de mieux comprendre la situation actuelle marquée par un fort développement de technologies modernes dont il convient de mieux mesurer et analyser les effets sur la santé.

Au total, il ressort de ces deux études, qualitative et quantitative, que les médecins normands se sentent dans l'ensemble plus concernés que leurs collègues marseillais par les questions sanitaires environnementales, tandis que leur degré de sensibilisation s'apparente davantage à ceux de Fos-sur-Mer. Tous, quels que soit leur région et leur lieu d'implantation, font part de leur manque de formation et d'informations sur ces sujets. Dans les deux enquêtes, on retrouve des médecins exprimant un sentiment 
de vulnérabilité et d'impuissance face à la montée de pathologies problématiques corrélées aux évolutions de l'environnement.

Afin de prolonger les apports des enquêtes conduites auprès de médecins généralistes en exercice, nous avons cherché à apprécier dans quelle mesure et de quelles manières les liens entre environnement et santé se trouvaient traités dans la presse professionnelle destinée à ces praticiens.

\section{Le risque environnemental dans la presse médicale généraliste}

La presse médicale représente une source d'information importante pour les médecins généralistes. L'analyse que nous avons conduite s'est portée sur trois revues cliniciennes de référence: La revue $d u$ praticien série Monographie (bi-mensuelle) et série Médecine générale (hebdomadaire) et Le concours médical (hebdomadaire) - ainsi que sur Le quotidien du médecin (qui paraît cinq jours par semaine). Les versions papier des revues ont été exhaustivement étudiées, ce qui a permis d'y repérer des articles courts ou des informations brèves qui ne sont pas listées dans le sommaire ni référencées sur leurs sites Internet (peu propices, dans leur configuration actuelle, à ce type d'usage) tandis que l'exploitation du quotidien du médecin a pu être réalisée à partir de son site Internet. La période étudiée va de janvier 2003 à mars 2005 (et juin 2005 pour Le quotidien du médecin), soit une durée d'un peu plus de deux ans. Les résultats des investigations conduites sont présentés successivement pour chacun des deux types de presse professionnelle. Puis nous verrons de quelle manière le rôle du médecin praticien sur les questions santé/environnement est abordé dans l'ensemble de ce corpus.

\section{Les revues cliniques}

Pour ces revues, un premier repérage nous a amenés à élargir le champ considéré au-delà des questions relatives à la pollution de type industriel. On repère en effet dans les trois séries étudiées un 
recours relativement fréquent, dans le corps des articles portant sur différentes pathologies (cardiaques, rhumatismales, cancéreuses, psychiatriques, etc.), à des termes comme «facteurs environnementaux " ou "éléments liés à l'environnement"; ces formulations recouvrent une gamme de facteurs allant de l'alimentation, de l'alcool et du tabac au stress en passant par la présence de produits toxiques dans les milieux de vie domestique ou professionnel, etc. On note également dans ces revues la présence régulière d'articles traitant de l'environnement en milieu de travail, des facteurs climatiques, de la iatrogénie et des infections nosocomiales, etc. Nous avons donc pris le parti de comptabiliser et de sérier dans un premier temps l'ensemble de ces manières d'approcher les questions environnementales pour nous centrer dans un second temps sur l'analyse des articles se référant plus particulièrement à la pollution et aux nuisances découlant de certains grands équipements collectifs ou dispositifs technologiques.

Au total, 62 articles ont été repérés. Ils sont de format très variable - allant de plusieurs pages à, le plus souvent, une ou une demi-page - et ne représentent qu'une infime partie de l'ensemble des textes publiés sur la période étudiée (moins d'un article pour quatre numéros de revue). Nous les avons ordonnés en huit catégories définies en fonction du type de problème environnemental abordé et du statut occupé par la question environnementale dans l'article (thème principal repérable dès le titre ou dimension tangentiellement évoquée dans le corps du texte). La répartition ainsi établie est la suivante (chaque article a été répertorié dans une seule catégorie, même si quelques-uns pouvaient relever d'un double ou d'un triple classement) (cf. tableau en annexe).

Deux principaux enseignements peuvent être retirés de cette répartition. Tout d'abord, deux articles sur trois renvoient à des risques environnementaux non référés à la pollution ou aux technologies en milieu ordinaire (soit 41 articles sur 62). Neuf articles se rapportent aux facteurs climatiques ou naturels, 8 aux pathologies professionnelles, 6 à la médecine tropicale, $8(3+5)$ à des questions liées à l'environnement médico-sanitaire (dont 3 à la iatrogénie); dans 10 articles enfin, les facteurs environnementaux sont simplement évoqués dans le cours du texte. Ce traitement élargi de la notion d'environnement rappelle que la médecine dite environnementale relève d'une longue histoire et n'a pas 
attendu les risques liés à la vie dans les sociétés industrielles et «technologisées» d'aujourd'hui pour se constituer (courant hygiéniste, médecine tropicale, santé publique, etc.).

On remarque ensuite que seulement 21 articles, soit un tiers de la totalité de ceux repérés, traitent de questions de type pollution industrielle $(n=7)$ ou de risques technologiques divers $(n=14)$. Plus précisément, ces 21 articles abordent les sujets suivants (voir en annexe la liste et les intitulés de ces articles):

- pollution atmosphérique ( $n=4)$;

- pollution des sols ou de l'air par les dioxines issues de l'incinération des déchets $(n=3)$;

- lignes à moyenne ou haute tension et ondes électromagnétiques ( $\mathrm{n}=5$, dont 2 sur les risques liés aux éoliennes);

- autres produits toxiques ou infectieux $(n=5)$ : plomb, vaisselle peinte, herbicides, oxyde de carbone, qualité de l'eau;

- divers $(n=4)$ : irradiations naturelles ou artificielles (radiothérapie), bioterrorisme, bruit, bacs à sable.

Certains risques pourtant avérés ne font l'objet d'aucun article dans le corpus étudié. Tel est le cas pour le risque nucléaire (centrales, déchets) ou encore les pesticides (l'article sur les herbicides classé dans la rubrique « divers» traite des produits utilisés à l'échelon domestique pour les potagers).

Ces 21 articles sont le plus souvent ciblés sur des questions précises et destinés à faire le point sur l'état des connaissances ou des méconnaissances scientifiques concernant la question traitée. Les quelques approches relevant d'une vision plus synthétique de ces problèmes sont le fait d'articles prenant la forme d'une interview d'un expert, chercheur et (ou) représentant d'une institution (Inserm, Direction générale de la santé, InVS, etc.). Enfin, les sujets environnementaux sont apparus sensiblement plus souvent abordés dans Le concours médical ( $10+4=14$ articles sur 21) que dans les deux séries de La revue du praticien $(2+2+1+2=$ 7 articles sur 21).

D'une manière générale, on note une attitude rédactionnelle extrêmement prudente quant à la validation des liens entre certains facteurs environnementaux et des manifestations pathologiques. 
Les références aux études existantes sur ces questions soulignent fréquemment les limites avec lesquelles des résultats positifs doivent être considérés du fait de biais méthodologiques, de facteurs de confusion, etc. Ce type de vigilance apparaît particulièrement de mise à propos des liens entre pollution et cancers. Cette posture générale se trouve toutefois contrebalancée dans les articles fondés sur des interviews de responsables d'agences ou de grands programmes nationaux qui font valoir la diversité et l'amplitude des dangers sanitaires liés aux pollutions environnementales et qui soulignent la nécessité de développer des recherches sur ces questions. Tel est le cas du texte intitulé «Pollution atmosphérique: quel impact sanitaire? » qui présente les premiers résultats d'une étude épidémiologique nationale conduite dans neuf villes françaises (EILSTEIN et al., 2004); cet article souligne, en conclusion: «De plus, lorsque l'effet à long terme est pris en compte, les nombres de cas attribuables [à la pollution atmosphérique] deviennent comparables à ceux du tabac ». Les enjeux relatifs à l'allocation de fonds pour la recherche et à la reconnaissance institutionnelle qui s'y trouve liée contribuent semble-t-il à atténuer les précautions méthodologico-scientifiques (et politiques) habituellement mises en avant. Le caractère en partie disparate des orientations éditoriales des revues analysées est sans doute également attribuable à la superposition, dans la période étudiée, d'un ancien et d'un nouveau régime de traitement de ces questions correspondant respectivement à la période antérieure versus la période postérieure au lancement des grands programmes nationaux, notamment le Programme national santé environnement, 2004-2008 (PNSE ${ }^{5}$ ).

\section{Le quotidien du médecin}

Une première investigation faite sur le site internet de ce quotidien, très largement diffusé parmi les médecins généralistes, a d'emblée révélé que les questions relatives à l'environnement, et plus précisément celles se rapportant à la pollution de type industriel et

5 Le PNSE se décline en programmes régionaux et comporte deux grands volets: santé/travail et santé/environnement. II est piloté par quatre ministères, en charge respectivement de la Santé, de l'Écologie et du Développement durable, de la Recherche et du Travail. 
aux risques technologiques étaient fréquemment abordées. Après divers essais visant à cibler au mieux les mots clés à retenir afin de collecter l'information recherchée sans être noyé par son abondance ou son hétérogénéité nous avons retenu l'interrogation "Pollution et santé» qui a drainé 113 références pour la période s'étalant du

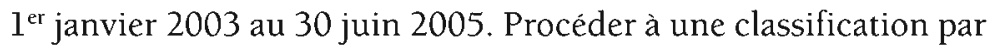
sujet traité de cet ensemble d'articles est vite apparu délicat du fait de la grande diversité des domaines abordés, de la pluralité des entrées pour un même sujet (par exemple pour l'eau: les inondations, la baignade, la qualité et le contrôle de l'eau distribuée, la pollution des nappes phréatiques, les réglementations en vigueur, les instances concernées, les «affaires » locales, etc.) et du décalage existant parfois entre le titre et le contenu de l'article. Un classement analogue à celui réalisé pour les revues cliniciennes aurait été en partie arbitraire et de ce fait peu satisfaisant. Aussi avons-nous pris le parti de nous axer sur une approche transversale du style et des contenus rédactionnels de ces 113 articles.

Il apparaît donc en premier lieu que Le quotidien du médecin a développé une approche des questions de santé et pollution diversifiée et largement associée à l'actualité scientifique et institutionnelle (études épidémiologiques ou programmes gouvernementaux) mais aussi à l'actualité sociale et politique dans le domaine médicosanitaire: recours d'élus locaux pour non-respect de normes environnementales, mobilisation de diverses catégories d'acteurs, conflits ou controverses, débats de société, etc. Les questions posées par les risques et les déchets nucléaires y sont fréquemment abordées. Ce quotidien a en outre instauré, depuis 1993, les «trophées Épidaure " récompensant chaque année un scientifique, un jeune chercheur et un praticien pour des recherches contribuant à «mieux comprendre et mieux prendre en compte les liens existant entre environnement et santé $»$. Il existe donc une orientation éditoriale affichée dans Le quotidien du médecin sur les questions médico-sanitaires d'ordre environnemental.

Le traitement formel des sujets environnementaux abordés dans ce quotidien (comme de tous les autres sujets) apparait davantage journalistique que dans les revues cliniciennes. Cela se repère notamment dans les titres des articles fréquemment empreints d'une tonalité «accrocheuse» ou sensationnaliste, par exemple 
Les particules envahissent l'air du métro et du RER (17 juillet 2003), La pollution atmosphérique accusée dans le cancer bronchique (5 septembre 2003), Un combat sans fin pour le lait pur (25 mars 2004). Toutefois, l'impression alarmiste alimentée par ces intitulés se trouve en général tempérée par les contenus des articles qui comportent le plus souvent une information et une argumentation rigoureuses. Tel est particulièrement le cas des nombreux articles rapportant l'interview d'un expert ou d'un responsable institutionnel.

\section{Le rôle du médecin généraliste}

La question des conduites et du rôle que peuvent avoir les médecins généralistes en matière de risques sanitaires environnementaux ne se trouve que peu abordée dans La revue du praticien ou Le concours médical, qu'il s'agisse des aspects cliniques de leur pratique (par exemple à propos de certaines catégories de patients vulnérables) ou de leur rôle de référent en matière de santé des populations, à l'échelon local ou national. Sur les 62 articles traitant des questions santé/environnement (voir le tableau en annexe) nous en avons repéré six, courts pour la plupart, abordant ces aspects. Deux se rapportent à la conduite à tenir et à l'information à avoir face aux risques de pollution liés à un incinérateur local ; il s'agit d'articles courts publiés dans une rubrique du Concours médical intitulée "Consultations » et dédiée aux questions des lecteurs (GARNIER, 2003 et 2004). Deux autres articles sont consacrés au rôle du médecin en cas d'accident collectif, d'attentat ou de catastrophe naturelle (AUBERT 2003; MARÉCHAUX, 2004). Un article enfin porte sur "La chambre de l'allergique»; il souligne le rôle du médecin en direction des patients asthmatiques et met en exergue la création récente d'une nouvelle orientation professionnelle, celle de "conseiller médical en environnement intérieur" (DE BLAY et LIEUTIER-COLAS, 2004). Enfin, un article consacré à l'actualité médicale dans les agences sanitaires pose, dans son dernier paragraphe intitulé "En pratique», la question de l'attitude du médecin généraliste vis-à-vis des inquiétudes et des interrogations de ses patients en ces termes: "Pour répondre aux patients, le plus simple est de prendre rapidement connaissance de l'actualité médicale «médiatique» (par la radio, la télévision ou la presse quotidienne) 
et de vérifier sur les sites ad hoc la réalité de l'information. Le tout ne doit pas demander plus de 10 minutes par jour» (EvEILLARD, 2004). Au-delà de leur simplicité et de leur clarté, ces conseils laissent entrevoir toute la complexité du rôle de conseiller bien informé - sur des sujets pleins d'incertitudes - des médecins au contact avec les patients.

Dans Le quotidien du médecin, le rôle du généraliste sur les questions santé/environnement apparaît un peu plus fréquemment abordé. On repère régulièrement dans ce journal des articles faisant état des campagnes de sensibilisation menées par les pouvoirs publics en direction des praticiens de ville, comme dans les deux articles Les médecins franciliens, futurs remparts contre la pollution? (19 juin 2003) et Des médecins pionniers du combat environnemental (29 mars 2004). Un autre article Les médecins appelés à la rescousse (17 mars 2005) relate les difficultés rencontrées sur certains sites pour trouver des personnes volontaires pour participer à une enquête épidémiologique sur les incinérateurs et l'appel lancé par les initiateurs de l'étude auprès des généralistes locaux pour sensibiliser leurs patients à l'intérêt de ce type de recherche. D'autres articles rendent compte de l'engagement de médecins dans des mobilisations de nature militante en faveur du « combat environnemental», notamment à propos des incinérateurs d'ordures ménagères.

Au final, il apparaît que si Le quotidien du médecin aborde un éventail ouvert de questions traitant des liens entre environnement et santé, les revues cliniciennes ont une posture éditoriale nettement plus réservée en la matière. L'ensemble de ces supports de presse médicale ne livre guère d'informations, de conseils ou d'expériences sur les conduites pouvant être adoptées face aux risques environnementaux et ne constitue pas, pour les médecins et pour la période étudiée, un cadre méthodiquement documenté de références pour l'action. Nous faisons pour notre part l'hypothèse que la montée en légitimité institutionnelle, politique et scientifique, à l'échelon national, des questions qui touchent aux relations entre environnement et santé donnera progressivement lieu au développement multiforme d'un intérêt du corps médical pour ces problèmes. Sans doute alors la presse professionnelle en rendra-t-elle davantage compte et entretiendra par là le mouvement 
de construction progressive d'une médecine environnementale en prise sur les évolutions actuelles, et à venir. C'est une évolution de cet ordre qu'illustrent certains faits comme le développement, déjà évoqué, d'une "spécialisation médicale " en environnement intérieur orientée plus particulièrement vers les personnes allergiques ou asthmatiques ou comme la tenue en 2005 à l'initiative de l'Union régionale des médecins libéraux de Haute-Normandie du $1^{\text {er }}$ congrès national sur le thème «Médecine libérale et pathologies environnementales » (Rouen, 7 et 8 octobre 2005).

Fin 2005, La revue du praticien, en collaboration avec la Direction générale de la santé, a consacré un numéro au médecin face aux risques sanitaires et environnementaux (t. $55, \mathrm{n}^{\circ} 18,30$ novembre 2005). La première partie de ce document est consacrée à la présentation du dispositif français de sécurité sanitaire (agences, institutions, plans de mobilisation collective, dispositif de surveillance, etc.); puis sont exposés divers exemples de gestion des risques parmi lesquels la pollution de type industriel ou technologique apparaît peu abordée (un article porte sur la téléphonie mobile), les pathologies infectieuses importées, les intoxications alimentaires ou la iatrogénie l'étant davantage. Si, en toute logique, l'éditorial de ce numéro indique que le bon fonctionnement de ce dispositif «nécessite que les médecins y participent activement», il souligne également le fait que «l'action en faveur de la santé publique passe aussi par des prises de position dénonçant certains lobbies". Sont dans cette optique désignés de manière circonstanciée les manœuvres des industriels de l'amiante ou du tabac et les groupes de pressions liés à la viticulture (exploitants et élus). Lors d'échanges informels que nous avons eus avec des représentants de la profession (Conseil de l'ordre, spécialistes hospitalo-universitaires) ou dans les propos tenus par nombre d'intervenants au colloque précité, des prises de position analogues ont également été exprimées avec vivacité (en direction de l'industrie lourde ou agro-alimentaire notamment).

Divers autres indices viennent alimenter le diagnostic de l'émergence, en France, d'une médecine environnementale intégrant à son champ de connaissances et d'action les composantes de la situation propres au temps présent, en particulier pour tout ce qui concerne les pollutions de type industriel ou liées à de grands 
équipements technologiques. Jusqu'à une date récente, les quelques ouvrages à destination des médecins praticiens disponibles en français étaient des traductions de livres provenant de l'étranger (voir par exemple REIHL, 2002, traduit de l'allemand). L'ouvrage de référence actuel directement rédigé en français est le fruit du travail d'un collectif pluridisciplinaire de chercheurs francoquébécois (GÉRIN et al., 2003). Parallèlement, on observe la création de quelques réseaux de santé dédiés à la prise en charge de pathologies aggravées par des facteurs environnementaux (par exemple, pollution et habitat, pollution atmosphérique et patients coronariens), ou encore le développement de modules de formation médicale (initiale ou continue) en santé environnementale. On peut en outre penser que, dans un proche avenir, la médecine environnementale constituera, pour les médecins praticiens et selon des voies qui se définiront chemin faisant, un créneau de "spécialisation" et d'élaboration d'identités professionnelles au même titre que d'autres orientations ou modes d'exercice particulier (BOUCHAYER, 1994; ROBELET, 1999).

En guise de conclusion et d'ouverture pour la réflexion, nous voudrions souligner quelques caractéristiques actuelles du domaine médico-scientifique s'intéressant aux risques sanitaires environnementaux relatifs notamment à la pollution de type industriel. Ce domaine représente en effet, pour les médecins généralistes, l'espace de connaissances et de référentiels pour l'action à partir duquel se forge leur propre expertise et nous avons vu que, pour l'heure, nombre de médecins généralistes se disent insuffisamment informés et peu à l'aise pour composer avec des informations dont ils doutent parfois de la fiabilité.

\section{Des savoirs marqués par des incertitudes et des controverses}

L'évolution des connaissances et le caractère fluctuant des «conduites à tenir » traversent, à chaque époque, l'ensemble du champ de la médecine. Certaines de ses spécialités ou de ses branches paraissent cependant, à certaines périodes, particulièrement marquées, voire 
structurées par des incertitudes et des controverses. Tel est actuellement le cas des pathologies environnementales (voir par exemple DAB, 2006; DAB et al., 1998). On repère, en outre, dans ce champ des positionnements d'ordre politique contrastés, portés par les producteurs «à la source » de savoirs en la matière: médecins spécialistes hospitalo-universitaires (pédiatres, endocrinologues, allergologues, pneumologues, cancérologues, etc.), épidémiologistes, fondamentalistes (toxicologues notamment). Schématiquement, trois types de postures se trouvent exprimés.

Certains affirment l'existence de corrélations massives et à grande échelle entre des données environnementales relatives à l'utilisation généralisée de certains produits (pesticides, engrais, dérivés du pétrole, etc.) et au développement des technologies (champs électromagnétiques, pollution industrielle, etc.). Un des chefs de file de cette posture est le Dr Dominique Belpomme, cancérologue et auteur de plusieurs ouvrages à destination du grand public (voir notamment BELPOMME, 2004). Les thèses développées par ce chercheur sont vivement critiquées par toute une partie du milieu médico-scientifique et institutionnel engagé, à différents titres, dans la production de savoirs et d'études sur ces questions. D'autres, tout en reconnaissant que l'état des connaissances ne permet pas actuellement d'apporter strictement la preuve de ces corrélations ou des systèmes de causalités qui les sous-tendent, se positionnent en "énonceurs de dangers" et cherchent activement à mobiliser les pouvoirs publics et les décideurs. Ainsi, un pédiatre endocrinologue ayant mené des travaux sur l'augmentation des malformations congénitales sexuelles chez les nouveau-nés attribuables à l'usage d'engrais ou de pesticides souligne: "Attendre d'en savoir assez pour agir en toute lumière, c'est se condamner à l'inaction ${ }^{6}$. D'autres enfin, notamment parmi les chercheurs «fondamentalistes» et en épidémiologie, insistent sur le fait qu'il est impératif d'en savoir davantage et de lever les incertitudes par le développement de recherches avant de se prononcer sur des dangers qui, estiment-ils, restent à ce jour supposés, et donc avant de s'engager dans des mesures ciblées de réglementation et de prévention (c'est une attitude de cet ordre qui 
est apparue prépondérante dans les revues cliniciennes étudiées). Au-delà des incertitudes et des désaccords, assez habituels dans le champ de la connaissance et de la recherche scientifiques, c'est donc aussi sur le registre des jeux d'acteurs et de leur positionnement en matière d'art de gouverner et de décider que s'expriment ces controverses. Les sciences sociales, en particulier la sociologie et les sciences politiques, se sont pour leur part abondamment saisies, ces dernières années, de ces thématiques (voir par exemple BARTHE, 2006; Dourlens, 2003; Dumoulin et al., 2005; Jouzel et LANDEL, 2005; VAILlancourt et COTNOIR, 2003). Se pose alors la question de savoir comment évolueront à l'avenir les attitudes et les positionnements professionnels des praticiens de base de la médecine et de leurs représentants.

\section{Références bibliographiques}

ANTOINE-PAille M.-T., 2002 - «La perception des risques santé/environnement par le public: un modèle et son application au domaine de l'eau ». In Giroult E. Seux R. (éd.): Eau, Environment, Santé. Un enjeu majeur pour le XXIe siècle, Rennes, éditions ENSP: 195-203.

ATTANÉ A., 2002 - Pêcher, courir, trier ses déchets... pratiques de l'environnement ou rapport aux autres? Europea, 1-2: 261-282.

Attané A., Bouchayer F., Gruénais M.-E., Langewiesche K., MATTEİ J.-C., 2005 - Perceptions des risques environnementaux (Marseille et alentours). Étude exploratoire en sciences sociales, auprès des médecins et dans la presse. IRD UR 002, Shadyc Ehess-Cnrs, ville de Marseille, direction de la qualité de vie partagée, rapport de recherche, $110 \mathrm{p}$.

AUBERT J.-P., 2003 - Risque bioterroriste: les généralistes en $1^{\text {re }}$ ligne? La revue du praticien-Médecine générale, t. 17, nº 604: 269.

BARTHE Y., 2006 - Le pouvoir d'indécision. La mise en politique des déchets nucléaires. Paris, Economica.

Belpomme D., 2004 - Ces maladies créees par l'homme. Comment l'environnement met en péril notre santé. Paris, Albin Michel.

BESANÇON J., 2004 - Les agences de sécurité sanitaire en France. Revue de littérature commentée. Les cahiers du GIS, Risques collectifs et situations de crise, $n^{\circ} 2$, CNRS-MSH-Alpes. 
Besançon J., Borraz O., GRANDClement-ChafFy C., 2004 - La sécurité alimentaire en crise. Les crises Coca-Cola et listeria de 1999-2000. Paris, L'Harmattan.

BOUCHAYER F., 1994 - «Les voies du réenchantement professionnel ». In Aiach P., Fassin D. (éd.): Les métiers de la santé. Enjeux de pouvoir et quête de légitimité, Paris, Anthropos: 201-225.

Chateauraynaud F., TORNY D., 1999 - Les sombres précurseurs. Une sociologie pragmatique de l'alerte et du risque. Paris, EHESS.

DAB W., 2006 - L'homme malade de l'environnement. Paris, Eska.

Dab W., Goldberg M., Mengual E., Rollet C., 1998 - Décideurs tous risques. Du bon usage de l'épidémiologie. Paris, Frison-Roche/ENSP.

De Blay F., Lieutier-Colas F, 2004 - La chambre de l'allergique. La revue du praticien - Médecine générale, t. 18, n 637-638: 53-54.

Dourlens C., 2003 - Saturnisme infantile et action publique. Paris, L'Harmattan.

Dumoulin L., La Branche S., Robert C., Warin P. (éd.), 2005 - Le recours aux experts. Raisons et usages politiques. Grenoble, Presses Universitaires de Grenoble.

DUPONT Y., SURIRÉ C., 2005 - Médecine libérale et pathologies environnementales. Enquête exploitée pour le compte de la commission santé environnement de l'URML de Haute-Normandie par le LASAR, université de Caen, $45 \mathrm{p}$.

EILSTEIN D. et al., 2004 - Pollution atmosphérique: quel impact sanitaire? La revue du praticien - Médecine Générale, t. 18, nº 643: 289-291.

ESTADES J., REMY E., 2003 - L'expertise en pratique. Le cas de la vache folle et des rayons ionisants. Paris, L'Harmattan.

EVEILlaRD P., 2004 - L'actualité médicale dans les agences sanitaires et... ailleurs. La revue du praticien - Médecine générale, t. 18, n 641 : 215-216.

GARNIER R., 2003 - Pollution des sols par les dioxines. Le concours médical, t. 125-06: 334-335.

GARNIER R., 2004 - Incinération des déchets. Le concours médical, t. $126-37: 2180$.

Gérin M., Gosselin P., Cordier S., Viau C., Quenel P., Dewailly E. (éd.), 2003 - Environnement et santé publique. Fondements et pratiques. Québec, Edisem.

Gilbert C. (éd.), 2002 - Risques collectifs et situations de crise. Apports de la recherche en sciences humaines et sociales. Paris, L'Harmattan.

Jouzel J.-N., Landel D., 2005 - Décider en incertitude. Le cas d'une technologie à risques et de l'epidémie d'hépatite B. Paris, L'Harmattan. 
MARÉCHAUX F, 2004 - Accidents collectifs, attentats, catastrophes naturelles: conduite à tenir pour les professionnels de santé. La revue du praticien-Médecine générale, t. 18, $\mathrm{n}^{\circ}$ 646-647: 453-454.

NAHON T., 1999 - Étude des représentations de la population riveraine d'une usine d'incinération. L'exemple d'Athanor. Déchets Sciences et Techniques, $\mathrm{n}^{\circ}$ 13: 18-22.

ReIHL F.X., 2002 - Atlas de poche de Médecine de l'environnement. Éditions Maloine.

Robelet M., 1999 - Les médecins sous observation. Mobilisations autour du développement de l'évaluation médicale en France. Politix, $\mathrm{n}^{\circ} 46,71-97$.

TORNY D., 1997 - Surveiller et contenir. Maladies à prions et politique de la vigilance. Revue française des affaires sociales, 51, n 3-4: 221-231. VAillancourt J.-G., COTNOIR L., 2003 - "Sociologie de la santé environnementale ». In Gérin M. et al. (éd.) : Environnement et santé publique. Fondements et pratiques, Québec, Edisem: 251-257.

ZONABEND F, 1993 - Au pays de la peur déniée. Communications, 57 : 121-130. 
Annexe

Liste et intitulés des 21 articles traitant des liens santé/environnement (pollution de type industriel et risques technologiques divers) parus dans trois revues médicales de janvier 2003 à mars 2005

Classement thématique et par ordre chronologique

(avec indication de la taille de l'article en fin de chaque référence)

\begin{tabular}{|c|c|}
\hline $\begin{array}{l}\text { Rubriques } \\
\text { thématiques }\end{array}$ & Références \\
\hline \multirow[t]{7}{*}{$\begin{array}{l}\text { Pollution } \\
\text { de type industriel } \\
(\mathrm{n}=7)\end{array}$} & $\begin{array}{l}\text { RP-Mono, } 2003,53(17) \text {, (revue de presse), } \\
\text { Même en dessous des normes sanitaires, } \\
\text { l'exposition à l'ozone aggrave l'asthme chez l'enfant } \\
(1 / 3 \text { p.) }\end{array}$ \\
\hline & $\begin{array}{l}\text { RP-Mono, } 2004,54(16) \text {, (revue de presse), } \\
\text { La pollution est nocive pour l'appareil respiratoire } \\
\text { de l'enfant (1/3 p.) }\end{array}$ \\
\hline & $\begin{array}{l}\text { RP-MG, 2004, } 18, n^{\circ} 643 \text {, } \\
\text { Pollution atmosphérique: quel impact sanitaire? ( } 3 \text { p.) }\end{array}$ \\
\hline & $\begin{array}{l}\text { CM, 2003, 125-06, Pollution des sols par les dioxines } \\
\text { (Consultations/Environnement) (1/2 p.) }\end{array}$ \\
\hline & $\begin{array}{l}\text { CM, 2004, 126-23, } \\
\text { Déchets ménagers: limiter la toxicité (4 p.) }\end{array}$ \\
\hline & $\begin{array}{l}\text { CM, 2004, 126-37, Incinération des déchets } \\
\text { (Consultations/Environnement) (1/2 p.) }\end{array}$ \\
\hline & $\begin{array}{l}\mathrm{CM}, 2005,127-10, \text { Pollution et infarctus, } \\
\text { une liaison dangereuse ( } 2 \text { p. } 1 / 2)\end{array}$ \\
\hline \multirow[t]{7}{*}{$\begin{array}{l}\text { Risques } \\
\text { technologiques } \\
\text { divers }(n=14)\end{array}$} & $\begin{array}{l}\text { RP-Mono, 2004, } 54 \text { (15), } \\
\text { Risques sanitaires liés aux irradiations. } \\
\text { Radioprotection (7 p.) }\end{array}$ \\
\hline & $\begin{array}{l}\text { RP-Mono, } 2005,55 \text { ( } 2) \text {, (revue de presse), } \\
\text { L'exposition chronique au plomb pourrait favoriser } \\
\text { la cataracte }(1 / 3 \text { p.) }\end{array}$ \\
\hline & $\begin{array}{l}\text { RP-MG, } 2003,17, \mathrm{n}^{\circ} 604 \text {, éditorial, } \\
\text { Risque bioterroriste: les généralistes en première ligne? } \\
(1 \mathrm{p.})\end{array}$ \\
\hline & $\begin{array}{l}\text { RP-MG, 2005, T } 19, n^{\circ} 680 / 681 \text {, veille documentaire, } \\
\text { État des lieux des impacts sanitaires du bruit }\end{array}$ \\
\hline & $\begin{array}{l}\text { CM, 2003, 125-09, Ligne à moyenne tension et santé } \\
\text { (Consultations/Environnement) }(1 / 2 \mathrm{p} .)\end{array}$ \\
\hline & $\begin{array}{l}\text { CM, 2003, 125-14/15, Vaisselle peinte et alimentation } \\
\text { (Consultations/Toxicologie) ( } 2 / 3 \text { p.) }\end{array}$ \\
\hline & $\begin{array}{l}\text { CM, 2003, 125-14/15, Herbicide et culture de légumes } \\
\text { (Consultations/Toxicologie) (1/3 p.) }\end{array}$ \\
\hline
\end{tabular}




\begin{tabular}{|c|c|}
\hline $\begin{array}{l}\text { Rubriques } \\
\text { thématiques }\end{array}$ & Références \\
\hline \multirow[t]{7}{*}{$\begin{array}{l}\text { Risques } \\
\text { technologiques } \\
\text { divers }(n=14)\end{array}$} & $\begin{array}{l}\text { CM, 2003, 125-16/17, } \\
\text { Contrôle de la qualité de l'eau } \\
\text { (Consultations/Hygiène) (1/3 p.) }\end{array}$ \\
\hline & $\begin{array}{l}\text { CM, 2003, 125-30, Risques des lignes à haute tension } \\
\text { (Consultations/Environnement) (1 p. 1/4) }\end{array}$ \\
\hline & $\begin{array}{l}\text { CM, 2003, 125-30, Éoliennes et champ } \\
\text { électromagnétique (Consultations/Environnement) } \\
\text { (1/4 p.) }\end{array}$ \\
\hline & $\begin{array}{l}\text { CM, 2004, 126-22, Risques des éoliennes } \\
\text { (Consultations/Environnement) (1/2 p.) }\end{array}$ \\
\hline & $\begin{array}{l}\text { CM, 2004, 126-22, Ostéoporose et champ magnétique } \\
\text { (Consultations/Environnement) (1 p.) }\end{array}$ \\
\hline & $\begin{array}{l}\text { CM, 2004, 126-29, Intoxication oxycarbonée } \\
\text { au domicile: dépistage des risques en médecine } \\
\text { générale (5 p.) }\end{array}$ \\
\hline & $\begin{array}{l}\text { CM, 2004, 126-28, Entretien des bacs à sable } \\
\text { (Consultations/Hygiène) }(1 / 2 \text { p.) }\end{array}$ \\
\hline
\end{tabular}

RP-Mono: La revue du praticien - Monographie RP-MG : La revue du praticien - Médecine générale CM: Le concours médical 


\section{Évaluation \\ de la communication \\ en matière de risques \\ liés à l'utilisation \\ des pesticides \\ au Nord-Cameroun ${ }^{1}$}

\section{Henry TOURNEUX}

Selon une dépêche de la FAO (Food and Agriculture Organization of the United Nations) et du Pnue (Programme des Nations unies pour l'environnement) (22 septembre 2004, Rome/Genève), " on estime qu'il y aurait chaque année [dans le monde] de un à cinq millions de cas d'empoisonnements aux pesticides ${ }^{2}$, entraînant la mort de plusieurs milliers d'ouvriers agricoles. La plupart de ces empoisonnements ont lieu dans les pays en développement où les normes sanitaires sont souvent insuffisantes, voire totalement inexistantes. Quoique ces pays n'utilisent que 25 pour cent de la production mondiale de pesticides, ils comptent pour 99 pour cent des décès qui leur sont attribués».

1 Ce texte est une version remaniée de "Une évaluation de l'efficacité des pictogrammes phytosanitaires dans la protection contre les risques liés à l'utilisation des pesticides », chapitre de l'ouvrage suivant: H. Tourneux, 2006, La Communication technique en langues africaines, Paris, Karthala: 107-124.

2 L'OMS a des estimations un peu plus basses: elle estime que 3 millions de personnes sont victimes d'intoxication par pesticides chaque année et que 200000 d'entre elles meurent. Les chiffres restent cependant impressionnants. 
Toujours selon la même dépêche, "un exemple des risques particuliers que connaissent les ouvriers agricoles des pays en développement nous vient du Sénégal. Il y a quelques années, des fonctionnaires sénégalais eurent vent de mystérieux cas d'empoisonnements en zones rurales. Après enquête, il s'agissait de fièvres, de douleurs abdominales et pulmonaires, [de] vomissements, [d'] insomnies, allant parfois jusqu'à la mort. Leurs études les amenèrent à découvrir une préparation de pesticides utilisée en poudre sur les graines [...] plantées par les producteurs d'arachides".

\section{L'utilisation des pesticides au Nord-Cameroun}

Au nord du Cameroun, les tonnages de pesticides organiques de synthèse utilisés annuellement sont très importants, du fait de leur emploi massif dans la culture cotonnière. Ces produits sont majoritairement fournis aux paysans par la société cotonnière locale. Une masse indéterminée d'autres produits vient du Nigeria par le canal de la contrebande.

Les paysans producteurs de coton disposant d'une dotation de pesticides (qu'ils rembourseront sur les produits de leur récolte) détournent parfois une partie de cette dotation, soit pour l'utiliser eux-mêmes ailleurs que dans la culture cotonnière, soit pour la revendre à des maraîchers notamment. La toxicité des produits employés dans l'agriculture cotonnière exclut pourtant leur emploi en maraîchage. La plupart des légumes (brèdes, salades, tomates) que l'on trouve sur les marchés en saison sèche et chaude (période où la pression des ravageurs est maximale dans les jardins) sont littéralement imbibés de ces produits. Aucun système de contrôle n'intervient pourtant à ce niveau.

Toujours en saison sèche, certains braconniers empoisonnent avec des pesticides destinés au coton les mares où viennent s'abreuver les oiseaux (oies de Gambie, canards divers, grues couronnées, hérons, etc.). Des mammifères peuvent aussi se rendre aux mêmes points d'eau (petit bétail, antilopes, etc.). Les animaux ainsi 
empoisonnés sont plumés ou dépouillés et vidés, puis livrés au consommateur (restaurants de Kousseri, notamment, ou particuliers, qui ne savent pas comment ces animaux ont été tués).

Un autre détournement de l'usage des pesticides consiste à empoisonner des biefs de pêche. La totalité des poissons se retrouvent presque instantanément le ventre à l'air. Ces poissons sont alors recueillis et commercialisés en l'état, à moins qu'on ne les fasse sécher ou qu'on ne les fume avant de les mettre sur le marché.

Un dernier usage des pesticides du coton se retrouve lors du fumage et du séchage du poisson, principalement en saison des pluies, mais pas uniquement. Le poisson éviscéré et fendu en deux est trempé dans un bain de pesticide avant d'être étalé à sécher sur des nattes ou sur des claies, ou avant d'être mis dans (sur) le fumoir. À la saison des pluies, le nombre de ravageurs susceptibles d'attaquer le poisson sec et fumé est très important, et les pêcheurs craignent de voir leur marchandise dévalorisée par la présence de larves très nombreuses qui dévorent en quelque jour toute la chair, ne laissant que les parties osseuses et la peau.

Tout récemment, j'ai vu utiliser un autre pesticide sur du sorgho rouge destiné à l'alimentation humaine (marché de Pouss, mai 2006). Les grains étaient saupoudrés d'une poudre rose (fongicide-insecticide) destinée à traiter les semences. Ils étaient anormalement intacts alors qu'en la saison, ils auraient dû être fortement attaqués par les bruches.

\section{Les pictogrammes phytosanitaires}

Selon la FAO (1995: 16), « un pictogramme est un symbole qui véhicule un message sans l'aide de mots. L'ensemble (des pictogrammes ci-dessous) a été conçu par le Gifap (Groupement international des associations nationales des fabricants de produits chimiques) en collaboration avec la FAO, dans le but de communiquer l'information essentielle relative à la sécurité (key safety information) aux utilisateurs de différents pays et de 
niveaux d'instruction variés». Lorsque l'on connaît la difficulté qu'il y a à diffuser des messages adéquats et compréhensibles dans toutes les langues parlées par les utilisateurs de pesticides dans le monde, et en Afrique en particulier, on mesure l'importance qu'aurait une méthode simple et universelle de communiquer.

Dans la version précédente de son document (la seule dont je disposais en 1993), la FAO déclarait que ses pictogrammes avaient été sélectionnés "sur la base d'une enquête menée auprès de 1000 agriculteurs et ouvriers agricoles de 42 pays ». Les auteurs du document poursuivaient en affirmant "que des personnes de cultures et de degrés d'instruction extrêmement différents comprenaient fort bien les messages que ces pictogrammes étaient destinés à leur transmettre». C'est cette dernière affirmation, trop optimiste à mon sens, qui m'a poussé à réaliser une enquête auprès d'un échantillon aléatoire de paysans du Nord-Cameroun.

Ci-dessous sont donnés d'abord les pictogrammes actuellement recommandés, et après, les pictogrammes qui avaient cours à l'époque de l'enquête. On constate qu'il y en a 14 contre 13 précédemment. Le pictogramme à tête de mort a été retiré; les lunettes ont été remplacées par une visière transparente, et deux ont été ajoutés représentant des vêtements de protection.

\section{Nouvelle version (1995) des pictogrammes de la FAO}

Dans cette nouvelle version, on se contente de regrouper les pictogrammes en fonction de leur domaine de référence: stockage des pesticides ( 1 pictogramme), manipulation des pesticides lors du dosage et de la pulvérisation ( 3 pictogrammes), conseils donnés aux personnes qui les épandent dans les champs (8 pictogrammes), mises en gardes relatives à l'environnement ( 2 pictogrammes). Les intitulés ci-dessous sont ceux qui se trouvent dans le document original.

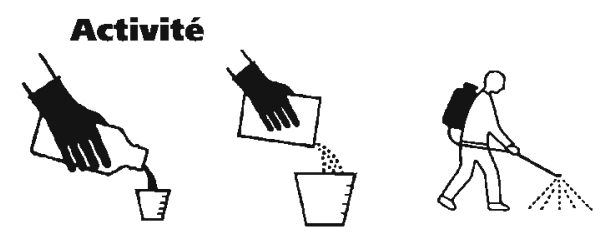




\section{Stockage}

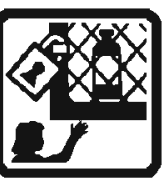

Conseil
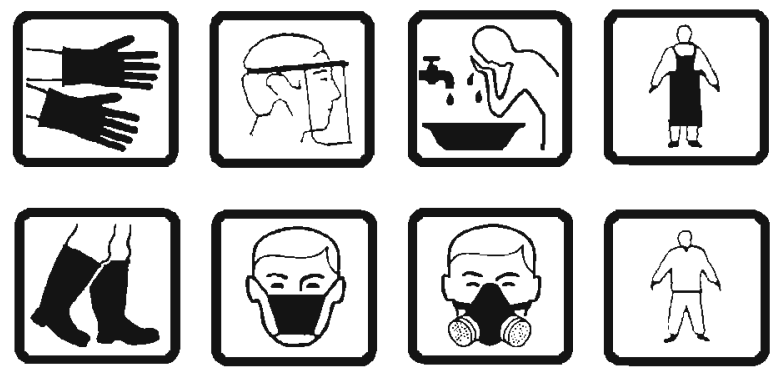

Mise en garde

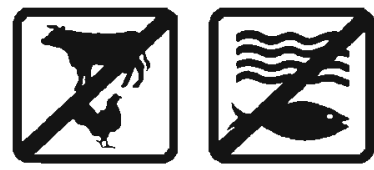

\section{Pictogrammes en circulation lors de l'enquête (1993)}

La première série de pictogrammes destinés à l'étiquetage des produits phytosanitaires était accompagnée d'une explication verbale en français, consistant en une phrase complète (numéros $1,2,3$ ), en une consigne en style télégraphique (verbe à l'infinitif, sans sujet, numéros 4, 10 et 13) ou encore en un simple groupe nominal (numéros 11,12 ).

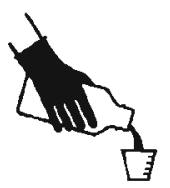

1. Voici comment il faut manipuler le liquide concentré

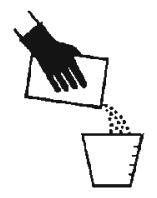

2. Voici comment il faut manipuler le concentré sec 


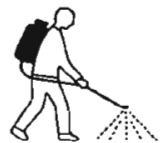

3. Voici comment il faut appliquer le produit

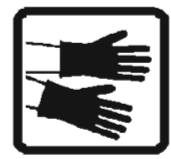

4. Porter des gants

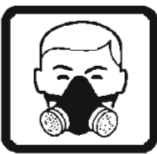

5. Porter un respirateur

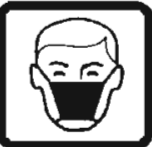

6. Porter un masque de protection couvrant le nez et la bouche

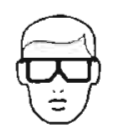

7. Porter des lunettes de protection

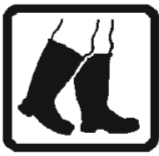

8. Porter des bottes

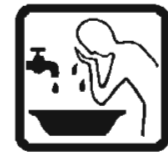

9. Se laver après l'emploi

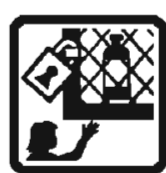

10 Garder sous clé hors de la portée des enfants

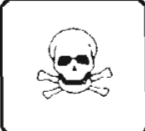

11. Danger

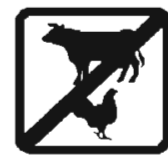

12. Dangereux, nocif pour les animaux

13. Dangereux, nocif pour les poissons; éviter de contaminer les lacs, les rivières, les étangs ou les cours d'eau 


\section{L'enquête}

J'ai donc fait réaliser une enquête ${ }^{3}$ auprès d'un échantillon aléatoire de 203 personnes, dont 8 femmes, résidant dans un rayon de $30 \mathrm{~km}$ autour de Maroua (chef-lieu de la province de l'Extrême-Nord du Cameroun), et appartenant à une vingtaine d'ethnies différentes, parmi lesquelles on relevait 42 Peuls et assimilés, 26 Mofou de Mokong, 23 Guiziga, 15 Toupouri, 11 Zoumaya, 9 Kanouri, 9 Mandara, 8 Moundan, 8 Massa, 8 Mafa, 8 Moussey, etc.; $76 \%$ de ces personnes étaient analphabètes, les autres ayant généralement passé un ou deux ans à l'école primaire.

Chaque enquêté était reçu isolément et l'entretien avait lieu en fulfulde, langue véhiculaire de la région. Les enquêtés étaient tous engagés dans des activités agricoles, notamment dans la culture du coton. Après avoir noté les renseignements d'usage sur la personne, l'enquêteur lui présentait une bouteille vide d'un litre. Cette bouteille avait contenu un pesticide et portait l'étiquette du fabricant (nom commercial du produit, nom du fabricant, matière active, etc.) ainsi qu'un bandeau rouge sur lequel figuraient, au centre, une tête de mort et, de part et d'autre, une séquence de pictogrammes indiquant, à gauche, les précautions à prendre lors de la manipulation et de la préparation du produit, et à droite, les précautions à prendre lors de la pulvérisation.

Ensuite, l'enquêteur présentait un par un, à une échelle de $4 \mathrm{~cm} \times 4 \mathrm{~cm}$, les pictogrammes de la FAO collés sur une petite fiche plastifiée, puis il posait la question suivante: "Si vous trouvez les dessins suivants sur une bouteille d'insecticide destiné à traiter le coton, par exemple, qu'est-ce que cela signifie? ». Je n'ai pas tenté de faire interpréter une séquence complète de pictogrammes; cela aurait posé le problème supplémentaire de l'orientation de la lecture (de droite à gauche et (ou) de gauche à droite), problème qui se poserait même avec des personnes alphabétisées, notamment si elles avaient fréquenté uniquement l'école coranique où l'on lit de droite à gauche ou de haut en bas. 


\section{Tableau des résultats}

Le tableau 1 présente de façon synthétique, pour chacun des pictogrammes, les pourcentages de réponses obtenues au cours de l'enquête en milieu paysan, en fonction de leur pertinence ${ }^{4}$. Leur classement s'organise ainsi:

- incompris: le pictogramme ne suscite aucune interprétation;

- juste: le pictogramme est bien interprété;

- partiel: le message n'est pas compris intégralement;

- faux: le message est mal interprété.

Tableau 1.

Pourcentages de réponses selon leur pertinence

\begin{tabular}{|ccccc|}
\hline Pictogramme & Incompris & Juste & Partiel & Faux \\
\hline $\mathbf{1}$ & 17 & 4,5 & 71 & 7,5 \\
\hline $\mathbf{2}$ & 14 & 0,5 & 55 & 30,5 \\
\hline $\mathbf{3}$ & 1,5 & 64 & 4 & 30,5 \\
\hline $\mathbf{4}$ & 6 & 89,5 & 0 & 4,5 \\
\hline $\mathbf{5}$ & $\mathbf{2 8}$ & 0 & 55 & 17 \\
\hline $\mathbf{6}$ & 24 & 28 & 40,5 & 7,5 \\
\hline $\mathbf{7}$ & 3 & 83 & 14 & 0 \\
\hline $\mathbf{8}$ & 2 & 81 & 15,5 & 1,5 \\
\hline $\mathbf{9}$ & 20,5 & 58,5 & 5 & 16 \\
\hline $\mathbf{1 0}$ & 54,5 & 39 & 0 & 6,5 \\
\hline $\mathbf{1 1}$ & 43 & 39,5 & 0 & 17,5 \\
\hline $\mathbf{1 2}$ & 17,5 & 58 & 0 & 24,5 \\
\hline $\mathbf{1 3}$ & 26 & 11 & 46,5 & 16,5 \\
\hline
\end{tabular}

Voici les réponses détaillées obtenues auprès des paysans pour quelques-uns de ces pictogrammes:

\section{Interprétations du pictogramme 9}

Incompris

- Ne comprend pas $(20,5 \%)$

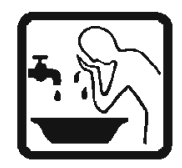

4 En grisé, les résultats correspondant aux pictogrammes retires de la circulation par la FAO. 
Juste

- Après avoir traité les cotonniers, on doit se laver la figure avec l'eau du robinet ( $42 \%$ )

- Il faut se laver des pieds à la tête après le traitement (9\%)

- Il faut se laver les mains et la figure après le traitement (7,5\%)

\section{Partiel}

- Après avoir touché le produit, il faut se laver les mains (3\%)

- Après avoir utilisé le pesticide, on doit se laver les mains et se les mettre sous le nez pour voir si elles sentent encore le produit $(2 \%)$

Faux

- Il ne faut pas boire d'eau dans des mains souillées par l'insecticide $(4,5 \%)$

- Il faut mélanger le pesticide avec de l'eau (4\%)

- Après le traitement, on doit boire l'eau du robinet (2\%)

- On désinfecte l'eau sale avec le produit (1,5\%)

- Il ne faut pas boire le produit $(1,5 \%)$

- Il faut sentir le produit avant de traiter pour voir s'il a une bonne odeur $(1,5 \%)$

- On doit fermer les yeux avant de toucher le produit traitant ( $1 \%)$

\section{Interprétations du pictogramme 10}

Incompris

- Ne comprend pas $(54,5 \%)$

Juste

- Ne pas laisser le produit à la portée des enfants (ou des femmes) $(24,5 \%)$

- Il faut garder le produit hors d'atteinte (10,5\%)

- Il faut garder le pesticide dans une armoire fermée avec un cadenas (3\%)

- Il faut garder la bouteille de produit dans un magasin avant et après le traitement ( $1 \%$ ) 
Faux

- Il faut garder le produit au bord d'une fenêtre (2\%)

- Il faut suspendre au mur la bouteille d'insecticide (1,5\%)

- Il ne faut pas garder le produit au bord d'une fenêtre (1\%)

- Il faut garder le produit dans un endroit élevé (1,5\%)

- On mélange le produit avec de l'eau (0,5\%)

\section{Interprétations du pictogramme 12}

\section{Incompris}

- Ne comprend pas $(17,5 \%)$

Juste

- Le produit tue les vaches et les poules (48\%)

- Il ne faut pas verser le produit dans un endroit où boivent des vaches et des poules $(5,5 \%)$

- Il ne faut pas verser ce produit dans un endroit où paissent les bcufs (2\%)

- Le produit rend les poules et les vaches malades (1,5\%)

- On éloigne les vaches et les poules avant de répandre le produit sur le champ (1\%)

Faux

- On utilise le produit pour engraisser les vaches et les poules (9\%)

- Le produit est un vaccin contre les maladies des vaches et des poules $(6 \%)$

- Il ne faut pas élever la vache et la poule dans la même case (3\%)

- Le produit tue les parasites des vaches et des poules ( $2 \%$ )

- Si on répand le produit sur les plantations, on obtiendra de bonnes récoltes, des vaches et des poules $(1,5 \%)$

- Il ne faut pas abandonner une vache ou une poule sur la route (1\%)

- Le produit soigne les vaches et les poules (1\%)

- Ne pas laisser le produit à la portée des vaches et des poules $(0,5 \%)$

- Le produit soûle les vaches et les poules $(0,5 \%)$ 


\section{Interprétations du pictogramme 13}

Incompris

- Ne comprend pas (26\%)

Juste

- On ne doit pas verser le produit dans une mare qui contient des poissons $(11 \%)$

Partiel

- Le produit tue les poissons dans l'eau (45\%)

- Le produit pollue l'eau des rivières $(1,5 \%)$

Faux

- On nourrit les poissons avec ce produit (4\%)

- Le produit sert à pêcher (4\%)

- Le produit favorise la reproduction des poissons (3\%)

- Le produit soûle les poissons (1,5\%)

- Il ne faut pas manger de poissons contaminés par le produit ( $1 \%$ )

- On utilise le produit pour engraisser les poissons ( $1 \%$ )

- On verse le produit dans l'eau pour soigner les poissons ( $1 \%$ )

- Avant de cuire les poissons frais, il faut les laver avec le produit $(0,5 \%)$

- On doit toujours garder les poissons dans une caisse avant de répandre le produit $(0,5 \%)$

\section{Commentaires}

Dans les résultats obtenus, on constate que tel pictogramme peut avoir un bon score de réponses justes ( $n^{\circ} 9,12,13$ par exemple), mais avoir des réponses fausses tellement dangereuses qu'il faut probablement l'éliminer:

- on désinfecte l'eau sale avec le produit $\left(n^{\circ} 9\right)$

-on utilise le produit pour engraisser les vaches, les poules, les poissons $\left(n^{\circ} 12-13\right)$

-le produit tue les parasites des vaches et des poules $\left(n^{\circ} 12\right)$

-le produit est un vaccin contre les maladies des vaches et des poules $\left(\mathbf{n}^{\circ} 12\right)$

-le produit sert à pêcher $\left(n^{\circ} 12\right)$, etc. 
La réponse juste peut être suivie d'une clause supplémentaire qui fera qu'on ne suivra pas la consigne; par exemple:

- après avoir traité les cotonniers, on doit se laver la figure avec l'eau du robinet $\left(n^{\circ} 9\right)$.

Comme généralement on ne dispose pas de l'eau du robinet, mais de l'eau du puits, on ne se lavera pas après le traitement, l'élément important retenu par le paysan étant "eau du robinet».

La réponse peut susciter une fausse interprétation, c'est-àdire non voulue par l'auteur du pictogramme, mais juste dans l'absolu :

-il ne faut pas verser le produit dans un verre que des personnes utilisent $\left(n^{\circ} 1\right)$

-le produit sent mauvais $\left(n^{\circ} 6\right)$

- il ne faut pas boire le pesticide $\left(n^{\circ} 6\right)$, etc.

La réponse fausse peut être anodine:

- il ne faut pas élever la vache et la poule dans la même case $\left(n^{\circ} 12\right)$

- il ne faut pas abandonner une vache ou une poule sur la route $\left(n^{\circ} 12\right)$

La réponse fausse peut induire un comportement dangereux:

- on désinfecte l'eau sale avec le produit ( $\left.{ }^{\circ} 9\right)$

- il faut sentir le produit avant de traiter pour voir s'il a une bonne odeur $\left(n^{\circ} 9\right)$

- il faut garder le produit au bord d'une fenêtre $\left(n^{\circ} 10\right)$

- on utilise le produit pour engraisser les vaches, les poules, les poissons $\left(n^{\circ} 12-13\right)$

- le produit tue les parasites ou est un vaccin des vaches et des poules $\left(n^{\circ} 12\right)$

- le produit sert à pêcher $\left(n^{\circ} 13\right)$

- avant de cuire les poissons frais, il faut les laver avec le produit $\left(n^{\circ} 13\right)$, etc. 


\section{Conclusions}

Il faut bien constater que le pictogramme n'est pas en soi un moyen suffisant pour communiquer une information par-delà des barrières linguistiques et culturelles. D'abord, sa taille sur l'étiquette du produit est généralement minuscule $(1,5 \mathrm{~cm} \times 1,5 \mathrm{~cm}$ selon les recommandations de la $\mathrm{FAO}$, mais souvent moins dans la réalité). Ensuite, il y a des pesticides qui sont vendus au détail, donc hors emballage (c'est le cas à Maroua du produit destiné à traiter les semences; on le manipule à la main, sans masque, pour le conditionner dans des lambeaux de film plastique). À BoboDioulasso, on trouve également des produits "anti-termites", notamment, conditionnés manuellement dans de petits sachets.

Par ailleurs, suivant la langue que l'on utilise, le pesticide est présenté sous un jour différent; en jula, par exemple (Burkina, Côte d'Ivoire notamment), le pesticide est appelé «poison» [bàga, pósoni], ce qui met immédiatement l'utilisateur en alerte. En fulfulde, le pesticide comme le médicament ou le remède s'appellent [lekki]; le nom présente la même ambivalence que le grec [phármakon]. Par ailleurs, dans la mentalité locale, on ne peut considérer comme un poison ce qui ne tue pas sur place. Le pesticide employé comme ichtyotoxique ne tue pas [mbaran] le poisson, mais le soûle [wuykinan], formulation qui contribue également à la banalisation de l'usage de la substance toxique.

Aucune information n'est donnée sur la rémanence des organophosphorés, organochlorés et carbamates dans l'organisme, par exemple. On sait que l'exposition répétée, même à de faibles doses de pesticides, et surtout la bio-accumulation ${ }^{5}$ peuvent avoir des conséquences sur la croissance, le développement et la reproduction (PHILOGĖNE, 2005: 180). Si l'on ne meurt pas en respirant de la poudre insecticide, c'est la preuve que le produit n'est pas si

5 «Processus d'accumulation d'une substance dans tout ou partie d'un organisme vivant, via la chaîne trophique ou un écosystème [...]. II s'agit de l'accumulation des produits toxiques dans les tissus et organes des organismes. Cette situation est le résultat d'une absorption sélective, de la non-dégradation de la molécule, et de la capacité des cellules à entreposer cette dernière, à cause, par exemple, de sa lipophilie» (PHILogène, 2005: 173). 
dangereux qu'on le dit, pense-t-on. Cependant, dans une localité comme Maga (Cameroun), située près du lac de barrage qui sert à irriguer la rizière, certaines personnes commencent à se poser des questions sur la nocivité de l'emploi des pesticides sur le poisson sec et fumé.

Le pictogramme reste un outil utile pour la prévention des risques liés à l'utilisation des pesticides, mais les fabricants de ces produits ne doivent pas se réfugier derrière cette protection pour se décharger de toute responsabilité dans les accidents fréquents qui surviennent. De même qu'ils n'hésitent pas à consacrer des sommes importantes à la promotion de leurs produits, ils devraient impérativement en consacrer autant à l'information et à la formation des utilisateurs. Dans ce contexte, le pictogramme servirait de simple aide-mémoire. En aucun cas, il ne devrait être considéré comme un moyen d'information suffisant.

\section{Références bibliographiques}

FAO, 1995 - Guidelines on Good Labelling Practice for Pesticides. Rome, $51 \mathrm{p}$.

Multigner L., Cordier S., JÉGOU B, 2005 - «Effets adverses des produits phytosanitaires sur la santé humaine». In Regnault-Roger $C$. et al. (coord.) : 243-259.

PHILOGĖNe B. J.-R., 2005 - «Effets non intentionnels des pesticides organiques de synthèse: impact sur les écosystèmes et la faune». In Regnault-Roger C. (coord.), 2005: 171-187.

REgnaUlt-Roger C. (coord.), avec la coll. de FABRes G., PHILOGĖNe B. J.-R., 2005 - Enjeux phytosanitaires pour l'agriculture et l'environnement, Londres - Paris - New York, TEC \& DOC, LVII + 1013 p.

TOURNEUX H., 1993 a - La perception des pictogrammes phytosanitaires par les paysans du Nord-Cameroun. Coton et Fibres Tropicales, 48, 1 : $41-48$.

TOURNEUX H., 1993b - Smallholder understanding of phytosanitary pictograms in North Cameroon. Coton et Fibres Tropicales, 48, 1, 49-56 (version anglaise de l'article ci-dessus). 
TOURNEUX H., 1994a - «Quelques réflexions sur la perception des pictogrammes phytosanitaires par les paysans du Cameroun septentrional ». In: Réunion phytosanitaire de coordination, cultures annuelles, Afrique centrale, 26-29 janvier 1994, Maroua (Cameroun), Cirad-IRA, s.l. : 222228.

TOURNEUX H., 1994b - La interpretación campesina de los pictogramas fitosanitarios. Agriculture et Développement, número especial, Diciembre: 21-23.

TOURNEUX H., 1994c - Farmer's interpretation of pesticide pictograms. Agriculture et Développement, special issue, December : 21-23.

TOURNEUX H., 2006 - La communication technique en langues africaines : l'exemple de la lutte contre les ravageurs du cotonnier (Burkina Faso/Cameroun). Paris, Karthala, 158 p. 



\section{Pratiques sociales}

et risque mercuriel

des populations

riveraines du Río Beni

en Amazonie bolivienne

\section{Céline TSCHIRHART \\ Pascal HANDSCHUMACHER \\ Dominique LAFFLY}

Les processus du mercure $(\mathrm{Hg})$ et ses conséquences sur la santé sont connus, notamment en milieu amazonien. Le MeHg ( $\mathrm{Hg}$ organique, forme la plus toxique du $\mathrm{Hg}$ ) est un neurotoxique puissant et joue un rôle dans l'existence de troubles neurologiques (CARmouze et al., 2001). La transformation du $\mathrm{Hg}$ en $\mathrm{MeHg}$ s'effectue grâce à l'action de bactéries sulfatoréductrices existant notamment sur la végétation aquatique (JENSEN, JORNELOV, 1969). La contamination des populations ne se fait pas par l'ingestion d'eau transportant du MeHg mais par consommation de poisson lui-même contaminé. En effet, le MeHg est biomagnifiable et bioaccumulable, apparaissant ainsi en concentrations croissantes tout au long de la chaîne trophique (CARMOUZE $e t$ al., 2001). Les poissons carnivores et piscivores situés en bout de chaîne concentrent donc les teneurs maximales de MeHg. Une consommation régulière, même modérée, de ces poissons présente ainsi un risque pour la santé humaine. En effet, l'impact en termes de morbidité est d'autant plus important que le degré de contamination est fort, celui-ci augmentant par accumulation. D'après l'OMS (WHO, 1990), 
le seuil à partir duquel il est possible de constater l'apparition des symptômes est compris entre 50 et $125 \mu \mathrm{g} / \mathrm{g}$ de concentration de mercure dans les cheveux. Pour les femmes enceintes, il est considéré qu'une concentration supérieure à $10 \mu \mathrm{g} / \mathrm{g}$ dans leurs cheveux peut accroître les risques pour le bon développement neuronal et psychomoteur de l'enfant, par passage de la barrière placentaire lors de la grossesse. Les femmes enceintes et les enfants constituent ainsi la population la plus vulnérable. Mais ces seuils sont à considérer comme des valeurs indicatives: des anomalies neurologiques ont été observées pour des concentrations inférieures à $50 \mu \mathrm{g} / \mathrm{g}$ de mercure dans les cheveux chez des populations riveraines du Tapajós (Brésil) ayant une alimentation très riche en poissons (LEBEL et al., 1998), alors que des individus rencontrés au Brésil et présentant une concentration de $100 \mu \mathrm{g} / \mathrm{g}$ dans les cheveux, ne montraient pas de symptômes (Dorea, comm. pers.). On admet pourtant actuellement que le seuil d'innocuité pour les personnes les plus sensibles (enfants et femmes enceintes) se situe autour de $5 \mu \mathrm{g} / \mathrm{g}$ de $\mathrm{Hg}$ dans les cheveux (MURESAN PASLARU, 2006).

Dans le cas de la Bolivie, l'activité d'orpaillage s'est particulièrement intensifiée depuis une cinquantaine d'années dans le bassin versant du Río Beni (MAURICE-BOURGoin et al., 1999), or le Hg est utilisé par les orpailleurs pour amalgamer l'or et les résidus sont la plupart du temps rejetés dans le milieu. Mais le mercure existe également à l'état naturel dans les sols, en particulier dans les sols ferrallitiques des forêts équatoriales. Sa libération s'opère par des phénomènes d'érosion et de ruissellement (naturels et anthropiques), contribuant ainsi à la contamination des cours d'eau depuis les contreforts andins jusque dans les plaines d'inondation amazoniennes (CARMOUZE et al, 2001). Le Hg ne se transforme en MeHg que dans des conditions bien particulières: milieux anaérobies, ou très peu oxygénés, connaissant une "intense activité microbiologique due à la dégradation des matières organiques inondées ou produites in situ " (CARMOUZE et al., 2001), conditions réunies qu'à partir de l'entrée dans la plaine d'inondation du Río Beni. En aval de Rurrenabaque, point d'entrée dans la plaine d'inondation, existe une zone de subsidence (Dumont et al., 1991) qui favorise le dépôt de la quantité de sédiments chargé en $\mathrm{Hg}$ transportée depuis le haut bassin (MAURICE-BOURGOIN, 2001). 


\section{Point d'entrée dans la plaine à Rurrenabaque}

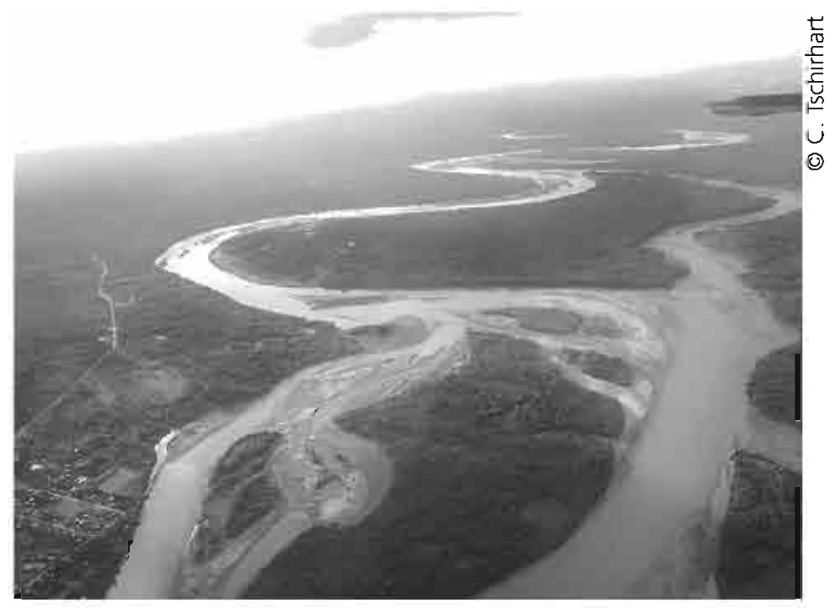

Le développement d'une végétation aquatique, notamment dans les lagunas (lacs de taille variable créés par le recoupement des méandres lors des divagations du fleuve dans son lit majeur), favorise les processus de méthylation $\mathrm{du} \mathrm{Hg}$. On peut donc distinguer un cours supérieur caractérisé par une contamination du milieu en raison des phénomènes d'érosion et de transport de matériaux contaminés par le Hg et un cours inférieur dans lequel la transformation en MeHg peut s'opérer, exposant ainsi les populations riveraines à un risque plus ou moins élevé selon leurs pratiques alimentaires.

La présence du mercure dans le Río Beni a été confirmée par des relevés effectués sur les sédiments charriés (CARMOUzE et al., 2001). De plus une enquête menée sur 16 espèces de poissons dans les eaux du Río Beni à proximité de Rurrenabaque (MAurice-Bourgoin et al., 2000), toutes consommées par les populations locales, dévoile que les espèces piscivores sont contaminées. Le risque de contamination des populations est donc réel dans cette zone d'étude, le poisson constituant une importante ressource alimentaire et devenant alors le support de la contamination humaine.

Dans ce contexte, l'ensemble des populations riveraines des cours d'eau est considéré par les services de santé comme exposé de façon homogène à une contamination chronique au $\mathrm{Hg}$, ce risque 
diminuant avec l'éloignement aux rivières. Mais on ne saurait se contenter d'un tel schéma déterministe, puisque la concrétisation du risque s'exprimera en fonction de la réalité de la consommation de poisson. Or sur ces questions de contamination par le $\mathrm{Hg}$ aucune approche n'a encore été réalisée en tenant compte de la spécificité des lieux et des sociétés. La simple proximité à la ressource "poisson " n'entraîne pas nécessairement une contamination importante, ce qui marque bien la distinction entre l'exposition théorique (la proximité au cours d'eau contaminé), l'exposition réelle (la consommation effective de la ressource contaminante) et la contamination (importance du MeHg dans l'organisme des individus). Cette relation est modulée par le jeu existant entre la proximité au fleuve, les contrôles territoriaux et leurs extensions, l'existence de droits de passage et d'usage par rapport aux communautés voisines, la disponibilité de ressources alimentaires complémentaires permettant de moduler la part prise par le poisson dans les rations alimentaires, les moyens techniques maîtrisés, les habitudes alimentaires, etc. Enfin, les liens qui existent entre les lieux, tant en termes de mobilité spatiale des individus que des filières de commercialisation des produits, sont susceptibles de jouer un rôle modulant l'expression locale des phénomènes d'exposition. La mise en évidence de l'échelle fonctionnelle de la contamination (HANDSCHUMACHER, HERVOUËT, 2004), niveau spatial permettant d'identifier la réalité des processus modulant l'expression de la contamination, devient ainsi l'enjeu majeur d'une approche géographique qui s'inscrit dans une optique d'identification des mécanismes de la variabilité du risque, pour la prévention et la sensibilisation des populations exposées.

Risque sanitaire lié à une exposition s'inscrivant dans la durée, le risque mercuriel est ainsi un indicateur des relations entretenues entre des sociétés et leurs milieux. Ce constat avait pour la première fois été effectué par J.-P. HERVOUËT (1990) à propos de l'onchocercose, maladie cumulative transmise par un vecteur, une mouche (Simulium damnosum) inféodée aux cours d'eau présentant des sites bien oxygénés nécessaires au développement de ses larves. En réfutant le schéma déterministe initialement admis liant l'importance de la maladie chez l'homme à sa proximité aux gîtes, il a mis en évidence des facteurs sociaux et comportementaux 
explicatifs de l'importance de la contamination (densités de population, pratiques de culture, structuration de l'habitat...) et montrait que l'importance de la maladie dans chaque communauté rendait compte des relations que celle-ci entretenait avec son milieu. Il fournissait ainsi des éléments à des stratégies de prévention et de lutte contre la maladie.

Sur ce "modèle" a priori sans relation (maladie neurotoxique versus maladie parasitaire à transmission vectorielle), la caractéristique fondamentale constituée par l'accumulation comme génératrice de l'importance de la morbidité permet de développer un raisonnement similaire faisant des niveaux d'endémicité les révélateurs de la construction des espaces, de leur gestion et de leurs pratiques par les sociétés.

\section{Objectifs}

Cette étude fait intervenir deux disciplines, l'épidémiologie et la géographie, mais avec un objectif fédérateur: identifier l'ensemble des facteurs responsables de la contamination des hommes par le mercure, afin de cibler les espaces et populations à risques et agir plus efficacement de manière préventive. 11 s'agit de considérer le problème de la contamination par le mercure comme un système où interagissent différents facteurs liés à la fois au milieu et aux sociétés.

Pour cette étape de l'etude, l'objectif spécifique de la partie épidémiologique consiste à caractériser l'importance de la contamination et ses déterminants. En s'appuyant sur les résultats biologiques obtenus par les médecins et permettant d'identifier la contamination de chaque individu enquêté, l'objectif initial des géographes se révèle très proche de celui des épidémiologistes: identifier les déterminants de la contamination. Mais cette première étape n'est qu'un passage obligé vers la recherche d'indicateurs de risques identifiables tant d'un point de vue spatial que social et rendant compte des inégalités face à la contamination par le $\mathrm{MeHg}$ dans les communautés riveraines du Río Beni. Il s'agit ainsi d'identifier les faits de santé et leurs articulations (territoires 
contrôlés, parcourus, gérés par les différentes communautés en fonction de leur histoire, de leurs pratiques et de leurs techniques, d'analyser l'accès aux ressources alimentaires, leur disponibilité et leur mode de gestion mais également l'accès différentiel aux structures de soin, à l'éducation, aux différentes ressources économiques) pour comprendre les processus qui ont construit des espaces présentant des caractéristiques épidémiogènes spécifiques. Ainsi, par la mise en relation des résultats de mercure dans les cheveux avec les données géographiques et la présence ou non d'une relation entre espaces, modes de vie et contamination, il sera possible de mettre en évidence les combinaisons de facteurs qui en un lieu donné exposent de manière différentielle des populations à certains risques sanitaires (SALEM, 1998) et les processus constitutifs de la création des espaces à risques.

\section{Méthode}

Les enquêtes ont été effectuées dans 15 communautés villageoises accessibles au maximum en une journée de bateau. Ces communautés sont de petits regroupements de familles administrées par un responsable (corregidor), représentant légal de la communauté devant la préfecture. En raison des contraintes de terrain, 9 communautés sur les 15 ne peuvent être atteintes que par voie fluviale. Ainsi à partir de Rurrenabaque une journée de pirogue permet de couvrir une distance d'environ $100 \mathrm{~km}$ autorisant l'accès à une diversité de situations nécessaire à l'approche comparative entre le point d'entrée dans la plaine et la dernière communauté en aval, avant une importante interruption du peuplement à partir du village de Villa Fátima. Cette unité de temps et de distance est donc retenue pour fixer les bornes géographiques de l'étude. Ces bornes, initialement décidées par les médecins, couvrent une dimension linéaire du phénomène, calquée sur le cours du Río Beni.

L'enquête épidémiologique s'est déroulée de mars 2004 à juillet 2004, en saison sèche pour des raisons d'accessibilité. Dans une première partie, un questionnaire adressé aux femmes et enfants de ces 15 communautés (les individus les plus vulnérables face à la 
Figure 1.

Situation de la zone étudiée

et répartition spatiale des types de communautés.

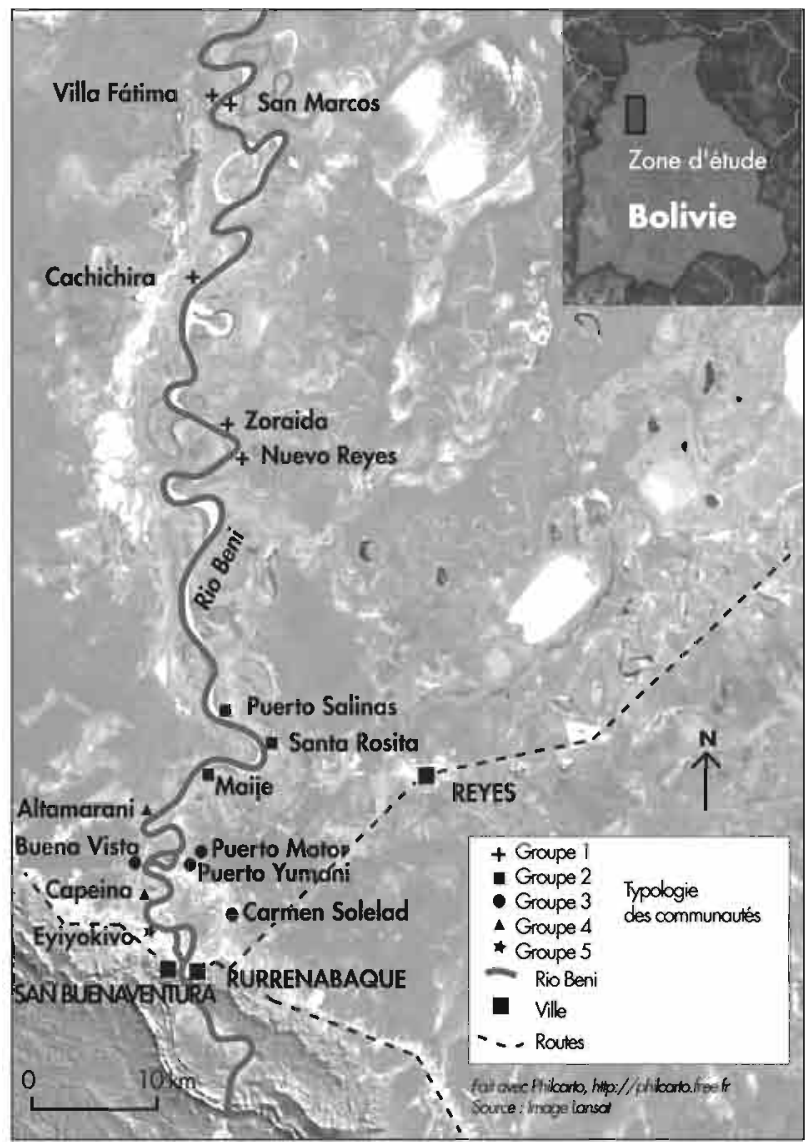

contamination) s'attache à déterminer la consommation de poisson et d'autres aliments. Un examen neurologique permet ensuite de se rendre compte des capacités neuro-psychomotrices des enquêtés, afin de les mettre en relation avec le taux de contamination. Puis une partie ciblée sur les femmes prend en considération leur profil sanitaire et les pratiques à risque susceptibles d'induire un effet de confusion avec la contamination par le MeHg: hospitalisations, consommation de cigarettes et d'alcool, passé obstétrical, examen clinique général, mesures anthropométriques, hémogramme. Des cheveux sont prélevés sur les mères et leurs enfants afin d'estimer 
leur contamination par le mercure. La teneur totale en $\mathrm{Hg}$ dans les cheveux a été analysée à partir de ces mèches collectées, par mesure en absorption atomique utilisant des vapeurs froides, au laboratoire de Qualité environnementale de l'Institut d'écologie (Cota Cota, La Paz, Bolivie), sous la responsabilité de Marc Roulet et Jaime Chincherro, après lavage, pesage et dissolution par acide fort. Un questionnaire particulier pour les enfants détermine leur histoire clinique, leur alimentation s'ils ont moins de 24 mois et leur développement psychomoteur. Puis un examen clinique est effectué, suivi de mesures anthropométriques qui serviront d'indicateur synthétique de santé. S'ensuit enfin un examen parasitologique. Au total, 565 individus ont pu être enquêtés, dont 192 femmes, dans l'ensemble des communautés.

En géographie, la méthodologie adoptée pour approcher la problématique de la contamination par le $\mathrm{MeHg}$ repose sur un délicat jeu d'échelles et de niveaux, qui se succèdent, s'articulent, s'emboîtent dans le but de révéler l'échelle fonctionnelle de la contamination. À l'échelle intervillageoise, parallèlement à l'enquête épidémiologique, une enquête géographique transversale a été menée par investigations domiciliaires sous forme de questionnaire auprès des 15 communautés sélectionnées pour l'étude. Elle avait pour objectif de dégager une typologie des relations sociétés/milieux par rapport à l'exposition et la contamination des populations par le mercure. Les enquêtes ont été menées au niveau de l'unité de production/consommation, ici au niveau de la famille, car c'est à ce niveau que se gèrent les ressources et c'est donc également à ce niveau que va se jouer en grande partie le contenu de l'assiette. Les membres de la famille ayant une connaissance globale et approfondie des pratiques du foyer ont été interrogés: chefs de famille, épouses, grands-parents... L'information a été recueillie par écrit par les enquêteurs, les enquêtés répondant oralement aux questions. Selon l'information recherchée, les questions étaient fermées ou semi-ouvertes. Le questionnaire est divisé en quatre parties et dure environ 45 minutes. La première partie vise à recenser tous les membres du foyer, afin d'obtenir un profil socio-démographique de la famille. Elle vise également à cerner la mobilité des populations en retraçant les parcours de vie. La deuxième partie du questionnaire porte sur les ressources ellesmêmes: productions agricoles, élevage, chasse, cueillette, pêche 
et exploitation de bois. Dans la troisième partie, il s'agit de comprendre les modes de recours aux structures de soin lors d'un épisode de maladie d'un des enfants au cours du dernier mois. Enfin la dernière partie recueille des informations sur le mode d'habitation et les biens matériels possédés qui pourraient constituer des indicateurs du niveau socio-économique. Au total 195 familles ont été enquêtées. L'information recueillie par famille a ensuite été agrégée dans le but de déterminer un profil de chaque communauté et de construire une typologie des communautés.

Si chacune des deux disciplines en jeu possède ses objectifs et méthodologie propre, lors de la phase de traitement des données, les deux bases d'informations doivent pouvoir être fusionnées. La mise en rapport des deux jeux de données est une étape essentielle dans cette approche globalisante interdisciplinaire. Ainsi, c'est dès la phase de collecte des données que la jointure des tables doit être pensée. En effet, comme nous l'avons vu, l'équipe d'épidémiologie adopte une approche individuelle, s'intéressant à la femme et à ses enfants. Or, l'équipe de géographie s'intéresse à la gestion des ressources au niveau familial et communautaire. Ainsi les épidémiologistes ont créé un code par individu tandis que les géographes ont créé d'abord un code unique familial. Pourtant les individus doivent pouvoir être replacés dans leur contexte familial. Ainsi, lors de l'enquête géographique, un recensement de la famille a été effectué, contenant des informations sur chaque membre de la famille: nom, prénom, sexe, âge, et statut familial puis la(es) langue(s) parlée(s), niveau d'études, lieu de naissance, lieux de vie, nombre d'années successives passées dans la communauté et profession. Ce recensement n'a pas pour seul but de s'accorder aux données médicales, car l'un des points centraux de la méthode suivie en géographie pour cette problématique est l'articulation de différentes échelles. En effectuant ce recensement, la mise en perspective de données individuelles et collectives devient possible.

L'enquête géographique a permis de recueillir un nombre très important de variables (265), mais sur l'ensemble de ces variables, toutes ne se sont pas révélées de la même importance par rapport à la problématique. En effet, à la recherche de 
Tableau 1.

Exposition au risque: variables spatialement discriminantes

\begin{tabular}{|c|c|c|}
\hline $\begin{array}{l}\text { Thème } \\
\text { du groupe } \\
\text { de variables }\end{array}$ & Variables & Code \\
\hline \multirow[t]{4}{*}{$\begin{array}{l}\text { Informations } \\
\text { sur le chef de famille }\end{array}$} & $\begin{array}{l}\text { années succ. } \\
\text { passées dans la comm. }\end{array}$ & $\begin{array}{l}\text { durée_1 très peu } \\
\text { durée_2 peu } \\
\text { durée_3 élevées } \\
\text { durée_4 très élevées }\end{array}$ \\
\hline & langue(s) parlée(s) & $\begin{array}{l}\text { CT Castellano/Tacana } \\
\text { C Castellano } \\
\text { E Esse Ejja }\end{array}$ \\
\hline & activité prof. principale & $\begin{array}{l}\text { A+ majorité d'agriculteurs } \\
\text { A bois, agriculteurs } \\
\text { et exploitants de bois } \\
\text { A agriculteurs } \\
\text { Pê pêcheurs }\end{array}$ \\
\hline & autres activités rémun. & $\begin{array}{l}\text { Oact- peu } \\
\text { Oact+ beaucoup }\end{array}$ \\
\hline Bois & usage & $\begin{array}{l}\text { VB vente du bois } \\
\text { FB usage familial du bois } \\
\mathrm{B}(0) \text { pas d'usage de bois }\end{array}$ \\
\hline Santé & accès à un médecin & $\begin{array}{l}\text { doc }=\text { pour certains } \\
\text { avec régularité } \\
\text { doc0 pour personne } \\
\text { quasiment jamais } \\
\text { doc pour tous } \\
\text { régulièrement }\end{array}$ \\
\hline \multirow[t]{2}{*}{ Équipement } & matériaux de la maison & $\begin{array}{l}\text { mota/mader, } \\
\text { motacú et bois } \\
\text { mota/chuch, } \\
\text { motacú et chuchio } \\
\text { chuch, chuchio } \\
\text { mezcla habitat hétérogène }\end{array}$ \\
\hline & $\begin{array}{l}\text { équipement du foyer } \\
\text { (outils agric., biens div.) }\end{array}$ & $\begin{array}{l}\text { équip++ niveau } \\
\text { d'équipement très élevé } \\
\text { équip+ niveau } \\
\text { d'équipement élevé } \\
\text { équip- niveau } \\
\text { d'équipement bas } \\
\text { équip- - niveau } \\
\text { d'équipement très bas }\end{array}$ \\
\hline Activités agricoles & $\begin{array}{l}\text { diversité des animaux } \\
\text { élevés }\end{array}$ & $\begin{array}{l}\text { élev++ diversité élevée } \\
\text { élev+ diversité } \\
\text { élev- diversité faible } \\
\text { élev - - diversité très faible }\end{array}$ \\
\hline
\end{tabular}




\begin{tabular}{|c|c|c|}
\hline \multirow[t]{3}{*}{ Activités agricoles } & $\begin{array}{l}\text { situation des champs } \\
\text { et distance à la maison }\end{array}$ & $\begin{array}{l}\text { dist.tg distance } \\
\text { très grande } \\
\text { dist.g distance grande } \\
\text { dist.f distance faible } \\
\text { dist.tf distance très faible }\end{array}$ \\
\hline & $\begin{array}{l}\text { usage des produits } \\
\text { de l'agriculture }\end{array}$ & $\begin{array}{l}\text { Us.F familial } \\
\text { Us.FV familal prioritaire } \\
\text { et vente } \\
\text { Us.VF vente prioritaire } \\
\text { et familial } \\
\text { Us.F=V familial et vente } \\
\text { égaux }\end{array}$ \\
\hline & $\begin{array}{l}\text { diversité des fruits } \\
\text { et légumes cultivés }\end{array}$ & $\begin{array}{l}\text { Frut+ diversité élevée } \\
\text { Frut.moy diversité moyenne } \\
\text { Frut.- diversité faible }\end{array}$ \\
\hline \multirow[t]{2}{*}{ Chasse } & usage & $\begin{array}{l}\text { Cz.V Vente } \\
\text { Cz.F Familial }\end{array}$ \\
\hline & $\begin{array}{l}\text { diversité des animaux } \\
\text { chassés }\end{array}$ & $\begin{array}{l}\text { Cz.Anim+ élevée } \\
\text { Cz.Anim= moyenne } \\
\text { Cz.- faible }\end{array}$ \\
\hline Cueillette & $\begin{array}{l}\text { diversité des produits } \\
\text { de la cueillette }\end{array}$ & $\begin{array}{l}\text { Cos. +élevée } \\
\text { Cos.= moyenne } \\
\text { Cos.- faible }\end{array}$ \\
\hline \multirow[t]{6}{*}{ Pêche } & $\begin{array}{l}\text { nb. de pers. allant } \\
\text { pêcher dans la famille }\end{array}$ & $\begin{array}{l}\text { Pêà- } 1 \text { personne } \\
\text { Pêà+ à plusieurs } \\
\text { Pêà = variable selon } \\
\text { les familles }\end{array}$ \\
\hline & $\begin{array}{l}\text { diversité des outils } \\
\text { utilisés pour la pêche }\end{array}$ & $\begin{array}{l}\text { Out.pê+ élevée } \\
\text { Out.pê+- moyenne } \\
\text { Out.pê- faible }\end{array}$ \\
\hline & $\begin{array}{l}\text { durée des parties } \\
\text { de pêche }\end{array}$ & $\begin{array}{l}\text { T.pê+ longue } \\
\text { T.pê1 un jour } \\
\text { T.pê- courte }\end{array}$ \\
\hline & achat de poisson & $\begin{array}{l}\text { C.pesc }+ \text { souvent } \\
\text { C.pesc }=\text { de temps en temps } \\
\text { C.pesc- peu }\end{array}$ \\
\hline & $\begin{array}{l}\text { diversité des poissons } \\
\text { pêchés }\end{array}$ & $\begin{array}{l}\text { D+P- diversite forte, } \\
\text { peu de pisciv. } \\
\text { Dmoy P- div. moy., } \\
\text { peu de pisciv. } \\
\text { DP = var. selon familles, } \\
\text { moy. pisciv. } \\
\text { D-P+ div. faible, } \\
\text { beaucoup de pisciv. } \\
\text { D+P+ div. forte, } \\
\text { beaucoup de pisciv. }\end{array}$ \\
\hline & vente de poisson & $\begin{array}{l}\text { VP++ très élevée } \\
\text { VP+ élevée } \\
\text { VP- faible }\end{array}$ \\
\hline
\end{tabular}


Figure 2.

AFC sur les variables issues de l'enquête géographique. 
variables avant tout spatialement discriminantes, un travail de tri à partir de tableaux de fréquence par communauté a permis d'extraire finalement 22 variables, synthétisant parfois plusieurs variables en une, présentant des variations significatives dans l'espace (tabl. 1).

A partir de ces 22 variables, il nous a alors été possible d'extraire une typologie des communautés à partir d'une analyse factorielle des correspondances (AFC). Celle-ci nous a permis de hiérarchiser l'information (qualitative) en regroupant dans un espace factoriel les variables les plus proches les unes des autres, formant des nuages de points constitués par les communautés elles-mêmes et les variables qui les caractérisent.

\section{Résultats: une collaboration qui porte ses fruits}

À partir de l'AFC réalisée, trois axes factoriels principaux ont été dégagés, qui concentrent à eux trois $49,22 \%$ de l'inertie du nuage de points. Le premier axe concentre $21,02 \%$ (valeur propre 0,563 ) de l'inertie du nuage, le deuxième $16,10 \%$ (valeur propre 0,433 ) et le troisième $12,10 \%$ (valeur propre 0,327 ). Les valeurs propres relativement élevées nous confirment une assez forte différenciation entre les individus, cependant les taux d'inertie plutôt faibles indiquent que l'information n'est pas très concentrée sur les axes: certaines variables se retrouvent sur chacun des axes, seules certaines marquent une différence très nette qui permette de les définir.

La forme du nuage de points (fig. 2) permet d'identifier cinq groupes aux modes de vie et pratiques contrastés, malgré les situations similaires des communautés par rapport aux rives du Río Beni. Dès lors, cela laisse supposer que l'exposition au risque de contamination n'est pas égale partout.

L'analyse des coordonnées des individus et des variables sur le premier axe révèle une opposition entre les communautés de pêcheurs aux ressources très peu diversifiées (Groupe 2), et les 
communautés d'agriculteurs de Puerto Motor, Buena Vista, Puerto Yumani et Carmen Soledad (Groupe 3). Le deuxième axe oppose les communautés se dédiant plutôt à la coupe de bois (Groupe 1) aux villages d'agriculteurs (Groupe 3).

Le troisième axe quant à lui permet d'identifier la singularité des communautés de Capaina, Altamarani et Eyiyokivo, où Eyiyokivo demeure tout de même à part.

\section{Groupe 1: \\ une forte diversification des ressources, spécialisation dans l'exploitation du bois}

Le premier groupe est composé des communautés de Villa Fátima, San Marcos, Cachichira, Nuevo Reyes et Soraida. Si elles ne se ressemblent pas en tout point, ces communautés ont de fortes similarités: l'activité principale du chef de famille (agriculture et exploitation de bois) et la vente du bois. Cela les distingue fortement des autres communautés. Ces populations ont recours à la pêche mais les temps de pêche sont courts et le troc ou l'achat de poisson est courant. On note une bonne diversité d'outils de pêche et une diversité de poissons pêchés moyenne. S'ils se dédient majoritairement à l'agriculture et à l'exploitation du bois, ce groupe est caractérisé par une bonne diversification des ressources: fruits et légumes cultivés, cueillette, élevage, chasse semblent s'équilibrer dans leur mode de vie. Par ailleurs, ils possèdent un bon niveau d'équipement, un habitat construit de feuilles de motacú (Scheelea princeps, palmier très commun dans notre espace d'étude) pour le toit et de bois pour les parois (révélateur de cette activité pratiquée).

\section{Groupe 2: une très faible diversification des ressources, spécialisation dans la pêche}

Le deuxième groupe est composé des communautés de Puerto Salinas, Santa Rosita et Maije. Puerto Salinas et Maije sont des communautés dont la population appartient au groupe ethnique des Esse Ejja, connu pour leur mode de vie nomade très lié à la 
pêche (Herrera, 2003). En revanche, Santa Rosita est composée d'une seule famille, ils ne sont pas Esse Ejja, mais semblent avoir adopté un mode de vie très similaire. Il s'agit d'une installation récente, qui peut expliquer la faiblesse de leur équipement. Des processus différents peuvent ainsi conduire à des situations similaires à un moment donné, mais ne laissent pourtant rien présager sur l'évolution possible de leur mode de vie et de leurs pratiques. Cependant à présent, deux ans après l'enquête, une inondation a détruit leurs habitations et cette famille s'est installée à Puerto Salinas même. Le chef de famille en est d'ailleurs devenu le corregidor. Le groupe 2 est caractérisé par un habitat précaire essentiellement constitué de chuchio (Poaceae: Gynerium sagittatum, végétation des rives du cours d'eau), un niveau d'équipement très faible, une diversification des ressources très faible (quasiment pas d'élevage, ni de chasse, ni de cueillette), une faible diversification des cultures, des champs situés à proximité immédiate de l'habitat. Ce groupe semble en revanche se dédier plus à la pêche et ils vendent leur poisson mais en achètent très peu. Enfin, les variétés de poissons pêchés mentionnés sont le plus souvent des poissons piscivores.

\section{Groupe 3 : une bonne diversification, spécialisation dans l'agriculture}

Le troisième groupe est composé de Buena Vista, Puerto Motor, Puerto Yumani et Carmen Soledad. Ce sont des communautés d'agriculteurs. Les produits de l'agriculture sont destinés en majorité à la vente, certainement du fait de la proximité de Rurrenabaque accessible par une piste large, et secondairement à la consommation familiale. La distance à parcourir pour se rendre aux champs est très grande, la diversité de fruits et légumes cultivés est importante. Ils pratiquent abondamment la cueillette et l'élevage. Le niveau d'équipement est très bon. Quant à la pêche, elle n'est généralement l'affaire que d'une seule personne dans la famille, le poisson pêché est très peu vendu, la diversité de poissons pêchés est bonne, mais ils citent peu de piscivores. 


\section{Groupe 4: agriculteurs? pêcheurs ?}

Altamarani et Capaina forment toutes deux un groupe à part entière. En fait, ces deux communautés semblent posséder les caractéristiques des agriculteurs (groupe 3) mais dans une moindre mesure. La diversité d'animaux chassés est grande. Aucune activité dominante ne se détache nettement. En revanche, la pêche semble prendre une importance que l'on ne note pas dans les groupes précédemment cités, à part chez le groupe des pêcheurs (groupe 2). En effet, à Capaina notamment, le poisson est majoritairement vendu et la diversité d'outils employés pour la pêche est grande.

\section{Jeune garçon jetant un filet à Capaina (groupe 4).}

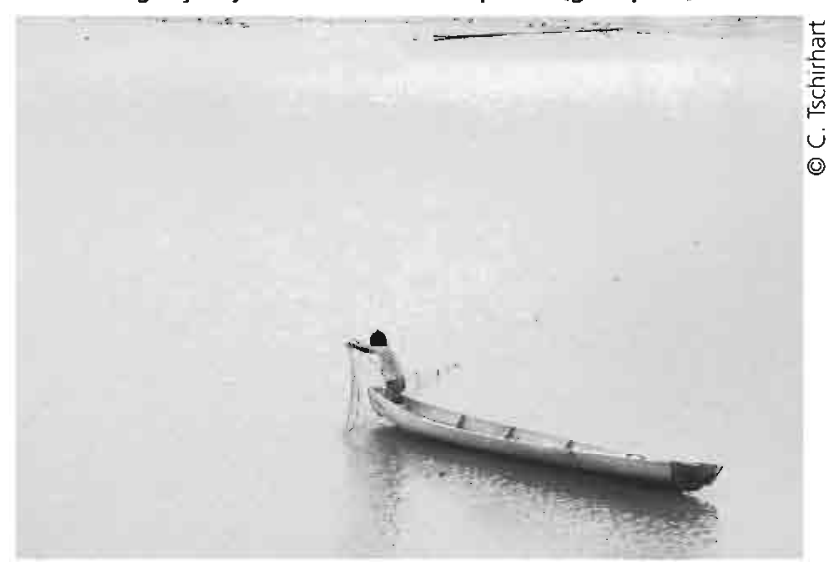

Abris d'une famille Esse Ejja à Puerto Salinas (groupe 2).

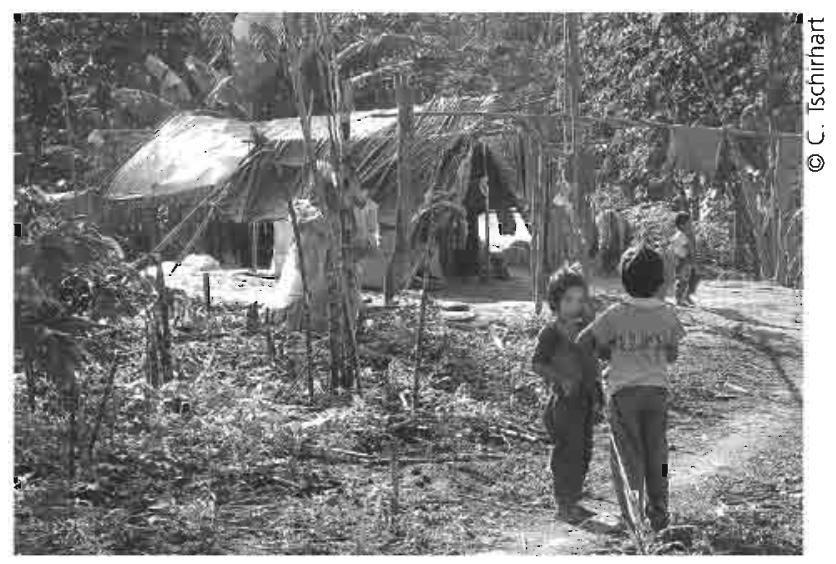


Maison avec parois en bois, toit de tôle

et plaque solaire à Villa Fátima (groupe 1).

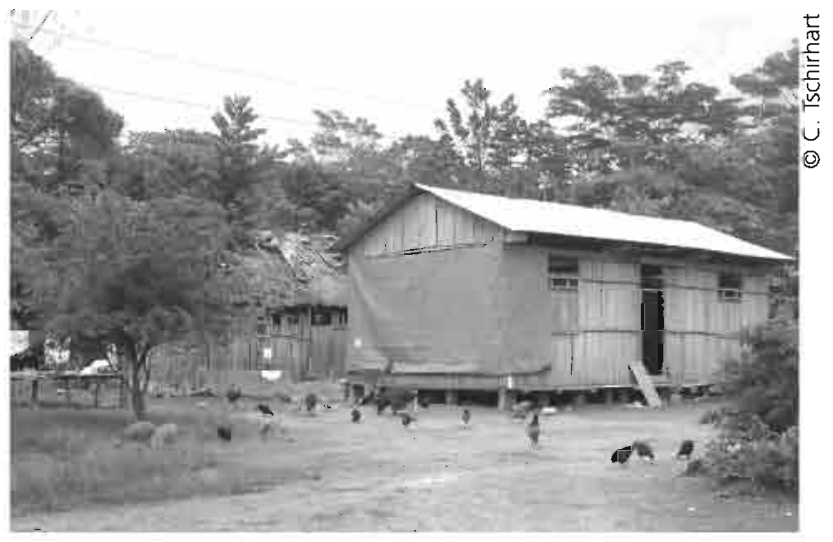

Monoculture de tomates destinées à la vente à Puerto Motor (groupe 3).

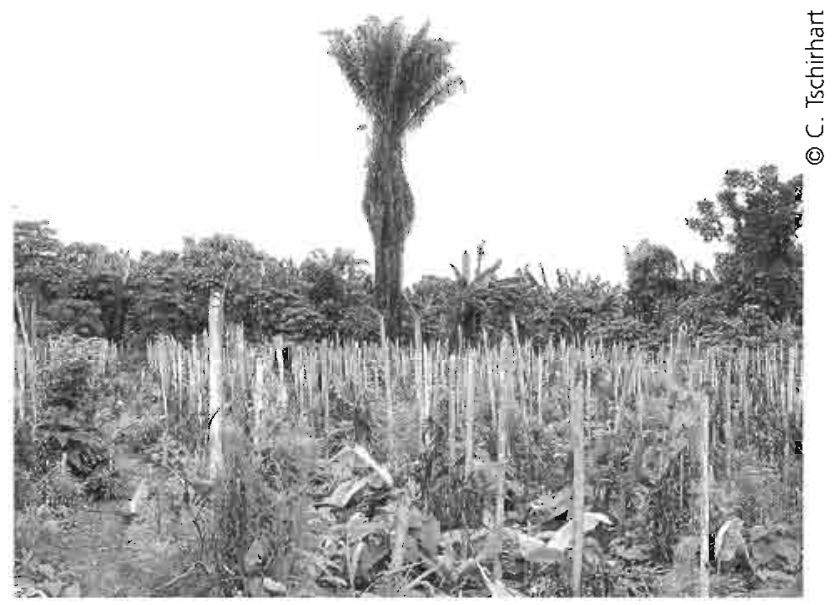

\section{Groupe 5:}

Eyiyokivo, spécialisation dans la pêche, mais diversification des ressources

Cette communauté est nettement caractérisée par une activité dominante: la pêche. L'activité professionnelle mentionnée en majorité est "pêcheur», ils pêchent une grande diversité de poissons et notamment des poissons piscivores, activité pratiquée par plusieurs membres de la famille. Le produit de la pêche est souvent vendu et certaines parties de pêche se révèlent très 
longues (jusqu'à deux semaines). Ce sont également des Esse Ejja, à l'instar du groupe 2. Ils sont d'ailleurs sous beaucoup d'aspects similaires à ce groupe, avec peu d'élevage et peu d'équipement notamment. Ils se détachent cependant du groupe 2 en ayant recours à d'autres activités et en diversifiant leurs ressources, peut-être grâce à la proximité de Rurrenabaque. Ils cultivent fruits et légumes, chassent les animaux de la forêt, cueillent les fruits sylvestres, en fonction de leur disponibilité.

La localisation de ces résultats (fig. 1) révèle une structure spatiale qu'il conviendra de mettre en perspective avec les niveaux de contamination mercurielle.

Les résultats des analyses du niveau de $\mathrm{Hg}$ dans les cheveux sont pour le moment provisoires car selon les protocoles établis ils doivent être soumis à une deuxième lecture, qui validera la première. Les données chiffrées sont susceptibles de subir de légers ajustements et ne peuvent donc être analysées à l'échelle des communautés. En revanche, les ordres de grandeur, communiqués par l'équipe biomédicale avec ces réserves, peuvent être mis en perspective avec la typologie géographique.

Les niveaux de contamination sont faibles dans l'ensemble, puisque seuls $10 \%$ de la population enquêtée environ (femmes et enfants) se situent au-dessus du seuil des $10 \mu \mathrm{g} / \mathrm{g}$. Donc $90 \% \mathrm{de}$ la population aurait des valeurs inférieures au seuil de $10 \mu \mathrm{g} / \mathrm{g}$, et $60 \%$ auraient des valeurs inférieures à $5 \mu \mathrm{g} / \mathrm{g}$, c'est-à-dire en dessous du seuil d'innocuité.

Si l'on distribue la contamination par le MeHg selon 3 classes, faible, moyenne, élevée, on obtient les résultats suivants:

Groupe 3: faible

Groupes 1, 4 et 5: moyen

Groupe 2: fort

Une plus grande précision chiffrée ne peut être obtenue pour l'instant au vu du caractère provisoire des résultats mercuriels. En revanche, ils révèlent dès à présent le lien entre la contamination par le mercure et la gestion de l'espace à l'échelle communautaire. 


\section{Discussion}

Ces premiers résultats confirment que les populations riveraines du Río Beni ne sont pas identiques et ont développé des modes de vie différents. Pourtant, ces communautés ont une situation quasiment identique par rapport à la rivière, et ce dans un espace assez restreint (distance d'environ $100 \mathrm{~km}$ entre Rurrenabaque et Villa Fátima). Enfin ces populations possèdent une culture commune (Tacana), seules les populations Esse Ejja se démarquant nettement.

Le recours à différentes ressources est de règle dans quasiment toutes les communautés, jonglant essentiellement entre l'agriculture, la chasse, la pêche et la cueillette, puis quelques autres activités rémunératrices (construction de maison, construction de bateaux, ouvrier agricole...). C'est cependant l'importance relative qu'elles occupent dans l'ensemble des activités qui crée le contraste. Ainsi, le groupe 3 se dédie plus à l'agriculture, le groupe 1 à l'exploitation du bois, le groupe 5 à la pêche.

Les résultats obtenus sur les pratiques et modes de vie soulèvent un premier questionnement sur le rôle que joue la distance à San Buenaventura et Rurrenabaque sur le niveau d'exposition au risque de contamination et sur les modes de vie. En effet, la communauté d'Eyiyokivo (groupe 5) est une communauté de pêcheurs a priori très exposée au risque de contamination, puisque les opportunités d'y consommer du poisson y sont élevées. Pourtant les niveaux de contamination observés d'après les premières analyses la situent dans le groupe intermédiaire. Il est probable qu'une forte proportion des poissons pêchés, à valeur commerciale élevée (les poissons situés en bout de chaîne trophique), soit réservée à la vente, les autres (poissons de petite taille et herbivores) étant attribués à la consommation familiale, qui constitue alors un facteur protecteur pour cette communauté. Le poisson à forte teneur en mercure est ainsi dilué dans la population de la ville de Rurrenabaque, la proximité de cette ville facilitant la commercialisation du poisson, via le Río Beni ou via une piste entrant directement dans la communauté et réalisée à cet effet. Rurrenabaque possède par ailleurs un nombre important de restaurants en raison de la vocation touristique de la région permettant 
d'ébaucher l'idée d'une dilution de la ressource contaminante dans une population nombreuse et non durablement exposée, réduisant considérablement le risque sanitaire, cette hypothèse restant bien évidemment à valider ultérieurement. Néanmoins Eyiyokivo étant constituée d'une population très mobile, il est possible que les individus et familles qui ont pu être enquêtés au sein de cette communauté ne soient pas réellement représentatifs de l'ensemble de la population.

En ce qui concerne le groupe 4, constitué des communautés de Capaina et Altamarani, le rôle de la proximité de ces deux villes est plus ambigu. Ce sont des communautés Tacana, aux ressources diversifiées. Cependant le poisson est majoritairement dédié à la vente et de manière générale les variables liées à la pêche montrent que cette activité prend une place relativement importante dans les pratiques des communautaires, surtout à Capaina. La question suivante peut être soulevée: ne serait-ce pas la proximité de Rurrenabaque et San Buenaventura et la facilité de commercialisation du poisson qui auraient encouragé le développement de cette activité, incitant ainsi à une augmentation de la consommation de poisson et les exposant plus au risque de contamination? Cela reste également à investiguer.

Nous émettons l'hypothèse que les accès différenciés aux ressources alimentaires et leurs gestions peuvent être révélés par des indicateurs paysagers traduisant la relation au Río Beni et donc à la ressource poisson contaminante. En effet, l'éloignement des parcelles de culture par rapport aux habitations et leur situation (à proximité du cours d'eau, en forêt, à proximité de l'habitation...) sont des révélateurs de la place qu'occupe le río dans les économies familiales. Comme nous l'avons vu, le groupe 2 possède ses maigres cultures à proximité immédiate des habitations, ou même sur une île en face de la communauté dans le cas d'Eyiyokivo (groupe 5), tandis que les champs du groupe 3 se situent à des distances importantes (jusqu'à 1 h 30 de marche). De plus, les communautés composant ce dernier type (Buena Vista, Carmen Soledad, Puerto Yumani et Puerto Motor) sont parmi les seules à ne pas posséder d'ouverture directe sur le Río Beni. Cela est en partie dû à la volonté de protéger les cultures des fortes inondations, surtout en rive droite. Il a également été mentionné la voracité des porcs comme motif d'éloignement des cultures. Analysées de 
front avec les activités dominantes, ces variables semblent donc constituer un indicateur de l'importance que prend le Río Beni dans les pratiques, forte pour les groupes 2 et 5 , intermédiaire pour les groupes 1 et 4 et faible pour le groupe 3 dans lequel la pêche apparaît seulement comme une activité de loisir permettant de diversifier l'alimentation. Dans ce dernier groupe, c'est l'agriculture qui prend une place essentielle dans le mode de vie et les produits de l'agriculture semblent constituer une source de revenus importante, grâce à leur commercialisation au marché de Rurrenabaque. Cette diversité pourrait ainsi constituer un ensemble de facteurs protecteurs expliquant la faiblesse de la contamination. L'espace pratiqué par les groupes identifiés serait d'un côté plus terrestre et de l'autre plus fluvial: cette pratique de l'espace pourrait-elle être un des facteurs influençant l'alimentation et donc l'exposition à la contamination par le $\mathrm{Hg}$ ? Il sera néanmoins important de mesurer l'importance du recours à la ressource poisson dans les lagunas (bras morts du fleuve), qui sont situés à l'intérieur des terres, et sont des lieux privilégiés de méthylation du $\mathrm{Hg}$.

La proximité au Río Beni n'entraîne pas nécessairement des niveaux de contamination élevés comme l'illustrent les groupes 1 et 2, le risque variant selon l'activité dominante. Ainsi le groupe 1 (San Marcos, Villa Fátima, Cachichira, Soraida et Nuevo Reyes) se consacre majoritairement à l'exploitation du bois pour les revenus monétaires qu'elle procure, puis a recours à un ensemble de ressources, destinées à la consommation familiale essentiellement, dans lequel le poisson ne semble pas occuper une place majeure. Cependant en raison de l'éloignement des marchés, les poissons ne sont pas vendus (ou extrêmement peu) et donc consommés au sein des familles, qu'il s'agisse de poissons de début ou de fin de chaîne trophique. La consommation de poissons de fin de chaîne trophique et la place périphérique du poisson dans les menus des familles de ce groupe, en raison des activités d'extraction du bois et de la diversité des autres ressources, peuvent expliquer les niveaux de contamination moyens des communautés qui le compose. La diversification des ressources et la spécialisation dans d'autres activités constitueraient ainsi des facteurs protecteurs en réduisant la place de la consommation de poissons pourtant $a$ priori fortement contaminés. 
En revanche, le groupe 2, qui présente les niveaux de contamination les plus élevés, a peu recours aux autres ressources disponibles (chasse, pêche, cueillette) et a très peu développé et diversifié la pratique de l'agriculture. Le mode de vie précaire des habitants, qui s'observe autant dans l'habitat que dans les biens possédés, est révélateur d'un certain détachement à la terre, d'un mode de vie plus nomade et orienté vers le rio. Le poisson semble en premier lieu dédié à la vente alors que l'achat de poisson est peu courant. La pêche occupe donc une place importante dans le mode de vie de ces communautés. Mais le temps nécessaire pour rejoindre Rurrenabaque et San Buenaventura (environ 6 heures en pirogue à petit moteur peque-peque) ne permet pas une commercialisation aussi poussée du poisson qu'à Eyiyokivo. La consommation de poisson contaminé est alors plus élevée et explique probablement la plus forte contamination: une faible diversification des ressources ainsi que l'éloignement des centres marchands semblent ainsi constituer les principaux facteurs de risque.

\section{Conclusion}

Quelques limites se doivent d'être soulignées dans le cadre de cette étude. Tout d'abord, le problème des effectifs constitue l'un des problèmes majeurs rencontrés, surtout en vue d'un traitement quantitatif. En effet, les effectifs de familles enquêtées par communauté sont faibles et ne dépassent les 30 que dans une communauté (Buena Vista). 11 a donc été particulièrement délicat de travailler à partir des valeurs relatives pour comparer les communautés entre elles; on aura souvent préféré travailler sur les effectifs pour créer les profils des communautés. Ce constat est illustratif des difficultés rencontrées dans le montage de projets pluridisciplinaires. En effet, le coût prohibitif des analyses biologiques permettant le diagnostic de contamination et la mesure du taux de mercure présent dans les cheveux oblige les équipes biomédicales à réduire les effectifs étudiés pour rester dans l'enveloppe financière disponible. En raison des objectifs spécifiques d'une approche biomédicale, cette contrainte n'est pas nécessairement handicapante dans la mesure où les effectifs individuels 
sont suffisamment élevés par rapport aux méthodes statistiques utilisées. En revanche, dans le cadre d'un diagnostic de type communautaire comme nous ambitionnons de le faire, il est évident que cette limite nous oppose de fortes contraintes quant à la portée des résultats obtenus.

En se référant à la comparaison évoquée plus haut entre les travaux menés sur l'onchocercose et les nôtres, il est bien évident qu'une lacune forte apparait dans le fait d'avoir limité les communautés enquêtées au bord du rio à l'exclusion de communautés situées dans des lignes de risque théoriquement plus faible car plus éloignées de la ressource contaminante. Seules les communautés de Carmen Soledad, Puerto Yumani, Puerto Motor et Nuevo Reyes se trouvent plus en retrait du rivage. Les contraintes, notamment financières, évoquées plus haut mais également les contraintes de temps et de force de travail disponibles expliquent cette limite. Ainsi, l'utilisation de calepins, questionnaires, crayons et pataugas permet d'envisager des extensions de l'enquête géographique sans surcoût notable contrairement aux investigations biologiques. Cependant, cette extension se heurterait à une autre exigence des projets de recherche pluridisciplinaire qui consiste à harmoniser les échantillons en termes d'échelle et d'effectifs afin de pouvoir établir la mise en perspective entre les différents corpus de données. Au-delà de la prise en compte d'un système dans sa globalité et ses interrelations qui sont à la base de la justification de la mise en œuvre de la pluridisciplinarité, la réalisation méthodologique de terrain oppose ainsi des contraintes fortes dont il est délicat de s'affranchir.

Pour conclure, les résultats biologiques attendent leur $2^{\mathrm{e}}$ lecture de confirmation, mais de nombreuses hypothèses fortes ont déjà pu être posées à partir de l'analyse qualitative menée sur les facteurs protecteurs et les facteurs de risque et pourront être mesurées à partir des données individuelles chiffrées.

Cette approche géographique a été développée pour répondre à un questionnement en santé publique. L'objectif final (connaître les déterminants de la construction des espaces et des pratiques à risque à partir de la mise en évidence des facteurs de risques) a conduit à la mise en évidence de territoires aux structurations et aux modes de gestion hétérogènes. Leur mise en relation avec les 
classes de contamination montre que le niveau de $\mathrm{Hg}$ dans les cheveux pourrait devenir un indicateur des modes de vie, des pratiques spatiales et de la gestion des ressources.

La prochaine étape de cette étude aura pour objectif de mesurer les déterminants du risque d'exposition, de contamination, et de mettre en évidence la diversité intravillageoise, permettant d'illustrer le poids des pratiques individuelles au sein d'un espace collectif. Cela nécessitera alors le passage à une échelle plus fine. C'est à cette échelle que pourront être évalués les modes d'accès aux ressources alimentaires et leur gestion, les modes d'accès aux structures de soin, les modes d'accès à l'éducation et la scolarisation, les mobilités des populations, les filières de commercialisation du poisson dans toutes leurs spécificités et variabilités. Des cartes des parcours de vie, afin de connaître les aires de rayonnement des communautés, de connaître l'ouverture vers l'extérieur, les éventuels réseaux présents, seront alors réalisées.

Ces premières analyses prometteuses semblent confirmer l'existence d'une relation entre modes de vie et contamination par le $\mathrm{MeHg}$. Le risque lié à la contamination du milieu est cependant dépendant de la gestion des ressources par les sociétés en fonction de leur maîtrise des territoires et des réseaux. Les sociétés et les lieux, de par leurs spécificités, modulent ce risque. L'approche systémique et interdisciplinaire appliquée ici livre dès maintenant des pistes intéressantes pour des niveaux de contamination pourtant plutôt faibles. Les analyses à suivre nous permettent d'envisager des propositions en matière de développement et d'aménagement des espaces riverains des rios de l'Amazonie bolivienne afin de limiter les risques de contamination des habitants.

\section{Remerciements}

Cet article n'aurait pu paraitre sans l'équipe santé de l'UR 024 (IRD) présente en Bolivie: merci à Éric Bénéfice, Selma Luna Monroy, Ronald Lopez et Aurélien Radufe pour la collecte et le traitement des données mercurielles ainsi que pour la communication des premiers résultats de niveaux de $\mathrm{Hg}$ dans les cheveux malgré toutes les réserves existantes. Ces mesures de taux de contamination dans les cheveux ont été réalisées au laboratoire de Qualité environnementale de l'Institut d'écologie (Cota Cota, La Paz, Bolivie) par Marc Roulet et Jaime Chincherro que nous remercions très chaleureusement.

Cet article est dédié à la mémoire de Marc Roulet. 


\section{Références bibliographiques}

CARMOUZE J.-P., LuCOTTE M., Boudou A., 2001 - Le mercure en Amazonie. Rôle de l'homme et de l'environnement, risques sanitaires. Paris, IRD Editions, Exp. Coll., 494 p.

Dumont J.-F, Hérail G., GuYot J.-L., 1991 - « Subsidencia, inestabilidad y repartición de los plaeres distales de oro. El caso del Río Beni (Bolivia)». In Hérail et Fornari, éd. : Simposio internacional sobre los yacimientos de oro, La Paz, Bolivia, 3-5 junio 1991, Orstom Éd. : 43-46.

HANDSCHUMACHER P., HERVOUËT J.-P., 2004 - Des systèmes pathogènes à la santé publique: une nouvelle dimension pour la géographie de la santé tropicale. Autrepart, 29: 47-63.

HerRera E., 2003 - Etnografia del aprovechamiento de la fauna icticola en una comunidad Ese Ejja de reciente sedentarización. La Paz, Tesis de Lic., Antropología, Universidad Mayor de San Andres.

HERVOUĖT J.-P., 1990 - Le mythe des vallées dépeuplées par l'onchocercose. Géos $\mathrm{n}^{\circ} 18,35 \mathrm{p}$.

JENSEN S., Jornelov A., 1969 - Biological methylation of mercury in aquatic organisms. Nature $n^{\circ} 223: 753-754$.

LEBEL J. et al., 1998 - Neurotoxic effects of low-level methylmercury contamination in the Amazonian Basin. Environ. Res., $79: 20-32$.

Maurice-Bourgoin L. et al., 1999 - Mercury Pollution in the upper Beni River, Amazonian Basin, Bolivia. Ambio, vol. 28, n 4 : 302-306.

MAURICE-BOURGOIN L. et al., 2000 - Mercury distribution in waters and fishes of the upper Madeira rivers and mercury exposure in riparian Amazonian populations. Elsevier, $\mathrm{n}^{\circ}$ 260: 73-86.

Maurice-Bourgoin L., 2001 - El mercurio en la Amazonia boliviana. Bolivia, La Paz, Editorial Offset Boliviana Ltda. EDOBOL, 75 p.

MURESAN PASLARU B., 2006 - Géochimie du mercure dans le continuum de la retenue de Petit-Saut et de l'estuaire du Sinnamary, Guyane française. Thèse de doctorat en Biogéochimie de l'environnement, Bordeaux-I, 267 p.

SALEM G., 1998 - La santé dans la ville - Géographie d'un petit espace dense (Pikine, Sénégal). Karthala/Orstom, 360 p.

WHO, 1990 - Environmental Health Criteria 101: Methylmercury. Geneva, World Health Organisation, International Programme on Chemical Safety, 144 p. 



\section{Itinéraires des piétons et risque d'exposition à la pollution en zone urbaine: approche méthodologique}

\section{Gilles MAIGNANT}

Jérôme DUTOZIA

Le paradigme de complexité invite à repenser les interactions qui unissent les différentes facettes et les différents angles d'observation d'un même objet, on admet alors qu'aucune science ne dispose d'objets propres, chaque discipline donne un point de vue propre sur des objets que d'autres disciplines peuvent envisager (CHARRE, 2003). De plus, la complexité est invisible dans des disciplines qui fragmentent l'objet ou qui l'isolent. D'où la nécessité de relier. Car dès que vous avez un objet riche multidimensionnel, il ne suffira pas d'additionner les disciplines (MORIN, 2002). Pour reprendre une expression de Basarab Nicolescu, un autre théoricien de la transdisciplinarité, l'appréhension des objets complexes passe par « un big bang disciplinaire", dont l'enjeu est de transformer une pensée pluridisciplinaire - ce à quoi Morin fait référence quand il évoque l'insuffisance d'additionner les disciplines - en une pensée transdisciplinaire - à la fois entre, à travers, et au-delà des disciplines, (NiCOlesCu et al., 1994).

Du fait de sa position au cœur de l'interdépendance entre l'homme et son milieu de vie, ainsi que de la diversité des facteurs et cofacteurs 
qui la déterminent, la santé est un objet de recherche qui suscite de nombreuses collaborations entre les disciplines. Mais la singularité de chacune des disciplines est à la fois la condition de leurs complémentarités, et la source de leurs contradictions, de leurs incompréhensions et de leurs désaccords méthodologiques. Dans l'appréhension transdisciplinaire des enjeux de santé, il y a donc besoin de terrains d'entente, de points de rencontre qui permettent de croiser les nombreuses dimensions de l'interaction entre environnement et santé. L'espace est un de ces points de rencontre.

Notre travail n'a pas pour objectif de chercher à comprendre des règles de répartition spatiale d'un phénomène sanitaire ou à identifier des co-variations spatiales entre des facteurs environnementaux et l'état de santé des populations. Nous cherchons plutôt à modéliser un système de risque sanitaire dans un contexte de déplacement pédestre, système ayant une dimension pluridisciplinaire et complexe, puisqu'il cherche à englober des facteurs biologiques (vulnérabilité des individus), morphologiques (formes de la ville et du réseau urbain), environnementaux (pollution de l'air) et sociologiques (perception du niveau de pollution et acceptabilité d'un itinéraire de déplacement différent de l'optimum distance/temps). La méthodologie se veut opérationnelle et orientée vers les besoins de la société, puisqu'elle devra permettre de proposer, aux usagers de la ville, des itinéraires alternatifs à l'optimum distance/temps, permettant aux populations d'adapter leurs itinéraires afin de minimiser la pression environnementale subie au cours de leurs déplacements pédestres.

Dans un contexte de déplacements urbains qui aujourd'hui privilégie la marche ou le vélo, il nous semble pertinent d'évaluer les itinéraires qui s'offrent au piéton en y intégrant un facteur supplémentaire: l'environnement. En effet, traditionnellement, les itinéraires sont estimés selon des contraintes temporelles ou spatiales; ces facteurs ont bien évidemment leur importance mais ils sont insuffisants. S'il est assez facile de mesurer le temps nécessaire à parcourir un arc d'un réseau urbain en fonction de sa morphologie (sinuosité, pente, etc.) ou de calculer sa longueur, il est en revanche plus délicat d'affecter à chaque arc un poids en fonction de l'environnement. 
Nous nous intéresserons ici seulement à l'un des facteurs constituant l'environnement urbain à savoir la pollution de l'air, qui, avec le bruit, est le facteur qui interagit le plus sur la qualité de vie (BOITEUX, 2001).

\section{Réaménagement de la ville et pollution de l'air: la transformation du milieu de vie et ses impacts sur la santé des habitants}

Dans un contexte de densification et de pression urbaine forte, les lois françaises sur le renouvellement urbain incitent la gouvernance territoriale à mettre en cuvre une politique visant à combler les vides interstitiels du tissu urbain existant afin d'en limiter l'étalement. Les modifications suscitées par ces lois sur la morphologie des villes interagissent sous différentes formes et par le biais de différents phénomènes avec la qualité du milieu de vie et la santé des populations. Parmi ces interactions, nous nous intéresserons ici à la charge polluante respirée par les piétons se déplaçant sur la trame viaire selon différents itinéraires pédestres.

La pollution de l'air, en ville, est essentiellement produite par la circulation automobile, mais les concentrations de polluants peuvent varier considérablement à l'échelle de la ville, voire même d'un quartier. Les immissions de polluants (la charge polluante respirée par les citadins) sont dépendantes des émissions produites sur les axes urbains et des conditions plus ou moins favorables de dispersion. La ville, de par sa forme complexe, agit sur la dispersion des polluants (MAIGNANT, 2002). En effet, la morphologie urbaine, prise dans sa double acceptation, verticale et horizontale, guide les écoulements de champs de vent, champs tantôt accélérés, tantôt freinés. Les interstices entre bâtiments sont nécessaires à la dispersion des polluants émis par la circulation automobile sur les axes. Occulter complètement cette caractéristique des "espaces vides", c'est prendre le risque de faire naître des zones propices à l'accumulation de polluants (rues dites en canyons); phénomène d'autant plus 
pénalisant si la rue est étroite, puisque, pour une circulation automobile donnée, plus la rue est étroite, plus les concentrations sont dépendantes de la morphologie urbaine; et plus la rue est large, plus les concentrations sont dépendantes du vent (vitesse et direction).

Le problème de la pollution de l'air produite par la circulation urbaine est très complexe. En effet, les villes supportent des flux de circulation automobile de plus en plus importants et n'ont pas été conçues pour cela. Depuis «le choc de l'automobile" de la période 1950-1960, les différentes percées effectuées par les aménageurs dans la morphologie urbaine avaient comme objectif premier l'amélioration de la fluidité du trafic au détriment de préoccupations environnementales et sanitaires. De plus, la forte pression foncière que subissent les centres-villes incite les promoteurs et les aménageurs à reconstruire la ville sur la ville, c'està-dire non seulement combler les vides interstitiels mais aussi surélever les bâtiments créant ainsi des pièges remarquables à polluants. Rappelons simplement que l'un des objectifs des réaménagements urbains (percées dans la morphologie, élargissement de la voirie) prônés par le baron Haussmann était de lutter contre l'insalubrité du milieu urbain afin d'améliorer la qualité de vie, le bien-être et la santé des habitants. Les transformations urbaines actuelles vont à l'encontre de celles du passé et modifient aussi d'autres facteurs environnementaux constitutifs de la qualité de vie comme l'ensoleillement ou les nuisances sonores.

Les piétons constituent une population fortement exposée aux flux de polluants des automobiles, et cet enjeu est d'autant plus essentiel pour peu qu'on considère la sensibilité et la vulnérabilité accrue des enfants et des personnes âgées qui représentent une part importante des populations piétonnes.

\section{Vulnérabilité et système de risque sanitaire dans un contexte de déplacements pédestres}

Habituellement quand on parle de risque, on conçoit l'aléa comme un événement brutal, un élément déclencheur du système de risque dont on parvient plus ou moins bien à évaluer la probabilité 
d'occurrence. Or, cette définition restrictive est un cadre de pensée qui exclut de nombreux types d'aléas, entre autres la pollution urbaine pour les risques sanitaires. En prenant en compte la variabilité des temporalités et des rythmes d'un système de risque, les notions telles que celles de "catastrophes au ralenti» (ROQUEPLO, 1987), d'aléa continu ou encore de risque latent sont particulièrement adaptées pour décrire les processus de risque sanitaire, et plus particulièrement ceux liés à une exposition plus ou moins longue à une pression environnementale quelle qu'elle soit.

Le risque se définit comme la rencontre entre un aléa et des enjeux plus ou moins vulnérables. S'agissant de risques sanitaires, nos enjeux sont exclusivement humains, il s'agit des piétons qui vont chercher à rejoindre un point $\mathrm{B}$ à partir d'un point $\mathrm{A}$, et qui vont être soumis à une pression environnementale plus ou moins importante en fonction de l'itinéraire qu'ils vont emprunter (variabilité spatiale de l'intensité de l'aléa). Au niveau des enjeux, il nous paraît important de considérer la différenciation des niveaux de vulnérabilité.

Notre système de risque est complexifié par ce souci de considérer la vulnérabilité différenciée des piétons. En effet, on sait qu'un même événement aura un impact différent selon l'état de santé, l'âge et les spécificités biologiques des personnes. Nous avons donc pris le parti de différencier trois niveaux de vulnérabilité (sensibilité faible, modérée ou forte), en fonction desquels l'exposition à la pression environnementale va avoir un impact plus ou moins fort sur la santé des individus. Pour un même itinéraire, et donc une exposition équivalente, les populations vulnérables, que sont les enfants ou les personnes âgées par exemple, vont donc être davantage affaiblies, que des personnes ne répondant pas à des critères de vulnérabilité particuliers.

En proposant une comparaison entre les itinéraires optimaux en termes environnementaux et les itinéraires optimaux en termes de distance, il ne s'agit pas pour nous de réduire la sélection des itinéraires pédestres à ces deux seuls facteurs ${ }^{1}$. À dire vrai, comprendre

1 Pour un état de l'art récent sur l'ensemble des critères qui ont une influence sur les piétons dans leurs choix d'itinéraires urbains, on pourra se référer aux travaux de Sonya Lavadinho et Guiseppe Pini de l'Observatoire universitaire de la mobilité de Genève, qui identifient plus de 25 caractéristiques ayant un impact potentiel sur les choix d'itinéraires pédestres (PINI et LAVADINHO, 2005). 
ce qui pourrait inciter un individu à emprunter une artère plutôt qu'une autre n'est pas le sujet de notre étude. Nous faisons simplement l'hypothèse que si une méthode est capable de détecter un itinéraire minimisant le niveau d'exposition aux flux polluants, et que cet itinéraire optimal est relativement proche de l'optimal distance/temps, alors ce tracé sera considéré comme acceptable pour une partie de la population. Ce seuil d'acceptabilité n'est pas fixe mais peut varier en fonction du piéton, de ses pratiques, des caractéristiques et des finalités de son déplacement. D’autre part, au niveau de la différence entre les tracés des itinéraires optimaux en distance (OD) et des itinéraires optimaux en termes d'environnement (OE), il ne faut pas négliger l'importance du temps de déplacement (qui est aussi le temps d'exposition), intégré dans notre formulation de l'indicateur d'exposition à travers le facteur de la longueur de l'itinéraire. C'est-à-dire que la recherche de l'itinéraire optimal en termes d'environnement est forcément sensible à la longueur du trajet, ce qui va limiter sa différenciation avec l'optimum distance. L'itinéraire optimal environnemental est donc la recherche d'un compromis minimisant à la fois le niveau d'exposition mais aussi le temps d'exposition (et donc la longueur du parcours).

Dans notre travail, nous avons volontairement simplifié l'application en considérant que quels que soient leurs niveaux de vulnérabilité, les populations se déplacent à une vitesse identique et constante. Ainsi, le temps d'exposition varie seulement en fonction de la longueur des artères. Si nous avions considéré différents types de déplacements pédestres, en distinguant par exemple un mode "marche" et un mode "course», le système modélisé aurait été beaucoup plus complexe de par les interactions fortes entre vitesse de déplacement, temps d'exposition et volume d'air inhalé. En course, un individu se déplace environ à $15 \mathrm{~km} / \mathrm{h}$ alors qu'il ne se déplace qu'à $5 \mathrm{~km} / \mathrm{h}$ en marchant, le coureur va donc réduire nettement son temps d'exposition; mais son niveau global d'exposition aux polluants ne baissera pas nécessairement, car la baisse du temps d'exposition, qui pourrait être calculée dans notre indicateur en pondérant la distance par une valeur inférieure à 1 , sera compensée par la hausse importante de son niveau de vulnérabilité puisqu'un individu courant inhale entre 60 et 100 litres d'air par minutes alors qu'un individu marchant n'inhale pas plus de 15 litres d'air par minute. 


\section{Logique floue et perception du niveau de pollution des artères urbaines}

La première difficulté est d'établir une méthodologie pour estimer si le chemin parcouru est sain ou "nuisible" pour la santé. Pour cela, deux approches s'offrent à nous. La première est la méthode binaire: l'arc est pollué ou non, la catégorisation s'effectuant en fonction des valeurs guides ${ }^{2}$ fixées par l'OMS (OMS, 2000); l'inconvénient de cette méthode réside dans la valeur du seuil qui ne peut être considéré comme un changement d'état. En effet, cela revient à regrouper dans la même classe l'absence de pollution et une concentration proche de la valeur guide, ce qui est d'autant plus ardu que certains constituants chimiques de l'atmosphère sont nécessaires à la vie mais néfastes à des concentrations élevées. Cette complexité vient aussi du fait que le niveau de pollution est aussi variable en fonction des moments de la journée. La deuxième approche est la méthode dite de la logique floue. Elle consiste à introduire un degré de vraisemblance dans la catégorisation d'un arc en pollué ou sain. Rappelons brièvement quelques éléments de logique floue.

La logique floue a été introduite par L. ZADEH (1965) pour réduire les coûts de développements de logiciels. Ce concept de logique floue a été développé pour faire face à l'incapacité de classer des phénomènes en deux groupes: vrai ou faux (variables booléennes), la plupart des phénomènes réels devant être appréhendés par une variation progressive. La logique floue repose sur une modélisation des entrées d'un système par des courbes décrivant les degrés d'appartenance aux différents états identifiés des entrées. Les différents degrés d'appartenance se composent selon deux logiques: la logique «ou» et la logique «et». Dans le cadre de la logique "ou", on considère le degré d'appartenance maximal parmi les conditions d'entrée, tandis que dans le cadre de la logique «et» on considère le degré d'appartenance minimal. Le choix du degré

2 Valeurs guides: les valeurs guides sont des valeurs dont les dépassements éventuels ne représentent que des indicateurs de la réalité du problème et sont des objectifs de santé publique à atteindre. 
d'appartenance maximal et minimal est similaire à celui de la théorie des intervalles. En effet, lorsque l'on cherche l'ensemble des éléments qui appartiennent à l'un ou l'autre des deux intervalles, cela signifie l'union des intervalles soit l'ensemble maximum, tandis que pour les éléments qui appartiennent à l'un et à l'autre des deux intervalles, cela signifie l'intersection et donc l'intervalle minimal. La logique floue est basée sur les ensembles flous (fonction d'appartenance), tandis que la logique classique est basée sur la fonction d'appartenance $\left[\mu_{\mathrm{A}}(X)\right]$ d'un ensemble classique autrement appelé le symbole de Kronecker (tabl. 1).

Tableau 1.

La logique floue basée sur la fonction d'appartenance

\begin{tabular}{|lcc|}
\hline $\begin{array}{l}\text { Fonction } \\
\text { d'appartenance }\end{array}$ & $\begin{array}{l}\text { Logique } \\
\text { classique }\end{array}$ & $\begin{array}{c}\text { Logique } \\
\text { floue }\end{array}$ \\
\hline$\mu_{A}(X)$ & 0 ou 1 & $\in[0,1]$ \\
\hline
\end{tabular}

La logique floue est particulièrement bien adaptée à l'appréhension des itinéraires urbains sous contraintes environnementales. En effet, un citadin, fortement sensibilisé aux enjeux de santé et d'environnement, qui souhaite se rendre d'un endroit à un autre cherchera à optimiser son parcours environnemental, c'est-à-dire emprunter les axes les moins pollués possibles, le niveau de pollution de chaque axe variant de manière continue de 0 à 1 ( 0 signifiant un axe non pollué, 1 , un axe irrespirable ${ }^{3}$ hautement pollué). Bien entendu dans ce type d'analyse, on admet que l'usager a une bonne connaissance des niveaux de concentrations de polluants des axes, ce qui probablement deviendra bientôt une réalité, notamment grâce à la publication des cadastres d'émissions des villes et des panneaux d'information en temps réel qui tendent à se généraliser dans les grandes villes. Paris dispose à ce jour de 170 panneaux lumineux à messages variables, implantés dans les rues, donnant une information en temps quasi réel sur la qualité de l'air.

\footnotetext{
3 Un axe irrespirable est défini comme un axe dépassant de manière notoire les seuils réglementaires.
} 
Figure 1.

Principe de la logique floue: modalités et fonctions d'appartenance.

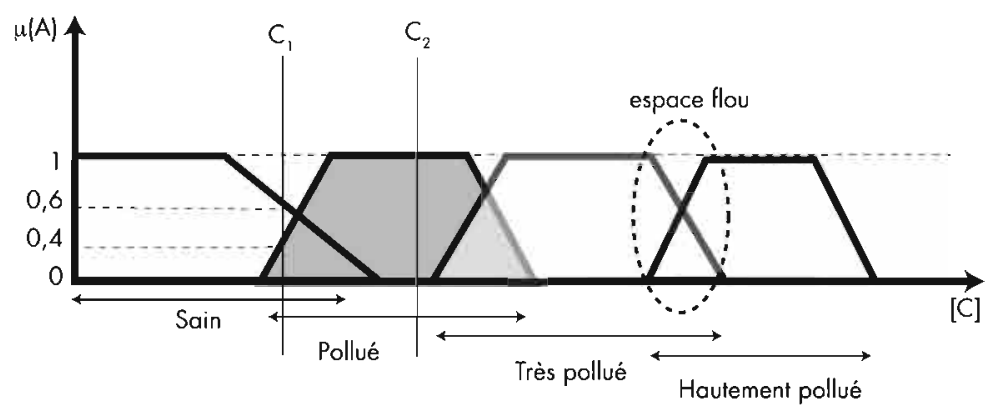

Le concept de logique floue permet d'introduire des nuances dans la perception de la pollution des artères urbaines par le citadin. En effet, elle est le seul moyen de traiter, dans un même cadre, des connaissances fournies numériquement par des instruments de mesure et des connaissances exprimées symboliquement par un observateur humain (BOUCHON-MEUNIER, 1993). Si l'on prend l'exemple de la figure 1, les différentes modalités (sain, pollué, très pollué, et hautement pollué) se recouvrent partiellement. Les zones de recouvrement sont appelées les espaces flous. Le flou ne signifie pas un manque de précision mais une diversité de perception. En effet, les modalités sont subjectives et reposent sur le présupposé de chacun. Des personnes particulièrement sensibles à la pollution auront tendance à fixer, à la baisse, la borne inférieure de chaque sous-ensemble. Si l'on prend une concentration $C_{1}$, l'entrée $C_{1}$ appartient à $60 \%$ au sous-ensemble «air sain », à $40 \%$ au sousensemble «air pollué », et à $0 \%$ aux autres sous-ensembles. En revanche, la concentration $C_{2}$ appartient intégralement au sousensemble «pollué ». Traditionnellement dans la logique floue, on évite que les modalités se recoupent plus de deux fois, cela pour des raisons de stabilité mathématique (GODJEVAC, 1999).

La relation d'appartenance entre une variable (ici la concentration de polluants) et un sous-ensemble (modalités: sain, pollué, très pollué, etc.) se dit "fonction d'appartenance». En d'autres termes, on parle de fonction d'appartenance d'une variable A à un sousensemble $B$ et il est noté: $\mu_{B}(a)$. Remarquons que la somme des fonctions d'appartenance n'est pas forcément égale à 1 , ce qui signifie notamment que les trapèzes de la figure 1 ne se coupent pas 
nécessairement à 0,5 . La fonction d'appartenance est une fonction continue. Ainsi, toute valeur de l'intervalle $[0,1]$ est atteinte, mais elle peut être atteinte en plusieurs concentrations appartenant à des modalités différentes.

L'intérêt majeur de cette méthode est la prise en compte de la perception des populations. Selon les pratiques, chacun a une idée, plus ou moins précise, de la pollution de l'air à l'échelle urbaine (cartes mentales issues de pratiques antérieures et d'informations remises à jour). Mais l'inconvénient de cette méthode est qu'elle nécessite des enquêtes de terrain pour fixer les différents seuils (concentrations et tolérance) et les degrés d'appartenance à ces différents seuils.

\section{De l'évaluation du niveau d'exposition aux polluants à la détection d'un itinéraire optimal environnemental}

La recherche d'un itinéraire optimal consiste à identifier le trajet qui minimise le temps, la distance nécessaire ou le coût économique, pour relier un point $\mathrm{A}$ à un point $\mathrm{B}$. La notion d'optimal s'appuie ici sur d'autres préoccupations, puisque la méthodologie proposée ici a pour but la détection d'itinéraires pédestres minimisant la pression environnementale subie par les piétons.

La détection d'itinéraires optimaux est une question ancienne, citons ici seulement deux exemples: le problème mathématique connu sous le nom du postier chinois qui consiste à parcourir les rues d'une ville en passant au moins une fois dans chaque rue, peu importe l'ordre de passage dans celles-ci, l'objectif étant de minimiser la distance; ou encore le problème du représentant de commerce qui effectue un choix sur les nouds (points de passage obligés) à visiter mais aussi sur l'ordre de passage aux nœuds (contraintes horaires de livraison), tout en minimisant le trajet. Ces problèmes peuvent être complexifiés en ajoutant des contraintes spécifiques (embouteillages, travaux, etc.). 
Un itinéraire pédestre est composé d'arcs (rues, boulevards, etc.), parcourus dans n'importe quel sens et dont le taux de pollution se différencie entre chaque artère en fonction de la circulation automobile, des conditions climatiques locales et de la morphologie urbaine environnante.

En ville, différents types d'itinéraires s'offrent au piéton. Plus ou moins consciemment et plus ou moins efficacement, ce dernier va sélectionner son trajet en fonction de ses usages. La personne se rendant à son travail aura tendance à privilégier le chemin le plus court en temps, tandis que le promeneur choisira davantage un chemin de bonne qualité environnementale surtout s'il coincide avec des centres d'intérêts patrimoniaux ou des espaces offerts à la vue.

En sachant que pour un itinéraire donné, le temps de trajet est inévitablement égal au temps d'exposition du piéton à une charge variable de polluants, l'un des objectifs de la démarche est de savoir s'il est préférable de prendre un chemin très court mais très pollué, plutôt qu'un chemin très long et peu pollué? Plusieurs catégories d'arcs viennent à l'esprit: arc long et pollué, arc court et sain, arc court et pollué, arc long et sain. Le premier n'est pas un optimal et il est évident que personne ne va l'emprunter, le deuxième est un optimal absolu mais n'existe que rarement dans la réalité (sinon tout le monde l'emprunterait); les deux derniers sont au centre de la discussion. Est-il préférable d'emprunter un arc court mais pollué plutôt qu'un arc long et sain? Si l'on considère seulement l'un des facteurs "distance " ou "environnement ", il est facile de départager ces deux chemins. La complexité vient du fait de devoir intégrer dans l'indicateur des facteurs qui ne sont pas du mème ordre. Mais la longueur du trajet influe fortement sur les possibilités de report sur un autre itinéraire. Il est évident que si le trajet "sain" consiste à rallonger le trajet de manière non raisonnable, le tracé optimal d'un point de vue environnemental sera délaissé au profit de la contrainte temporelle (aller au lieu de rendez-vous le plus vite possible).

La vulnérabilité du piéton est une variable qualitative fonction de l'âge, du sexe, de l'état de santé, de l'activité sportive. La concentration en polluants est estimée à partir de calculs complexes intégrant la morphologie urbaine (réseau et bâti), une base de données de trafic, une base de données d'émissions unitaires, une 
base de données climatologiques liée à un modèle de dispersion de polluants. La vulnérabilité des personnes est définie selon les classes suivantes: 1 . peu vulnérable; 2 . vulnérable; 3 . très vulnérable.

La méthode repose en partie sur la construction d'indicateurs, intégrant dans leur formulation la charge polluante de l'artère choisie mais aussi la longueur de celle-ci, ainsi que la vulnérabilité du piéton. Plus l'artère est longue, plus le fait qu'elle soit polluée est contraignant, la durée d'exposition de l'usager étant globalement proportionnelle à la longueur du trajet et inversement proportionnelle à la vitesse de déplacement. Afin de renseigner chaque arc du réseau urbain, un indicateur d'exposition a été créé. Sa formulation théorique est la suivante:

$\mathrm{E}=\mathrm{V} \cdot \mathrm{L} \cdot[\mathrm{C}]$

où $\mathrm{V}$ est la vulnérabilité du piéton, $\mathrm{L}$ la longueur de l'arc emprunté, $[C]$ la concentration en polluants de l'arc (pour un polluant spécifique).

Une fois que la valeur à affecter à chaque arc du réseau urbain est calculée, l'implémentation se fait via l'interface du logiciel ChronoVia ${ }^{4}$. Le principe de ce type d'outil est basé sur la théorie des graphes et l'algorithme du plus court chemin en distance, temps ou coût. Lobjectif est donc de minimiser la somme des contraintes. Dans le cadre d'une problématique de distance, la somme des distances pour aller d'un nœud à un autre nœud doit être minimale. C'est le même problème pour la nuisance environnementale étudiée; il s'agit de minimiser la pression environnementale subie par le piéton; autrement dit minimiser la somme des expositions, expositions qui tiennent aussi compte du temps de parcours de l'axe et de la longueur de l'axe.

ChronoVia permet une caractérisation très précise d'un réseau de voirie et de ces différents usagers, cette fonction n'a pas été utilisée dans notre exemple, puisque notre objectif n'était pas de reproduire une réalité, mais d'utiliser un cas théorique. L'usager du réseau apparaît comme un piéton qui se déplace à une vitesse constante de $5 \mathrm{~km} / \mathrm{h}$. À partir des informations disponibles dans la majorité des bases de données géographiques sur les réseaux, ChronoVia 
modélise le réseau en graphe et calcule différents indicateurs d'accessibilité et de connexité. L'outil est alors capable de détecter un itinéraire optimal entre $\mathrm{x}$ points du réseau en fonction de la distance, du temps et du coût économique, en tenant compte des facteurs de configuration du réseau (structure, sinuosité, fluidité du trafic à différentes heures, sens de circulation, feux de circulation, etc.).

Il est possible d'attribuer un coût de passage spécifique à chaque artère. Pour intégrer l'indicateur d'exposition, nous comptions utiliser la fonction de calcul d'itinéraire en fonction d'un coût économique, en ayant au préalable remplacé le coût par artère par l'indicateur d'exposition. Mais, ChronoVia a besoin d'un coût fixe qui, en fonction de la distance, ajoute une valeur représentant le coût de l'essence; cette valeur fixe qui aurait perturbé la détection des itinéraires optimaux d'un point de vue environnemental, nous a obligé à intégrer l'indicateur d'exposition à la place de la distance, seul facteur entièrement indépendant. Ce choix contraint a légèrement compliqué le protocole de calcul présenté dans la figure 2. Pour chaque itinéraire $[A B]$, nous souhaitions obtenir le tracé de l'itinéraire optimal, selon la distance et selon l'exposition environnementale aux différents niveaux de vulnérabilité, la distance parcourue et le niveau total d'exposition pour chacun des tracés.

Dans la figure 2, on parle de "calcul d'itinéraire contraint en fonction d'un tracé», cette expression mérite quelques précisions. Nous souhaitions obtenir la valeur totale d'exposition aux polluants pour les itinéraires optimaux en fonction de la distance. Or, comme nous l'avons dit précédemment, nous avons dû remplacer l'indicateur «distance» pour intégrer notre propre indicateur. Les itinéraires optimaux ont été calculés à partir de deux graphes différents, les informations disponibles sur l'un n'existant pas sur l'autre. À partir de là, la question était comment calculer la valeur de l'exposition environnementale en fonction d'un tracé optimal distance (OD) ? La détection de l'itinéraire optimal entre $A$ et $B$ peut être contrainte par le passage par un nombre quelconque de points sur le réseau. Pour résoudre notre problème, nous avons donc utilisé cette fonction en contraignant le passage à certains nœuds du réseau de manière à reproduire le tracé de l'itinéraire OD sur le graphe où nous avions intégré l'indicateur d'exposition aux polluants. 
Figure 2.

Protocole de détection d'itinéraire, de calcul des distances et des expositions totales des tracés optimaux.

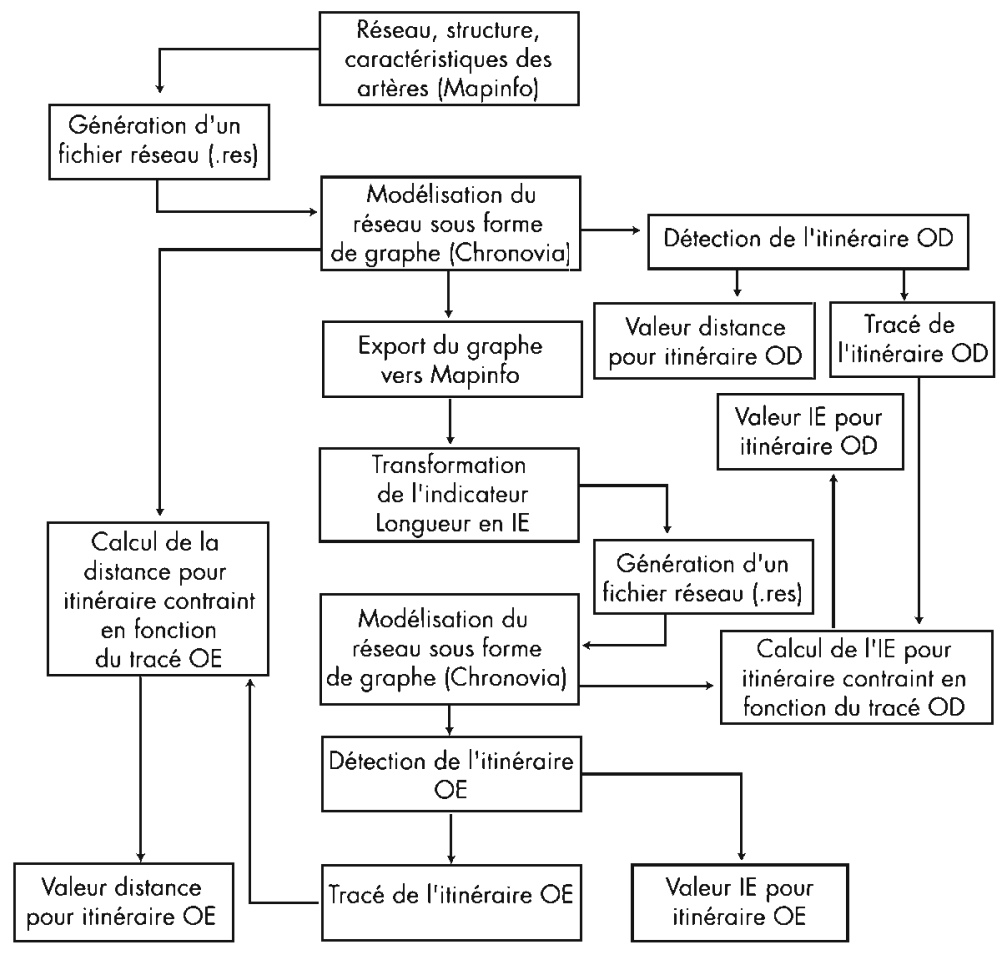

IE : indice d'exposition

$O D$ : optimum distance

$\mathrm{OE}$ : optimum environnemental

Nous obtenons alors les informations concernant la distance totale et le niveau global d'exposition pour chaque tracé d'itinéraire optimal.

\section{Applications à un espace réticulaire théorique}

Cet article, à valeur méthodologique, s'appuie sur un cas théorique de ville permettant ainsi de s'affranchir de la disponibilité de certaines données, notamment celles des flux de véhicules qui sont rarement disponibles pour l'ensemble des rues d'un quartier. 
Mais, le cas présenté est transférable à n'importe quel espace urbain sous réserve d'avoir des données de climatologie locale, de morphologie urbaine et de comptages routiers suffisamment précis. Outre l'aspect théorique des calculs et conclusions présentés, cette étude a des applications concrètes pour la société et met l'accent sur les inégalités sociales de santé comme étant un système complexe intégrant de nombreux facteurs influençant la qualité de vie. Considérons le graphe de la figure 3.

Ce graphe représente un réseau urbain constitué de 1458 arêtes, à morphologie variable (longueur, vitesse de déplacement, etc.).

Figure 3.

Typologie des artères en fonction de la quantité de $\mathrm{NO}_{2}$ et impact sur la santé.

Concentration de $\mathrm{NO}_{2}$ par artère

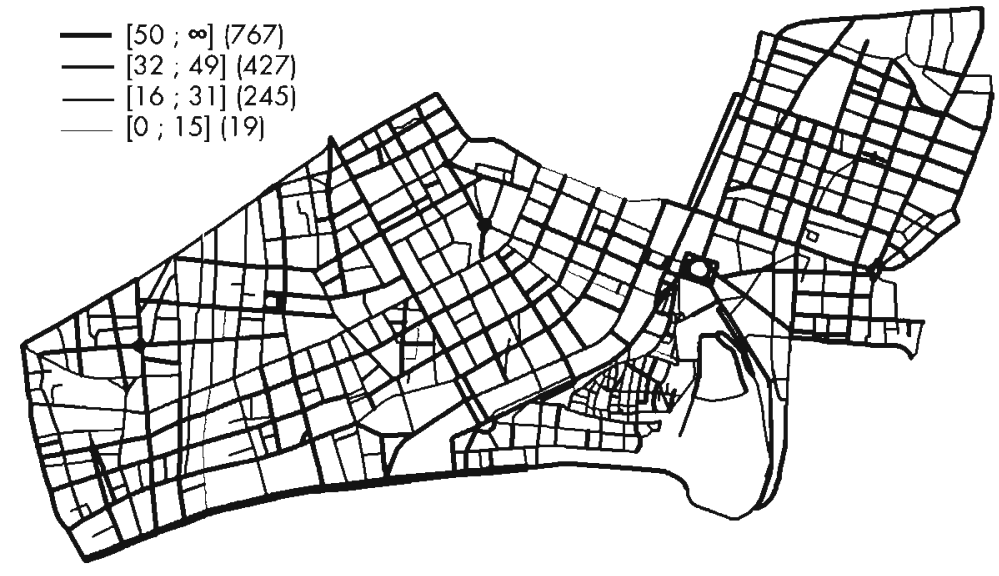

\begin{tabular}{|lll|}
\hline $\begin{array}{l}\text { Intervalle } \\
\left(\mu \mathrm{g} / \mathrm{m}^{3}\right)\end{array}$ & Qualificatif & $\begin{array}{l}\text { Effet potentiel } \\
\text { sur la santé }\end{array}$ \\
\hline$[0 ; 15]$ & Sain & $\begin{array}{l}\text { Pas d'effet sur la santé } \\
\text { chez les personnes en bonne santé }\end{array}$ \\
\hline$[16 ; 31]$ & Pollué & Présence de légère odeur \\
\hline$[32 ; 49]$ & Très pollué & Odeur \\
\hline$[50 ; \infty[$ & Hautement pollué & $\begin{array}{l}\text { Odeur et coloration brune de l'air, } \\
\text { réactions bronchiques } \\
\text { et aggravation de l'état de santé } \\
\text { des asthmatiques }\end{array}$ \\
\hline
\end{tabular}

Source: OMS, 2000 
Rappelons qu'une rue peut être découpée en plusieurs arêtes, ainsi les 1458 arêtes du graphe utilisé par ChronoVia composent 390 rues. À partir de ce réseau, de la morphologie urbaine existante, des flux de trafic et de conditions climatologiques uniformes (ce qui est raisonnable à l'échelle du quartier), nous avons estimé les charges polluantes présentes sur le réseau $[\mathrm{C}]$. La figure 3 souligne la variabilité spatiale des niveaux de pollution présents sur les artères, regroupés en quatre classes selon leurs effets sur la santé. Comme nous avons choisi trois classes

Figure $4 a$.

Itinéraire optimal entre A et B minimisant la distance $(2,978 \mathrm{~km})$.

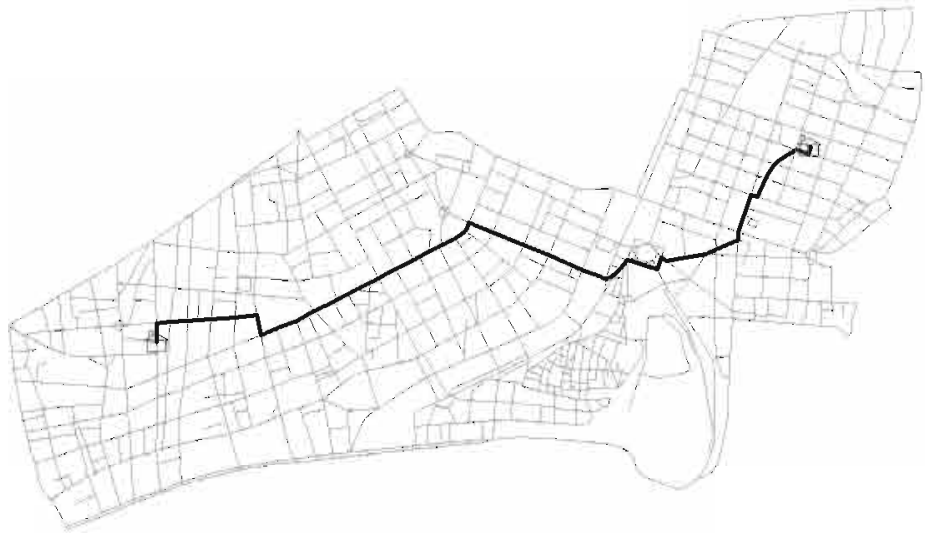

Figure $4 b$.

Itinéraire optimal entre $A$ et $B$ minimisant l'exposition pour le piéton de niveau de vulnérabilité 1 (distance $=3,223 \mathrm{~km}$; IE $=0,139$ ).

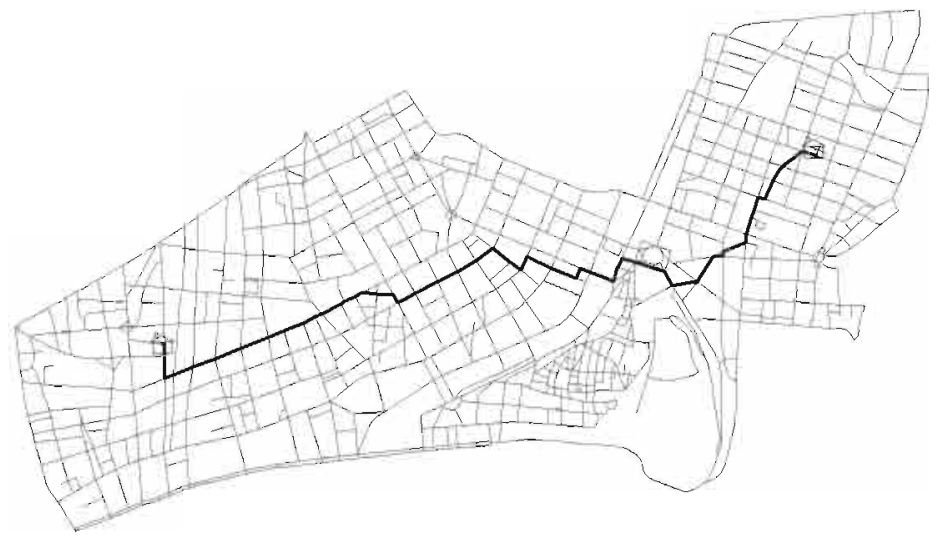


de vulnérabilité, chaque arête est affectée de trois poids différents selon ces classes et le chemin optimal correspondant est estimé dans chacun des cas. Nous avons choisi de traiter le dioxyde d'azote comme polluant car il est l'un des indicateurs de la pollution de l'air liée au trafic en milieu urbain, cette concentration est exprimée en $\mu \mathrm{g} / \mathrm{m}^{3}$. En effet, les voitures sont à l'origine de $32 \%$ des émissions de dioxyde d'azote dans l'air. La valeur guide pour ce polluant est de $40 \mu \mathrm{g} / \mathrm{m}^{3}$ en moyenne annuelle. Comme les données de circulation automobile sont aussi en

Figure 4c.

Itinéraire optimal entre $A$ et $B$ minimisant l'exposition pour le piéton de niveau de vulnérabilité 2 (distance $=3,617 \mathrm{~km}$; IE $=0,263$ ).

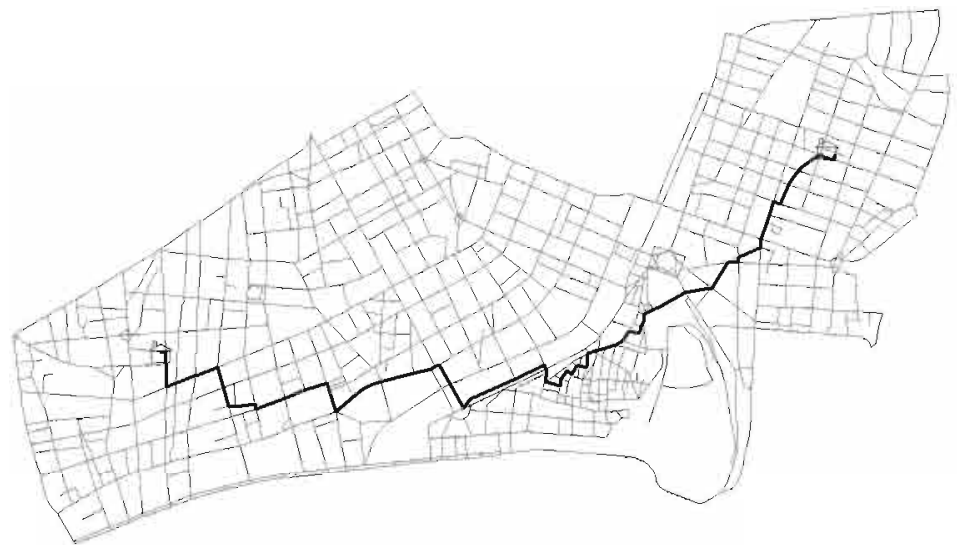

Figure 4d.

Itinéraire optimal entre $A$ et $B$ minimisant l'exposition pour le piéton de niveau de vulnérabilité 3 (distance $=3,511 \mathrm{~km}$; IE $=0,4$ ).

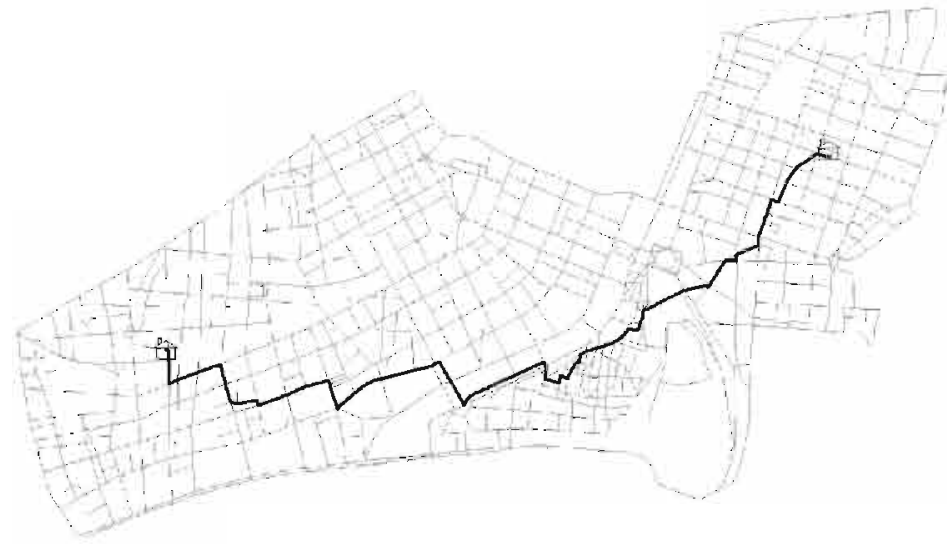


Figure $5 \mathrm{a}$.

Itinéraire optimal entre A, B, C minimisant la distance $(3,283 \mathrm{~km})$.

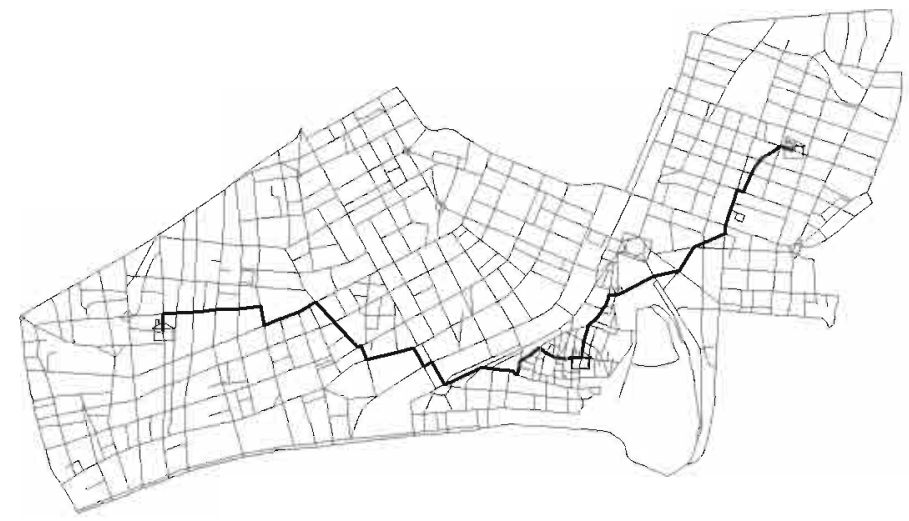

Figure $5 b$.

Itinéraire optimal entre A, B, C minimisant l'exposition pour le piéton de niveau de vulnérabilité 1 (distance $=3,484 \mathrm{~km}$; IE $=0,139$ ).

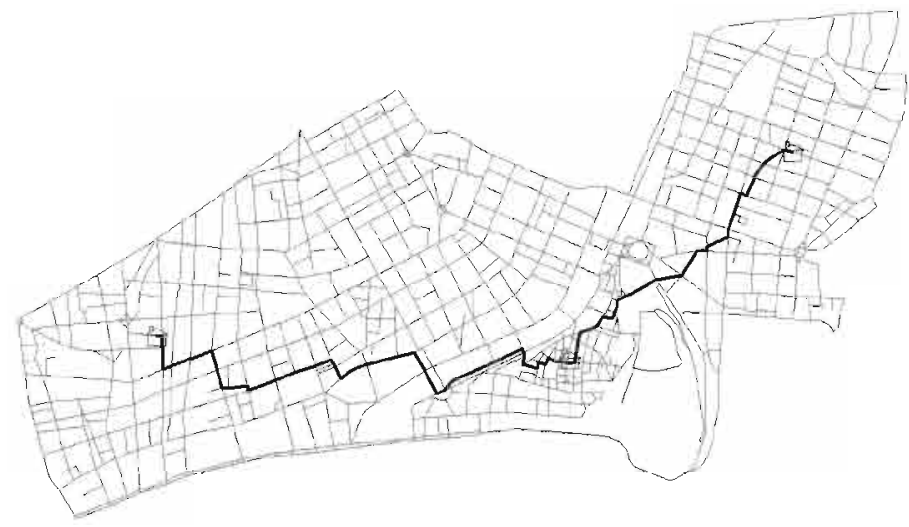

moyennes annuelles, les expositions potentielles sont elles aussi des expositions moyennes annuelles. Pour obtenir des informations en temps réel et une réactualisation en continu des itinéraires optimaux, il faudrait que l'ensemble des données puisse être disponible au pas de temps horaire.

Si on considère d'abord les itinéraires en deux points $\mathrm{A}$ et $\mathrm{B}$, on remarque que le tracé des optimaux environnementaux diffère beaucoup entre le niveau de vulnérabilité 1 et les deux autres niveaux de vulnérabilité. En effet, si l'itinéraire Optimum environnemental 
Figure $5 c$.

Itinéraire optimal entre A, B, C minimisant l'exposition pour le piéton de niveau de vulnérabilité 2 (distance $=3,714 \mathrm{~km}$; IE $=0,27$ ).

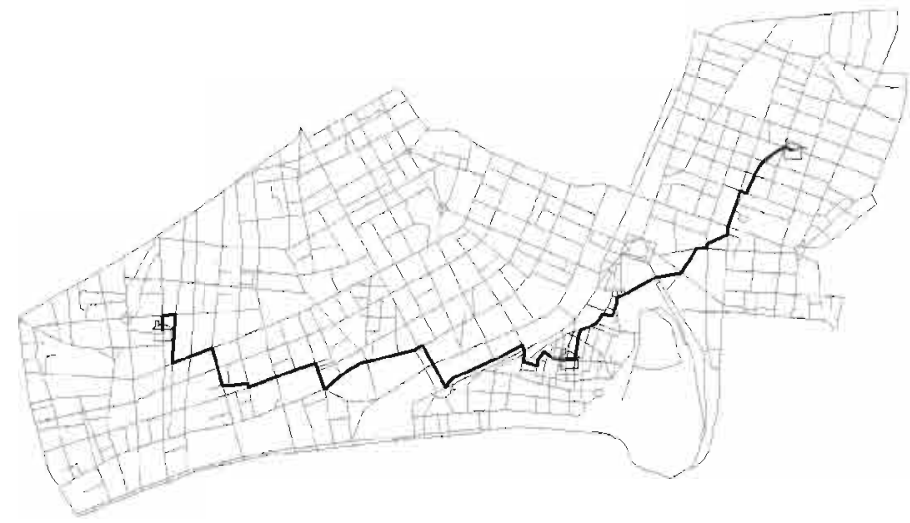

Figure 5d.

Itinéraire optimal entre A, B, C minimisant l'exposition pour le piéton de niveau de vulnérabilité 3 (distance $=3,581 \mathrm{~km}$; IE $=0,404$ ).

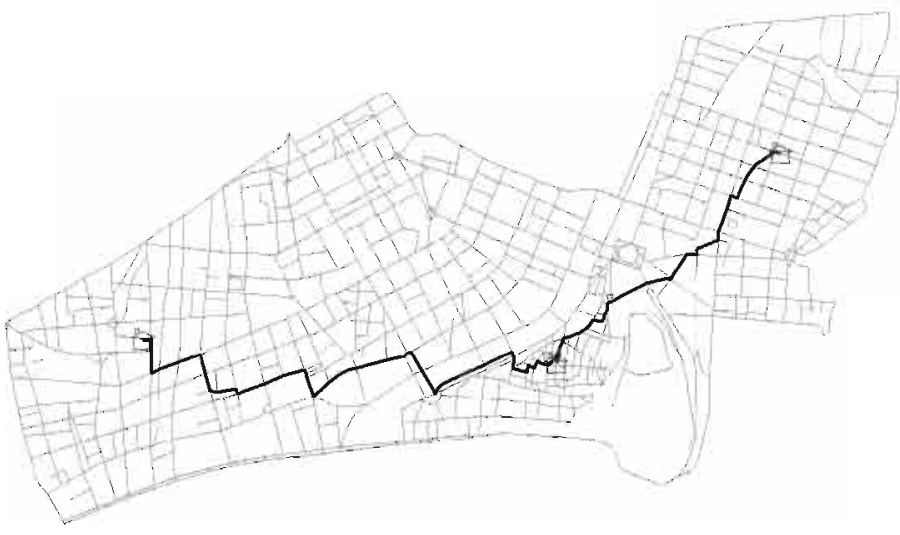

vulnérabilité $1(\mathrm{OEVl})$ prend une trajectoire relativement proche de celle de l'Optimum distance (OD); la trajectoire de OEV2 et OEV3 est quasiment inverse à celle de l'OEV1. En fonction du niveau de vulnérabilité du piéton, le tracé de l'itinéraire optimal est donc susceptible de varier fortement. En effet, l'indicateur est conçu pour donner un rôle prépondérant au degré de sensibilité de l'usager du réseau; un individu en pleine santé (V1) étant moins affecté par la nuisance, pour minimiser «le coût écologique», va avoir tendance à minimiser avant tout sa distance de parcours. 
Par contre, pour les populations plus vulnérables (V2, V3), étant donné la pondération du facteur d'exposition, l'élément « distance» perd de son importance. Pour minimiser l'exposition, il devient alors "écologiquement rentable» d'emprunter un chemin plus long (distance OEVl $=3,223 \mathrm{~km} ; \mathrm{OEV} 2=3,617 \mathrm{~km}$ ) afin d'éviter les artères du réseau de voirie dont la qualité environnementale est trop dégradée. Si on observe les tracés des itinéraires des figures 4 a à $5 \mathrm{~d}$, on a vraiment l'impression que par comparaison à l'OD ou l'OEVl qui ont des tracés assez rectilignes, les OEV2 et V3 modélisent une stratégie d'évitement d'une partie du réseau, quitte à devoir rallonger nettement le parcours.

Pour les itinéraires en trois points $\mathrm{A}, \mathrm{B}, \mathrm{C}$, nous avons simplement ajouté un point de contrainte à partir des points $\mathrm{A}$ et $\mathrm{B}$, ce qui avait pour effet de limiter les variations des tracés. Avec cette contrainte de passage qui pourrait correspondre à un site culturel ou d'intérêt patrimonial, les OE des trois niveaux de vulnérabilité ont des tracés quasi identiques et varient seulement au niveau de l'indice d'exposition. Les points de départ et de contrainte que nous avons sélectionnés aléatoirement aboutissent à un cas particulier. En effet, la contrainte de passage correspond à l'itinéraire $[A B]$ de l'OE2 et l'OE3 et donc à une certaine distance de l'OD et l'OEl. Cela explique pourquoi les valeurs globales des indices d'exposition varient peu suite à l'ajout d'une contrainte de passage supplémentaire. Par contre, pour l'OEl, même si la valeur de l'indice d'exposition reste stable, la trajectoire se modifie clairement. Enfin, quelles que soient les contraintes extérieures imposées (lieux de passage ou pas), plus la vulnérabilité augmente, plus le niveau total d'exposition augmente.

Pour approfondir la comparaison entre les itinéraires obtenus en minimisant la distance et ceux obtenus en minimisant la contrainte environnementale, on peut envisager la création de nouveaux indicateurs.

Le premier consisterait à évaluer le nombre d'arcs qui diffèrent (ou qui sont communs) entre le parcours optimal d'un point de vue distance et le parcours optimal d'un point de vue environnemental. Cet indicateur, simple à calculer, permet déjà de souligner des différenciations spatiales importantes dans les itinéraires. Remarquons que le parcours optimal d'un point de vue environnemental est le 
même pour toutes les classes de vulnérabilité, seul le niveau d'exposition total va varier. Le nombre d'arcs différents est un très bon indicateur du nombre de contournements nécessaires pour éviter des artères dont l'exposition potentielle est supérieure. Toutefois, cet indicateur ne tient pas du tout compte de la morphologie des tracés; c'est-à-dire que deux itinéraires parallèles et proches mais n'ayant aucune artère commune seront considérés comme plus différents que deux itinéraires qui n'auraient qu'une artère en commun à partir de laquelle les trajectoires d'itinéraires divergeraient complètement.

Le deuxième indicateur que nous définissons est le rapport $\left[\left(\mathrm{L}_{\mathrm{OE}}-\mathrm{L}_{\mathrm{OD}}\right) / \mathrm{L}_{\mathrm{OD}}{ }^{*} 100\right.$ (tabl. 2). Dans cette formule le terme $\mathrm{L}_{\mathrm{OE}}$ désigne la longueur de l'itinéraire optimal d'un point de vue environnemental, tandis que $\mathrm{L}_{\mathrm{OD}}$ désigne la longueur de l'itinéraire optimal d'un point de vue distance. Cet indicateur donne le pourcentage d'augmentation de la longueur du trajet relatif à la longueur du trajet de l'optimum distance. Il permet ainsi de voir si l'augmentation de la longueur de l'itinéraire est raisonnable face au respect de la contrainte environnementale.

Tableau 2.

Résultats pour l'ensemble des indicateurs

\begin{tabular}{|c|c|c|}
\hline Itinéraire optimal [AB] & Distance (km) & Exposition totale \\
\hline Distance & 2,978 & \\
\hline OE Vulnérabilité 1 & 3,223 & 0,139 \\
\hline OE Vulnérabilité 2 & 3,617 & 0,263 \\
\hline OE Vulnérabilité 3 & 3,511 & 0,4 \\
\hline Itinéraire optimal $[\mathrm{ABC}]$ & Distance $(\mathrm{km})$ & Exposition totale \\
\hline Distance & 3,283 & \\
\hline OE Vulnérabilité 1 & 3,484 & 0,139 \\
\hline OE Vulnérabilité 2 & 3,714 & 0,27 \\
\hline Oe Vulnérabilité 3 & 3,581 & 0,403 \\
\hline Indicateur: $\left[\left(\mathrm{L}_{\mathrm{oe}}-\mathrm{L}_{\mathrm{od}}\right) /\right.$ & -od $]$ * 100ltinéraire $[A B]$ & Itinéraire $[A B C]$ \\
\hline OE Vulnérabilité 1 & 8,227 & 6,122 \\
\hline OE Vulnérabilité 2 & 21,457 & 13,128 \\
\hline OE Vulnérabilité 3 & 17,898 & 9,077 \\
\hline
\end{tabular}




\section{Conclusion}

Les problèmes d'itinéraires cherchent systématiquement à optimiser certains paramètres comme le temps ou la distance. Nous avons vu qu'il est possible d'introduire de nouvelles préoccupations dans le choix de ces itinéraires, par exemple des questions environnementales (qualité de l'air sur les axes ou encore nuisances sonores). L'intérêt majeur de la méthode proposée réside dans le couplage vulnérabilité des personnes - vulnérabilité des axes urbains face à la charge polluante.

Dans l'objectif d'une requalification des centres urbains, notamment par la mise en place de nouvelles aires piétonnes, ou de voies de circulations réduites, la qualité de vie de ces espaces tend à s'améliorer, la notion de développement durable sous-tendant cette évolution. Ainsi, le piéton commencerait à retrouver une place en ville. Par le biais de rétroactions, cela va améliorer la qualité de vie globale de nos villes. En effet, le piéton constatant une amélioration de la qualité environnementale de certains itinéraires empruntés aura tendance à favoriser les modes de déplacements doux et contribuera ainsi à enrichir la qualité de vie, phénomène semblable à l'entropie d'un système fermé. Cependant, en termes d'aménagements urbains, on ne peut rendre totalement la rue au piéton et des axes structurants de la ville devront être dédiés principalement à la mobilité automobile. Dans cette optique, le problème d'itinéraires optimaux environnementaux prend un tout nouveau tournant: comment est-il possible d'éviter les axes structurants sachant que ceux-ci sont pénalisants d'un point de vue santé publique?

Compte tenu du rythme soutenu de diffusion des technologies liées au GPS (désormais disponibles sur les GSM), il est fort probable qu'à court ou moyen terme de nouvelles générations de GPS voient le jour. En s'adaptant aux nouvelles pratiques de mobilité en ville, et en tenant compte des préoccupations des populations, ces nouveaux GPS pourraient bien intégrer de nouveaux facteurs d'optimisation, entre autres environnementaux. 


\section{Références bibliographiques}

Borteux M., 2001 - Transports: Choix des investissements et coûts des nuisances. Commissariat Général du Plan, 323 p.

BOUCHON-MEUniER B., 1993 - La logique floue. Presses Universitaires de France, coll. Que sais-je? $128 \mathrm{p}$.

CHARRE J., 2003 - "L'objet géographique, chose disciplinaire ». In Maby J. (dir.): Réflexion sur la nature des objets et des indicateurs de la géographie: 308-316.

GODJEVAC J., 1999 - Idées nettes sur la logique floue. Lausanne, Presses Polytechniques et Universitaires Romandes, coll. Informatique, $125 \mathrm{p}$.

Maignant G., 2002 - Pollution et développement durable des villes françaises, êtude de cas: Nice, Marseille, Lyon et Paris. Thèse de géographie de l'université de Nice-Sophia Antipolis, 700 p.

MCMiChaEl T., 2001 - La santé en milieu urbain: conséquences de la mondialisation dans les pays en voie de développement. Bulletins de l'OMS, $8 \mathrm{p}$.

MORIN E., 2002 - «À propos de la complexité ». Discours d'entrée CNRS, 14 janvier.

Nicolescu B., DE Freitas L., MORIN E., 1994 - « Charte de la transdisciplinarité». Adoptée lors du Premier congrès mondial de transdisciplinarité, Convento da Arrábida, Portugal, 2-7 novembre.

OMS., 2000 - Air Quality Guidelines for Europe. Second edition, WHO Regional Publications, European Series, n91, 273 p.

Pini P., LAVAdinho S., 2005 - «Développement durable, mobilité douce et santé en milieu urbain, actes du colloque». In: Développement urbain durable, gestion des ressources et gouvernance, Lausanne, 8 p.

ROQUEPLO P., 1987 - «Les pluies acides considérées comme un accident au ralenti. In Fabiani J.-L., Theys J. (dir.) : La Société vulnérable, Paris, Presses de l'École normale supérieure, $674 \mathrm{p}$.

ZADEH L., 1965 - Fuzzy sets. Information control, 8: 338-353. 

Partie 3

Comportements

humains et maladies

liés à l'environnement dans les pays

du Sud 



\section{L'étude des risques sanitaires liés à l'eau dans l'environnement urbain: I'exemple de la ville de Chennai, Inde du Sud}

\section{Bernard MONDET, Thomas SEYLER}

Gérard SALEM, Jean-Paul GONZALEZ

Les modifications de l'environnement, souvent dues aux activités humaines, ont été généralement associées aux phénomènes d'émergence ou de ré-émergence de maladies infectieuses. Les exemples sont nombreux et le plus souvent liés aux migrations, aux échanges et (ou) regroupements de populations, et à la modification de l'environnement que cela implique. En Afrique de l'Ouest, la construction de barrages a précédé l'extension géographique de l'onchocercose et, plus récemment sans doute, celle de la fièvre de la vallée du Rift (SALuzzo et al., 1987). La légionellose, maladie bactérienne, est en relation directe avec l'environnement urbain humide (systèmes de climatisation), la contamination de l'homme s'effectuant par inhalation. En Europe et aux États-Unis, la ré-émergence de Mycobacterium tuberculosis, sous forme de souches multi-résistantes, est associée, entre autres facteurs, à la dégradation des conditions de vie dans les agglomérations des populations les plus vulnérables. La part de la population humaine globale vivant dans les villes ne cesse d'augmenter et, selon «UN-Habitat», elle est passée du 
tiers de la population de la planète en 1950 à plus de la moitié actuellement, et devrait atteindre, vers 2050, les deux tiers de la population mondiale ( 6 milliards sur les 9 estimés). L'urbanisation rapide entraîne des changements environnementaux et sociaux profonds dans l'ensemble des écosystèmes urbains et périurbains ainsi qu'à leur interface et les métropoles tropicales sont celles qui subissent les plus intenses et les plus rapides modifications et dégradations environnementales (MAITI et AGRAWAL, 2005).

Un des plus grands défis, face à ces bouleversements écologiques, est celui de la gestion de l'eau: accès à l'eau potable, évacuation des eaux de pluies et des eaux usées ce qui, quand les systèmes sont déficients, a une influence directe sur la santé des hommes et des animaux. Les maladies peuvent être directement liées à l'eau ou être transmises par des vecteurs qui peuvent passer par une phase aquatique (les larves de moustiques), avoir une sensibilité extrême à l'humidité (les tiques) ou encore posséder des niches écologiques dépendantes de la nappe phréatique (les rongeurs). Dans ces conditions, l'analyse globale des problèmes de santé dans les grandes villes passe par l'étude des conditions d'émergence et d'évolution des maladies liées à l'eau, et possède trois orientations majeures:

- l'exposition dans le temps et dans l'espace des populations humaines aux différents facteurs de risque, naturels et anthropiques; - la vulnérabilité des populations aux maladies émergentes et (ou) inédites;

- la capacité des vecteurs de s'adapter à leur environnement et celle des hôtes de s'adapter au ( $\mathrm{x})$ risque(s).

Cette étude ne peut donc s'envisager que par une approche intégrée, interdisciplinaire et systémique ayant pour objectif ultime le développement d'un système d'alerte précoce. Pour mieux appréhender la structure complexe d'une telle étude, nous nous basons sur un exemple concret, celui de la ville de Chennai, anciennement Madras, capitale de l'État du Tamil Nadu en Inde du Sud. 


\section{Chennai, mégapole du Sud}

Si le taux de croissance décennal a baissé de $17,24 \%$ (période 1981-1991) à 9,76\% (période 1991-2001), la densité humaine a augmenté, elle, de 22077 à 24231 habitants $/ \mathrm{km}^{2}$ (Census of India, 2004). Actuellement, la ville de Chennai intra-muros abriterait 4,3 millions d'habitants sur $172 \mathrm{~km}^{2}$ et le «Grand Chennai " aurait 7 millions d'habitants inégalement répartis sur $1166 \mathrm{~km}^{2}$. Dans cette ville, à l'urbanisation incessante, aux changements environnementaux majeurs, les disparités socioéconomiques sont très grandes. Le pourcentage de population des bidonvilles (anglais: slums) est passé de 13,8\% en 1981, à $17,7 \%$ en 2001 (Maiti et AGRAWAL, 2005), pour être estimé actuellement à 26\% (Chandramouli, 2003). De la périphérie au cœur historique de la vieille ville, de nombreux écosystèmes ruraux, périurbains et urbains se côtoient, se mélangent, parfois de façon complexe, souvent en relation étroite avec l'environnement physique et aussi humain. Dans ce contexte de mosaique éco-sociologique, les maladies liées à l'eau représentent une très grande part des pathologies existantes. Ce sont des maladies comme les fièvres diarrhéiques, la leptospirose et le choléra d'une part, et celles à insectes vecteurs comme le paludisme, les fièvres à virus dengue et à virus chikungunya d'autre part.

Pour une étude de ce type sur le risque d'émergence de pathologies liées à l'eau, dans un contexte d'urbanisation et de changements environnementaux, un état des lieux doit être un préalable à toute tentative d'analyse et doit porter sur les hommes, les maladies et leurs vecteurs, ainsi que sur l'influence du climat sur les conditions d'apparition et de développement des maladies dans la ville (PAPPACHAN et al., 2004a).

\section{Méthodologie}

Dans le cas de la ville de Chennai, c'est d'une hypothèse simple que va débuter notre réflexion: la vulnérabilité et la capacité d'adaptation (ou de réaction) des diverses populations humaines 
face aux risques sanitaires liés à l'eau présentent des différences intra-urbaines car, comme l'affirment V. MOHAN et al. (2001), le statut socio-économique d'un individu a une influence sur sa santé et celle de sa famille ou de son entourage.

Le fil conducteur est donc l'étude des hommes dans leurs environnements par une approche intégrée et interdisciplinaire. Comme à Salvador de Bahia, au Brésil (Teixeira et al., 2002a), l'épidémiologie dans des agglomérations complexes peut être effectuée après un travail de sélection de zones représentatives des différents écosystèmes et espaces sociaux urbains. Les données fondamentales de sociologie, d'économie, de biologie et d'éthologie seront alors collectées dans ces zones sentinelles. L'entomologie a une place importante dans l'étude des maladies transmissibles qui doit se baser sur une bonne connaissance de la répartition des gîtes larvaires des moustiques, des densités des stades larvaires et des adultes, des zones de contacts entre l'homme et le vecteur. Chez ces derniers, la présence de virus peut, d'ailleurs, être systématiquement recherchée pour estimer la répartition spatio-temporelle des risques de transmission virale (CRME, 2003) tout comme la présence de Plasmodium peut renseigner sur celle du paludisme (TRAPE et al., 1992). Les zones de répartition de ces maladies varient en fonction des capacités de déplacement, actif et passif, des vecteurs infectés. En sciences sociales, le cadre des études est constitué autour des notions d'analyse de vulnérabilité (BOHLE, 2001; SAUERBorn et al., 1996), largement développées en géographie de la santé comme à Pikine, au Sénégal, par exemple (SAlem, 1998).

Ces premières études portent donc sur la vulnérabilité, l'exposition et l'adaptation des populations humaines à leur environnement et aux pathologies circulantes. Celles sur la vulnérabilité ont pour base une cartographie dans le temps et dans l'espace des infections liées à l'eau. L'exposition est mise en évidence par la description dynamique de l'environnement urbain et périurbain, à la fois physique (saisons, microclimats, infrastructures, habitats, collections d'eau), biologique (gîtes larvaires des vecteurs, dynamique des populations de rongeurs, répartition des agents pathogènes) et social (niveau des revenus, appartenance à une caste, mobilité, accès aux soins de santé). Les données obtenues permettront de 
repérer, pour chacune de ces catégories, des facteurs déterminant l'inégalité face au risque d'infection. Enfin, les populations humaines urbaines possèdent des comportements d'adaptation variables qui dépendent de multiples facteurs qu'il faudrait caractériser. Il s'agit donc d'étudier et d'analyser la perception du risque (KUMAR et al., 2003) en relation avec les maladies existantes et celles émergentes, puis de rechercher les types de réactions à court et moyen termes les mieux adaptés au contexte social.

L'ensemble de ces données physiques, biologiques et sociales pourrait trouver sa place dans un système d'information géographique (BECK et al., 2000) malgré certaines difficultés (MOLONEY et al., 1998).

\section{Matériel et méthodes}

Le développement de bases de données spatialisées sur l'environnement et la santé dans la métropole de Chennai s'impose, ce qui devrait permettre de cartographier dans le temps et dans l'espace l'incidence des maladies liées à l'eau, d'obtenir une image dynamique en zones périurbaines et urbaines des environnements physique, biologique et social, de mettre en évidence les comportements et l'adaptabilité des populations les plus vulnérables au risque d'émergence au sens large. Vu l'hétérogénéité des zones d'étude, une attention particulière doit être donnée à la dimension spatiale grâce à des données géoréférencées par GPS (Global Positioning System) et traitées à l'aide du logiciel ArcGIS@. La classification des images satellitaires peut utiliser Erdas Imagine(C), les résultats des questionnaires et des enquêtes pouvant être intégrés et analysés avec Epilnfo@. L'ensemble des données pourrait être utilement traité dans un logiciel étendu d'information, SavaneSIG(C) (www.savgis.org). Des questionnaires individuels et des entretiens sont à mener au cours de réunions de quartier pour définir des zones sentinelles représentatives avec, en particulier, consultation de groupes informels de femmes, de personnel social, enseignants, infirmiers, chefs de communauté, etc. 
Des outils de diagnostic relativement courants peuvent être utilisés pour les différentes maladies: bandelettes de diagnostic rapide fournies par les instituts Pasteur de Paris et de Madagascar pour les diarrhées et le choléra; tests lgM et IgG pour la détection des anticorps dans la recherche des leptospiroses et des arboviroses, avec confirmation au besoin par ELISA. La technique RT-PCR (CHUNG et PANG, 2002) peut être adoptée pour la détermination du sérotype et, en cas d'épidémie, l'ARN viral utilisé pour identifier l'origine des souches circulantes à partir d'échantillons de sang fournis par les services de santé des différents hôpitaux.

En entomologie, les enquêtes visent à la recherche de gîtes larvaires et à la capture de stades larvaires et adultes afin de déterminer les index entomologiques (STRICKMAN et KITTAYAPONG, 2002; FOCKS et al., 2000). Divers types de pièges peuvent être utilisés, en particulier le nouveau piège à moustiques BG-Sentinel ${ }^{\circledR}$ qui apparaît actuellement le plus efficace des pièges à odeur pour capturer des Aedes (KROCKEL et al., 2006). Des dissections de femelles adultes peuvent compléter les données en précisant l'abondance des Plasmodium du paludisme ou la variation, au cours du temps, du taux de parité et de l'âge physiologique des adultes, donc des taux de survie (MONDET, 1996).

\section{Les objets d'une recherche transdisciplinaire}

\section{Les hôtes et leurs environnements}

Létude des facteurs d'émergence et de persistance de maladies au sein de populations humaines ne peut pas se limiter à une analyse strictement biologique des agents infectieux et des couples vecteurs/réservoirs. Il s'agit également de cerner les comportements humains et les modifications environnementales qui peuvent entraîner et/ou perpétuer certains risques sanitaires. De nombreux travaux ont montré l'impact sanitaire qu'ont pu avoir les modifications des modes de vie de communautés humaines soumises aux évolutions d'un environnement changeant. Certains auteurs situent l'émergence accrue des maladies infectieuses chez l'homme 
à l'époque de la sédentarisation qui conduisit à l'accroissement des groupements humains et à la domestication d'animaux. Des études ont établi le lien entre diffusion de certaines maladies comme la bilharziose, l'onchocercose ou l'encéphalite japonaise et la construction d'infrastructures hydrauliques. En Inde, par exemple, dans l'État du Karnataka, c'est la déforestation et l'expansion de terres cultivables qui ont exposé les populations locales au vecteur du virus de la fièvre hémorragique de la forêt de Kyasanur (WORK et TRAPIDO, 1957).

Les grandes cités en Inde ont vu une urbanisation rapide en raison de la poussée économique et démographique. Avec une pression croissante de la population dans un environnement urbain dès le départ très médiocre, les conditions de vie dans les agglomérations et en zones périurbaines ne peuvent que se détériorer avec le temps (voir les différents rapports annuels de la Banque mondiale, www.worldbank.org/data). Les modifications des comportements humains, ainsi que la transformation de nature anthropique du milieu de vie, sont particulièrement présentes dans les grandes villes du Sud comme la métropole de Chennai, représentative pour plusieurs raisons: un territoire densément peuplé, un espace hétérogène par ségrégation résidentielle spatiale (DupONT, 2004), par différents types d'habitat (BUNCH, 1997), par niveau d'infrastructure, par offre et accès aux soins de santé. C'est aussi un espace ouvert où la mobilité humaine est grande, aussi bien à l'intérieur de la ville qu'avec les villages alentour, la région et le reste du monde. Ces trois caractéristiques (densité, hétérogénéité et ouverture) doivent être prises en considération au moment du montage des enquêtes de santé (SALEM, 1998; TEIXEIRA et al., 2002 b). Enfin, c'est un espace où la surveillance épidémiologique reste limitée à certaines pathologies (DEEPA et al., 2003), alors que des événements sanitaires et des études ponctuelles suggèrent des conditions propices à l'émergence brutale de pathologies, comme le choléra 0139 en 1992 (DHAMODARAN et al., 1995) ou de fièvres à virus chikungunya.

Étroitement liée au processus d'urbanisation, la difficile gestion de l'eau dans la métropole de Chennai pose des problèmes majeurs de santé publique et de société: pénurie d'eau potable, pollution des rivières et canaux, inondations en période de 
mousson, qui sont autant de situations critiques créées en grande partie par l'homme et qui favorisent l'émergence et la persistance de pathologies liées à l'eau. La gestion de l'eau au sein de la métropole est aussi un enjeu politique de première importance, suscitant de nombreux débats (Citizens' water forum, 2006) et un problème journalier pour la majorité des habitants. Le stockage de l'eau à l'intérieur des habitations, parfois indispensable, favorise le développement des populations de moustiques, essentiellement Aedes aegypti et, de fait, les risques d'apparition de la fièvre dengue (RODHAIN, 1983). Ce processus d'urbanisation à Chennai n'est pas un processus spatial homogène, certains quartiers n'étant pas reliés au réseau de distribution d'eau, d'autres espaces urbains n'ayant pas de système d'évacuation des eaux. Il en découle ainsi des disparités de santé étroitement liées à une ségrégation spatiale (CHANDRAMOULI, 2003). Ces différences intra-urbaines sont très importantes à étudier du point de vue épidémiologique comme sociologique (REITER et al., 2003).

\section{Les maladies liées à l'eau et leurs agents infectieux}

Les maladies liées à l'eau sont les maladies transmises à l'homme dont l'existence est directement ou indirectement liée à un environnement aquatique. Elles peuvent être en relation directe avec l'eau souillée dans laquelle se développe un agent infectieux (diarrhées dues à des gastro-entérites virales ou bactériennes, choléra, etc.). Elles peuvent être aussi indirectement liées à un environnement d'eaux usées et à la présence d'hôtes/vecteurs porteurs d'un germe pathogène, comme c'est le cas pour la leptospirose hémorragique dont l'agent infecte les rongeurs domestiques ou péridomestiques qui vont, eux-mêmes, contaminer les eaux de consommation par leurs déjections (Afsaa, 2006; WHO, 2003; Le choléra, 2006). Enfin, les maladies infectieuses associées à l'eau peuvent être caractérisées par leur environnement aquatique et mises en relation avec différents types de collections d'eau plus ou moins saines. Les phases aquatiques (état larvaire) des différentes espèces d'arthropodes vecteurs de maladies (tels les moustiques vecteurs de filaires, de plasmodies ou d'arbovirus) possèdent des exigences, essentiellement d'ordre physiologique, qui entraînent le choix de tel ou tel milieu aquatique. 
À Chennai, des études doivent porter sur la leptospirose (MUTHUSETHUPATHI et al., 1995), le choléra (DHAMODARAN et al., 1995) et le paludisme urbain qui est reconnu depuis longtemps en Inde. Transmis par un anophèle (Anopheles stephensi) utilisant des puits et des citernes comme gites larvaires, le paludisme est endémique à Chennai, en raison de l'apport permanent de nouveaux réservoirs de Plasmodium que sont les populations humaines venant de zones endémiques, émigrant en ville (Annual Action Plan, 2003).

Parmi les arboviroses, la fièvre dengue et la fièvre due au virus chikungunya, transmises par Aedes aegypti et (ou) Aedes albopictus dominent la scène. En Asie, les virus co-circulent souvent au cours d'épisodes épidémiques comme à Nagpur, en Inde, par exemple, en 1965 (RODRIGUes et al., 1972) ou à Chennai (MYers et al., 1965). Depuis le milieu des années 1950, plus de 60 épidémies de dengue ont été rapportées en Inde dans tous les États indiens (TeWARI et al., 2004) avec une circulation virale possible dans différents écosystèmes du monde rural et du monde urbain (CECILIA, 2004). Une épidémie majeure de fièvre dengue et de fièvre dengue hémorragique a touché la capitale Delhi et les villes alentour en 1996-1997, puis en 2006-2007. Au cours de la première phase de cette épidémie, 423 décès ont été reportés et 10252 cas hospitalisés (BROOR et al., 1997). Dans la ville de Delhi, on a confirmé 1978 cas en 2006, puis 505 en 2007 (The Hindu, 5 janvier 2008). À Chennai, la transmission s'effectue essentiellement au cours de la mousson (située, dans cette région de l'Inde, entre les mois d'août/septembre et décembre/janvier).

En Inde, des épidémies de fièvre virale à chikungunya ont été observées entre 1963 et 1965 à Kolkota et le long de la côte est jusqu'à Chennai et Pondicherry, puis dans le centre du pays entre 1965 et 2000 (MOURYA et al., 2004). La dernière épidémie reconnue à Chennai remonte à 1965, mais entre décembre 2005 et avril 2006, plus de 31000 cas se déclaraient dans l'État voisin d'Andhra Pradesh dont $10 \%$ étaient des infections mixtes dengue chikungunya selon le National Institute of Virology de Pune, Inde (MISHRA, 2004) et comme précédemment observé (RODRIGUES et al., 1972). L'épidémie s'est poursuivie, a pris de l'ampleur et, entre décembre 2005 et la mi-octobre 2006, on estimait à plus de 1350000 le nombre de cas dans tous les États de la moitié sud du pays (The Hindu, 18 octobre 2006). Il n'existe pas de vaccin ou de traitement 
spécifique de ces affections qui peuvent engendrer des syndromes cliniques graves (hémorragies, encéphalites, état de choc) en particulier chez l'enfant ou l'adulte jeune (BANERJEE, 1996; VIJAYAKUMAR et al., 2005).

\section{Les vecteurs}

Les vecteurs des virus de la dengue et du chikungunya (DENV et CHIKV), Aedes aegypti et Aedes albopictus, se rencontrent dans les milieux urbains et ruraux. Leurs stades larvaires sont aquatiques et donc dépendants de l'existence de gîtes contenant de l'eau, qui peuvent être d'origine anthropique (objets abandonnés tels boittes de conserves, pots, citernes, pneus, etc.) ou naturelle (trous d'arbres en particulier). Le rôle dans la transmission de nombreux virus, in natura, par Ae. agypti est connu et prouvé depuis longtemps et Ae. albopictus s'est avéré responsable de l'épisode épidémique dévastateur qui a touché plus du tiers de la population humaine de l'île de la Réunion en 2005-2006 et entraîné la mort de plus de 170 personnes (DuHamel et al., 2006). Ae. albopictus est un vecteur efficace d'arbovirus et peut être porteur du virus CHIK dans la nature (HAWLEY, 1988). On le soupçonne également d'avoir joué un rôle dans certaines des épidémies de dengue en Inde (Mourya et al., 2004).

Le principal vecteur du paludisme urbain, maladie endémique (donc non émergente) dans les villes indiennes, est Anopheles stephensi dont les gites larvaires favoris sont les réservoirs d'eau douce placés sur les toits des maisons (Annual Action Plan, 2003). La bio-écologie de cette espèce est parfaitement connue, en particulier dans un environnement urbain en changement constant, comme à Delhi (BATra et al., 2001). A Chennai, les cas de paludisme se rencontrent plus particulièrement dans le quartier de George Town, où se concentrent les travailleurs récemment immigrés de villages où le paludisme se maintient à l'état endémique.

\section{Le climat et son rôle dans l'émergence des maladies}

Les conditions climatiques jouent un rôle dans la dynamique des maladies liées à l'eau (PASCUAL et al., 2002), en particulier par leur forte influence sur les populations de vecteurs dont les gîtes 
larvaires sont mis en eau par les précipitations (MONDET et al., 2005) ou en créant les conditions en température et humidité favorables à une bonne survie des moustiques adultes (GLASSER et GOMES, 2002). Des recherches sur le changement climatique et la santé en Inde ont montré, de manière très générale, les conséquences sur un possible accroissement des risques de transmission du paludisme et, sans doute, d'autres maladies à vecteur (PADMA, 2005; CHAKRAVARTI et KUMARIA, 2005). À Chennai, le nombre de cas de paludisme, de dengue, de choléra et de leptospirose, atteint un pic au cours de la seconde moitié de la saison des pluies. L'émergence des maladies liées à l'eau est très souvent liée aux inondations engendrées par les pluies souvent torrentielles de la mousson, en juin/juillet sur la côte sud-ouest de l'Inde (mousson du Sud-Ouest), en septembre/octobre dans la partie sud-est de l'Inde (mousson du Nord-Est). La leptospirose sévit également dans le monde entier particulièrement dans les pays tropicaux en Asie, en Amérique latine et en Afrique. Elle est également présente, avec une incidence beaucoup plus faible cependant, en Amérique du Nord et en Europe, notamment en France. En Inde du Sud, elle se manifeste régulièrement sous forme de cas d'émergence épidémique liés aux pluies de mousson comme en 2005 et 2006 dans plusieurs États du sud de l'Inde: Orissa, Gujarat, Kerala et Karnataka (JENA et al., 2004 ; PAPPACHAN et al., 2004b).

\section{Conclusions}

Nous sommes donc, en Inde du Sud, dans un milieu où de nombreuses maladies liées à l'eau peuvent exister sous forme endémique, se manifestant plus ou moins cycliquement de manière épidémique, émerger et ré-émerger de façon brutale, comme c'est le cas des arboviroses. L'intensité de telles manifestations épidémiques est fortement liée aux modifications de l'environnement urbain et au constant accroissement des densités de populations (GUPTA et MITRA, 2002). La transformation du rural à l'urbain entraîne des bouleversements des modes de vie des populations humaines et l'apparition de microclimats, liés à de nouveaux biotopes, peut devenir favorable au développement de 
certains vecteurs d'agents pathogènes (BANERJEE, 1996). L'accès aux soins, la représentation systémique du réseau de santé, les données sérologiques, les variations d'abondance spatio-temporelle des populations de moustiques, etc. sont autant de données qui suggèrent une distribution spatiale et temporelle inégale des maladies, arboviroses incluses. Il convient d'étudier l'ensemble des facteurs si l'on veut avoir une influence sur cette dynamique tant en période endémique (inter-épidémique) qu'en période épidémique (SABESAN et Raju, 2005; GUPta et al., 2003) ou pour mesurer les risques d'émergence de maladies rares ou inédites. Ainsi, la connaissance puis l'analyse des changements de cet environnement multiple peuvent-elles permettre d'atteindre, dans une approche intégrée comparative, l'identification des relations existant entre ces phénomènes et les maladies liées à l'eau. Cette intrication et cette interdépendance des pathologies autour d'un facteur dominant, celui de la présence d'eau, établissent dans le milieu urbain en évolution le principe de la pathocénose qui veut que les pathologies concurrentes, dans une population délimitée, sur un territoire et à une période de temps donnés, ont des relations qualitatives et quantitatives qui les définissent. La fréquence et la distribution de chaque pathologie dépendent alors non seulement de facteurs endogènes (pouvoir infectieux, virulence, voie d'infection, type de vecteur) ou exogènes (climat, urbanisation, promiscuité humaine), mais aussi de la fréquence et de la distribution de toutes les autres pathologies présentes dans cette même population (GRMERK, 1969).

Il est clair aujourd'hui qu'il n'est pas de situation épidémiologique en équilibre stable (un des principes de la pathocénose) et l'émergence de maladies inédites le montre. Dans un contexte de développement croissant et face à la globalisation des pathologies, il est urgent de prendre en compte ces dynamiques de l'émergence dans tous les systèmes de santé et sur tous les territoires. Cette première analyse donnerait naturellement les principes déterminants et explicatifs du risque d'émergence, de la transmission et de la dissémination des agents pathogènes, pour identifier les liens existant entre ces différents phénomènes dans des écosystèmes variés et a priori interdépendants. On disposerait ainsi d'arguments pour orienter une recherche/enquête qui pourrait permettre de discerner les relations de causes à effets relatives à la situation 
observée. L'ensemble des données participerait ainsi à l'analyse, en incluant l'allocation des ressources, l'évaluation de l'accès aux soins, la représentation systémique du réseau de santé, les données sérologiques, les variations d'abondance spatio-temporelle des populations de moustiques, les changements de l'environnement, y compris ceux du climat, l'augmentation des possibilités de transport de personnes infectées et (ou) de vecteurs contaminés, l'absence de maîtrise des problèmes liés à l'eau... qui sont, in fine, autant de sujet d'observation. Ces éléments doivent être pris en compte, d'extrême urgence, dans tous les systèmes de santé et par tous les responsables, face à la globalisation des pathologies.

\section{Références bibliographiques}

Afssa, 2006 - Dossier sur les leptospiroses. Agence française de sécurité sanitaire des aliments. Disponible sur: www.afssa.fr

Annual Action Plan, 2003 - Information formats for Urban Malaria Scheme. Annual Action Plan, Corporation of Chennai, Inde.

BANERJEE K., 1996 - Emerging viral infections with special reference to India. Indian J. Med. Res., 103 (4): 177.

BATRA C. P., ADAK T., SHARMA V. P. et al., 2001 - Impact of urbanisation on bionomics of An. culicifacies and An. stephensi in Delhi. Indian J. Malariol., 38: 61-75.

BECK L. R., LOBITZ B. M., WOOD B. L., 2000 - Remote Sensing and Human Health: New Sensors and New Opportunities. Emerging Infectious Diseases, 6 (3) : 217-226.

BoHLE H. G., 2001 - «Vulnerability and Crititicality: Perspectives from Social Geography ». In IHDP Update : Newsletter of the International Human Dimensions Programme on Global Environmental Change : 1-7.

BROOR S., DAR L., SENGUPTA S. et al., 1997 - « Recent dengue epidemic in Delhi, India ". In Saluzzo J.-F., Dodet B. (eds.): Factors in the Emergence of Arbovirus Diseases, Elsevier publications : 123-1217.

BUNCH M., 1997 - The Physical Ecology of Slums in Madras - A GIS Analysis of the 1986 Survey of Slums. The Indian Geographical Journal, 71 (1): 12-32.

CECILIA D., 2004 - « Dengue: the re-emerging disease ». In Mishra A.C. (ed.) : Commemorative Compendium, Pune, India, NIV publications : 278-307. 
Census of India, 2004 - Disponible sur: http://www.censusindia.net/ advance_release_calendar.html

ChakravarTI A., Kumaria R., 2005 - Eco-epidemiological analysis of dengue infection during an outbreak of dengue fever, India. Virol. J., $2(1): 32$.

Chandramouli I. A. S., 2003 - "Slums in Chennai: a profile ". In Bunch M. J., Suresh V. M., Kumaran T. V. (eds.) : Proceedings of the Third International Conference on Environment and Health, Chennai, India, 15-17 December, 2003, Chennai: Department of Geography, University of Madras and Faculty of Environmental Studies, York University: 82-88.

Chung Y. K., PAng F. Y., 2002 - Dengue infection rate in field populations of female Aedes aegypti and Aedes albopictus in Singapore. Trop. Med. Intern. Health, 7 (4): 322-330.

Citizens' water forum, 2006 - http://www.geocities.com/chennaiwaterforum/ CRME, 2003-Annual report, ICMR. Disponible sur: http://www.icmr.nic.in/ annual/crme/2003-04/annual_report.htm

Deepa M., Pradeepa R., Rema M., Mohan A., Deepa R., Shanthirani S., MoHAN V., 2003 - The Chennai Urban Rural Epidemiology Study (CURES) - Study design and methodology (Urban component) (CURES - I). J. Assoc.. Phys. India, 51 : 863-870.

Dhamodaran S., Ananthan S., Kuganantham P., 1995 - A retrospective analysis of the Madras epidemic of non-01 Vibrio cholerae new serogroup 0139 Bengal. Indian J. Med. Res., 101 : 94-97.

Duhamel G., Gombert D., Paupy C., Quatresous 1., 2006 - Mission d'appui à la lutte contre l'épidémie de chikungunya à la Réunion. IGAS (Inspection générale des affaires sociales), Rapport n 2006 012, 59 p.

DUPONT V., 2004 - Socio-spatial differentiation and residential segregation in Delhi: a question of scale? Geoforum, 35: 157-175.

Focks D. A., Brenner R. J., Hayes J., Daniels E., 2000 - Transmission thresholds for dengue in terms of Aedes aegypti pupae per person with discussion of their utility in source reduction efforts. Am. J. Trop. Med. Hyg., 62 (1): 11-18.

Glasser C.M., Gomes A. de C., 2002 - Clima e sobreposição da distribuição de Aedes aegypti e Aedes albopictus na infestação do Estado de São Paulo. Rev. Saúde Pública, 36 (2): 166-172.

GRMERK M. D. 1969 - Préliminaire d'une étude historique des maladies. Annales ESC, 24: 1437-1483.

Gupta I., Mitra A., 2002 - Basic amenities and health in urban India. Natl. Med. J. India, 15 (4) : 242-244. 
GUPTA R., JAY D., JAIN R., 2003 - Geographic Information Systems for the study and control of infectious diseases. Map India Conference (C) GISDevelopment.net, disponible sur: http://www.gisdevelopment.net/ application/health/overview/mi03113.htm

Hawley W. A., 1988 - The biology of Aedes albopictus. J. Amer. Mosq. Control Assoc. (sup.), 4 : 2-39.

Jena A. B., Mohanty K. C., Devadasan N., 2004 - An outbreak of leptospirosis in Orissa, India: the importance of surveillance. Trop. Med. Intern. Health, 9 (9): 1016-1021.

Kröckel U., Rose A., Eiras A. E., Geier M., 2006 - New tools for surveillance of adult yellow fever mosquitoes: comparison of traps catches with human landing rates in an urban environment. J. Am. Mosq. Control Assoc., 22 (2) : 229-238.

Kumar R., Krishnan S. K., Rajashree N. et al., 2003 - Perceptions of mosquito borne diseases. J. Epidemiol. Community Health, 57: 392.

Le choléra, 2006 - Disponible sur: http://www.pasteur.fr/actu/presse/documentation/cholera.htm

Maiti S., AgraWAl P. K., 2005 - Environmental degradation in the context of growing urbanization: a focus on the metropolitan cities of India. J. Hum. Ecol., 17 (4) : 277-287.

Mishra A. C. (ed.), 2004 - Golden Jubilee, Commemorative Compendium. Pune, India, NlV Publications, $427 \mathrm{p}$.

MOHAN V., SHANTHIRANi S., DEEPA R. et al., 2001 - Intra-urban differences in the prevalence of the metabolic syndrome in southern India - The Chennai Urban Population Study (CUPS No. 4). Diabetic Medicine, 18 (4): 280 .

Moloney J. M., Skelly C., Weinstein P. et al., 1998 - Domestic Aedes aegytpi breeding site surveillance: limitations of RS as a predictive surveillance tool. Amer. J. Trop. Med. Hyg., 59 (2) : 261-264.

MONDET B., 1996 - Épidémiologie des arboviroses: utilisation et intérêt de la détermination de l'âge physiologique des femelles de moustiques vecteurs. Bull. Soc. Path. Ex., 89: 155-1160.

Mondet B., Diaite A. Fall A. G., 2005 - Relations entre la pluviométrie et le risque de transmission virale par les moustiques. Cas du virus de la Rift Valley Fever (RVFV) dans le Ferlo, Sénégal. Environnement, Risques et Sante, 4 (2) : 125-9.

MOURYA D. T., YAdav P., Mishra A. C., 2004 - "The current status of Chikungunya virus in India ". In NIV, Commemorative Compendium, Pune, NIV Publications: 265-277. 
Muthusethupathi M. A., Shivakumar S., Suguna R. et al., 1995 Leptospirosis in Madras: a clinical and serological study. J. Assoc. Physicians India, 43 (7) : 456-458.

Myers R. M., Carey D. E., Reuben R., Jesudass E., Deranitz C., MALATI J., 1965 - The 1964 epidemic of dengue-like fever in South India. Isolation of Chikungunya virus from human sera and from mosquitoes. Indian J. Med. Res., 53: 694-701.

PADMA T. V., 2005 - Heat and storms ahead for India, says climate study. Disponible sur: www.scidev.net/News/index.cfm? fuseaction=readnews\&itemid $=2343 \&$ language $=1$

Pappachan M. J., Mathew S., Aravindan K. P. et al. 2004a - Risk factors for mortality in patients with leptospirosis during an epidemic in northern Kerala. Natl. Med. J. India, 17: 240-242.

Pappachan M. J., Sheela M., Aravindan K. P., 2004b - Relation of rainfall pattern and epidemic leptospirosis in the Indian State of Kerala. J. Epidemiol. Community Health, $58: 1054$.

Pascual M. Bouma M. J., Dobson A. P., 2002 - Cholera and climate: revisiting the quantitative evidence. Microbes and Infection, 4:237-245.

Reiter P., Lathrop S., Bunning M. et al., 2003 - Texas lifestyle limits transmission of dengue virus. Emerging Infectious Diseases, 9 (1): 86-89.

RODHAIN F., 1983 - Maladies transmises par les Culicinés et urbanisation: un exemple de co-évolution. Bull. Institut Pasteur, 81 : 33-54.

Rodrigues F. M., Patankar M. R., Barnerjee K. et al., 1972 - Etiology of the 1965 epidemics of febrile illness in Nagpur city, Maharashtra State, India. Bull. Wld. Hlth Org., 46: 173-179.

SABESAN S., RAJU K. H. K., 2005 - GIS for rural health and sustainable development in India, with special reference to vector-borne diseases. Current Sciences, 88 (11): 1749-1752.

SALEM G., 1998 - La santé dans la ville. Géographie d'un espace dense: Pikine (Sénégal). Éditions Karthala et Orstom, Hommes et Sociétés, $360 \mathrm{p}$.

Saluzzo J.-F, Digoutte J.-P., Chartier C., Martinez D., Bada R., 1987 Focus of Rift Valley fever virus transmission in southern Mauritania. Lancet, 1 (8531) : 504.

Sauerborn R., Adams A., Hien M., 1996 - Household strategies to cope with the economic costs of illness. Soc. Sci. Med., 43 (3) : 291-301.

Strickman D., Kittayapong P., 2002 - Dengue and its vectors in Thailand: introduction to the study and seasonal distribution of Aedes larvae. Am. J. Trop. Med. Hyg., 67 (3) : 247-259. 
Teixeira M. G., Barreto M. L., Costa M. C. N. et al., 2002a - Sentinel areas: a monitoring strategy in public health. Cad. Saúde Pública, 18: 1193-1195.

Teixeira M. G., Barreto M. L., Costa M. N. et al., 2002b - Dynamics of dengue virus circulation: a silent epidemic in a complex urban area. Tropical Medicine E International Health, 7 (9): 757-762.

Tewari S. C., Thenmozhi V., Katholi C. R., Manavalan R., Munirathinam A., Gajanana A., 2004 - Dengue vector prevalence and virus infection in a rural area in South India. Trop. Med. Intern. Health, 9 (4): 499-507.

Trape J.-F., Lefebvre-Zante E., legros F., Ndiaye G., Bouganali H., DRUILHE P., SALEM G., 1992 - Vector density gradients and the epidemiology of urban malaria in Dakar, Senegal. Am. J. Trop. Med. Hyg., 47 (2) : $181-189$.

UN-Habitat, 2006 - Disponible sur: http://www.unhabitat.org/about/challenge.asp

VCRC, 2004 - VCRC Annual report. http://icmr.nic.in/annual/annual.htm

Vijayakumar T. S., Chandry S., Narayanan S. et al. 2005 - Is dengue emerging as a major public health problem ? Indian Journal of Medical Research, 121: 100-107.

WHO, 2003 - Human leptospirosis: guidance for diagnosis, surveillance and control. Doc. WHO, ISBN 924154589 5, 121 p.

WORK T. H., TRAPIDO H., 1957 - Kyasanur Forest Disease, a new virus disease in India: summary of preliminary report of investigations of the VRC on the epidemic disease affecting forest villagers and wild monkeys of Shimoga district, Mysore State. India J. Med. Sci., 11:341342. 



\section{Risques sanitaires et gestion des eaux usées et des déchets à Kribi (Cameroun)}

René Joly ASSAKO ASSAKO

Carine Alix DJILO TONMEU

Daniel BLEY

Si les pays actuellement développés ont pu mettre en place des systèmes d'assainissement qui restent certes à parfaire, il est à noter que dans les villes d'Afrique, la question d'une gestion efficace des déchets en général et des eaux usées en particulier représente encore une des préoccupations majeures des autorités municipales et des populations qui y résident (DORIER-APPRILl, 2006). En effet, les déchets sont généralement déversés dans la nature sans traitement préalable, parfois même dans le voisinage immédiat des lieux d'habitation. Compte tenu de la complexité de cette question et pour éviter une approche trop générale qui ne tiendrait pas compte des particularités locales, nous nous intéresserons aux eaux usées et aux ordures ménagères qui contiennent des produits toxiques, une forte charge en agents pathogènes et en parasites de toutes sortes et qui en polluant les milieux de vie, de loisirs et de consommation exposent les populations à un risque sanitaire permanent.

Pour saisir les différentes facettes de ce risque sanitaire et réfléchir à une meilleure gestion des déchets, il nous a paru nécessaire 
d'adopter une démarche pluridisciplinaire qui associe des géographes qui s'intéressent à l'impact du milieu et à la spatialisation des zones à risques, mais aussi des anthropologues qui peuvent dans le même temps analyser les pratiques et les représentations des populations en matière d'hygiène. Parmi l'ensemble des interrogations qui peuvent émerger d'une telle recherche, trois questions nous semblent essentielles: la gestion hasardeuse des déchets à Kribi relève-t-elle de la pauvreté urbaine, de l'incivisme des populations ou de l'inefficacité du système d'assainissement ? La configuration géomorphologique des sites urbains est-elle prise en compte dans le dispositif infrastructurel de l'assainissement? $\mathrm{Au}$ regard du risque sanitaire vécu à l'interface des pratiques sociales (mode de vie), et des pratiques spatiales (utilisation de l'espace aussi bien pour le dépôt que pour le transport des ordures), quels pourraient être les apports respectifs mais complémentaires de la géographie et de l'anthropologie dans une recherche dont l'objectif est de proposer un système sécurisé de la gestion des déchets?

Après avoir présenté la zone d'étude, la notion de risque sanitaire est analysée suivant une approche multivariée. Nous faisons ensuite le point sur l'état actuel de la gestion des eaux usées et des ordures ménagères à Kribi pour proposer, in fine les articulations d'un modèle prenant davantage en compte la santé des populations.

\section{Présentation de la zone d'étude}

Kribi est une ville balnéaire moyenne du Sud-Cameroun, située entre $2^{\circ} 56^{\prime}$ et $3^{\circ} 02^{\prime}$ de latitude nord et $9^{\circ} 54^{\prime}$ et $9^{\circ} 57^{\prime}$ de longitude est (fig. 1). Elle se retrouve dans les basses terres côtières du littoral camerounais avec une altitude moyenne de $20 \mathrm{~m}$ (Schéma de développement local de la ville de Kribi, 1998). Le site bâti couvre une superficie d'environ 14470 hectares, avec une population cosmopolite estimée à 40000 habitants en 2004. Sur le plan climatique, elle bénéficie de la variante maritime du climat 
Figure 1.

Kribi, une ville balnéaire ouverte sur l'océan Atlantique.

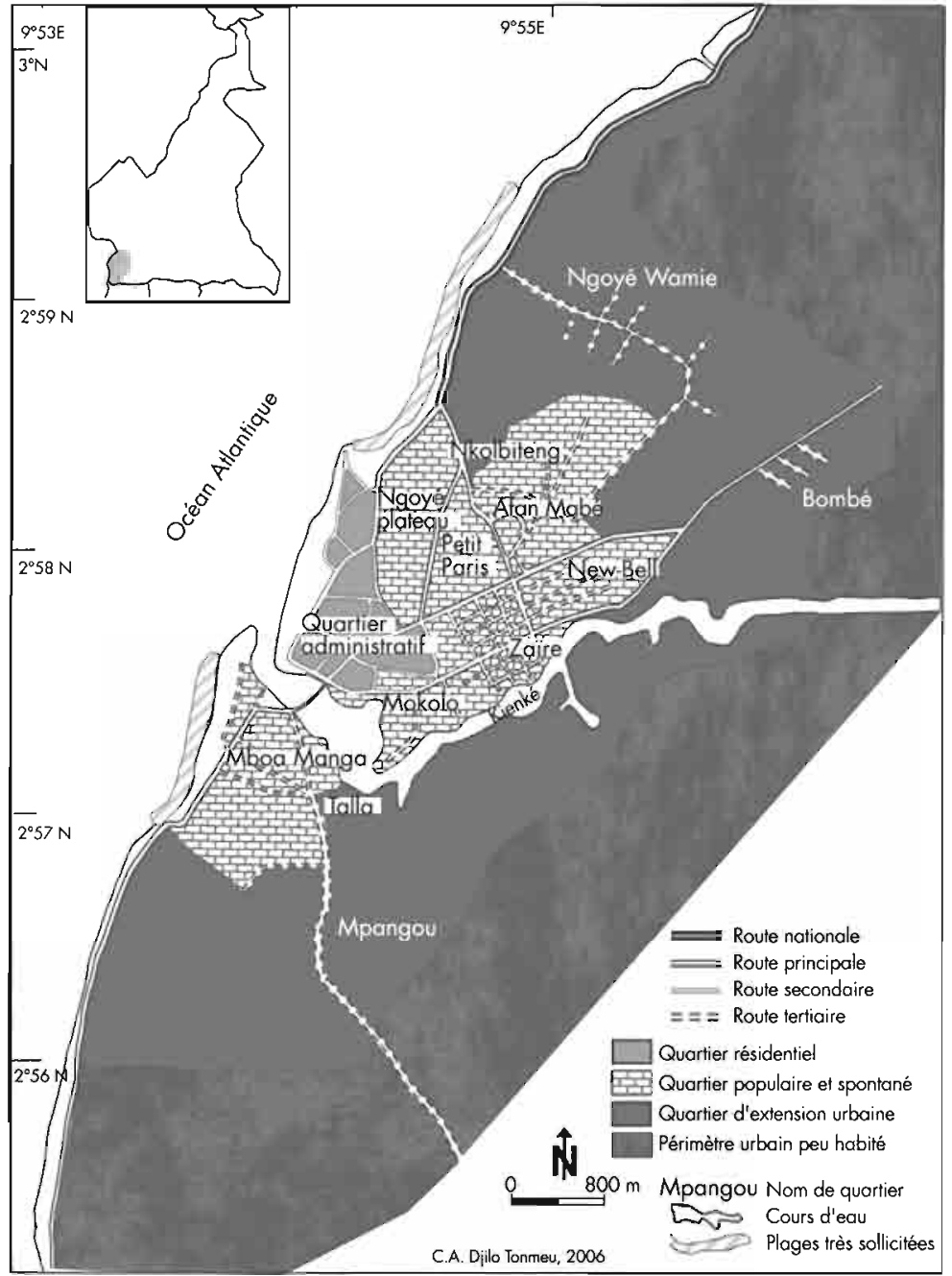

équatorial humide, avec quatre saisons. Les précipitations y sont constantes et on enregistre en moyenne $2900 \mathrm{~mm}$ par an. Les températures oscillent entre $24,15^{\circ} \mathrm{C}$ et $27,88^{\circ} \mathrm{C}$. L'activité économique de Kribi est dominée par les trois secteurs que sont le commerce, la pêche et le tourisme. 


\section{La gestion hasardeuse des déchets dans la ville de Kribi}

L'analyse qui suit concerne deux types de déchets: les ordures ménagères et les eaux usées domestiques auxquelles on pourrait ajouter les déchets d'origine industrielle pouvant avoir un impact sur la santé des populations.

\section{La gestion des ordures ménagères}

Contrairement à l'impression de salubrité que donne le quartier administratif et résidentiel, la quasi-totalité de Kribi connaît de grosses difficultés d'évacuation des ordures ménagères. Deux filières coexistent: le système municipal de collecte et la gestion individuelle.

La commune urbaine de Kribi dispose d'un service de ramassage des ordures grâce à deux camions qui passent deux à trois fois par semaine dans les quartiers pourvus de voies carrossables le long desquelles les populations déposent une grande variété de récipients contenant des ordures: seaux, paniers en fibres végétales, demi-fûts, etc. On compte aussi quelques bacs à ordures installés aux grands carrefours, notamment dans le quartier administratif et près des marchés. Lorsque ces différents récipients sont pleins ou lorsqu'il n'en existe pas, les populations déversent leurs ordures à même les trottoirs. Chargées dans les camions municipaux, ces ordures sont ensuite déversées à la décharge municipale située au quartier Mpangou, dans la banlieue sud-est de Kribi, à $5 \mathrm{~km}$ du centre-ville où elles ne subissent aucun traitement. Dans le meilleur des cas et lorsque le temps le permet, elles sont brûlées: il s'agit donc d'une incinération sauvage, sans aucun équipement ni aménagement adéquats (planche 1).

Une récente enquête menée à Kribi a en effet montré que $10 \%$ des déchets produits dans les quartiers résidentiels, $42 \%$ dans les quartiers populaires et $87 \%$ dans les quartiers périphériques de cette ville échappent au ramassage par les camions de la municipalité (Meva'A Авомо, 2006). Il en ressort que la salubrité varie en 
Planche 1.

Le réseau moderne de collecte des ordures ménagères.

Dans les quartiers de Kribi pourvus d'une bonne desserte routière, les populations déposent leurs ordures dans des demi-fûts (1a), des récipients de récupération en plastique ou en fibres végétales ( $1 b, 1 c$ et $1 d$ ) le long des routes d'où le camion de la mairie les achemine à la décharge de Mpangou (1e).

Mais cette décharge n'est en réalité qu'une clairière dégagée dans la banlieue forestière sud-est de Kribi, sur un terrain plat. Et, du fait de la régularité des pluies, elle s'est transformée en un étang artificiel d'eau sale propice au développement d'une multitude de vecteurs et d'agents pathogènes de maladies tropicales ( $1 \mathrm{f}$ ).
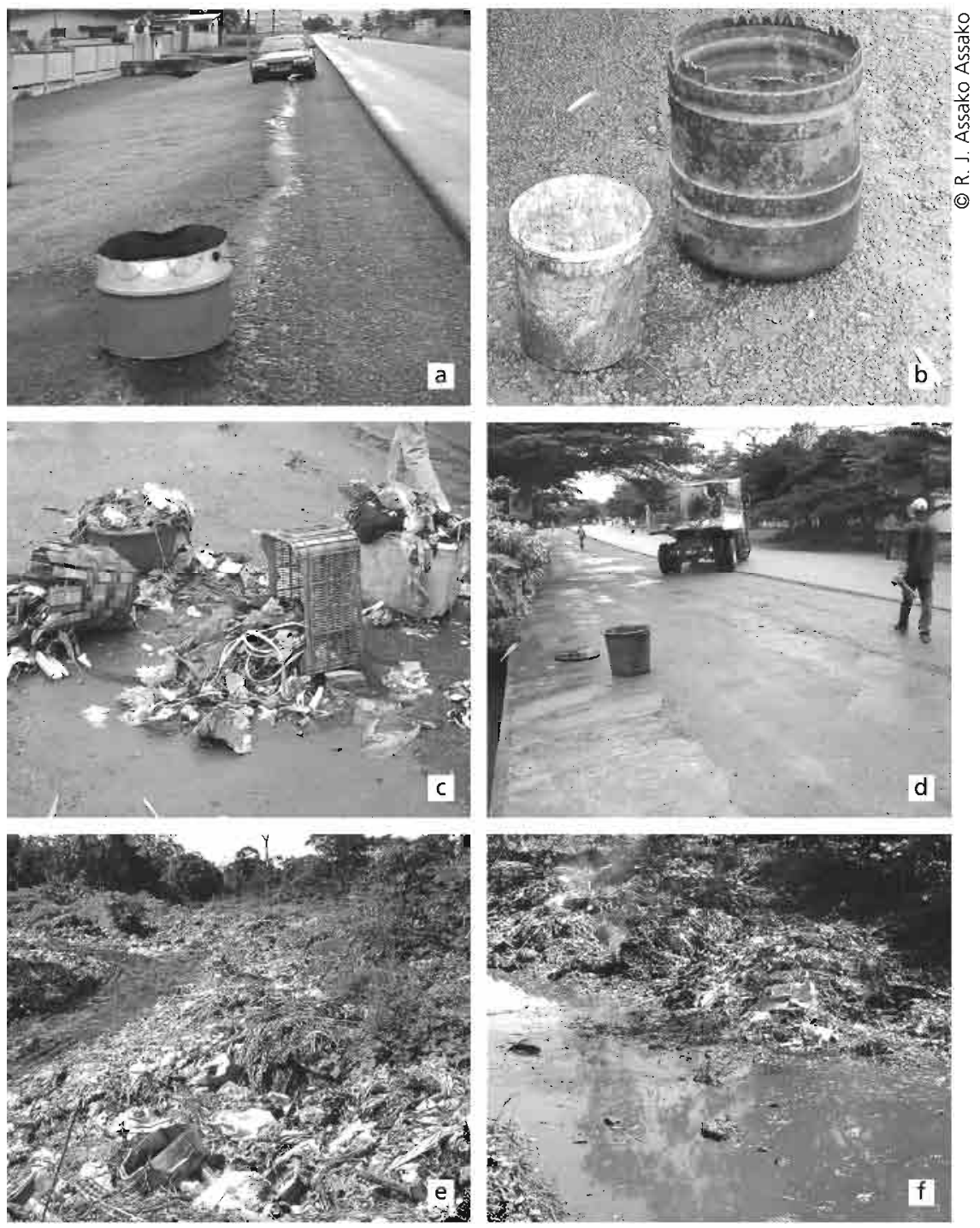
Planche 2.

Gestion individuelle anarchique des ordures ménagères et pollution des cours d'eau à Kribi.

Dans les quartiers populaires enclavés, les populations jettent leurs ordures ménagères derrière leurs habitations où celles-ci peuvent servir d'engrais naturels pour la culture du bananier ou des plantes à condiment (2a). Les berges et les lits des cours d'eau sont également utilisés (2b). C'est là où la mauvaise gestion des ordures ménagères rejoint celle des eaux usées domestiques dans le processus de pollution organique. Les ménages des quartiers populaires ne disposent bien souvent que de latrines à ciel ouvert (2c). La matière fécale stockée dans ces latrines est généralement évacuée vers les cours d'eau voisins au moyen des canalisations aménagées à cet effet ou à l'occasion des pluies. II résulte de cet état de fait que les cours d'eau transurbains présentent souvent à leur embouchure un lit vaseux et de couleur ocre, à cause d'une forte charge de matières organiques en suspension (2d).
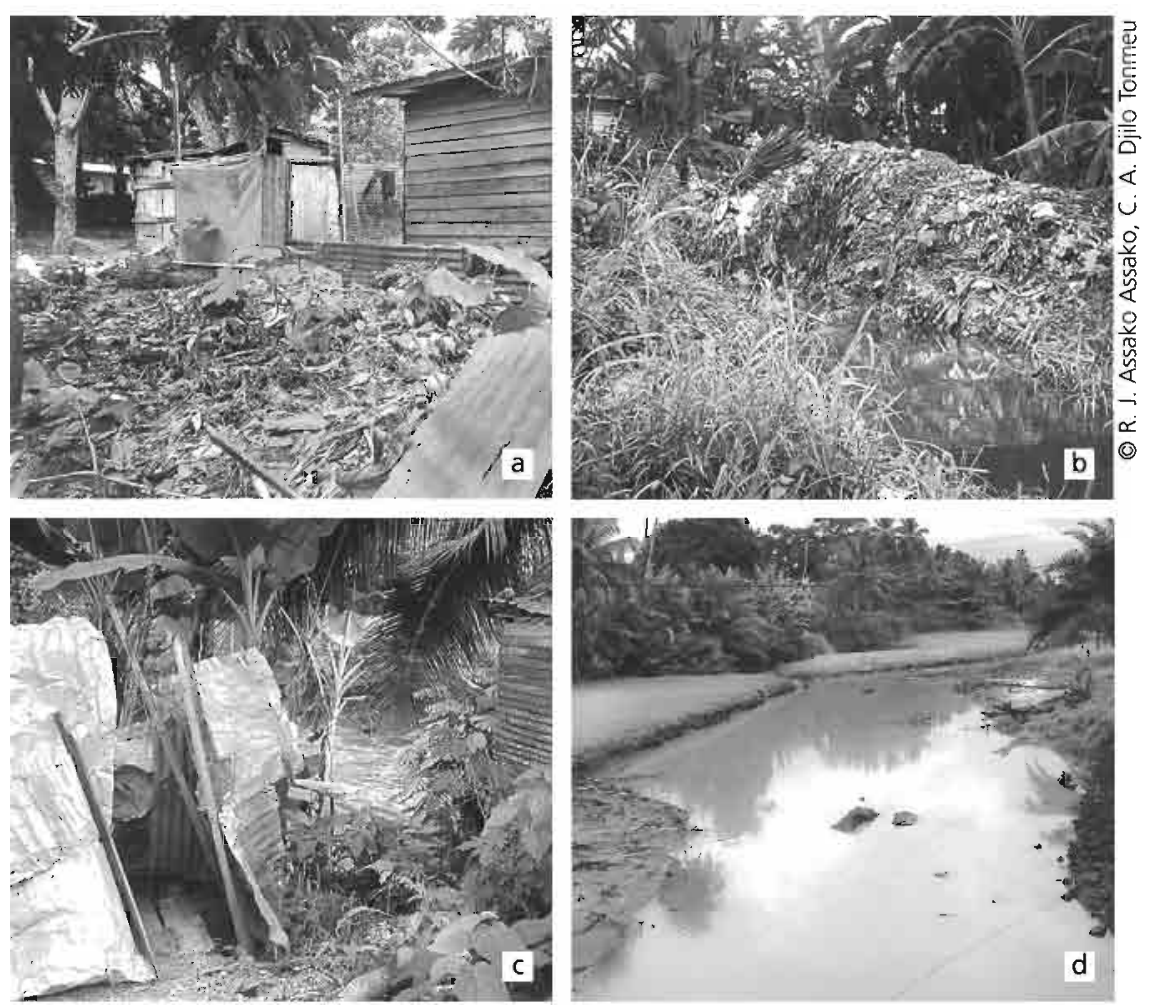

fonction du type de quartier et de la qualité de la desserte routière. La présence de nombreux tas d'ordures, de demi-fûts de collecte abandonnés illustre bien la difficulté de gestion des ordures ménagères à Kribi. 
L'irrégularité du passage des camions de ramassage des ordures, l'insuffisance du matériel de ramassage et des personnels, ainsi que le manque de voies de desserte dans les quartiers populaires comme Afan Mabé, Talla ou Dombé aggravent la situation. Pourtant, c'est dans ces quartiers qu'on enregistre les densités de populations les plus fortes, ce qui se traduit par la production d'énormes quantités d'ordures ménagères qui pourrissent sur place, surtout en saison des pluies.

En marge de ce système municipal, on constate que les populations déversent leurs déchets non loin des lieux d'habitation, dans les rigoles, sur le trottoir, dans les touffes d'herbes. On trouve parfois des tas d'immondices à côté d'un bac à ordures vide et les populations disent que cela facilite la tâche des mendiants et des récupérateurs qui se fournissent dans ces tas d'ordures. Cette situation est observable en tout temps dans les quartiers populaires d'habitat précaire tels que Afan Mabé, Mokolo, Dombé, Petit-Paris où il existe une grande promiscuité. La fréquence des pluies et la faiblesse des altitudes font que ces ordures finissent par rejoindre les cours d'eau. Ces déchets, ainsi que le montre la planche 2, ont un double impact. D'une part, ils constituent du fumier pour les jardins potagers qu'on retrouve aux alentours des maisons et d'autre part, ils contribuent pour beaucoup à la pollution des cours d'eau, du fait de la décomposition des matières organiques.

\section{La gestion des eaux usées}

L'eau représente un élément important dans la vie socioculturelle et économique des peuples de la côte. Les cours d'eau sont des endroits très utilisés par les populations (Batanga, Ngoumba, Mabéa, etc.) pour les baignades, la vaisselle, la lessive, la pêche, et même pour des cérémonies traditionnelles. Cependant, sa mauvaise utilisation et sa mauvaise gestion peuvent la rendre dangereuse pour les populations.

Il existe plusieurs modes d'approvisionnement en eau à Kribi. La diversité de ces sources d'approvisionnement fournit une eau souvent impropre à la consommation et présentant des risques sanitaires plus ou moins importants. La ville est approvisionnée 
en eau potable à partir d'une station de pompage et de traitement située près de la rivière Kienké qui en est la source, au quartier Dombé. Kribi connaît de véritables problèmes d'approvisionnement en eau potable, liés à la défaillance du réseau de distribution d'eau de l'ex-Société nationale des eaux du Cameroun (Snec), devenue Camwater à l'issue de sa privatisation. Les populations s'approvisionnent généralement plutôt dans les puits, les forages, les rivières, et dans une moindre mesure aux bornes-fontaines. L'eau ainsi recueillie sert à tous les usages, y compris la boisson et la cuisson des aliments (fig. 2). Il est important de noter que seulement un quart des foyers dispose de l'eau courante, que $14,8 \%$ des individus continuent de consommer l'eau du puits et $6,1 \%$ l'eau des cours d'eau, et dans une moindre mesure l'eau de forage sans souci de la qualité.

Ces puits, situés dans la cour, derrière la maison et même à proximité des latrines, sont généralement mal ou pas du tout protégés.

L'approvisionnement en eau potable constitue un problème majeur de santé à Kribi. C'est la non-protection de ces sources d'approvisionnement en eau qui fait problème. Les inondations récurrentes

Figure 2.

Estimation des volumes d'eau et des usages par ménage et par jour (d'après les données d'enquêtes de D. Meva'A Авомо, 2006).

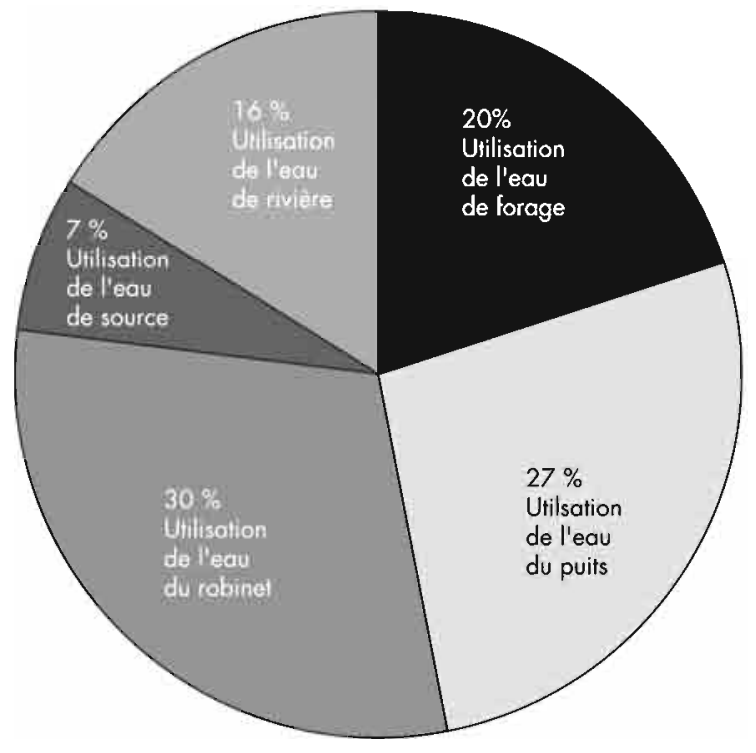


Figure 3.

Altitude et hydrographie: deux composantes géographiques déterminantes dans l'organisation de l'assainissement à Kribi.

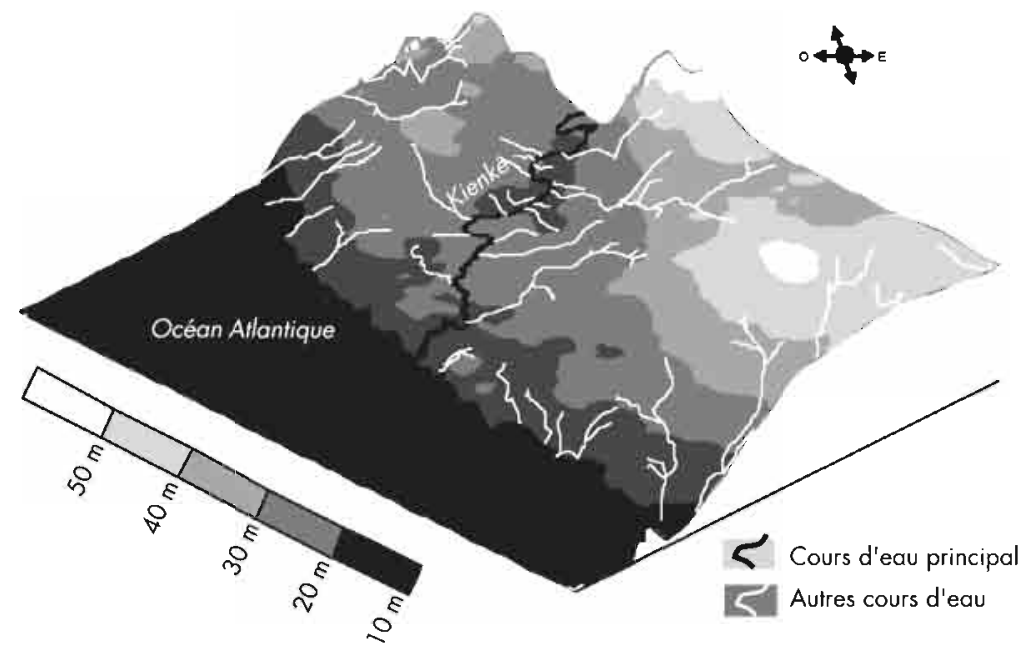

Figure 4.

Configuration topographique générale du site de Kribi:

une donnée fondamentale pour le choix d'implantation des décharges et des industries polluantes.

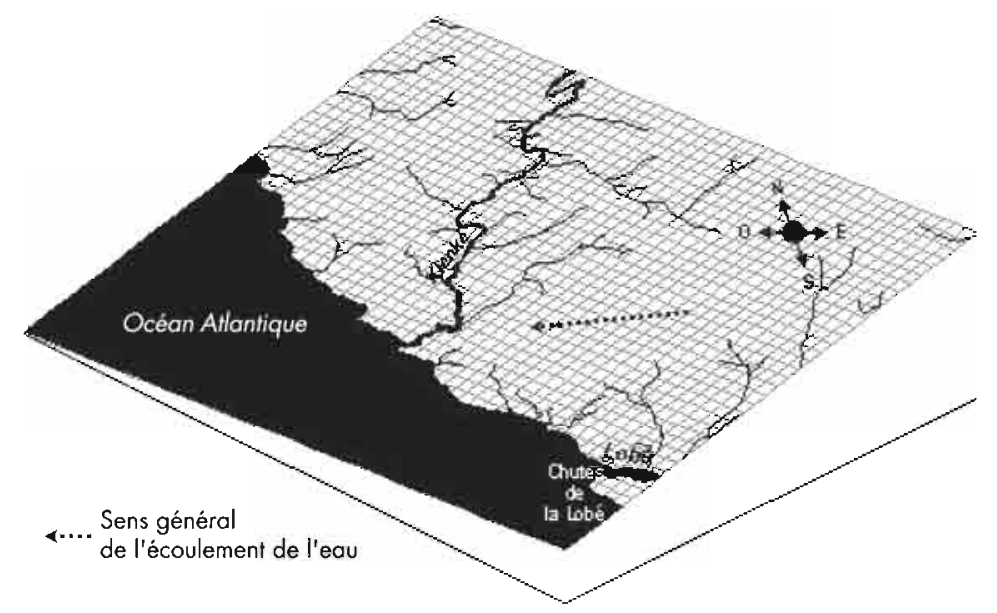

liées non seulement à la configuration géomorphologique du site mais aussi à la densité du réseau hydrographique (fig. 3 et 4 ) et à la forte pluviométrie font qu'il y a une communication osmotique quasi permanente entre les points de ravitaillement en eau (cours d'eau et puits) et les effluents urbains (latrines, caniveaux, décharges, etc.) 
La ville de Kribi ne dispose pas de réseau d'assainissement adéquat. L'évacuation des eaux usées se fait généralement de façon individuelle. Les menaces et pénalités n'étant imposées que sporadiquement aux particuliers et presque jamais aux unités de production ou de transformation des matières premières par le service d'hygiène, la gestion des eaux usées ne semble pas préoccuper les autorités municipales. Celles produites par les ménages sont déversées dans la nature dans tous les quartiers d'habitat précaire comme Afan Mabé, Petit-Paris, Mokolo, etc. En dehors de quelques habitations qui disposent de fosses d'aisance modernes (environ 15\%), la plupart des populations versent leurs eaux usées dans la cour ou encore dans les latrines traditionnelles mal aménagées et à ciel ouvert. La platitude du terrain et la présence de multiples dépressions géomorphologiques font que les eaux usées domestiques déversées dans la nature, évidemment sans aucun traitement, créent de nombreux points de stagnation où se développent une kyrielle de germes pathogènes, de parasites et des vecteurs de maladies infectieuses (cf. Annexe). Ces quartiers populaires présentent par conséquent les risques sanitaires les plus élevés, en rapport avec les eaux usées et les ordures ménagères.

S'agissant des maisons modernes, généralement implantées dans le quartier administratif, leurs fosses septiques sont vidangées par les sociétés privées de vidange en provenance de Douala. ces déchets sont acheminés vers la décharge des eaux usées du Bois des Singes à Douala (ASSAKO ASSAKO, 2005), même s'il n'est pas impossible que certains de ces camions soient vidangés dans les fleuves et rivières qui traversent la route Kribi-Douala comme la Sanaga, la Dibamba ou le Nyong. La ville de Kribi ne dispose pas encore d'une station d'épuration des eaux usées et comme nous l'avons constaté avec la gestion des ordures ménagères, même si les modes d'évacuation des eaux varient selon le type de quartier, il existe une gestion anarchique généralisée dans la ville (tabl. 1).

Au regard de ce qui précède, la gestion anarchique de l'eau compte bien parmi les facteurs de risque sanitaire. En effet, les canalisations diffuses dont les exutoires sont à l'air libre, couplées à la faible altimétrie et à la faible perméabilité des sols, favorisent la stagnation des eaux de surface. Telle est aussi la réalité des cours d'eau dont l'obstruction des lits ralentit l'écoulement de l'eau, déjà considérablement freinée par la planimétrie des bas-fonds. 
Tableau 1.

Modes d'évacuation des eaux usées par type de quartier

(d'après D. MEVA'A АвоMO, 2006)

\begin{tabular}{|lllcr|}
\hline $\begin{array}{l}\text { Type } \\
\text { de } \\
\text { quartier }\end{array}$ & Désignation & $\begin{array}{c}\text { Lieux } \\
\text { d'évacuations }\end{array}$ & $\begin{array}{c}\text { Nombre } \\
\text { de ménages } \\
\text { enquêtés }\end{array}$ & Pourcentage \\
\hline Résidentiel & Quartier & Dans la cour & 5 & 25 \\
& administratif & Dans les rigoles & 6 & 30 \\
& & Derrière la maison & 8 & 40 \\
& & Cours d'eau & 1 & 5 \\
& Total & $\mathbf{2 0}$ & $\mathbf{1 0 0}$ \\
\hline Populaire & - Afan Mabé & Dans la cour & 14 & 28 \\
& - Mokolo & Dans les rigoles & 3 & 6 \\
& - Petit-Paris & Derrière la maison & 24 & 48 \\
& & Cours d'eau & 9 & 18 \\
& & Total & $\mathbf{5 0}$ & $\mathbf{1 0 0}$ \\
\hline Périphérique & - Talla & Dans la cour & 4 & 13,3 \\
& - Mpangou & Dans les rigoles & 2 & 6,7 \\
& & Derrière la maison & 23 & 76,7 \\
& & Cours d'eau & 1 & 3,3 \\
& & Total & $\mathbf{3 0}$ & $\mathbf{1 0 0}$ \\
\hline
\end{tabular}

\section{Déchets industriels et risques sanitaires à Kribi}

Kribi dispose de nombreux hôtels et des installations relatives au terminal du pipeline Tchad-Cameroun. Le fonctionnement de tous ces équipements n'est pas sans danger sur l'environnement et sur la santé des populations. En observant le mode de gestion des déchets dans les hôtels, on peut constater qu'il y a un effort de fait en matière de respect de l'environnement. Les déchets solides sont emballés dans de grands sacs avant d'être transportés par les camions de la municipalité. Les déchets liquides sont déversés dans les fosses septiques et sont collectés par les camions de vidange. Mais cela ne vaut que pour les plus grands de ces hôtels. En effet, les petits hôtels, très nombreux à Kribi, utilisent des fosses à ordures qui présentent un risque certain de pollution non seulement des sols mais aussi de la nappe d'eau souterraine qui se trouve à quelques mètres de profondeur, voire à quelques dizaines de centimètres par endroits. S'agissant des eaux usées, leur mode d'évacuation est identique à celui en usage 
dans les ménages des quartiers populaires avec l'ouverture des regards des fosses à l'occasion des grosses pluies. Cette pratique présente des risques de pollution du sol et des eaux des rivières et de l'océan, les premières étant les canaux naturels de ruissellement et le second étant l'exutoire, la base du bassin hydrographique de Kribi.

Figure 5.

Localisation de la station de réduction de pression du pipeline et de la décharge municipale.

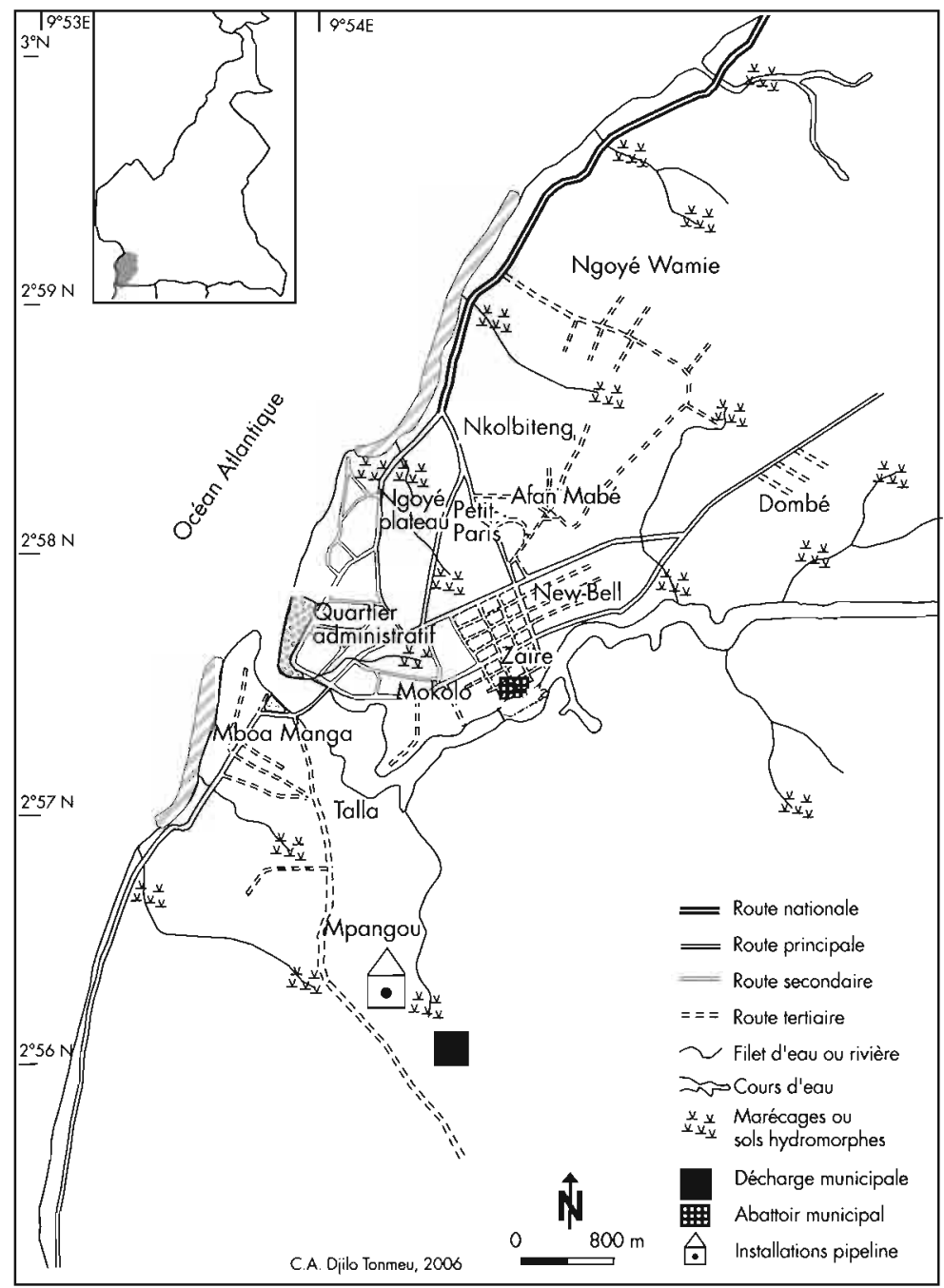


D'autre part, l'une des stations de réduction de pompage et de traitement du pétrole du pipeline Tchad-Cameroun se trouve au sud-est, à la sortie de la ville. Cette station est située à $300 \mathrm{~m}$ de la décharge municipale, dans un quartier où courent de nombreuses rivières comme dans toute la ville (fig. 5). Malgré toutes les dispositions prises par l'entreprise pour limiter les risques de pollution, on enregistre de temps en temps des fuites de pétrole brut qui se déversent dans les rivières environnantes qui charrient ces substances jusqu'à la mer. Car, l'écoulement des rivières et des cours d'eau dans la ville se fait dans le sens est-ouest, c'est-à-dire, de l'intérieur vers l'océan (fig. 3 et 4). Certaines rivières se jettent directement dans la mer, tandis que d'autres rejoignent la Kienké, avant de se jeter dans la mer. Ces substances ont des effets néfastes sur la qualité de l'eau de mème que sur les mollusques et crustacés qu'on y trouve.

Ce qui vient d'être dit au sujet de la station de pompage est également valable pour le robinet de chargement des bateaux situé au large de Kribi, à quelques kilomètres de la plage. Il est possible, à terme, que des fuites de pétrole brut s'y produisent. Sans qu'on puisse déjà établir un lien de causalité, les pêcheurs parlent d'une diminution sensible des prises depuis la mise en service du terminal pétrolier.

\section{Les risques sanitaires liés à la mauvaise gestion des ordures ménagères et des eaux usées}

De façon générale, le risque peut être considéré comme tout danger important menaçant un groupe humain, soit du fait d'un phénomène naturel (on parle de risque naturel), soit du fait de l'action même de l'homme (on parle alors de risque artificiel ou technologique). Ce distinguo a bien été mis en évidence dans le Dictionnaire géographique de P. BAUD et al. (2003). En effet, si les premiers relèvent de la géodynamique propre (éruptions volcaniques, érosion, inondations, etc.), les seconds sont quant à eux 
liés aux évolutions technologiques, ou encore aux comportements de l'homme vis-à-vis de son environnement. Il en est ainsi des mécanismes de pollution sur lesquels nous reviendrons plus loin.

Autant, pour des besoins analytiques, on peut séparer les catastrophes naturelles des catastrophes anthropiques et (ou) technologiques, autant, à l'épreuve des faits, il devient illusoire de les traiter séparément, compte tenu de l'imbrication des phénomènes géographiques et de cette particularité que ceux-ci ont de s'influencer mutuellement. Ce même constat a été fait par A. DAGORNE et R. DARS (1999), qui remarquaient fort opportunément que les risques naturels et artificiels sont intimement liés, tantôt par leurs causes, tantôt par les facteurs d'aggravation des dégâts, tantôt enfin par les conséquences des uns et des autres. Des actions humaines peuvent provoquer une catastrophe naturelle et inversement: l'imperméabilisation d'un front de mer densément peuplé peut rendre une banale inondation catastrophique par l'ampleur des dégâts qui pourront en résulter. De même, la rupture d'un barrage peut entraîner une inondation en aval.

Dans cette étude, nous considérons le risque sanitaire comme une menace permanente d'occurrence des affections pathogènes ou parasitaires engendrées par des pratiques humaines inadéquates en matière de gestion du cadre de vie, plus particulièrement des ordures ménagères et des eaux usées. Le risque sanitaire peut ainsi être considéré comme l'exposition des populations urbaines aux différentes maladies dont la transmission est le fait d'agents pathogènes ou de vecteurs ayant un lien avec les ordures ménagères ou les eaux usées.

À l'instar de la plupart des villes moyennes d'Afrique, Kribi présente de réels et préoccupants risques sanitaires du fait du développement des quartiers spontanés d'habitat précaire, d'une pauvreté urbaine quasi généralisée et de l'insuffisance (voire de l'absence) des systèmes d'assainissement et de gestion des déchets. Les eaux usées domestiques et les ordures ménagères sont, comme le montre le tableau en annexe, porteuses d'une multitude d'agents pathogènes.

\section{Le risque de maladies hydriques}

La ville de Kribi est sous la menace permanente des maladies d'origine hydrique. Il s'agit des maladies dont les agents pathogènes 
se développent dans de l'eau souillée par des déchets humains, animaux ou chimiques. Les milieux insalubres et les eaux souillées sont des réceptacles de germes de nombreuses maladies telles que le choléra, les gastro-entérites, la dysenterie amibienne, les helminthiases, etc. (cf. Annexe). Les enquêtes auprès des ménages et les statistiques médicales nous ont révélé qu'après le paludisme, les maladies d'origine hydrique représentent la première raison de consultation médicale (cas de diarrhées et gastro-entérites, fig. 6). Les épidémies de choléra ne sont pas rares à Kribi. Celle de 2004, qui fut en réalité une excroissance de l'épidémie déclenchée à Douala et qui a fait de nombreuses victimes, reste encore dans les mémoires. Elle a particulièrement touché les quartiers Mokolo, Dombé, et Afan Mabé.

\section{Figure 6.}

Répartition des maladies récurrentes à Kribi (d'après les données du service d'accueil de l'hôpital de district de Kribi, année 2004).

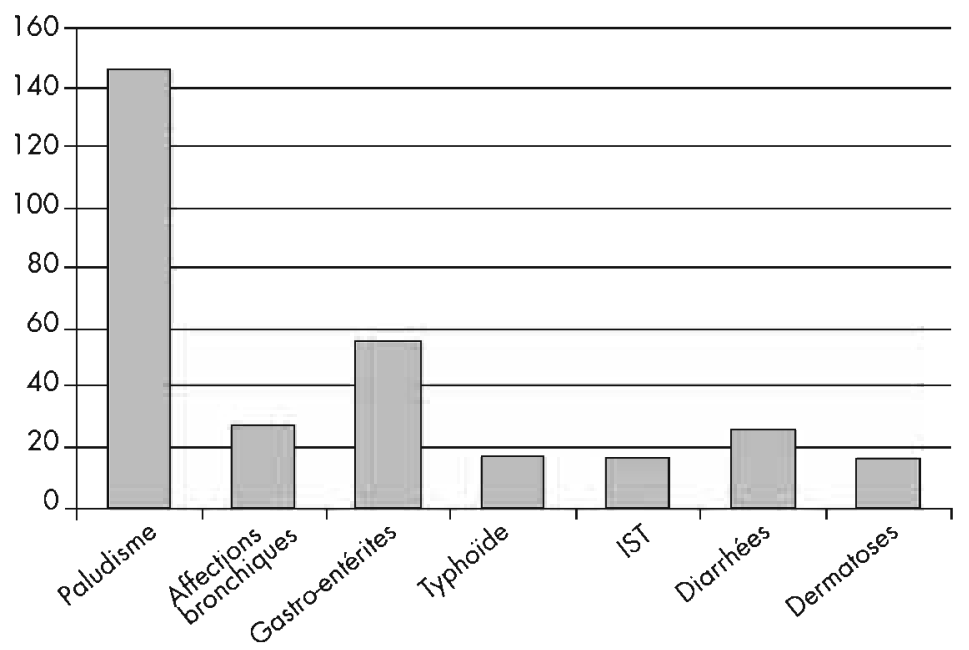

On y enregistre aussi de nombreux cas de dermatoses, infections de la peau, liées au contact avec de l'eau souillée, aussi bien par les eaux usées domestiques que par les hydrocarbures, lors des baignades, de la pêche, de l'extraction du sable par immersion ou des lessives. Il convient de noter que la distribution spatiale de ces maladies permet de constater que c'est dans les quartiers densément peuplés, situés soit dans les marécages, soit au bord d'une rivière tels que Mokolo, New-Bell, Zaïre, Dombé, Afan Mabé 
Figure 7.

Distribution des malades en fonction de leurs lieux d'habitation.

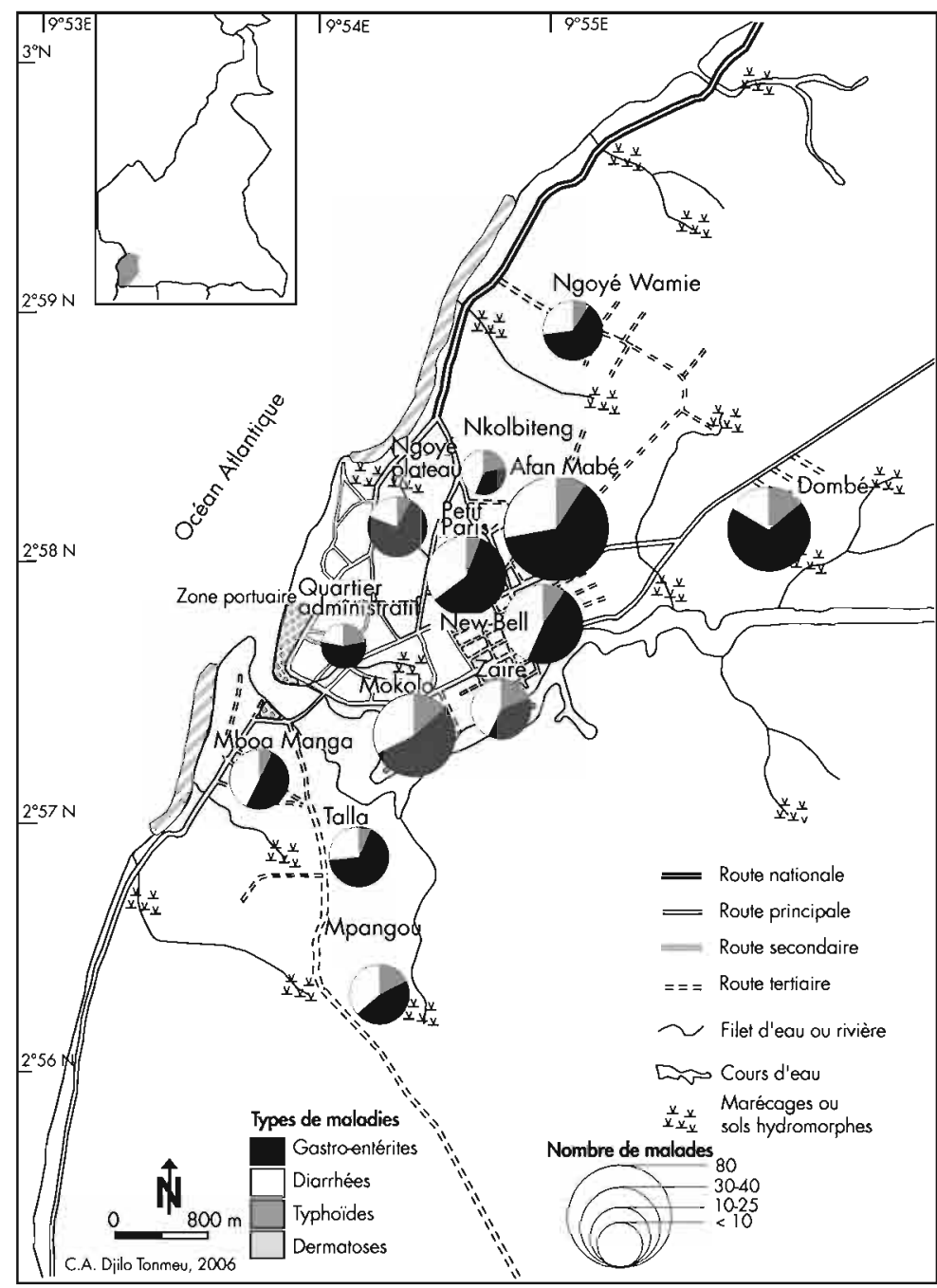

qu'on enregistre le plus grand nombre de malades (fig. 7). Ces quartiers font l'objet d'une grande promiscuité augmentant le risque de transmission des coliformes fécaux.

Nos enquêtes nous ont permis de constater que les déparasitants ou les antihelminthiques sont les principaux médicaments achetés dans la rue. Cette situation nous montre que les populations sont continuellement menacées à Kribi par les maladies du péril fécal. 
Ces conséquences peuvent aussi affecter la pêche locale. Les rivières sont sollicitées par les populations pour la pêche et le ramassage des crustacés. Étant donné que ces rivières doivent posséder de grandes charges de coliformes fécaux, la consommation de ces crustacés augmente le risque de contamination, surtout de ceux qui se mangent crus.

De par son relief plat (altitude moyenne de $20 \mathrm{~m}$ dans le périmètre bâti), son sol argilo-sableux et sa localisation dans les basses plaines côtières avec un faible taux d'infiltration des eaux de pluies, la ville de Kribi est prédisposée à une contamination rapide des eaux de surface et même souterraines. En effet, l'approvisionnement et la consommation de l'eau se font généralement dans les puits qu'on retrouve dans près de $75 \%$ des ménages à Kribi. Ces puits, ainsi que nous l'avons signalé plus haut, sont peu profonds (moins de 5 mètres de profondeur) et mal ou pas du tout aménagés. La configuration du site favorise une contamination par les eaux de ruissellement et la consommation de l'eau qui en ressort peut être un danger pour la population. Cette situation a été à l'origine de l'épidémie de choléra qui a eu de grandes répercussions négatives sur l'ensemble du pays et qui est partie de la consommation par une famille du quartier populaire de Bépanda à Douala de l'eau d'un puits situé à proximité immédiate d'une porcherie traditionnelle (ASSAKO ASSAKO et al., 2005; ASSAKO ASSAKO, 2006).

Cette menace a amené la municipalité de Kribi à organiser les opérations de désinfection des puits de la ville avec de l'hypochlorite de calcium. Cependant, cette initiative connaît encore la réticence des populations qui refusent de payer la somme demandée pour l'assainissement des puits. Dans une pareille situation, des épidémies peuvent surgir continuellement avec des issues fatales.

L'insuffisance de l'assainissement ajouté à celui du ramassage des déchets génère de nombreux problèmes de santé. On enregistre dans cette ville de nombreux cas de schistosomiases, de choléra, et autres helminthes intestinaux liés à la mauvaise utilisation de l'eau. Ces maladies constituent tout autant que le paludisme des charges de morbidité importante pour les populations.

Face aux risques sanitaires permanents, on peut s'interroger pour savoir si le système de santé mis en place peut apporter des solutions concrètes en cas d'épidémie. 


\section{Le système de santé à Kribi}

À Kribi, le nombre de formations sanitaires de la ville peut laisser à penser qu'il est possible de satisfaire la demande en soins (cf. fig. 8), mais il faut cependant noter que ces infrastructures souffrent pour la plupart d'un sous-équipement en matériel et en personnel. L'hôpital de district est le seul hôpital de moyenne importance et on constate en outre une mauvaise répartition

Figure 8.

\section{Distribution spatiale des formations sanitaires} selon leurs capacités d'accueil.

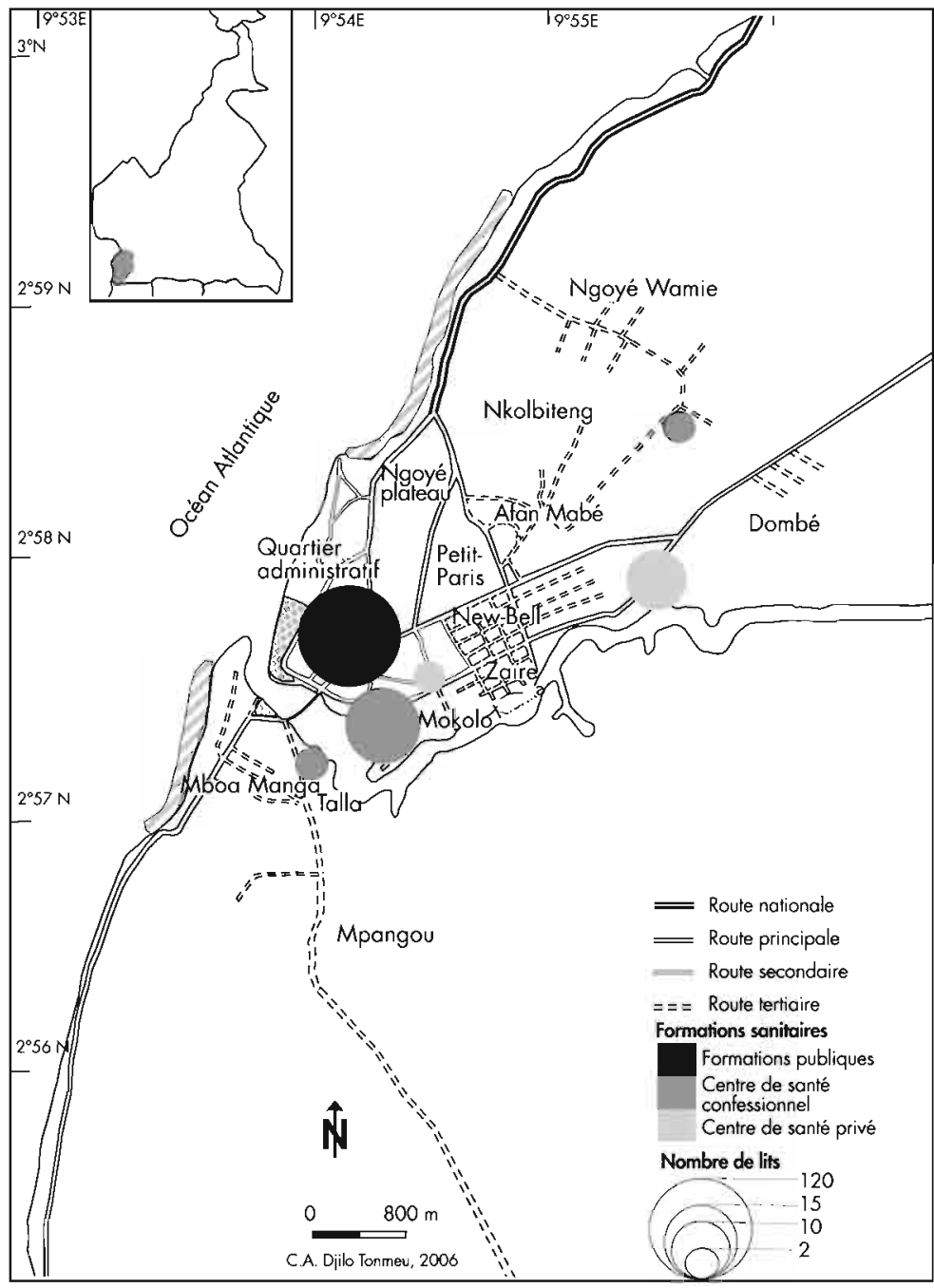


Tableau 2.

Appréciation générale du risque sanitaire par type de quartier et par type d'habitation

\begin{tabular}{|c|c|c|c|c|c|}
\hline $\begin{array}{l}\text { Type de quartier } \\
\text { et situation } \\
\text { dans la ville }\end{array}$ & $\begin{array}{l}\text { Type } \\
\text { d'habitation }\end{array}$ & $\begin{array}{l}\text { Mode d'évacuation } \\
\text { des ordures } \\
\text { ménagères }\end{array}$ & $\begin{array}{l}\text { Mode d'évacuation } \\
\text { des eaux usées } \\
\text { domestiques }\end{array}$ & $\begin{array}{l}\text { Mode d'évacuation } \\
\text { des matières fécales }\end{array}$ & $\begin{array}{l}\text { Appréciation } \\
\text { globale } \\
\text { du risque } \\
\text { sanitaire }\end{array}$ \\
\hline \multirow[t]{4}{*}{$\begin{array}{l}\text { Quartiers populaires } \\
\text { en situation centrale } \\
\text { ou péricentrale } \\
\text { (exemple Mokolo } \\
\text { ou Petit-Paris) }\end{array}$} & $\begin{array}{l}\text { Cases en matériaux } \\
\text { de récupération }\end{array}$ & $\begin{array}{l}\text { Evacuation par la mairie } \\
\text { si bonne desserte routière; } \\
\text { ordures jetées derrière } \\
\text { ou devant les cases }\end{array}$ & $\begin{array}{l}\text { Rejet dans rigoles ou } \\
\text { dans les cours d'eau }\end{array}$ & $\begin{array}{l}\text { Latrines, toilettes } \\
\text { avec canalisations } \\
\text { orientées vers les cours } \\
\text { d'eau voisins }\end{array}$ & Élevé \\
\hline & $\begin{array}{l}\text { Cases en matériaux } \\
\text { provisoires }\end{array}$ & $\begin{array}{l}\text { Évacuation par la mairie } \\
\text { si bonne desserte routière; } \\
\text { ordures jetées derrière } \\
\text { ou devant les cases }\end{array}$ & $\begin{array}{l}\text { Rejet dans rigoles ou } \\
\text { dans les cours d'eau }\end{array}$ & $\begin{array}{l}\text { Latrines, toilettes } \\
\text { avec canalisations } \\
\text { orientées vers les cours } \\
\text { d'eau voisins }\end{array}$ & Élevé \\
\hline & $\begin{array}{l}\text { Maisons modernes } \\
\text { en matériaux } \\
\text { définitifs }\end{array}$ & $\begin{array}{l}\text { Évacuation par la mairie } \\
\text { si bonne desserte routière; } \\
\text { ordures jetées derrière } \\
\text { ou devant les cases }\end{array}$ & $\begin{array}{l}\text { Rejet dans des fosses } \\
\text { septiques }\end{array}$ & $\begin{array}{l}\text { Toilettes modernes } \\
\text { avec des canalisations } \\
\text { orientées vers des fosses } \\
\text { septiques }\end{array}$ & Moyen \\
\hline & $\begin{array}{l}\text { Installations } \\
\text { hôtelières }\end{array}$ & $\begin{array}{l}\text { Ordures évacuées } \\
\text { par la municipalité }\end{array}$ & $\begin{array}{l}\text { Rejet dans des fosses } \\
\text { septiques }\end{array}$ & $\begin{array}{l}\text { Toilettes modernes } \\
\text { avec des canalisations } \\
\text { orientées vers des fosses } \\
\text { septiques }\end{array}$ & Moyen \\
\hline
\end{tabular}


Tableau 2. (suite)

Appréciation générale du risque sanitaire par type de quartier et par type d'habitation

\begin{tabular}{|c|c|c|c|c|c|}
\hline $\begin{array}{l}\text { Type de quartier } \\
\text { et situation } \\
\text { dans la ville }\end{array}$ & $\begin{array}{l}\text { Type } \\
\text { d'habitation }\end{array}$ & $\begin{array}{l}\text { Mode d'évacuation } \\
\text { des ordures } \\
\text { ménagères }\end{array}$ & $\begin{array}{l}\text { Mode d'évacuation } \\
\text { des eaux usées } \\
\text { domestiques }\end{array}$ & $\begin{array}{l}\text { Mode d'évacuation } \\
\text { des matières fécales }\end{array}$ & $\begin{array}{l}\text { Appréciation } \\
\text { globale } \\
\text { du risque } \\
\text { sanitaire }\end{array}$ \\
\hline \multirow{4}{*}{$\begin{array}{l}\text { Quartiers populaires } \\
\text { en situation } \\
\text { périphérique } \\
\text { (exemple Dombé } \\
\text { ou Talla) }\end{array}$} & $\begin{array}{l}\text { Case de type rural } \\
\text { en matériaux } \\
\text { provisoires }\end{array}$ & $\begin{array}{l}\text { Évacuation par la mairie } \\
\text { si bonne desserte routière; } \\
\text { ordures jetées derrière } \\
\text { ou devant les cases }\end{array}$ & $\begin{array}{l}\text { Rejet dans rigoles } \\
\text { ou dans les cours } \\
\text { d'eau }\end{array}$ & $\begin{array}{l}\text { Latrines; toilettes } \\
\text { avec canalisations } \\
\text { orientées vers } \\
\text { les cours d'eau voisins }\end{array}$ & Élevé \\
\hline & $\begin{array}{l}\text { Maisons } \\
\text { en matériaux } \\
\text { définitifs }\end{array}$ & $\begin{array}{l}\text { Évacuation par la mairie } \\
\text { si bonne desserte routière; } \\
\text { ordures jetées derrière } \\
\text { ou devant les cases }\end{array}$ & $\begin{array}{l}\text { Rejet dans des fosses } \\
\text { septiques }\end{array}$ & $\begin{array}{l}\text { Toilettes modernes } \\
\text { avec des canalisations } \\
\text { orientées vers des fosses } \\
\text { septiques }\end{array}$ & Moyen \\
\hline & Villa moderne & $\begin{array}{l}\text { Evacuation par la mairie } \\
\text { si bonne desserte routière; } \\
\text { ordures jetées derrière } \\
\text { ou devant les cases }\end{array}$ & $\begin{array}{l}\text { Rejet dans des fosses } \\
\text { septiques }\end{array}$ & $\begin{array}{l}\text { Toilettes modernes } \\
\text { avec des canalisations } \\
\text { orientées vers des fosses } \\
\text { septiques }\end{array}$ & Moyen \\
\hline & $\begin{array}{l}\text { Installations } \\
\text { hôtelières }\end{array}$ & $\begin{array}{l}\text { Ordures évacuées } \\
\text { par la municipalité }\end{array}$ & $\begin{array}{l}\text { Rejet dans des fosses } \\
\text { septiques }\end{array}$ & $\begin{array}{l}\text { Toilettes modernes } \\
\text { avec des canalisations } \\
\text { orientées vers des fosses } \\
\text { septiques }\end{array}$ & Moyen \\
\hline
\end{tabular}


Tableau 2. (suite)

Appréciation générale du risque sanitaire par type de quartier et par type d'habitation

\begin{tabular}{|c|c|c|c|c|c|}
\hline $\begin{array}{l}\text { Type de quartier } \\
\text { et situation } \\
\text { dans la ville }\end{array}$ & $\begin{array}{l}\text { Type } \\
\text { d'habitation }\end{array}$ & $\begin{array}{l}\text { Mode d'évacuation } \\
\text { des ordures } \\
\text { ménagères }\end{array}$ & $\begin{array}{l}\text { Mode d'évacuation } \\
\text { des eaux usées } \\
\text { domestiques }\end{array}$ & $\begin{array}{l}\text { Mode d'évacuation } \\
\text { des matières fécales }\end{array}$ & $\begin{array}{l}\text { Appréciation } \\
\text { globale } \\
\text { du risque } \\
\text { sanitaire }\end{array}$ \\
\hline \multirow[t]{3}{*}{$\begin{array}{l}\text { Quartier } \\
\text { administratif } \\
\text { en situation centrale } \\
\text { et avec une bonne } \\
\text { desserte routière }\end{array}$} & $\begin{array}{l}\text { Maison moderne } \\
\text { individuelle }\end{array}$ & $\begin{array}{l}\text { Ordures évacuées } \\
\text { par la municipalité }\end{array}$ & $\begin{array}{l}\text { Rejet dans des fosses } \\
\text { septiques }\end{array}$ & $\begin{array}{l}\text { Toilettes modernes } \\
\text { avec des canalisations } \\
\text { orientées vers } \\
\text { des fosses septiques }\end{array}$ & Moyen \\
\hline & $\begin{array}{l}\text { Immeubles } \\
\text { résidentiels }\end{array}$ & $\begin{array}{l}\text { Ordures déposées } \\
\text { dans des seaux } \\
\text { et bacs à ordures } \\
\text { et évacuées } \\
\text { par la municipalité; } \\
\text { ordures rejetées } \\
\text { à travers les fenêtres }\end{array}$ & $\begin{array}{l}\text { Rejet dans des fosses } \\
\text { septiques ou } \\
\text { à travers les claustras } \\
\text { des immeubles }\end{array}$ & $\begin{array}{l}\text { Toilettes modernes } \\
\text { avec des canalisations } \\
\text { orientées vers des fosses } \\
\text { septiques }\end{array}$ & Moyen \\
\hline & $\begin{array}{l}\text { Bâtiments } \\
\text { administratifs }\end{array}$ & $\begin{array}{l}\text { Ordures évacuées } \\
\text { par la municipalité }\end{array}$ & $\begin{array}{l}\text { Rejet dans des fosses } \\
\text { septiques }\end{array}$ & $\begin{array}{l}\text { Toilettes modernes } \\
\text { avec des canalisations } \\
\text { orientées vers des fosses } \\
\text { septiques }\end{array}$ & Moyen \\
\hline
\end{tabular}


Tableau 2. (suite)

Appréciation générale du risque sanitaire par type de quartier et par type d'habitation

\begin{tabular}{|c|c|c|c|c|c|}
\hline $\begin{array}{l}\text { Type de quartier } \\
\text { et situation } \\
\text { dans la ville }\end{array}$ & $\begin{array}{l}\text { Type } \\
\text { d'habitation }\end{array}$ & $\begin{array}{l}\text { Mode d'évacuation } \\
\text { des ordures } \\
\text { ménagères }\end{array}$ & $\begin{array}{l}\text { Mode d'évacuation } \\
\text { des eaux usées } \\
\text { domestiques }\end{array}$ & $\begin{array}{l}\text { Mode d'évacuation } \\
\text { des matières fécales }\end{array}$ & $\begin{array}{l}\text { Appréciation } \\
\text { globale } \\
\text { du risque } \\
\text { sanitaire }\end{array}$ \\
\hline \multirow{6}{*}{$\begin{array}{l}\text { Front de mer } \\
\text { (exemple } \\
\text { partie ouest } \\
\text { de Mboa Manga } \\
\text { ou Ngoye Plateau) }\end{array}$} & $\begin{array}{l}\text { Hangars } \\
\text { et étals } \\
\text { commerciaux }\end{array}$ & $\begin{array}{l}\text { Entassement devant } \\
\text { les hangars et étals } \\
\text { ou dans des poubelles }\end{array}$ & $\begin{array}{l}\text { Déversement dans } \\
\text { des rigoles séparant } \\
\text { les hangars et étals }\end{array}$ & $\begin{array}{l}\text { Utilisation par } \\
\text { les commerçants } \\
\text { et clients des latrines } \\
\text { des maisons voisines }\end{array}$ & Très élevé \\
\hline & $\begin{array}{l}\text { Boutiques } \\
\text { et magasins }\end{array}$ & $\begin{array}{l}\text { Entassement devant } \\
\text { les hangars et étals } \\
\text { ou dans des poubelles }\end{array}$ & $\begin{array}{l}\text { Déversement dans } \\
\text { des rigoles séparant } \\
\text { les hangars et étals }\end{array}$ & $\begin{array}{l}\text { Utilisation par } \\
\text { les commerçants } \\
\text { et clients des latrines } \\
\text { des maisons voisines }\end{array}$ & Très élevé \\
\hline & $\begin{array}{l}\text { Cases } \\
\text { de type rural }\end{array}$ & $\begin{array}{l}\text { Evacuation par la mairie } \\
\text { si bonne desserte routière; } \\
\text { ordures jetées derrière } \\
\text { ou devant les cases }\end{array}$ & $\begin{array}{l}\text { Rejet dans rigoles } \\
\text { ou dans les cours } \\
\text { d'eau }\end{array}$ & $\begin{array}{l}\text { Latrines, } \\
\text { toilettes avec canalisations } \\
\text { orientées vers l'océan }\end{array}$ & Élevé \\
\hline & $\begin{array}{l}\text { Maisons } \\
\text { en matériaux } \\
\text { provisoires }\end{array}$ & $\begin{array}{l}\text { Évacuation par la mairie } \\
\text { si bonne desserte routière; } \\
\text { ordures jetées derrière } \\
\text { ou devant les cases }\end{array}$ & $\begin{array}{l}\text { Rejet dans les rigoles } \\
\text { ou dans l'océan }\end{array}$ & $\begin{array}{l}\text { Latrines, } \\
\text { toilettes avec canalisations } \\
\text { orientées vers l'océan }\end{array}$ & Élevé \\
\hline & $\begin{array}{l}\text { Maisons modernes } \\
\text { individuelles }\end{array}$ & $\begin{array}{l}\text { Ordures évacuées } \\
\text { par la municipalité }\end{array}$ & $\begin{array}{l}\text { Rejet dans des fosses } \\
\text { septiques }\end{array}$ & $\begin{array}{l}\text { Toilettes modernes avec } \\
\text { des canalisations orientées } \\
\text { vers des fosses septiques }\end{array}$ & Moyen \\
\hline & $\begin{array}{l}\text { Installations } \\
\text { hôtelières }\end{array}$ & $\begin{array}{l}\text { Ordures évacuées } \\
\text { par la municipalité }\end{array}$ & $\begin{array}{l}\text { Rejet dans des fosses } \\
\text { septiques }\end{array}$ & $\begin{array}{l}\text { Toilettes modernes avec } \\
\text { des canalisations orientées } \\
\text { vers des fosses septiques }\end{array}$ & Moyen \\
\hline
\end{tabular}

Source: enquêtes et observations de terrain, mai 2006 
spatiale de ces équipements. Le centre administratif concentre à lui tout seul l'essentiel du personnel soignant. La ville compte au total 68 professionnels de santé (médecins, infirmiers, aides-soignants, techniciens de laboratoires d'analyses médicales), pour une population estimée à plus de 40000 habitants.

C'est dans les quartiers où on enregistre de fortes densités de population (Newton, Afan Mabé, Mboa Manga) que, paradoxalement, il n'existe pas de centre de santé. Le centre de santé d'Afan Mabé et celui des sœurs catholiques ne disposent chacun que de deux lits d'hôpital, ce qui réduit les soins aux consultations. Les malades se font examinés et, s'il y a nécessité d'hospitalisation, ils sont obligés d'aller à l'hôpital de district ou dans d'autres centres pouvant les accueillir. La capacité d'accueil de l'ensemble des formations sanitaires de la ville est de 160 lits, soit un ratio d'un lit pour 250 habitants, phénomène d'autant plus problématique que les populations des localités voisines viennent généralement se soigner à Kribi. Au regard de cette situation, il est clair que la ville n'est pas suffisamment armée pour faire face à une forte épidémie de choléra ou de typhoide.

Le tableau 2 donne un aperçu général du risque sanitaire à Kribi, sur la base du binôme constitué par les ordures ménagères et les eaux usées.

\section{La prévention sanitaire par une gestion rationnelle des ordures ménagères et des eaux usées}

Une possible amélioration dans la gestion des ordures ménagères et des eaux usées pour minimiser le risque sanitaire, en l'absence d'un système d'assainissement qui prendrait encore plusieurs années à être mise en place, serait d'appliquer la réglementation existante en matière d'hygiène et de salubrité, de prendre en compte la configuration physique du site urbain de Kribi en vue d'une meilleure gestion de l'écoulement des eaux de surface et de sensibiliser les populations. 


\section{Aspects juridiques et réglementaires}

Contrairement à ce que laisserait croire la situation de bien des villes du Cameroun, les gouvernements successifs ont beaucoup légiféré en matière urbanistique et environnementale. Dans une étude publiée en 1999, R. J. AssAKO AssakO a effectué un inventaire, montrant que, de la période coloniale à nos jours, le Cameroun s'est doté d'un important arsenal d'ordonnances, de lois, de décrets et d'arrêtés pour réglementer l'occupation du sol en milieu urbain et la protection de l'environnement, l'hygiène et le cadre de vie.

En ce qui concerne la gestion de l'eau, les textes ci-après peuvent valablement être évoqués: la loi $\mathrm{n}^{\circ}$ 98/005 du 14 avril 1998, portant régime de l'eau, fixe les conditions d'exploitation et d'utilisation rationnelle de l'eau au Cameroun; et le décret n²005/3089/PM du 29 août 2005 portant précision des règles d'assiette, de recouvrement et de contrôle de la taxe d'assainissement et de la redevance de prélèvement des eaux.

Au titre de cette loi, sont interdits:

- tout déversement, écoulement, rejet, enfouissement direct ou indirect dans les eaux de toute matière solide, liquide, ou gazeuse susceptible d'altérer la qualité des eaux de surface ou souterraines ou des mers;

- le nettoyage et l'entretien des véhicules à moteur, des machines à combustion interne et autres engins similaires à proximité des eaux.

De même, est punie d'un emprisonnement de cinq à quinze ans et d'une amende de dix à vingt millions, toute personne qui altère la qualité des eaux.

Il ressort de cette situation que ce n'est pas l'absence de législations en matière de conservation et de préservation de l'environnement qui pose problème, mais les incohérences, les fragmentations de celles-ci qui rendent leur application extrêmement difficile. À titre d'exemple et comme le disent si bien P. A. LeKEANYl et al. (2000), il n'existe pas de structure spécifique définissant la politique d'assainissement c'est-à-dire, conception, réalisation, gestion et 
entretien d'ouvrages, normes de rejets, etc. À cet effet, il est important de réorienter et de mieux définir cette législation en prenant en compte les représentations et les comportements individuels ou de groupes (ASSAKO AsSAKO et al., 2005).

L'approvisionnement en eau potable et l'assainissement des eaux usées dans les pays en développement constituent l'une des priorités internationales énoncées dans les Objectifs du millénaire, adoptés par l'assemblée générale des Nations unies. Ces objectifs visent la réduction de moitié, d'ici 2015 , du pourcentage de la population qui n'a pas accès de façon durable à de l'eau potable et à des services d'assainissement des eaux de base. Cependant, l'un des obstacles à l'application de certains grands projets dans les pays en développement est, au-delà de l'inapplicabilité des réglementations existantes, l'ignorance des populations face aux dangers que représentent certaines pratiques sur leur environnement ou sur leur santé.

\section{Sensibilisation et relance des comités d'hygiène et de salubrité}

La mise en œuvre des politiques efficaces de gestion des déchets passe par la volonté politique et l'éducation des populations sur les risques que ces déchets représentent pour leur santé. Car, dans nos villes, par rapport à l'eau, l'assainissement n'est pas ressenti par les populations comme un besoin immédiat (sauf en ce qui concerne l'évacuation des eaux de pluies au moment d'inondations). Il s'agirait dans ce cas d'organiser des séances d'éducation sanitaires dans les quartiers et même dans les différentes formations sanitaires de la ville. À cet effet, les maladies dont les germes se développent dans des milieux insalubres devraient être au centre de ces journées d'éducation. Les quartiers Afan Mabé, Mokolo, Dombé qui se caractérisent par une grande promiscuité et où on enregistre de nombreux cas de maladies devraient faire l'objet d'une attention particulière. Cet état de chose pourrait aboutir à la mise sur pied des comités d'hygiène et de salubrité.

Il s'agit ici d'impliquer les populations dans le processus d'assainissement de leur cadre de vie, à travers l'enlèvement des déchets domestiques aux alentours des maisons d'habitations. Il est à 
noter que plusieurs actions ont déjà été entreprises par la municipalité de Kribi pour gérer les problèmes d'insalubrité constatée dans la ville. On peut citer pour exemple le «Jeudi propre», qui était une opération de nettoyage de la ville par les populations tous les jeudis matin. Dans le même sens, des comités d'hygiène recrutés parmi les populations avaient été installés dans les quartiers et sous la supervision de la municipalité. Cependant, toutes ces actions n'ont duré que le temps de leur création et sur le terrain rien de concret n'est fait. La relance de ces comités devrait s'accompagner de dotation en matériels (pelles, houes, brouettes, machettes...), permettant aux populations de ramasser elles-mêmes les ordures dans les quartiers inaccessibles. De même, des bacs à ordures devraient être installés dans les quartiers, les carrefours et dans le centre commercial, et ceux-ci devraient être vidés systématiquement par les agents de ramassage de la municipalité.

\section{Intensification du ramassage des ordures ménagères et création d'une station d'épuration des eaux usées}

Le service de collecte d'ordures ménagères dont dispose la commune souffre de sous-équipement et le personnel impliqué dans ce travail est insuffisant. Il serait important de renforcer le matériel disponible en augmentant le nombre de camions, d'outils de ramassage et les agents de ramassage. La décharge municipale qui présente de grands risques sanitaires devrait être systématiquement traitée avec des substances qui réduisent l'effet nocif des déchets sur les cours d'eau environnants et même sur le sol.

Il est tout aussi urgent dans cette ville de développer des réseaux de collectes d'eaux usées et de créer une station d'épuration pour le traitement des effluents dans les zones commerciale, administrative et dans le couloir hôtelier sur emprise maritime. Dans les quartiers non structurés où les toilettes traditionnelles sont mal aménagées, on pourrait envisager un service de désinfection de ces latrines au moins une fois par mois. 


\section{Conclusion}

Cette étude visait à montrer comment la mauvaise gestion des eaux usées et des déchets domestiques à Kribi engendre de nombreux risques sanitaires qui contribuent à fragiliser les conditions de vie déjà précaires des populations. Cette situation de menace permanente est la résultante de plusieurs pratiques sociales et spatiales non soucieuses de l'entretien et du respect de l'environnement de vie. De même, la configuration du site et l'inexistence d'un système d'assainissement fiable et adapté se présentent comme des facteurs d'aggravation. À cet effet, la nécessité de mettre en place des techniques et outils d'assainissement respectant la configuration géomorphologique du site et les pratiques culturelles s'impose dans cette ville où la majorité de la population est issue des zones rurales défavorisées. Cependant, un effort de respect des règles d'hygiène et l'entretien du cadre de vie sont attendus de la part des populations. Ce dernier aspect met en évidence la nécessité d'une approche croisée entre anthropologues et géographes pour mieux décrypter le processus de perception et de gestion des interfaces environnement/santé.

\section{Références bibliographiques}

AMAT-ROzE J.-M., 2000 - Santé et tropicalité en Afrique subsaharienne: un système multirisques. Afrique Contemporaine, $\mathrm{n}^{\circ} 195$, juillet.

ASSAKO ASSAKO R. J., 1999b - Critique de la politique urbaine au Cameroun: instruments, résultat et évolution. Revue de Géographie du Cameroun, $\mathrm{n}^{\circ} 1 / 1999,33-67$.

ASSAKO ASSAKO R. J., 2001 a - Formulation et validation d'une hypothèse de pollution de l'eau de surface: le cas du lac municipal de Yaoundé. Déchets, Science et Techniques, Insa de Lyon.

ASSAKO ASSAKO R. J., 2001 b - «Réflexions sur le processus de création et de développement des villes au Cameroun ". H\&A; Afrique Monde Noir; Diaspora Noire, Éditions L'Harmattan, $\mathrm{n}^{\circ} 1$.

Assako Assako R. J., 2005 - «Problématique de l'estimation de la qualité de vie dans un front d'urbanisation en Afrique: le cas du Bois des Singes à Douala (Cameroun)». In Bley D. (éd.): Cadre de vie et travail: les dimensions d'une qualité de vie au quotidien, Éditions Edisud : 65-85. 
Assako R. J., MeVA'A ABOMo D., TChuikoua L. B., 2005 - «Étude géographique de l'épidémie de choléra à Douala ou la qualité de vie à l'épreuve des pratiques urbaines $»$. In Fleuret S. (dir.): Espaces, qualité de vie et bien-être, Presses Universitaires d'Angers, Angers: 307-315.

ASSAKo Assako R. J., 2006 - «Les épidémies de choléra à Douala et au Cameroun: entre la pauvreté urbaine et la défaillance du système de gestion de l'environnement urbain $»$. In Dorier-Apprill E. (éd.): Ville et Environnement, Paris, Éditions Sedes: 365-374.

Assako Assako R. J., Bley D., Simard F., 2006 - Apports des sciences sociales et de l'entomologie dans l'analyse de l'endémicité du paludisme à Hévécam, une agro-industrie du Sud-Cameroun. Revue Géo-Éco-Trop.

DaGorne A., Dars R., 1999 - Les risques naturels. La cindynique. Paris, PUF, Coll. «Que sais-je? », n³533, 128 p.

Djilo Tonmeu C. A., 2006 - Cartographie sanitaire de la ville de Kribi: application au paludisme. Mémoire de maîtrise de géographie, département de Géographie, faculté des Lettres et Sciences humaines de l'université de Douala, $121 \mathrm{p}$.

DORIER-APPRILl E., 2006 - « La gestion de l'eau et des déchets dans les villes des PVD, entre modèles exogènes et pratiques populaires". In Dorier-Apprill E. (éd.): Ville et Environnement, Paris, Éditions Sedes : 385-412.

LAVERGNe C., GABERT J., 2005 - Monter un projet d'assainissement dans les quartiers urbains pauvres des pays en développement: une autre approche. Pistes et réflexions pour les monteurs de projet. http://perso.wanadoo.fr/experians/experians/assainissement.html

LeKeAnyl Agendia P., Fonkou T., SONWA J. D., Mefenya R., Kengne Y. M., Zambo Amougou J. M., 2000 -Collecte, épuration et évacuation des eaux usées dans les lotissements SIC-MAETUR de Yaoundé. Revue de Géographie du Cameroun, vol. XIV, n² 2: 173-192.

Meva'A Aвомо D., 2006 - Étude géographique de l'endémicité du paludisme dans l'interface environnement et santé à Kribi. Mémoire de DEA, département de Géographie de la faculté des Lettres et Sciences humaines de l'université de Douala, 163 p.

PÉCAUD D., 2005 - «Dangers, risques et précautions: quels leviers pour les politiques de prévention?". In Bley D. (éd.): Cadre de vie et travail: les dimensions d'une qualité de vie au quotidien, Aix-en-Provence, Edisud: 265-279. 
Annexe

Quelques germes pathogènes liés aux eaux usées et maladies engendrées, avec modes de transmission

\begin{tabular}{|c|c|c|c|}
\hline Groupe & Germes ou parasites & Affection & Mode de transmission \\
\hline Bactéries & $\begin{array}{l}\text { Salmonella spp. } \\
\text { Salmonella typhi } \\
\text { et S. paratyphoid } \\
\text { Campylobacter spp. } \\
\text { Escherichia coli } \\
\text { Vibrio cholerae } \\
\text { Leptospira spp. } \\
\text { Clostridium } \\
\text { Brucella tularensis }\end{array}$ & $\begin{array}{l}\text { Entérites, diarrhée, campylobactériose } \\
\text { Dysenterie bactérienne } \\
\text { Choléra, entérite } \\
\text { Leptospirose } \\
\text { Tétanos, gangrène } \\
\text { Tularémie }\end{array}$ & \multirow[t]{2}{*}{ Ingestion d'aliments et d'eau souillés } \\
\hline \multirow[t]{3}{*}{ Virus } & $\begin{array}{l}\text { Polyvirus } \\
\text { Coxsackievirus A } \\
\text { Coxsackievirus B } \\
\text { Echovirus }\end{array}$ & $\begin{array}{l}\text { Poliomyélite, entérites } \\
\text { Courbatures musculaires } \\
\text { Méningite } \\
\text { Diarrhées, hépatites }\end{array}$ & \\
\hline & $\begin{array}{l}\text { Adenovirus } \\
\text { Reovirus } \\
\end{array}$ & $\begin{array}{l}\text { Infections respiratoires, attaque du système nerveux } \\
\text { Infections respiratoires, diarrhées, hépatite }\end{array}$ & $\begin{array}{l}\text { Respiration des vapeurs } \\
\text { provenant de sources d'eau souillées }\end{array}$ \\
\hline & Hépatite A & Hépatite infectieuse & \multirow[t]{3}{*}{ Ingestion d'aliments et d'eaux infectés } \\
\hline Protozoaires & $\begin{array}{l}\text { Entamoeba histolitica } \\
\text { Gardia intestinalis } \\
\text { Balantidium } \\
\text { Giardia lamblia }\end{array}$ & $\begin{array}{l}\text { Dysenterie amibienne } \\
\text { Gardiase } \\
\text { Balantidiase } \\
\text { Lambliase }\end{array}$ & \\
\hline Helminthes & $\begin{array}{l}\text { Ascaris lumbricoides } \\
\text { Ancylostoma doudenale } \\
\text { Taenia spp. }\end{array}$ & $\begin{array}{l}\text { Ascaridiase } \\
\text { Ankylostomiase } \\
\text { Ténia }\end{array}$ & \\
\hline & $\begin{array}{l}\text { Schistosoma } \\
\text { Sarcoptes scabiei }\end{array}$ & $\begin{array}{l}\text { Schistosomiase ou bilharziose } \\
\text { Gale }\end{array}$ & $\begin{array}{l}\text { Contact corporel avec de l'eau } \\
\text { contaminée (pénétration cutanée) }\end{array}$ \\
\hline
\end{tabular}

D'apres diverses sources dont LEKEANYI et al. (2000). 



\section{Réflexions}

\section{sur les obstacles}

\section{à une prévention efficace de la maladie du sommeil en Guinée maritime}

\section{Jean-Pierre HERVOUËT \\ Moïse KAGBADOUNO \\ Mamadou CAMARA}

Depuis 1997, la lutte contre la maladie du sommeil a repris, activement, sur le littoral guinéen grâce à une collaboration entre le ministère de la Santé (division Prévention, section Maladies transmissibles, puis PNLTHA - Programme national de lutte contre la trypanosomiase humaine - à partir de 2002), l'institut Pierre-Richet, l'IRD et bien sûr l'OMS, auxquels s'est associé, depuis 2005, le Cirdes de Bobo Dioulasso. Cependant, malgré des prospections menées en 1997, 1998, 2002, 2003, 2004, 2005 et ayant touché 48 villages, pour la seule préfecture de Dubreka, on n'a constaté aucune modification significative du pourcentage de nouveaux malades ${ }^{1}$ dépistés dans les villages ayant bénéficié d'au moins quatre passages des équipes de lutte contre la maladie: de 1997 à 2006, ce pourcentage a oscillé entre $1,32 \%$ et $1,03 \%$, en passant par 1,58 en 1998 et 0,75 en $2005\left(X^{2}=0,22\right)$. 
Si l'analyse de l'activité des services de contrôle de la maladie ${ }^{2}$ permet d'apporter des éléments de réponses à cette situation préoccupante, elle permet surtout de poser, très concrètement, le problème de la simplification des protocoles des dépistages actifs et des conséquences épidémiologiques et stratégiques qui en découlent.

\section{La trypanosomiase humaine africaine (THA) : une vieille connaissance de la Guinée maritime}

La présence de la maladie du sommeil fut signalée dès 1909 dans les cercles de Boffa et de Forécariah puis, au début des années 1920, plusieurs cas furent traités à Conakry (JAMOT, 1933). Le silence se fit alors sur cette endémie dans l'ensemble des rapports médicaux disponibles. Pourtant, la maladie du sommeil était bien présente en zone littorale guinéenne comme l'atteste la création, dès 1939, du Secteur annexe n 34 de Boké par G. Muraz, puis, en 1943 du SA 72 suite à la découverte d'une infection importante de la banlieue de Conakry (canton de Kaporo) et des îles de Los toutes proches. Ce secteur, en raison de l'ampleur de la maladie découverte dans les cantons du littoral proches du chef-lieu de la colonie, fut, en 1947, transformé en Secteur spécial, installé à Dubreka et qui coiffa, un peu plus tard, les circonscriptions de Forécariah, Coyah, Conakry, Dubreka, Boffa et Boké. Malgré son importance, la maladie y était cependant loin de faire les ravages qu'elle occasionnait en MoyenneGuinée (Labé, Pita, Mamou) ou en Guinée forestière (Kissidougou, Guékédou, N'zérékoré, Macenta...)

Selon les rapports des médecins chefs des secteurs de Guinée ${ }^{3}$, fin 1957, juste avant l'indépendance de cette colonie française,

2 Qui s'appuie sur les expériences acquises par le travail pluridisciplinaire réalisé dans les foyers de Côte d'Ivoire depuis 1979 et ayant contribué au contrôle de la maladie dans le foyer de Vavoua.

3 Consultés à Bobo Dioulasso au Centre Muraz, en 1979 et à l'OOAS (Organisation ouest-africaine de la santé) en 2005. 
Figure 1.

Localisation et noms cités.

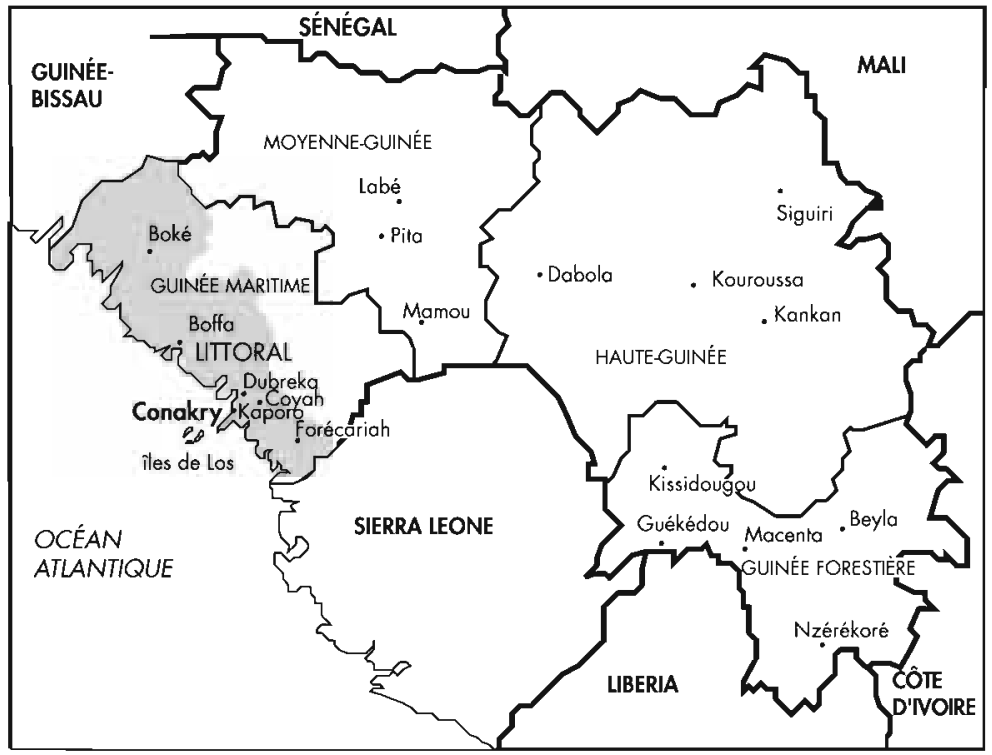

2667 trypanosomés issus du littoral avaient été soignés depuis 1939 sur les 95137 traités pour l'ensemble du territoire du nouvel État, soit moins de $3 \%$ du total. Ce pourcentage était cependant en constante augmentation et atteignait $12 \%$ en 1957, ce qui est plus la conséquence de la maîtrise de la maladie sur le reste du territoire que de l'augmentation du nombre de cas dépistés par les secteurs 34 (Boké), 35 (Boffa) et 72 (Conakry puis Dubreka).

En 1962, pour la Guinée, l'OMS jugeait la situation bien en main, puisque le taux de fréquence annuelle était de 0,065\% en 1960 (sur 1650334 sujets examinés) et de 0,066\% en 1961 (sur 1304879 sujets examinés) (OMS, 1962). Ces mêmes années, il était dépisté, respectivement, 44 et 35 malades dans l'actuelle préfecture de Dubreka et en 1962, dans ce même espace, étaient dépistés en prospection, 59 malades sur 51517 personnes visitées $(0,11 \%)$ et au total, $105 \mathrm{NT}^{4}$ sur 84805 habitants $(0,12 \%$ ) (rapport du Secteur spécial $\left.\mathrm{n}^{\circ} 1,1962\right)$. 
Il est ensuite très difficile de connaître l'évolution des activités des secteurs, ces derniers ayant été redécoupés et leurs archives introuvables. En 1979, l'OMS considère que la situation reste stable grâce à une surveillance médicale et une chimioprophylaxie régulière (OMS, 1979). À peine plus tard, l'Organisation mondiale de la santé constate que l'on ne dispose d'aucun renseignement sur la situation épidémiologique récente en Guinée et que les cas récemment notifiés (...) témoignent de la poursuite de la transmission (OMS, 1986) tout en sous-estimant l'ampleur de la maladie en Guinée. En effet, les cas rapportés par l'organisation internationale sont souvent inférieurs, pour l'ensemble de la Guinée, à ceux connus pour la seule hypnoserie de Dubreka grâce à un registre de traitement des malades conservé à Makompo, le centre de traitement ( 3 NT pour l'OMS en 1975 contre 32 soignés à Dubreka; 59 en 1982 contre 68).

Deux centres de traitement des trypanosomés, au moins, restèrent actifs en zone littorale: celui de Dubreka renommé Secteur spécial $\mathrm{n}^{\circ} 1$ à l'indépendance du pays et celui de Madina (Conakry) qui, en 1976, organisait encore des prospections sur les îles de Los. Malheureusement, on n'a retrouvé que les données du secteur de Dubreka pour les années 1960, 1961 et 1962, puis pour les années postérieures à 1970. Comme les registres sauvegardés ne mentionnent que les malades traités à Dubreka et pas ceux soignés ailleurs (dont Madina), les informations dont nous disposons sont inférieures à la réalité.

D'après les données disponibles, de 1970 à 1990, 35 malades originaires du littoral continuèrent à être soignés annuellement, soit un peu plus de $40 \%$ du total guinéen "affiché ". Cette moyenne tomba à un peu moins de 30 pour la période 1991-1995, ce qui représentait, déjà, plus de $75 \%$ du total des trypanosomés traités connus en Guinée.

Mais aujourd'hui, les malades soignés originaires de la zone littorale représentent l'écrasante majorité de tous les trypanosomés soignés en Guinée: 721 soignés à Dubreka depuis 1995, pour une dizaine seulement pour le reste de la Guinée. Ces chiffres ne signifient malheureusement pas que la maladie a disparu des autres régions, mais simplement que l'essentiel des opérations de dépistages se déroule dans la banlieue de la capitale et les préfectures les plus 
proches. Ici, comme ailleurs, la maladie, reconnue, est présente là où on la cherche (HERvouËT et al., 2000) et il ne faut pas confondre absence d'informations et de données sur les malades et absence réelle de la maladie.

\section{La THA contemporaine: une distribution très hétérogène}

Historiquement, la distribution de la maladie était hétérogène et elle le demeure aujourd'hui quelle que soit l'échelle considérée: régionale, départementale ou locale. C'est ce qu'ont montré les sept prospections menées, depuis 1997, par le Programme national de lutte contre la trypanosomiase humaine africaine en zone littorale guinéenne et comme le montrent aussi les données d'archives disponibles.

Tableau 1.

Évolution de la THA par région en Guinée (1934-1955)

Nombre de NT dépistés

\begin{tabular}{|lcccc}
\hline & 1934-1938 & 1939-1945 & 1946-1950 & 1951-1955 \\
\hline Moyenne-Guinée & 8890 & 24298 & 9883 & 5374 \\
\hline Guinée forestière & 18786 & 24946 & 12634 & 2323 \\
\hline Haute-Guinée & 1 & 6059 & 1492 & 1593 \\
\hline Guinée maritime & 4 & 758 & 1025 & 757 \\
\hline
\end{tabular}

Sources: JAMOT, 1935: Annales de médecine et de pharmacie coloniale, 1935-1939;

Rapports annuels du SGAMS et du SGHMP, 1939-1957; Rapport de la mission CCTA, 1964.

Nous l'avons dit, depuis 10 ans, la plus grande partie des sommeilleux soignés en Guinée est originaire de Guinée maritime, les malades issus de l'intérieur étant très minoritaires. C'est dans cette Guinée littorale, qu'historiquement, le déclin de la maladie a été le plus lent, jusqu'à placer cette région largement en tête des zones infectées. 
À l'échelle de la Guinée maritime, cette hétérogénéité de la distribution des malades se retrouve quelles que soient les périodes considérées: en 2004-2005, comme avant l'indépendance, non seulement les préfectures, mais aussi les "cantons" sont très diversement affectés, comme l'indiquent les tableaux 2, 3 et 4 .

Tableau 2.

Origine cantonale des trypanosomés dépistés en Guinée littorale de 1942 à 1962

\begin{tabular}{|llrccc|}
\hline Cercle & Canton & 1942-1946 & 1947-1952 & 1953-1957 & $\mathbf{1 9 6 2}$ \\
\hline Conakry & Los & 50 & 11 & 0 & $?$ \\
& Kaporo & 73 & 54 & 11 & $?$ \\
& Ville & 9 & 20 & 0 & $?$ \\
& Total & 132 & 85 & 11 & $?$ \\
\hline Coyah & Tous & 21 & 26 & 3 & 6 \\
\hline Dubreka & Bacondji & 0 & 1 & 4 & 0 \\
& Bramaya & 75 & 43 & 5 & 19 \\
& Garandji & 0 & 0 & 0 & 0 \\
& Kabitaye & 127 & 33 & 40 & 22 \\
& Labaya & 0 & 3 & 0 & 0 \\
& Tabounsou & 176 & 34 & 11 & 12 \\
& Total & 378 & 114 & 60 & 53 \\
\hline Forékariah & Tous & 0 & 2 & 23 & $?$ \\
\hline Boffa & Tous & & 84 & 40 & $?$ \\
\hline Boké & Tous & 0 & 0 & 5 & $?$ \\
\hline
\end{tabular}

$?$ = absence de données.

Sources: Rapports annuels Secteur 72, 1943-1957.

Secteur 34: Rapport annuel Secteur spécial n²1, 1962.

Tableau 3.

Prévalence de la THA en Guinée maritime en 2004-2005, par préfecture

\begin{tabular}{|lccc|}
\hline & Population vue & NT & Prévalence \\
\hline Préfecture de Dubreka & 5341 & 37 & $0,69 \%$ \\
Préfecture de Boffa & 7879 & 24 & $0,30 \%$ \\
Préfecture de Forécariah & 7522 & 18 & $0,24 \%$ \\
Préfecture de Boké & 1151 & 0 & $0,00 \%$ \\
\hline
\end{tabular}

Source: Rapports PNLTHA. 
Tableau 4.

Origine cantonale des trypanosomés traités à Dubreka depuis 1970

\begin{tabular}{|c|c|c|c|c|c|}
\hline \multicolumn{6}{|c|}{ Guinée littorale } \\
\hline Préfecture & Canton & $1970-1979$ & $1980-1989$ & 1990-1999 & 2000-2005 \\
\hline \multirow[t]{4}{*}{ Boffa } & Koba & 13 & 10 & 14 & 33 \\
\hline & Thia & 1 & 10 & 83 & 85 \\
\hline & Autres & 4 & 1 & 5 & 10 \\
\hline & Total & 18 & 21 & 102 & 128 \\
\hline Boké & Tous & 3 & 2 & 6 & 3 \\
\hline \multirow[t]{4}{*}{ Conakry } & Los & 7 & 2 & 0 & 0 \\
\hline & Kaporo & 30 & 35 & 29 & 15 \\
\hline & Ville & 12 & 11 & 3 & 2 \\
\hline & Total & 49 & 48 & 32 & 17 \\
\hline Coyah & Tous & 50 & 28 & 12 & 1 \\
\hline \multirow[t]{7}{*}{ Dubreka } & Bacondji & 3 & 1 & 0 & 0 \\
\hline & Bramaya & 57 & 30 & 46 & 76 \\
\hline & Garandji & 2 & 0 & 0 & 0 \\
\hline & Kabitaye & 167 & 163 & 166 & 97 \\
\hline & Labaya & 2 & 1 & 0 & 0 \\
\hline & Tabounsou & 155 & 71 & 49 & 27 \\
\hline & Total & 386 & 266 & 261 & 200 \\
\hline Forékariah & Tous & 39 & 73 & 74 & 47 \\
\hline \multicolumn{6}{|c|}{ Guinée intérieure } \\
\hline Préfecture & Canton & $1970-1979$ & $1980-1989$ & 1990-1999 & $2000-2005$ \\
\hline Dalaba & Tous & 1 & & & \\
\hline Faranah & Tous & 1 & & & \\
\hline Guékédou & Tous & & & & 3 \\
\hline Kindia & Tous & & & 1 & 1 \\
\hline Kissidougou & Tous & 2 & & & \\
\hline Kouroussa & Tous & & 1 & 1 & \\
\hline Macenta & Tous & & & & 1 \\
\hline Mamou & Tous & 1 & & 1 & \\
\hline Pita & Tous & 2 & & 1 & \\
\hline Siguiri & Tous & 1 & & & 2 \\
\hline Télimélé & Tous & 3 & & & 1 \\
\hline \multicolumn{6}{|l|}{ Étranger } \\
\hline Sénégal & & 1 & & & \\
\hline
\end{tabular}

Source: Référencier Dubreka. 
Lactuelle préfecture de Dubreka regroupe six anciens cantons, et les trois cantons qui étaient sérieusement touchés par la maladie du sommeil durant la période coloniale le demeurent aujourd'hui: ce sont les cantons de Tabounsou (Dubreka), du Kabitaye (Khorira) et du Bramaya (Wassou-Tanéné) (fig. 2). Ces trois cantons sont

Figure 2.

\section{Dubreka:}

milieux et suspects sérologiques en 1997.

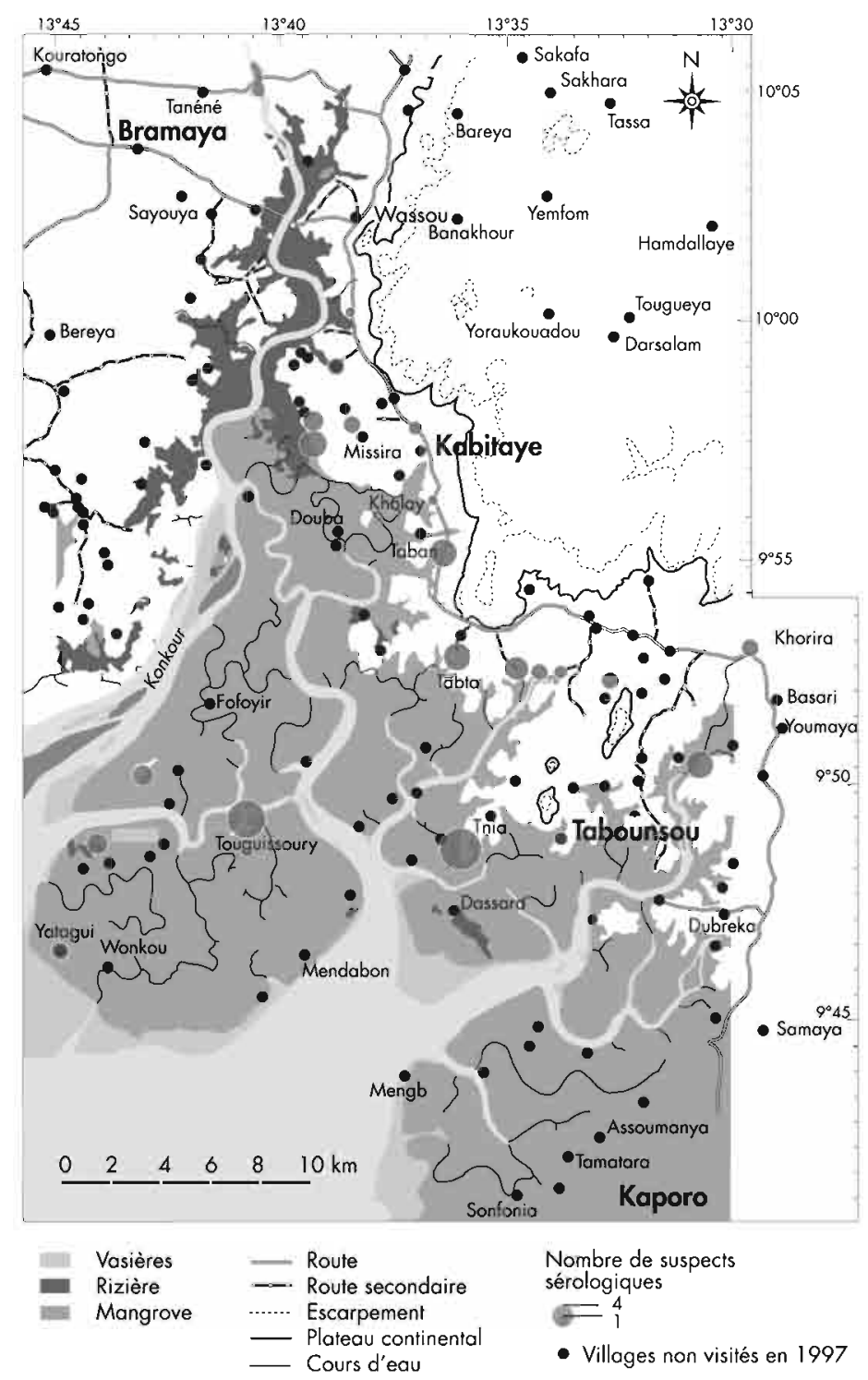


situés en bordure du golfe de Guinée et débordent largement sur la mangrove. Ils jouxtent le canton de Kaporo, banlieue de Conakry, au sud-ouest, mais aussi les cantons de Koba et de Thia, les plus atteints du secteur annexe de Boffa, plus au nord et s'étendant, en partie, comme les autres cantons cités ci-dessus, sur des zones de mangrove. Les trois autres cantons de Dubreka, comme les cantons peu touchés de Boffa, n'ont pas d'accès à la mer et leur économie est plus tournée vers les contreforts du Fouta Djalon.

Les disparités géographiques constatées dans la distribution de la maladie au cours de la période coloniale restent les mêmes de nos jours, comme si, dans ces zones indemnes d'immigration d'allogènes, existait un réel génie épidémiologique de la THA à l'emplacement des foyers historiques (PENCHENIER et al., 1996).

Ces disparités profondes se retrouvent au niveau des villages, comme le montrent les résultats des prospections menées en 1997 dans un environnement complexe (fig. 2 et tabl. 5).

Rappelons, en outre, que ce sont les villages du plateau littoral utilisant la mangrove et ceux du système fluvio-marin, en contact permanent avec le plateau littoral qui sont les plus touchés; de plus, l'importance de la maladie est en relation directe avec la pluri-activité des populations. En revanche, les habitants du massif gréseux, agriculteurs ou éleveurs, aux systèmes de production moins complexes, restent très faiblement touchés.

\section{Le littoral guinéen: un milieu complexe aux interprétations économiques complémentaires}

L'espace littoral guinéen se caractérise par la juxtaposition et l'imbrication d'éléments géomorphologiques contrastés: espaces exondés/espaces inondables ou inondés; espaces marins ou fluviaux; zones salées/zones douces, en permanence ou temporairement. 
Tableau 5.

Résultats des prospections de 1997 et 2005

Préfecture de Dubreka

\begin{tabular}{|c|c|c|c|c|c|c|c|c|c|c|c|c|}
\hline \multirow[t]{2}{*}{ Village } & \multicolumn{6}{|c|}{1997} & \multicolumn{6}{|c|}{2005} \\
\hline & Recensés & Visités & Séro + & $\mathrm{T}+$ & $\begin{array}{l}\text { Séro } \\
\text { prévalence }\end{array}$ & Prévalence & Recensés & Visités & Séro + & $\mathrm{T}+$ & $\begin{array}{c}\text { Séro } \\
\text { prévalence }\end{array}$ & Prévalence \\
\hline Arabanty & 176 & 138 & 4 & 1 & 2,9 & 0,72 & & & & & & \\
\hline Bagueya & 376 & 166 & 0 & 0 & 0 & 0 & 495 & 105 & 0 & 0 & 0 & 0 \\
\hline Boneya & 293 & 222 & 3 & 1 & 1,35 & 0,45 & 398 & 113 & 0 & 0 & 0 & 0 \\
\hline Dantéforé & 198 & 224 & 2 & 0 & 0,89 & 0 & 258 & 178 & 0 & 0 & 0 & 0 \\
\hline Dembaya & 736 & 416 & 0 & 0 & 0 & 0 & & & & & & \\
\hline Dofili & 95 & 53 & 0 & 0 & 0 & 0 & & & & & & \\
\hline Doty & 203 & 119 & 0 & 0 & 0 & 0 & & & & & & \\
\hline Gbantama & 803 & 404 & 0 & 0 & 0 & 0 & & & & & & \\
\hline Gbensenké & 434 & 170 & 2 & 2 & 1,18 & 1,18 & 634 & & & & & \\
\hline Kangoléah & 962 & 463 & 3 & 2 & 0,65 & 0,43 & 716 & & & & & \\
\hline Kanka & 300 & 144 & 4 & 4 & 2,78 & 2,78 & & & & & & \\
\hline Kholaya & 694 & 324 & 1 & 1 & 0,31 & 0,31 & 604 & 319 & 2 & 1 & 0,63 & 0,31 \\
\hline Khorira & 1363 & 527 & 3 & 0 & 0,57 & 0 & & & & & & \\
\hline
\end{tabular}




\begin{tabular}{|c|c|c|c|c|c|c|c|c|c|c|c|c|}
\hline \multirow[t]{2}{*}{ Village } & \multicolumn{6}{|c|}{1997} & \multicolumn{6}{|c|}{2005} \\
\hline & Recensés & Visités & Séro + & $T_{+}$ & $\begin{array}{c}\text { Séro } \\
\text { prévalence }\end{array}$ & Prévalence & Recensés & Visités & Séro + & T+ & $\begin{array}{c}\text { Séro } \\
\text { prévalence }\end{array}$ & Prévalence \\
\hline Kopering & 205 & 190 & 1 & 1 & 0,53 & 0,53 & 267 & 226 & 2 & 2 & 0,88 & 0,88 \\
\hline Khonikourou & & 0 & 0 & 0 & & & 165 & 137 & 1 & 1 & 0,73 & 0,73 \\
\hline Limbita/Téré & 623 & 503 & 3 & 1 & 0,60 & 0,20 & 676 & 444 & 0 & 0 & 0,00 & 0,00 \\
\hline Magnokhoun & 950 & 714 & 4 & 1 & 0,56 & 0,14 & 989 & 529 & 11 & 9 & 2,08 & 1,70 \\
\hline Mengueya & 707 & 435 & 5 & 5 & 1,15 & 1,15 & 920 & 272 & 2 & 1 & 0,74 & 0,37 \\
\hline Missira & 600 & 207 & 3 & 0 & 1,45 & 0 & 319 & 226 & 0 & 0 & 0 & 0 \\
\hline Sanko & 168 & 147 & 1 & 1 & 0,68 & 0,68 & 297 & & & & & \\
\hline Siraya & 341 & 269 & 6 & 4 & 2,23 & 1,49 & 411 & 422 & 16 & 8 & 3,79 & 1,90 \\
\hline Taban & 563 & 437 & 6 & 3 & 1,37 & 0,69 & 474 & 317 & 1 & 1 & 0,32 & 0,32 \\
\hline Tabéta & 303 & 201 & 6 & 3 & 2,99 & 1,49 & & & & & & \\
\hline Tayiré & 445 & 280 & 1 & 1 & 0,36 & 0,36 & 301 & & & & & \\
\hline Tégna & 552 & 405 & 16 & 11 & 3,95 & 2,72 & 501 & 168 & 1 & 1 & 0,60 & 0,60 \\
\hline Tounguissour & 150 & 114 & 14 & 6 & 12,28 & 5,26 & & & & & & \\
\hline Yatagui & & 63 & 3 & 2 & 4,76 & 3,17 & & & & & & \\
\hline Yattia & 244 & 223 & 2 & 2 & 0,9 & 0,9 & 320 & 125 & 1 & 0 & 0,80 & 0,00 \\
\hline Yéguéa & & 281 & 6 & 4 & 2,14 & 1,42 & & & & & & \\
\hline
\end{tabular}

Sources: LAVEISSIERE et al., 1997; Rapport PNLTHA, 2005. 
On peut, grossièrement, y adopter la classification suivante: le massif gréseux ou granitique en arrière de la plaine littorale, souvent abusivement qualifiée d'arrière-mangrove, plaine côtière exondée; les zones inondables avec les plaines fluviales, les plaines d'estuaires supérieurs, imbriquées dans la plaine littorale, puis les plaines d'estuaires inférieurs jouxtant la mangrove de front de mer. La permanence et l'importance de la salinité des sols inondables dépendent de leur situation topographique et géographique. Enfin, les eaux marines et fluviales se rencontrent diversement selon les saisons dans la mangrove.

En outre, la combinaison entre plaines littorales et mangroves (à Avicenia ou à Rhizophora) n'est pas homogène d'une région à une autre et la part respective de chacune d'elle varie considérablement selon les régions; Dubreka par exemple se caractérise par une plaine littorale étroite - parfois moins de $2 \mathrm{~km}$ - faisant face à des mangroves où les espaces non accessibles aux grandes marées sont très rares. On y trouve aussi, peut-être en raison des difficultés d'y développer une poldérisation importante, le plus beau massif forestier de mangrove régional. Ailleurs, les plaines littorales sont beaucoup plus larges et les parties insubmersibles situées au coeur des mangroves plus importantes - Coyah, Boffa et Forécariah et leur mise en valeur depuis le début du siècle porte les marques de ces différences.

De plus, les divers éléments géomorphologiques présentent des potentiels pathologiques différents à travers leurs capacités variées à assurer la reproduction et la survie de la mouche tsé-tsé, vecteur de la maladie du sommeil.

\section{Le littoral guinéen: un espace interprété en termes de complémentarité par les populations}

La majorité de la population régionale réside sur la plaine littorale - 45 villages sur 51 à Dubreka - malgré l'exiguité de celle-ci et seulement $7 \%$ de la population se livre à temps plein à des activités de production dans les mangroves, utilisant pour ce faire des villages permanents ${ }^{5}$. Cependant, une part importante de la population 
"de terre ferme » - variable selon les zones et les années - utilise des campements temporaires ou les villages permanents, pour se livrer à la pluri-activité : pêche, collecte du sel, bûcheronnage, culture du riz sur des "bougounis " ${ }^{6}$ aménagés font partie des activités pratiquées en mangrove et concernent annuellement environ $1 / 5$ de la population de la préfecture de Dubreka. Or, on sait que la population utilisatrice de la mangrove n'est pas, en termes familiaux et individuels, toujours la même. De ce fait, les mêmes lieux peuvent, selon les saisons, accueillir des familles différentes n'y pratiquant pas les mêmes activités.

Ces activités complètent l'arboriculture, les cultures pluviales manioc, arachide, légumes, plantes à sauce... - et la riziculture de bas-fond, fluviale ou d'estuaire supérieur pratiquée sur le plateau littoral ou d'estuaire inférieur pratiquée à la limite des deux zones.

Ces occupations complémentaires, spatialement et temporellement dissociées, entraînent ponctuellement dans le temps et d'une manière diurne, un accroissement de la présence humaine le long de la ligne de partage entre la terre ferme d'une part et la mangrove et ses chenaux d'autre part; c'est un écotone où les vecteurs de la maladie du sommeil ne sont pas durablement soumis aux problèmes liés à la salinité des eaux ou des terres. C'est là qu'embarquent ou débarquent les utilisateurs de la mangrove résidant sur la terre ferme; c'est là que l'on débarque le bois, le poisson, le riz ou le sel; c'est là aussi que l'on implante les aires de battage du riz; c'est là, enfin, que, de préférence, sont situées les pépinières et les cultures maraîchères qui bénéficient des résurgences des eaux continentales emmagasinées dans les failles, fractures et diaclases du plateau gréseux ou du vieux socle granitique, situées en amont.

Les stratégies diversifiées (tant pour des raisons physiques simple disponibilité ou accroissement trop important de la salinité des bougounis par exemple -, que sociales ou circonstancielles choix de privilégier telle ou telle production en raison du rapport attendu ou de la disponibilité en main-d'œuvre) développées par les divers exploitants (RIBEREAU, 1996), les amènent à se déplacer 
d'une manière importante et variable et à séjourner diversement à l'intérieur des différents espaces physiques mais économiquement complémentaires de l'espace régional. Ce faisant, ils s'exposent plus ou moins aux risques d'infection par le parasite de la maladie du sommeil, au sein des multiples «espaces partagés ", espaces à risque, générés par les activités humaines et les mobilités induites (HeRVOUÉT et al., 2004).

Si les diverses potentialités épidémiogènes ${ }^{7}$, des espaces et des lieux, résultant des interprétations différenciées que font les hommes de ce milieu complexe qu'est le littoral guinéen, participent à expliquer la distribution hétérogène de la maladie du sommeil dans l'espace et le temps, elles ne justifient en rien le maintien de la maladie, malgré la réalisation de nombreuses prospections.

\section{Une situation inquiétante: malgré la lutte la maladie est toujours présente}

Dans la préfecture de Dubreka, 48 villages du littoral ont bénéficié d'au moins une prospection médicale concernant la maladie du sommeil depuis 1997. L'un d'entre eux - Siraya - a même été visité à six reprises entre 1997 et 2006 (tabl. 6), dont quatre fois au cours des cinq dernières années; la prévalence reste élevée, avec $3 \mathrm{NT}^{8}$ en 2006 sur 265 personnes vues dont un sans ganglion, preuve probable que le parasite circule toujours (malheureusement, les équipes de prospections ne disposent jamais d'un listing des habitants visités les années précédentes; la disposition d'un recensement informatisé et réactualisé à chaque prospection lèverait ce handicap).

Malgré ce travail considérable, il n'a pas été enregistré de baisse significative de la prévalence de la maladie dans les six villages ayant bénéficié d'au moins quatre prospections en 10 ans (tabl. 6). De plus, chaque année, des malades originaires de ces villages se

7 Potentialités épidémiogènes qui restent largement à étudier à partir des potentialités épidémiques des divers «milieux naturels» constitutifs du littoral guinéen et des diverses mobilités humaines.

8 Dont un NT dépisté à Magnokhoun bien que résidant à Siraya. 
Tableau 6.

Nombre de prospections par village de 1997 à 2006 dans la préfecture de Dubreka

\begin{tabular}{|crlr|}
\hline Nombre de prospections & Villages & Population estimée en 2006 \\
\hline 6 & 1 & Siraya & 411 hab. \\
5 & 2 & Boneah, Tégna & 1052 hab. \\
4 & 4 & Yatiya, Kholaya, Kanagban & 2591 hab. \\
3 & 4 & Bagueah, Magnokhoun, & \\
& & Missira, Taban, Mengueya & 3535 hab. \\
2 & 15 & & 6631 hab. \\
1 & 22 & & 1493 hab. \\
\hline
\end{tabular}

présentent spontanément à l'hypnoserie de Dubreka pour se faire soigner. Si nous prenions en compte ces malades confirmés, cela augmenterait considérablement les prévalences affichées lors des prospections seules. À titre d'exemple, pour le seul village de Siraya, 9 hommes et 3 femmes ont été dépistés par les prospections

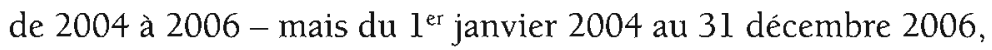
7 hommes et 2 femmes se sont présentés spontanément à l'hypnoserie de Makompo pour se faire traiter. Soit un total de 21 nouveaux trypanosomés, ce qui représente $5,11 \%$ de la population recensée. Et cela bien que les prospections n'aient jamais atteint dans ce village un taux de présentation supérieur à $70 \%$ !

Tableau 7.

Résultats des prospections dans les six villages les plus visités ${ }^{9}$

\begin{tabular}{|ccccc|}
\hline Année & Population visitée & NT & Prévalence & $\begin{array}{c}\text { Part pop. } \\
\text { résidante vue }\end{array}$ \\
\hline 1997 & 1609 & 19 & 1,18 & $67 \%$ \\
1998 & 2673 & 41 & 1,53 & $98 \%$ \\
2002 & 2882 & 42 & 1,45 & $80 \%$ \\
2003 & 1471 & 19 & 1,29 & $45 \%$ \\
2005 & 2624 & 16 & 0,75 & $54 \%$ \\
2006 & 205 & 2 & 0,69 & $50 \%$ \\
\hline
\end{tabular}

Source: SIMARRO, 2005.

9 Pour 2006, les individus vus dans tel ou tel village, dont les élèves des écoles, ont été rapportés à leur village d'habitat, selon les recommandations du Manuel de lutte contre la maladie du sommeil. 
Les calculs du tableau 7 ont été réalisés sur la base du RGPH de 1996 comparés aux recensements réalisés par l'équipe «trypanopanosomiase » de Guinée en 1996-1997, puis en 2007, et sur la base d'une croissance annuelle de $2,8 \%$.

Si l'on exclut les merveilleux scores de 1998 et de $2002^{10}$, il apparaît que ces prospections n'atteignent jamais $75 \%$ de la population résidante. Or il a été montré, en Côte d'Ivoire, que lorsqu'on visitait, globalement $75 \%$ d'une population, $50 \%$ des porteurs de parasites échappaient au dépistage (HERVOUËT, 1983). Et si dans ce faciès épidémiologique particulier qu'est le littoral, comme en zone forestière de Côte d'Tvoire, la moitié des malades se trouvent dans les $25 \%$ de la population qui échappent à la prospection, une partie de la réponse à la question de la cause du maintien du niveau d'endémicité est trouvée. Mais la situation est plus complexe.

Les résultats de 2006 sont difficilement comparables à ceux des autres années puisque cette année-là, sur les six villages les plus touchés par la maladie, seul le village de Siraya, l'un des mieux sensibilisés de toute la région, a été visité. Malgré cette situation particulière, seulement $70 \%$ des habitants se sont présentés au prélèvement.

\section{La simplification des protocoles}

C'est donc la première prospection, celle de 1997 qui a réalisé le meilleur score de présentation des populations à la prospection. Il faut aussi ajouter qu'elle se distingue des autres par le fait qu'elle fut la seule ${ }^{1 \mathbf{I}}$ à être précédée d'un recensement nominatif de

10 Pour 1998 et 2002 , comme pour les autres années, ces calculs ont été réalisés sur la population des " secteurs », synonymes, pour nous, des villages concernés. Mais il semble que, pour ces deux années, au moins, le nom donné se rapporte plus à des districts, regroupant plusieurs secteurs: Bagueah, Kanagban, etc., ou à des lieux de rassemblement drainant des localités extérieures, espaces n'ayant pas de valeur épidémiologique particulière. A titre d'exemple, à Kanagban, il fut visité 1337 habitants alors que le recensement de 1996 ne créditait ce secteur que de 1044 habitants; de même à Siraya 589 visités pour 330 résidants. .

11 Exceptée la prospection aux îles de Los en 2006. 
la population à visiter. Munies des listings nominatifs familiaux, les équipes (LAVEISSIÈRE et al., 1997) ont mené la prospection selon un protocole longuement rodé en Côte d'Ivoire depuis 1979 et bien décrit dans le Manuel de lutte contre la maladie du sommeil (LAVEISSIĖRE et PENCHENIER, 2002).

Malheureusement, ces cahiers n'ont pas été conservés pas plus d'ailleurs que les fiches d'identification des personnes examinées qui, jusqu'en 2005, furent détruites immédiatement après la promulgation des résultats sérologiques. Pour ces raisons, il n'est jamais possible, avant 2006, d'analyser la structure par sexe et par âge de la population réellement examinée.

En 1998, le protocole élaboré pour les prospections fut abandonné, ou du moins simplifié, et si le terme de "recensement " de la population est conservé dans les rapports, il ne s'agit plus, en fait - confusion dramatique en termes d'analyse épidémiologique que d' "enregistrements" des populations se présentant à la prospection $^{12}$. La démarche simplificatrice est d'ailleurs bien décrite par Camara: Le schéma de dépistage est celui classiquement utilisé en Afrique de l'Ouest: après une phase de sensibilisation (...) l'équipe de prospection vient s'installer dans le village choisi. Chaque personne présente est recensée et reçoit une convocation personnelle (CAmARA et al., 2005) en oubliant qu'un recensement passe par le déplacement des recenseurs au lieu d'habitat des populations concernées et par l'enregistrement nominatif de l'ensemble de la population résidante, y compris les individus temporairement absents $^{13}$. De ce fait, il devient très difficile de faire une bonne

12 De 1998 et jusqu'à 2005, il n'y eut plus d'encadrement de la jeune équipe "trypano» de Guinée par des chercheurs et médicaux chevronnés en santé publique. A partir de 2005, cet encadrement fut réalisé par de jeunes chercheurs néophytes en santé publique et surtout préoccupés de recherche fondamentale. La mainmise actuelle des vétérinaires sur les recherches fondamentales, non seulement sur les trypanosomoses animales mais aussi sur les trypanosomiases humaines, n'est qu'un facteur aggravant.

13 L'absence de réalisation de recensements est aujourd'hui justifiée par le coût de ceux-ci. C'est oublier qu' « une bonne connaissance de la population » (JAMOT, 1935) est indispensable au contrôle de la maladie et «fait partie de la prospection ». Rappelons, aussi, que le fait de disposer d'un recensement nominatif réduit de 30 à $50 \%$ le temps de présence sur le terrain des 10 ou 12 personnes constitutives de l'équipe de prospection. Ce qui revient à dire que réaliser un recensement préalable à la prospection réduit le coût de celle-ci... 
utilisation scientifique et épidémiologique des données collectées car la population qui se présente aux prospections n'est pas représentative, démographiquement et épidémiologiquement, de la population résidante. Il s'agit là d'un véritable masque qui ne permet pas au service de lutte contre la maladie d'auto-évaluer correctement son action. Ainsi en 1998, la population dite « recensée" était de 7554 habitants et la population vue de 7376 individus, soit un taux de présentation de $98 \%$ ! (CAMARA, 1998). Le PNLTHA ayant pris conscience de ce problème en 2005, toute la documentation établie depuis 1997 a été recherchée, réunie, sauvegardée puis analysée pour approcher les structures des populations visitées et tenter de comprendre les raisons profondes du maintien de l'endémie malgré les efforts entrepris.

Nous disposons ainsi de 5 types de documents: des recensements de la population, des cahiers d'enregistrement - et non de recensement - des populations s'étant présentées, des fiches d'identification des personnes ayant été prélevées, des livres « labo » journaliers et enfin, des rapports de prospection ou de publications (cf. tabl. 8)

Nous reviendrons ultérieurement sur l'utilité des recensements nominatifs. Quant aux cahiers d'enregistrement - qualifiés de recensements -, ils ne peuvent être mis en relation avec les populations réellement examinées, jusqu'en 2006, que pour les chiffres globaux: nombre d'habitants enregistrés/nombre d'individus réellement vus. La différence entre les nombres absolus traduit, essentiellement, le nombre de chefs de famille que les "recenseurs»

Tableau 8.

Documents disponibles pour analyser les résultats des prospections

\begin{tabular}{|cccccc|}
\hline & Recensement & $\begin{array}{c}\text { Cahiers } \\
\text { enregistrement }\end{array}$ & $\begin{array}{c}\text { Fiches } \\
\text { identification }\end{array}$ & $\begin{array}{c}\text { Livre } \\
\text { «labo " }\end{array}$ & $\begin{array}{c}\text { Rapport ou } \\
\text { publication }\end{array}$ \\
\hline 1997 & oui & non & non & non & oui \\
1998 & non & oui & non & non & oui \\
2002 & non & non & non & oui & non \\
2003 & non & oui & non & oui & oui \\
2004 & non & oui & non & oui & non \\
2005 & non & oui & non & oui & oui \\
2006 & oui & oui & oui & oui & oui \\
\hline
\end{tabular}


ont simplement enregistré pour identifier les familles, mais qui était absents. Les «livres Labo" des prospections permettent de retrouver l'âge, le sexe, le village d'origine des suspects sérologiques et des nouveaux trypanosomés dépistés. Les rapports et les publications présentent des données très hétérogènes, mais permettent de combler les lacunes résultant des absences des autres documents.

Globalement, il est impossible - sauf en 2006 - de reconstituer la structure de la population réellement vue par l'équipe de prospection, car, nous l'avons dit, les fiches d'identification ont été jetées. Cependant, lorsque les cahiers d' «enregistrement» de la population ont été conservés, ils permettent d'approcher cette réalité comme le montre le tableau 9 qui indique, pour chaque prospection le sex-ratio de la population "enregistrée", puis celui des habitants de plus 50 ans, de ceux de 15 à 49 ans et enfin, celui des enfants de moins de 15 ans.

Tableau 9 .

Sex-ratio (nombre d'hommes pour 100 femmes)

des populations recensées, enregistrées et prélevées selon l'année

\begin{tabular}{|c|c|c|c|c|c|c|}
\hline & $\begin{array}{l}\text { Population } \\
\text { recensée }\end{array}$ & Sex-ratio & $\begin{array}{c}\text { SR } \\
+50 \text { ans }\end{array}$ & $\begin{array}{c}\mathrm{SR} \\
\text { adultes }\end{array}$ & $\begin{array}{c}S R \\
-15 \text { ans }\end{array}$ & $\begin{array}{c}\% \mathrm{CF} \\
\text { absents }\end{array}$ \\
\hline 1997 & 9678 & 94 & 95 & 85 & 104 & \\
\hline 2006 îles Los & 6192 & 103 & 106 & 98 & 109 & \\
\hline \multirow[t]{2}{*}{2007 épicentre } & 4719 & 93 & 88 & 74 & 115 & \\
\hline & $\begin{array}{l}\text { Population } \\
\text { enregistrée }\end{array}$ & Sex-ratio & $\begin{array}{c}\text { SR } \\
+50 \text { ans }\end{array}$ & $\begin{array}{c}S R \\
\text { adultes }\end{array}$ & $\begin{array}{c}\text { SR } \\
-15 \text { ans }\end{array}$ & $\begin{array}{c}\% \mathrm{CF} \\
\text { absents }\end{array}$ \\
\hline 1998 & 7540 & 82 & 95 & 61 & 104 & $58 \%$ \\
\hline 2003 Dubreka & 3755 & 83 & 110 & 49 & 110 & $30 \%$ \\
\hline 2003 Boffa & 7059 & 79 & 107 & 57 & 93 & $29 \%$ \\
\hline 2005 Dubreka & 4835 & 81 & 110 & 53 & 99 & $54 \%$ \\
\hline 2006 îles Los & 4783 & 90 & 85 & 73 & 105 & $10 \%$ \\
\hline \multirow[t]{2}{*}{2006 épicentre } & 3742 & 90 & 81 & 50 & 120 & $35 \%$ \\
\hline & $\begin{array}{l}\text { Population } \\
\text { ponctionnée }\end{array}$ & Sex-ratio & $\begin{array}{c}\text { SR } \\
+50 \text { ans }\end{array}$ & $\begin{array}{c}\text { SR } \\
\text { adultes }\end{array}$ & $\begin{array}{c}\text { SR } \\
-15 \text { ans }\end{array}$ & $\begin{array}{c}\% \text { CF } \\
\text { absents }\end{array}$ \\
\hline 2006 îles Los & 4361 & 87 & 89 & 65 & 110 & \\
\hline 2006 épicentre & 3630 & 88 & 70 & 47 & 112 & \\
\hline Sex-ratio « Guir & inée rurale » & 94 & 101 & 80 & 105 & \\
\hline
\end{tabular}

Source: Enquête démographique et de santé, 1999 
Il faut noter que les pourcentages de chefs de familles absents à la prospection sont calculés à partir des familles venues se présenter à la prospection, et non pas par rapport aux familles réellement résidantes et que nous ne connaissons pas! Ces pourcentages d'absents ont varié, selon les villages, de 8 à $65 \%$ en 1998, de 9 à $77 \%$ en 2003, de 7 à $79 \%$ en 2004 et de 7 à $48 \%$ en 2005 .

Seul le recensement de 1997, puis les «enregistrés» des îles de Los présentent une pyramide des âges proche de celle établie pour la Guinée rurale; pour Dubreka, nous ne savons rien de celle de la population réellement vue.

Il manque toujours beaucoup plus d'hommes que de femmes à la prospection et ceci est surtout vrai pour les 15-50 ans avec un pic dans certaines tranches d'âge comme les $35-45$ ans où $60 \%$ des hommes peuvent être absents. Ce qui n'est pas sans conséquences épidémiologiques.

Ces chiffres, basés sur l'enregistrement des populations et non pas les populations réellement vues, surévaluent encore la présentation des habitants et notamment des hommes, comme le montre le tableau 10, établi à partir des prospections de 2006.

Le sex-ratio (nombre d'hommes pour cent femmes) diminue des recensés vers les populations réellement vues en passant par les enregistrés montrant par là, qu'à toutes les étapes du processus de dépistage, les hommes "s'échappent» pour ne pas subir de prélèvement. De ce fait, chez les adultes, ce sont en général moins de $50 \%$, sinon $30 \%$, des hommes qui sont vus par la prospection, alors que ces groupes d'âge sont les plus touchés par la maladie, notamment les chefs de famille qui se contentent souvent de faire acte de présence dérisoire.

Ainsi, à propos de la prospection de 2002 - dont les données de base n'ont pas été retrouvées -, il est juste de dire, en valeur absolue, que «sur les 96 trypanosomés, il y a autant de femmes que d'hommes atteints" (CAMARA et al., 2005: 158), mais ce ne l'est pas en valeur relative, car les hommes sont significativement plus touchés que les femmes. Dans ce système complexe où les lieux ne sont pas neutres, pas plus que les espaces ne sont indifférents aux phénomènes pathologiques qui les affectent (HERVOUËT, 1990), les tableaux précédents, tout comme le suivant 
Tableau 10 .

Distribution de la population et sex-ratio selon l'étape de la prospection

\begin{tabular}{|c|c|c|c|c|c|c|c|c|c|}
\hline \multirow{2}{*}{$\begin{array}{l}\text { Population } \\
\text { (nombre de } \\
\text { personnes) }\end{array}$} & \multicolumn{3}{|c|}{ recensée } & \multicolumn{3}{|c|}{ enregistrée } & \multicolumn{3}{|c|}{ prélevée } \\
\hline & +50 & adultes & -15 ans & +50 & adultes & -15 ans & +50 & adultes & -15 ans \\
\hline Hommes & 336 & 1536 & 1254 & 237 & $892(58 \%)$ & 1328 & 226 & $718(47 \%)$ & 1075 \\
\hline \multicolumn{10}{|l|}{ îles de Los } \\
\hline Femmes & 315 & 1563 & 1150 & 279 & $1216(78 \%)$ & 1265 & 254 & $1106(71 \%)$ & 973 \\
\hline Sex-ratio & 106 & 98 & 109 & 85 & 73 & 105 & 89 & 65 & 110 \\
\hline Hommes & 261 & 828 & 1188 & 152 & 363 & 1262 & 128 & 332 & 1237 \\
\hline \multicolumn{10}{|l|}{ Dubreka } \\
\hline Femmes & 297 & 1116 & 1029 & 188 & 729 & 1048 & 184 & 706 & 1040 \\
\hline Sex-ratio & 88 & 74 & 115 & 81 & 50 & 120 & 70 & 47 & 112 \\
\hline
\end{tabular}

Sources: îles de Los: Recensement et prospection en 2006; Dubreka: Prospection 2006, recensement 2007. 
ainsi que la figure 3, montrent, de la même manière, qu'âges et sexes, au travers des activités journalières des individus et des mobilités qu'elles impliquent fixées par le statut social, sont des déterminants importants des degrés d'endémicité des populations. En outre, tous sexes confondus, ce sont les classes d'âge les plus touchées par la maladie qui se présentent le plus mal aux prospections. Qu'elle est alors la prévalence réelle chez les hommes de 30 à 50 ans? Et au sein de ces classes d'âge, combien laisse-t-on de trypanosomés, réservoirs du parasite pouvant infecter de nouvelles glossines, dans la nature? En 2005, sur 8 chefs de familles trouvés trypanosomés à Magnokhoun, 7 avaient échappé à toutes les prospections réalisées dans l'épicentre du foyer depuis $1997 \ldots$

En ne voyant qu'une faible part des populations les plus à risque, le PNLTHA ne peut que limiter la gravité du péril trypanique sur le littoral guinéen. 11 est alors limité à une action curative et non pas préventive.

Tableau 11.

Prévalences par groupes d'âge selon les années de prospection

\begin{tabular}{|c|c|c|c|c|c|}
\hline \multicolumn{2}{|c|}{ Population enregistrée } & \multirow[t]{2}{*}{ NT } & \multicolumn{3}{|c|}{ Prévalences } \\
\hline & & & +50 ans & adultes & -15 ans \\
\hline $\begin{array}{c}\mathrm{H} \\
\text { 1998, Dubreka }\end{array}$ & 3452 & 32 & 0,29 & 2,24 & 0,42 \\
\hline & 4088 & 19 & 0,55 & 0,72 & 0,24 \\
\hline 2003, Dubreka & 1466 & 17 & 0,65 & 2,94 & 0,53 \\
\hline$F$ & 1840 & 16 & 3,67 & 0,94 & 0,46 \\
\hline 2003, Boffa & & & 1,11 & 1,29 & 0,34 \\
\hline$F$ & 3174 & 24 & 0,58 & 1,28 & 0,39 \\
\hline 2005, Dubreka & & 24 & 0,73 & 3,04 & 0,31 \\
\hline$F$ & 2668 & 13 & 0,40 & 0,99 & 0,08 \\
\hline 2005 , Boffa & & 14 & 0,79 & 0,80 & 0,28 \\
\hline$F$ & 3500 & 9 & 0,24 & 0,26 & 0,26 \\
\hline 2006, Dubreka & 1698 & 8 & 0,78 & 1,51 & 0,16 \\
\hline$F$ & 1932 & 6 & 0,54 & 0,42 & 0,19 \\
\hline
\end{tabular}


Figure 3.

Recensement et prospection en zone littorale guinéenne:

l'épicentre du foyer de Dubreka, 2006.
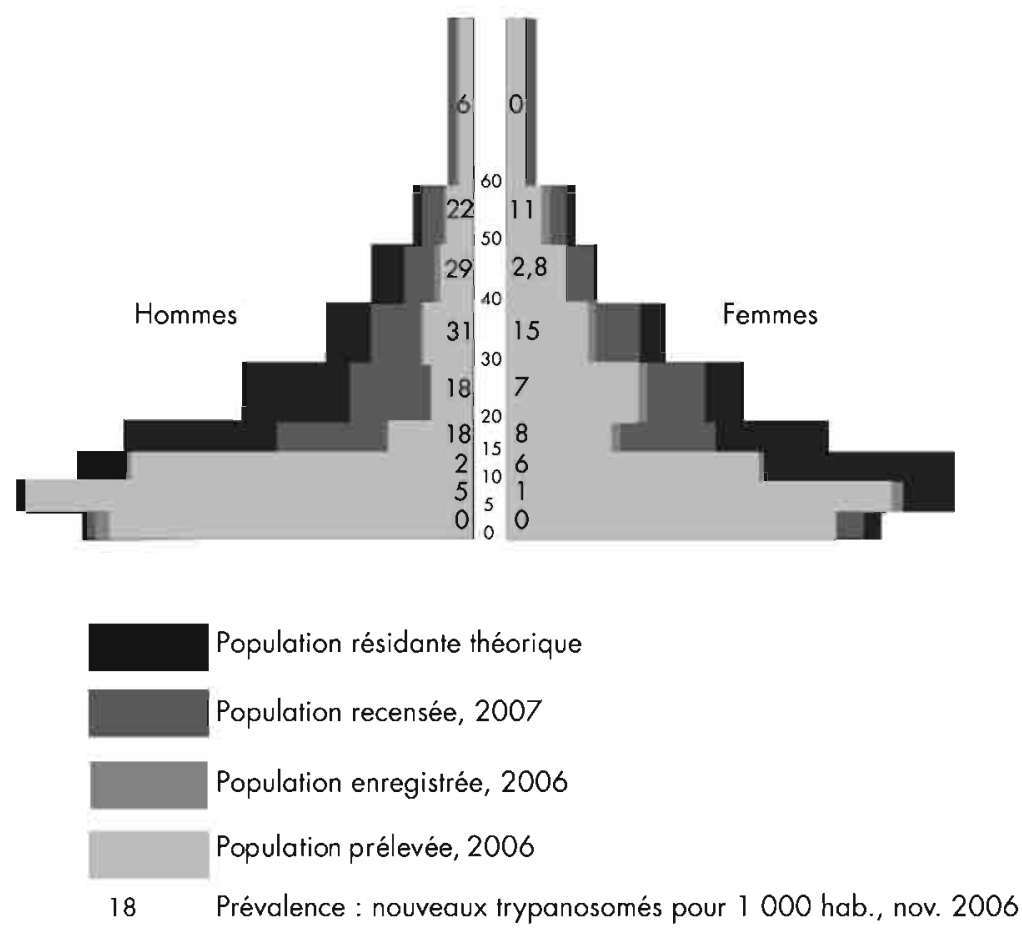

Le contrôle de la situation passe donc impérativement par celui des populations résidantes dont il faut améliorer considérablement la présentation aux prospections, tout en ciblant mieux les zones d'intervention prioritaires. Ce qui implique, en l'absence de discipline collective forte des populations et de présentation massive (plus de $85 \%$ ) aux prospections, de disposer d'un recensement nominatif des populations.

\section{Un recensement préalable de la situation améliore la présentation de la population aux prospections}

Disposer d'un recensement nominatif des populations est d'abord une nécessité si l'on veut identifier les groupes à risque et leur comportement vis-à-vis des équipes de contrôle de la maladie, 
c'est-à-dire l'impact réel, en termes épidémiologiques, du travail réalisé. Ensuite, l'établissement d'un tel document peut avoir des effets bénéfiques sur les résultats des prospections.

Figure 4.

Recensement et prospections en zone littorale guinéenne, îles de Los, 2006.
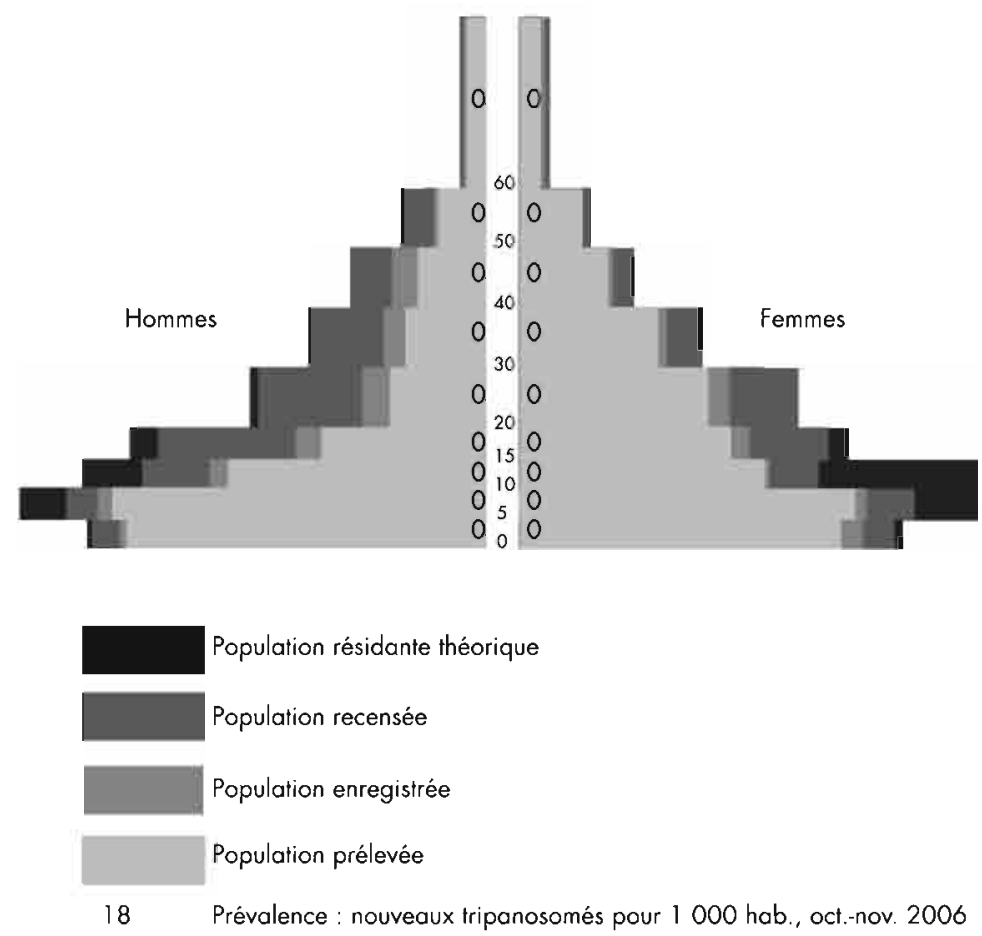

La comparaison des figures 3 et 4 est instructive, elle indique que la présentation des hommes à une prospection est bien meilleure lorsque celle-ci est précédée d'un recensement nominatif de la population ${ }^{14}$. Cela ne découle pas simplement du fait que passer de concession en concession, en expliquant le pourquoi de ce travail et le rôle épidémiologique des "porteurs sains" du parasite, constitue une excellente "sensibilisation», mais les chefs de famille se sachant identifiés et connus, ont moins tendance à s'échapper et alors encadrent mieux leurs dépendants.

14 Même si, sur les îles de Los, la modification au dernier moment du calendrier de travail pour une partie des villages s'est traduite par une diminution importante de la présentation. 


\section{Conclusion}

Durant des années, faute d'encadrement approprié, le travail de contrôle de la THA, réalisé en Guinée contre la maladie du sommeil n'a été ni analysé ni évalué. La simplification des protocoles de dépistage actif, la confusion entre des concepts comme «recensement » et «enregistrement " des populations, assimilable à une perte des savoir-faire, s'est traduite, sur le terrain, par le maintien de prévalences élevées, même là où les équipes sont passées quatre fois ou plus depuis dix ans.

Cependant, ces premiers travaux d'analyse de l'action du programme de lutte contre la maladie du sommeil indiquent qu'en se dotant des moyens nécessaires à l'évaluation de leurs activités, les programmes nationaux de lutte contre la maladie peuvent se placer en position d'organisme de santé publique, préventif, prévenant et non pas subissant la situation. Cette évolution éviterait, aussi, de ne choisir les lieux d'actions qu'en fonction des résultats des dépistages passifs, c'est-à-dire en ne demeurant que curatifs.

Deux niveaux d'actions interdépendantes se distinguent alors. En premier lieu celui de la détermination, caractérisation et hiérarchisation des espaces à risque et celui, stratégique, de la réalisation des dépistages qui, pour être efficient en absence de forte discipline collective des populations, nécessite que l'équipe de dépistage dispose de recensements nominatifs exhaustifs des populations, indiquant le sexe et l'âge ${ }^{15}$. Lorsque ceux-ci n'existent pas, il est nécessaire de les réaliser, en tenant compte de la toponymie relative aux habitats humains et amélioré, si possible, par le géo-référencement de toutes les concessions lorsque l'habitat est dispersé ou en nébuleuse. Le recensement permet de:

- connaître la population, quantitativement et qualitativement, population qui elle aussi se sait connue des prospecteurs;

- bien évaluer les besoins en matériel, en personne et en temps;

- couvrir la population environ deux fois plus rapidement que lorsque des fastidieux et bruyants «enregistrements» sont à réaliser ${ }^{16}$ et en échelonnant les convocations, limiter grandement les

$\mathbf{1 5}$ Les administrations coloniales en disposaient...

16 Et donc de réaliser des économies substantielles. 
attentes et par là les «fuites » d'individus par lassitude ou crainte des prélèvements;

- optimiser les luttes et les opérations de dépistage en identifiant les habitats, les personnes isolées, ainsi que celles ne s'étant pas présentées et qui, identifiées, pourront être convoquées.

Cela, à l'évidence, permet de déterminer la ou les stratégies à adopter afin d'atteindre les objectifs de santé publique fixés mais aussi d'assurer la maitrise des processus de diffusion, voire de propagation de la maladie.

Le recensement, une fois fait, n'est plus à refaire car à chaque prospection, il sera réactualisé, permettant l'évaluation permanente du travail réalisé en termes de couverture de la population à risque et de suivi des anciens trypanosomés.

En outre, ce travail ne bénéficie pas uniquement à la lutte contre la trypanosomiase humaine, mais à tous les projets visant au développement, à l'aménagement du territoire et à l'amélioration de la santé. À titre d'exemple, l'utilisation d'un recensement nominatif de la population, pour l'analyse des campagnes de vaccination, permettrait de ne plus voir des taux de présentation supérieurs à $100 \%$.

\section{Références bibliographiques}

CAMARA M., 1998 - Rapport d'activité. Ministère de la Santé publique, division Prévention. Doc. ronéo, 32 p.

Camara M., Kaba D., Kagbadouno M., Sanon J.-R., Ouendeno F., SOLANO P., 2005 - La trypanosomose humaine africaine en zone de mangrove en Guinée: caractéristiques épidémiologiques et cliniques de deux foyers voisins. Méd. Trop., 2005, 65 (2) : 156.

HERVOUËT J.-P., 1983 - Systèmes d'occupation du sol, conditions sociales et transmission de la trypanosomiase humaine. Orstom Petit Bassam, document multigraphié, 104 p., cartes.

HERVOUETT J.-P., 1990 - Le mythe des vallées dépeuplées par l'onchocercose. Géos, 18, 35 p.

Hervouét J.-P., LAfFly D., CARdon L., 2000 - La maladie du sommeil en Côte d'Ivoire: à la recherche d'indicateurs de risque. Espace, populations, sociétés, 2, "Maladies émergentes et reviviscentes " : 209-226. 
Hervouét J.-P., LAVEISSIĖRE C., 1987 - Les grandes endémies: l'espace social coupable. Politique Africaine, 28: 21-32.

HervouĖt J.-P., HANDSChumACHER P., LAFFLy D., 2004 - «Mobilités et espaces partagés au centre des risques sanitaires. L'exemple des grandes endémies tropicales. " In David G., éd. : Espaces tropicaux et risques. Du local au global, actes des $X^{\mathrm{e}}$ journées de géographie tropicale, Orléans, 24-26 sept., coll. du Cedete, PU Orléans/IRD, 127-136, ISBN 2-91345423-2.

HutChinson M. P., Barry El Hadj A., BREnGues J. R.J., 1964 - Rapport de la mission C.C.T.A. sur la trypanosomiase dans les territoires hissis et dans les régions frontières limitrophes, en Guinée, Sierra Leone et Liberia. Avril-juin 1964. Traduit de l'anglais par S. Grébaut et J. Brengues, document multigraphié, $138 \mathrm{p}$.

Jamot E., 1933 - Contribution à l'étude de la maladie du sommeil en Afrique occidentale française. Document multigraphié, $23 \mathrm{p}$.

JAMOT E., 1935 - Rapport du Médecin Colonel Jamot sur la maladie du sommeil en A.O.F. Document multigraphié, Gouvernement général de l'Afrique Occidentale Française, 27 p.

Laveissière C., Hervouét J.-P., Ott D., Camara M., Ouendeno F., 1997 Épidémiologie de la Trypanosomiase Humaine Africaine en zone de mangrove, Dubreka, République de Guinée. IPR/OCCGE, min. Santé publ. rép. Guinée, Doc. ronéo, $15 \mathrm{p}$.

LAVEISSIĖRe C., PenCHENIER L., 2002 - Manuel de lutte contre la maladie du sommeil. Volume 3: Dépistage. IRD/Oceac, IRD, $76 \mathrm{p}$.

OMS, 1962 - Comité d'experts de la trypanosomiase. Premier rapport. OMS, série des rapports techniques $n^{\circ} 247,60$.

OMS, 1979 - Les trypanosomiases africaines. OMS, série des rapports techniques $\mathrm{n}^{\circ} 635,118 \mathrm{p}$.

OMS, 1986 - La trypanosomiase africaine: épidémiologie et lutte. OMS, série des rapports techniques $\mathrm{n}^{\circ} 739,144 \mathrm{p}$.

PENCHENIER L. et al. 1996 - Historique et évolution de la maladie du sommeil dans les pays de l'Oceac. Bull. liais. Doc. Oceac, 29 (3) : 11-21.

Ribereau C., 1996 - Gestion des espaces littoraux et maladie du sommeil. L'exemple de Dubreka. Mémoire de maîtrise, université de Bordeaux-3, $145 \mathrm{p}$.

Simarro P., 2005 - Foyer de trypanosomiase humaine de Dubreka, étude de situation. Conakry, juin, doc. Powerpoint non publié. 



\section{Comportements humains et risque de transmission de la maladie du sommeil en zone périurbaine de Kinshasa (RDC)}

\section{Pascal GRÉBAUT, Gustave SIMO}

Émile Zola MANZAMBI, Jean-Marie BENA

Philémon MANSINSA, Gaëlle OLLIVIER, Gérard CUNY

La maladie du sommeil ou trypanosomose humaine africaine (THA) est une pathologie qui est mortelle si elle n'est pas soignée. Elle touche plusieurs dizaines de milliers de cas par an en Afrique subsaharienne, et l'on estime à 60 millions le nombre de personnes qui sont exposées (OMS, 2006). La pathologie se décline en deux stades. Le premier est un stade invasif qui fait suite à la piqûre infectante d'une glossine porteuse du pathogène (Trypanosoma brucei gambiense en Afrique centrale et de l'Ouest; $T$. $b$. rhodesiense en Afrique de l'Est), et durant lequel le malade ne présente pas de symptômes particuliers, mais sert de réservoir à trypanosomes et favorise la transmission aux proches lorsqu'il est piqué par une glossine susceptible d'héberger le cycle biologique du parasite. Le stade deux, se caractérise par le passage du parasite dans le système nerveux central après plusieurs mois, voire plusieurs années d'incubation dans le système sanguin; à ce stade commencent à apparaître de nombreux troubles psychomoteurs conduisant à un état d'asthénie permanente, qui est d'ailleurs à l'origine de l'appellation «maladie du sommeil», puis au décès du malade. 
Au deuxième stade, le traitement est douloureux et dangereux, et nécessite une hospitalisation. De plus, le dépistage demande les compétences d'une équipe spécialisée et équipée, ayant les moyens d'intervenir dans les zones rurales les plus reculées. Il est donc apparu que la rupture de la chaine de transmission par la lutte antivectorielle présentait l'avantage de limiter de façon efficace et à moindre coût le développement de l'endémie.

En matière de lutte contre la THA, la pratique de l'interdisciplinarité n'est vraiment apparue que dans les années 1980, avec l'expérience ivoirienne. À cette époque, la persistance de certains foyers endémiques en Côte-d'Ivoire, en dépit d'un suivi médical régulier, a poussé les chercheurs à dépasser le stade de l'approche médicale, limitée aux centres de dépistage et de traitement, pour aller s'intéresser aux pratiques sociales liées à la gestion du terroir au cœur de la zone endémique (HERVOUËT et LAVEISSIĖRE, 1987). L'un des préalables à cette recherche consistait à sortir des sentiers battus et aller chercher l'exposition au risque dans les plantations, au sein des populations allogènes échappant à la vigilance du système de santé classique. Il est apparu indispensable pour comprendre les mécanismes qui animent le complexe pathogène de la THA - constitué d'un hôte (humain ou animal), d'un vecteur (la glossine ou mouche tsé-tsé) et d'un parasite (le trypanosome) - de ne pas s'enfermer dans un domaine disciplinaire afin d'être en mesure d'appréhender les implications des différents acteurs et leurs interactions à tous les niveaux de la chaîne écologique.

La THA a longtemps été considérée comme une maladie spécifique aux zones rurales. En effet, en plus de la ré-émergence de l'endémie dans les anciens foyers historiques d'Afrique centrale et de l'Ouest (PEnCHenier et al., 1999), on observe depuis la fin du XX' l'apparition de nouveaux foyers endémiques. La désorganisation des systèmes de santé et l'héritage d'une situation endémique, apparemment maîtrisée après les indépendances, ont fortement contribué à diminuer la vigilance et ont favorisé l'apparition de nouvelles flambées endémiques (CATTAND, 2001). Plus récemment, d'autres types d'événements ont bouleversé les schémas classiques du contexte épidémiologique de la THA: les migrations pour raisons économiques ou pour fuir des conflits armés, les modifi- 
cations des paysages liées au développement des cultures de rente (café, cacao) (LoinTIER et al., 2001). Par ailleurs, un exode rural important a mis une pression démographique sans précédent sur les paysages urbains, exposant les citadins de quelques villes au risque de transmission (FOURNET et al., 2001; BILENGUe et al., 2001; COURTin et al., 2005).

Dans l'étude présentée ici, il s'agissait de répondre à une sollicitation du Programme national de lutte contre la THA (PNLTHA) en RDC qui voulait savoir si les cas de THA dépistés dans la ville de Kinshasa s'étaient contaminés ou non en périphérie de la ville, vérifiant ainsi l'hypothèse de l'existence d'un foyer local de THA dans une des mégapoles les plus importantes d'Afrique. L'ancien foyer de Kinshasa était considéré comme un foyer rural "éteint " depuis les années 1960 , et la surveillance de la maladie s'était relâchée. Il a fallu attendre la reprise des activités de dépistage en 1995 pour voir le nombre de cas régulièrement augmenter, jusqu'en 1999 où 912 cas ont été diagnostiqués (BILENGUÉ et al., 2001). Jusqu'en 1999, on considérait les patients atteints de Kinshasa comme des habitants ayant séjourné dans les provinces du Bas-Congo et du Bandundu, où la situation dans certains villages atteignait un niveau épidémique (VAN NIEWENHOVE et al., 2001). Mais c'est le nombre important de cas au premier stade de la maladie qui a attiré l'attention des autorités médicales, laissant suggérer que la contamination avait pu avoir lieu récemment et à proximité de la capitale. Plusieurs études furent menées qui décrivirent les activités de surveillance et de lutte (BILENGUÉ et al., 2001; EBEJA et al., 2003) ou qui tentèrent de mettre en évidence le caractère local de la transmission (RoBAYS et al., 2004; DE DEKEN et al., 2005) sans vraiment y parvenir. Notre étude s'était donc fixée comme objectifs d'évaluer en un an (2005) la présence du vecteur autour de la ville, l'importance du contact entre l'homme, la glossine et le pathogène, et enfin de déterminer les paysages à plus haut risque pour la transmission autour ou dans la ville. Elle s'est donc appuyée d'une part, sur l'entomologie et la biologie moléculaire pour la détermination des facteurs biotiques, et d'autre part, sur la géographie pour mieux comprendre l'interrelation entre ces facteurs biotiques et leur environnement. 


\section{Contexte de l'étude}

Kinshasa est la capitale de la République démocratique du Congo. C'est aussi une ville province. Pour notre étude, nous ne nous sommes intéressés qu'à la partie urbaine et périurbaine. Bordée au nord par le fleuve Congo, la ville repose sur une plaine alluviale et sablonneuse, et s'étend au sud, à l'est et à l'ouest sur des sites collinaires. Elle est parcourue par de nombreuses fractures, occupées par un réseau hydrique important, dont l'élément principal est la rivière Ndjili. La végétation naturelle, ou ce qu'il en reste, est constituée de forêt dégradée, de savane et de steppe. Les sols sont argileux à sablo-argileux et sableux. Les précipitations sont en moyenne légèrement supérieures à $1200 \mathrm{~mm}$ par an et la température moyenne annuelle est de $25^{\circ} \mathrm{C}$, le site bénéficie des masses d'air frais en provenance des courants du Benguela. La ville s'étend sur une superficie d'environ $450 \mathrm{~km}^{2}$ et

Figure 1.

Carte de l'agglomération de Kinshasa

et localisation des sites d'étude entomologique.

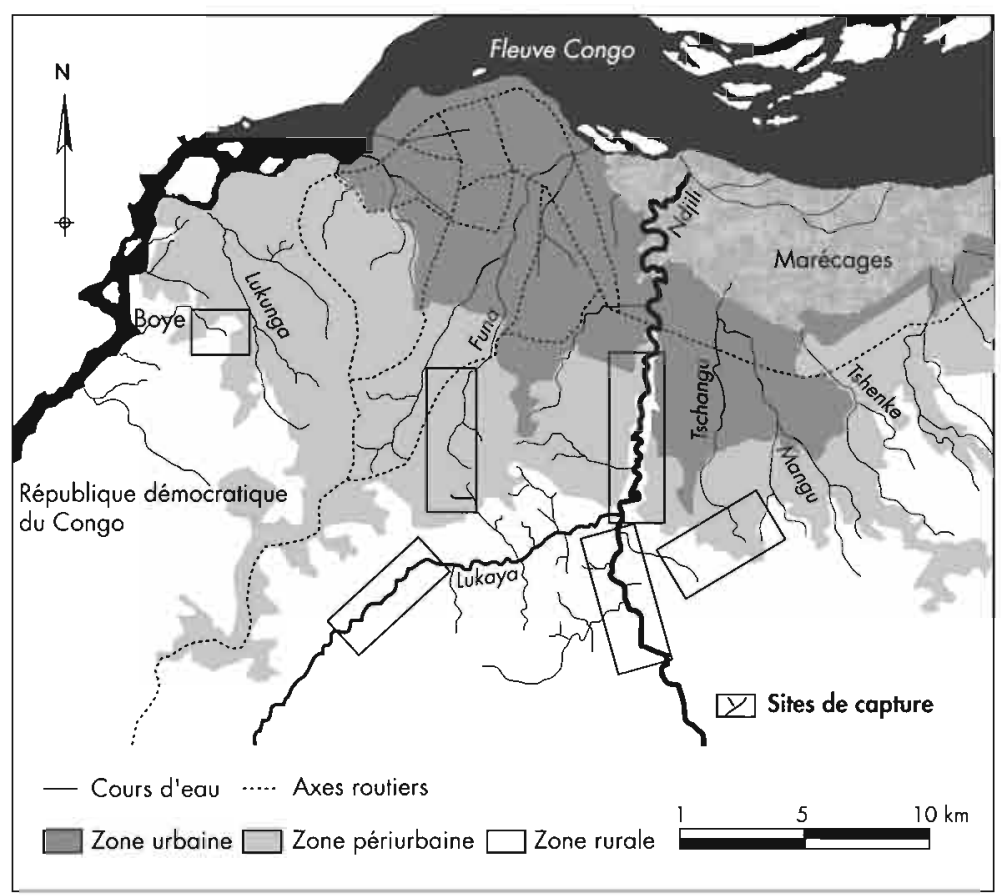


regroupe 24 communes. La population est estimée entre 4,5 et 5,5 millions d'habitants, avec une densité moyenne de 500 habitants au $\mathrm{km}^{2}$. La situation de crise économique qui perdure a poussé bon nombre de Kinois à exploiter les terres situées en périphérie de la ville.

Nous avons considéré que la glossine constituait le point focal de cette étude puisqu'en tant que vecteur, elle seule était capable de mettre en contact homme et pathogène. Deux enquêtes entomologiques ont donc été réalisées: la première en saison des pluies (février et mars 2005), dans un contexte climatique favorable aux glossines, la seconde, en saison sèche (juin et juillet 2005), plutôt défavorable aux populations de glossines. Huit sites d'étude ont été prospectés en saison des pluies, puis seulement six en saison sèche, deux sites s'étant avérés exempts de glossines en saison des pluies. Les insectes ont été capturés à l'aide de pièges pyramidaux (GOUTEUX et LANCIEN, 1986).

Photo 1.

Relevé de captures de glossines dans le site de Kimbanséké, en périphérie sud de Kinshasa.

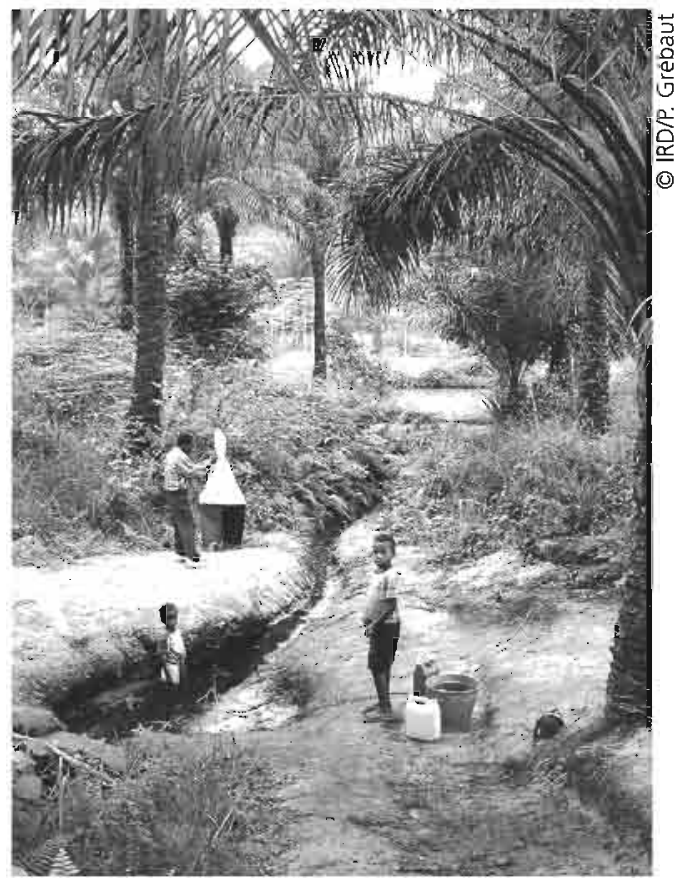


Photo 2.

Les habitants empruntent les voies d'eau pour aller poser les pièges.

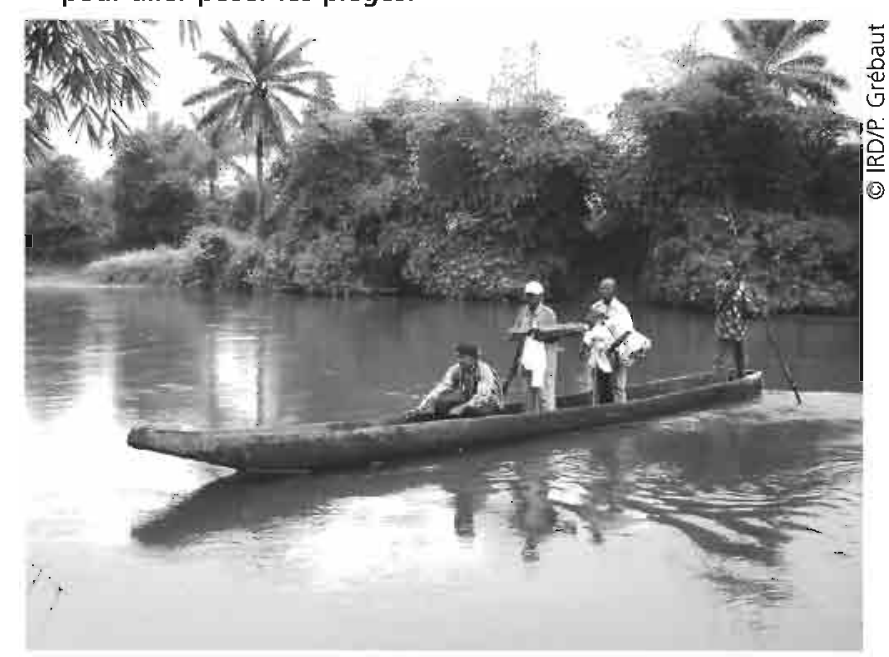

Sur chaque site, les captures étaient réalisées pendant quatre jours consécutifs avec une cinquantaine de pièges disposés tous les $300 \mathrm{~m}$ environ, afin d'éviter les interactions entre pièges. Les insectes étaient collectés deux fois par jour, pour disposer de mouches en bon état pour la dissection. Le traitement des glossines capturées est individuel et exhaustif, la première étape consistant à faire de visu un tri entre les mouches vivantes et mortes, les espèces et les sexes, et à identifier les glossines fraîchement écloses qui n'ont jamais pris de repas de sang (ténérales). Ces dernières constituent un groupe intéressant au titre d'indicateur des lieux de reproduction, mais aussi en tant que groupe particulièrement sensible à l'infection par les trypanosomes. Dans la seconde étape les insectes vivants sont disséqués afin d'isoler les organes pour la recherche de trypanosomoses et l'identification des hôtes nourriciers par PCR (amplification en chaîne par polymérisation) (MOSER et al., 1989; HERDER et al., 2002; NJIOKOU et al., 2004).

Pour chacun des 305 points de capture, les coordonnées géographiques (décimales) ont été relevées à l'aide d'un GPS. Les caractéristiques environnementales ont été définies avec l'aide des géographes du Bureau d'études et d'aménagement de l'urbanisme de Kinshasa, et ont servi de base aux fiches de renseignements 
individuels pour chaque piège. Nous avons saisi les informations concernant les facteurs présents ou absents de l'environnement du piège.

L'objet statistique était le point de capture et les variables discrètes et dichotomiques (présence/absence) ; la démarche a d'abord consisté à vérifier la solidité des groupes ( $5 \%$ mini.); puis à comparer les variables individuelles à l'intérieur des groupes retenus (test du Chi carré) et rechercher les interactions possibles (régression

Tableau 1.

Facteurs environnementaux et humains décrivant les points de capture de glossines inclus dans l'enquête entomologique conduite à Kinshasa en 2005

\begin{tabular}{|lll|}
\hline $\begin{array}{l}\text { Facteurs } \\
\text { environnementaux }\end{array}$ & Types & Modalités \\
\hline Sols & $\begin{array}{l}\text { Argileux, sablo-argileux, } \\
\text { sableux }\end{array}$ & \\
\hline Végétation & $\begin{array}{l}\text { Recrù forestier, } \\
\text { verger, savane arborée, } \\
\text { savane herbeuse, } \\
\text { champs vivriers, }\end{array}$ & $\begin{array}{l}\text { Présence ou non } \\
\text { de cultures }\end{array}$ \\
\hline jardins maraîchers, steppe & \\
\hline Eau & $\begin{array}{l}\text { Rivière, ruisseau, étang, } \\
\text { marécage, } \\
\text { point d'approvisionnement } \\
\text { en eau (puits, source) }\end{array}$ & $\begin{array}{l}\text { Régime permanent } \\
\text { Distance du point } \\
\text { d'eau au point } \\
\text { de capture }\end{array}$ \\
\hline Activités liées à l'eau & $\begin{array}{l}\text { Puisage, lavage de produits } \\
\text { agricoles, lavage domestique } \\
\text { (vaisselle, lessive, etc.), } \\
\text { baignade, carrière de sable, } \\
\text { lieu d'accostage de pirogues, } \\
\text { lieu de passage (pont, gué) }\end{array}$ \\
\hline Bovin, ovin, porcin, caprin, \\
avicole, pisciculture
\end{tabular}


logistique) entre facteurs biotiques et facteurs environnementaux (sols, végétation, présence d'eau) ou humains (activités). Considérant que les populations de glossines n'étaient pas les mêmes en saison sèche et en saison des pluies, les deux populations ont été analysées séparément.

\section{Des populations de glossines à faible effectif se maintenant grâce au couvert arboré et à la présence d'humidité}

Les résultats obtenus par les deux enquêtes entomologiques et les analyses réalisées au laboratoire à l'université de Yaoundé-I (Cameroun) ont fourni nos variables dépendantes.

Le nombre de glossines capturées, évalué par la DAP, est très faible quelle que soit la saison et est inférieur à 1 glossine par jour, l'écart-type des captures entre les pièges est de 9,8. 570 des 897 des glossines capturées $(65,5 \%)$ ont été disséquées et retenues pour les analyses, parmi elles $54(9,4 \%)$ étaient des ténérales.

Tableau 2.

Résultats des enquêtes entomologiques menées

en saison des pluies (SP) et en saison sèche (SS) à Kinshasa en 2005

\begin{tabular}{|c|c|c|c|c|c|c|c|c|c|c|c|c|c|}
\hline \multirow{2}{*}{\multicolumn{2}{|c|}{$\begin{array}{c}\text { Pièges } \\
\text { n }\end{array}$}} & \multicolumn{2}{|c|}{$\begin{array}{l}\text { Glossines } \\
\text { capturées }\end{array}$} & \multirow[t]{2}{*}{$\begin{array}{l}\text { DAP } \\
\text { moy. }\end{array}$} & \multicolumn{2}{|c|}{$\begin{array}{l}\text { Glossines } \\
\text { disséquées }\end{array}$} & \multicolumn{2}{|c|}{$\begin{array}{l}\text { Glossines } \\
\text { ténérales }\end{array}$} & \multicolumn{2}{|c|}{ TBG } & \multicolumn{2}{|c|}{$\begin{array}{c}\text { Repas } \\
\text { de sang }\end{array}$} & \multirow{2}{*}{$\begin{array}{l}\text { RSH } \\
\text { n } \%\end{array}$} \\
\hline & & $n$ & $\%$ & & $n$ & $\%$ & & $\%$ & $n$ & $\%$ & & $\%$ & \\
\hline SP & 305 & 624 & 69,6 & 0,51 & 376 & 60 & 30 & 8 & 8 & 2,1 & 53 & 14 & 3566 \\
\hline SS & 305 & 273 & 30,4 & 0,22 & 194 & 71 & 24 & 12 & 5 & 2,6 & 64 & 33 & 4367 \\
\hline Tota & & 897 & 100 & 0,36 & 570 & 65,5 & 54 & 9.4 & 13 & 2,3 & 117 & 20,5 & 7866,6 \\
\hline
\end{tabular}

DAP moy. : densités apparentes moyennes de glossines par piège et par jour :

Glossines disséquées: proportion (\%) de glossines disséquées parmi la population de glossines capturées;

Glossines ténérales: proportion (\%) de ténérales parmi les glossines disséquées:

TBG, proportion (\%) d'intestins de glossines infectés par Trypanosoma brucei gambiense dans l'ensemble des glossines disséquées:

Repas de sang: proportion (\%) de glossines gorgées parmi les glossines disséquées:

$\mathrm{RSH}$ : proportion (\%) de repas de sang humain dans l'ensemble des repas de sang analysés. 
$117(20,5 \%)$ glossines analysées au laboratoire avaient un repas de sang frais, en proportion significativement plus importante en saison sèche (33\%). Parmi les 117 repas analysés, 78 avaient été pris sur l'homme (67\%; IC: $57,5-75,9)$ et 32 sur le porc $(27,3 \%$; IC: 19-36) et 7 non identifiés. La proportion de repas de sang humain pris en saison sèche est significativement supérieure (Khi ${ }^{2}$ Pearson, $\mathrm{p}<0,000$ ) à celui observé en saison des pluies. Enfin, 2,3\% (IC 95\%: 1,2-3,3) des intestins analysés étaient infectés par le pathogène.

Pour être plus exploitables sur un plan statistique nos données environnementales ont fait l'objet de regroupements présentés dans le tableau 3.

Les facteurs insuffisamment représentés ont été éliminés (ex.: "habitat dense", «élevage caprin/ovin », etc.); les deux sites où aucune glossine n'a été capturée pendant la première enquête entomologique de saison des pluies n'ont pas été réintégrés dans l'étude en saison sèche. Le type de sol le plus rencontré est sabloargileux $(63,9 \%$ des points de capture); la végétation de la périphérie est plutôt herbacée $(49,5 \%)$ et $56,4 \%$ des pièges ont été placés à proximité d'un cours d'eau. Par contre, 13,4\% des pièges comprenaient dans leur rayon d'action à la fois un cours d'eau et un point d'eau "stagnante". Concernant l'activité humaine, il y a autant de points de capture sans activité qu'avec une ou plusieurs. Parmi les 305 points, on notait la présence d'un élevage familial de porcs pour $16,7 \%$ d'entre eux. L'habitat humain était présent dans l'environnement de $41,3 \%$ des pièges.

L'observation des tableaux 4 et 5 nous confirme que les sols argileux, les cours d'eau et la végétation arborée constituent des facteurs environnementaux qui favorisent la présence du vecteur tout au long de l'année et ce, quelle que soit la saison. La végétation mixte semble constituer un refuge pour les tsé-tsé en saison sèche. Pour les ténérales, qui sont à la fois un indicateur de lieux de reproduction et un groupe sensible à l'infection, les lieux de prédilection sont les rives des cours d'eau en saison des pluies, et les aires couvertes de végétation mixte en saison sèche. Inversement, couvert herbacé et eaux stagnantes sont plutôt défavorables à la présence de ces jeunes glossines. Concernant le contact homme/vecteur, celui-ci apparaît comme étant localisé 
Tableau 3.

Regroupements des données environnementales et poids des groupes dans les 305 points de captures

retenus pour l'enquête entomologique à Kinshasa (2005)

\begin{tabular}{|c|c|c|c|}
\hline $\begin{array}{l}\text { Facteurs } \\
\text { environnementaux }\end{array}$ & Types & Regroupements & $\begin{array}{l}\text { Points } \\
\text { de } \\
\text { captures } \\
\text { n (\%) }\end{array}$ \\
\hline Sols & $\begin{array}{l}\text { Argileux, } \\
\text { sablo-argileux, } \\
\text { sableux }\end{array}$ & $\begin{array}{l}\text { Argileux } \\
\text { sablo-argileux } \\
\text { sableux }\end{array}$ & $\begin{array}{c}79(25,9) \\
195(63,9) \\
31(10,2)\end{array}$ \\
\hline \multirow[t]{3}{*}{ Végétation } & $\begin{array}{l}\text { Recrû forestier, } \\
\text { verger }\end{array}$ & $\begin{array}{l}\text { Végétation } \\
\text { arborée }\end{array}$ & $114(37,4)$ \\
\hline & $\begin{array}{l}\text { Savane, } \\
\text { champs vivriers, } \\
\text { jardins maraîchers, } \\
\text { steppe }\end{array}$ & $\begin{array}{l}\text { Végétation } \\
\text { herbacée }\end{array}$ & $151(49,5)$ \\
\hline & $\begin{array}{l}\text { Végétation arborée } \\
\text { + végétation herbacée }\end{array}$ & $\begin{array}{l}\text { Végétation } \\
\text { mixte }\end{array}$ & $40(13,1)$ \\
\hline \multirow[t]{4}{*}{ Eau } & $\begin{array}{l}\text { Absence } \\
\text { de point d'eau }\end{array}$ & $\begin{array}{l}\text { Absence } \\
\text { de point d'eau }\end{array}$ & $31(10,2)$ \\
\hline & Rivière, ruisseau & Eau courante & $172(56,4)$ \\
\hline & $\begin{array}{l}\text { Étang, marécage, } \\
\text { point } \\
\text { d'approvisionnement } \\
\text { en eau (puits, source) }\end{array}$ & Eau stagnante & $61(20)$ \\
\hline & $\begin{array}{l}\text { Eau courante } \\
+ \text { eau stagnante }\end{array}$ & Eau mixte & $41(13,4)$ \\
\hline $\begin{array}{l}\text { Activités } \\
\text { liées à l'eau }\end{array}$ & $\begin{array}{l}\text { Puisage, lavage de } \\
\text { produits agricoles, } \\
\text { lavage domestique } \\
\text { (vaisselle, lessive, etc.), } \\
\text { baignade, } \\
\text { carrière de sable, } \\
\text { lieu d'accostage } \\
\text { de pirogues, lieu de } \\
\text { passage (pont, gué) }\end{array}$ & $\begin{array}{l}0 \text { activité } \\
1 \text { activité } \\
2 \text { activités } \\
3 \text { et }+\end{array}$ & $\begin{array}{l}65(21,3) \\
84(27,5) \\
72(23,6) \\
51(27,5)\end{array}$ \\
\hline Élevage & $\begin{array}{l}\text { Bovin, ovin, porcin, } \\
\text { caprin, avicole, } \\
\text { pisciculture }\end{array}$ & $\begin{array}{l}\text { Présence d'un } \\
\text { élevage porcin }\end{array}$ & $51(16,7)$ \\
\hline Habitat & Dense, isolé & $\begin{array}{l}\text { Présence } \\
\text { d'au moins } \\
\text { un habitat }\end{array}$ & $126(41,3)$ \\
\hline
\end{tabular}


Tableau 4.

Exposition au risque de transmission de la THA

par les facteurs environnementaux en saison des pluies à Kinshasa (2005)

\begin{tabular}{|lllll|}
\hline $\begin{array}{l}\text { Facteurs } \\
\text { biotiques }\end{array}$ & $\begin{array}{l}\text { Facteurs } \\
\text { environnementaux }\end{array}$ & $\begin{array}{l}\text { Odds } \\
\text { ratio }\end{array}$ & $\begin{array}{l}\text { Intervalle } \\
\text { de confiance } \\
\mathbf{( 9 5} \mathbf{)})\end{array}$ & p \\
\hline Glossines & Argile & 2,77 & $1,64-4,69$ & 0,0001 \\
& Cours d'eau & 2,31 & $1,42-3,74$ & 0,0005 \\
& Couvert arboré & 1,79 & $1,11-2,87$ & 0,01 \\
\hline Ténérales & Cours d'eau & 3,37 & $1,23-9,21$ & 0,009 \\
& Couvert herbacé & 0,29 & $0,11-0,76$ & 0,006 \\
& Eau stagnante & 0,15 & $0,002-1,07$ & 0,015 \\
\hline Repas de & Cours d'eau & 2,5 & $1,04-5,98$ & 0,03 \\
sang humain & & & & \\
\hline
\end{tabular}

Tableau 5 .

Exposition au risque de transmission de la THA

par les facteurs environnementaux en saison sèche à Kinshasa (2005)

\begin{tabular}{|llllc|}
\hline $\begin{array}{l}\text { Facteurs } \\
\text { biotiques }\end{array}$ & $\begin{array}{l}\text { Facteurs } \\
\text { environnementaux }\end{array}$ & $\begin{array}{l}\text { Odds } \\
\text { ratio }\end{array}$ & $\begin{array}{l}\text { Intervalle } \\
\text { de confiance } \\
\mathbf{( 9 5} \% \mathbf{)}\end{array}$ & p \\
\hline Glossines & Argile & 2,53 & $1,45-4,44$ & 0,001 \\
& Couvert mixte & 2,38 & $1,19-4,77$ & 0,02 \\
& Cours d'eau & 2,02 & $1,16-3,51$ & 0,01 \\
& Couvert arboré & 1,86 & $1,09-3,16$ & 0,02 \\
\hline Ténérales & Couvert mixte & 4,07 & $1,42-11,72$ & 0,02 \\
& Eau stagnante & 0,00 & $0,00-0,30$ & 0,005 \\
\hline Repas de & Porc & 3,81 & $1,72-8,44$ & 0,002 \\
sang humain & Couvert arboré & 2,57 & $1,21-5,44$ & 0,01 \\
\hline
\end{tabular}

principalement le long des cours d'eau en saison des pluies, et à proximité des élevages porcins ou sous couvert arboré en saison sèche. Aucune interaction significative $(\mathrm{p}<0,05)$ n'a été observée entre les différents groupes. 


\section{Un risque de transmission lié à I'humidité mais aussi à une certaine pratique de l'élevage}

Cette étude a cherché à associer observations géographiques et analyses biologiques, permettant ainsi de relier la dimension moléculaire depuis les organes du vecteur (identification du pathogène ou du sang dans l'intestin du vecteur) jusqu'à une échelle plus large qui est celle du point de capture dans son environnement.

Glossina fuscipes quanzensis est l'unique espèce de glossine capturée. Les faibles densités de glossines observées, inférieures à une glossine par piège et par jour, confirment les observations réalisées par R. DE DEKEN et ses collaborateurs (2005) qui avaient remarqué que la distribution du vecteur n'avait pas ou peu évolué en périphérie de la ville depuis les enquêtes de M. KazUMBA et collaborateurs en 1993. De Deken et ses collaborateurs décrivirent aussi des populations à distribution fragmentée. Ces auteurs avaient expliqué les variations saisonnières à peine perceptibles, par les faibles amplitudes climatiques entre les deux saisons en raison des remontées de courants d'air frais du Benguela par le fleuve Congo qui adoucissent le climat équatorial de Kinshasa. Néanmoins, il serait dangereux de sous-estimer l'importance épidémiologique d'une population de glossines fragmentée et de faible densité. F.-P. Gouteux et M. Artzrouni (2000), utilisant une approche biomathémathique, sont parvenus à modéliser la résurgence de la maladie du sommeil et ont pu observer que les faibles densités de tsé-tsé, combinées à un important contact homme/vecteur pouvaient provoquer des flambées épidémiques dans la population humaine. Cette hypothèse a pu être vérifiée dans de nombreux foyers d'Afrique centrale et de l'Ouest. Kinshasa offre ici toutes les conditions permettant la survenue de telles flambées. Le taux moyen de ténérales obtenu dans les deux enquêtes est conforme aux proportions classiquement observées (LAVEISSIÈRE et al., 2000). Les fortes proportions de repas de sang pris sur l'homme (67\%) et sur le porc (32\%) en font les hôtes préférentiels en périphérie de la ville, en dépit de préférences 
trophiques éclectiques de $G$. f. quanzensis. Il est vrai que la pression anthropique très forte sur le milieu et sans doute à l'origine de la disparition de la faune sauvage a modifié le comportement alimentaire des glossines. La proportion de repas de sang humain significativement plus importante en saison sèche, nous indique clairement qu'en dépit d'une baisse relative des densités de populations de glossines, la saison sèche favorise un contact plus étroit entre l'homme et le vecteur. Les deux mammifères servant d'hôtes nourriciers, l'homme et le porc, sont ainsi des réservoirs potentiels pour le pathogène; il est donc peu surprenant de retrouver ce dernier dans les intestins de 2,3\% des glossines disséquées dans les deux enquêtes. La caractérisation moléculaire de T. b. gambiense est encore récente (HERDER et al., 2002) et n'a été que peu pratiquée sur le terrain, excepté en Côte d'Ivoire où un taux similaire a été observé (JAMONNEAU et al., 2004). Si ce taux n'est pas le reflet de la proportion de glossines réellement infectantes (présence du pathogène dans les glandes salivaires), qui est classiquement estimé à 0,2\% (FrÉZIL et CUISANCE, 1994), il n'en constitue pas moins une évidence, celle de la circulation du pathogène chez le vecteur dans une zone où l'hôte nourricier principal est l'homme.

Une exposition accrue au risque de transmission en présence de certains facteurs environnementaux est confirmée. Les sols argileux, les cours d'eau et la végétation arborée favorisent la présence du vecteur tout au long de l'année. La végétation mixte semble constituer un refuge pour les tsé-tsé en saison sèche. Pour les ténérales, qui sont à la fois un indicateur de lieux de reproduction et un groupe sensible à l'infection, les lieux de prédilection sont les rives des cours d'eau en saison des pluies et les aires couvertes de végétation mixte en saison sèche. S'il est difficile de tracer un schéma général de la dynamique des populations de glossines, le climat et la disponibilité des hôtes nourriciers sont les deux facteurs environnementaux les plus influants sur la longévité et la mortalité des adultes. Il est reconnu que la saison sèche entraîne toujours une chute des densités d'insectes: augmentation de la mortalité des pupes, éclosion des imagos sans réserves de graisse, raréfaction de l'eau et du couvert végétal, soit autant d'événements qui sont atténués par une humidité permanente le long des cours d'eau et dans les sols argileux, ainsi que des températures plus clémentes à l'ombre des couverts arborés (LAVEISSIÈRE et al., 2000). 
Le contact homme/glossine apparaît lui aussi comme étant localisé principalement le long des cours d'eau en saison des pluies, et à proximité des élevages porcins ou sous couvert arboré en saison sèche. Inversement, l'intensité de l'activité humaine ne semble avoir aucune influence sur ce contact. Les activités liées à l'eau observées dans cette étude sont pourtant des activités quotidiennes, excepté le lavage des produits agricoles qui peut être lié à un rythme saisonnier réglé sur les récoltes. Par contre, le couvert arboré favorise le contact homme/vecteur en saison sèche, offrant un lieu de repos à la fois pour les glossines et pour les hommes, la promiscuité qui s'ensuit est de toute évidence un facteur aggravant du risque. Cette variabilité du risque de transmission a déjà été mise en évidence dans un foyer de THA en zone forestière du SudCameroun (GRÉBAUT et al., 2004), où il fut trouvé que l'exposition au risque était beaucoup plus importante en saison sèche, en dépit d'une baisse significative de la densité de population du vecteur. De même, la proximité de porcs multiplie presque par quatre le contact entre homme et vecteur en saison sèche. Sachant que la plupart des porcheries rencontrées dans l'étude sont situées dans une pièce de l'habitation familiale consacrée à cette activité, c'est vraisemblablement la pratique spécifique de l'élevage porcin à Kinshasa qui renforce les interrelations entre les trois acteurs du complexe pathogène. Pourtant, au Cameroun, P. Grébaut et ses collaborateurs (2004) avaient remarqué que les glossines se nourrissaient plus ou moins sur les porcs en fonction des saisons, sans que cela n'influence significativement la proportion de repas de sang pris sur l'homme; mais l'étude se passait en zone forestière et les animaux sauvages jouaient le rôle d'hôtes régulateurs saisonniers. C'est ici qu'une observation plus pointue de l'activité humaine dans l'espace périurbain apporterait vraisemblablement un éclairage nouveau sur le contact entre l'homme et le vecteur.

\section{Conclusion}

Même si cette étude met en évidence une transmission active de la THA en périphérie de Kinshasa par l'identification des facteurs biotiques à risque et des biotopes favorables à ces mêmes facteurs, il n'en demeure pas moins que l'analyse des pratiques sociales et 
agricoles liées à l'occupation de l'espace en périphérie de Kinshasa fait défaut. Au-delà du résultat de l'étude biologique et géographique, qui ne visait qu'à répondre à la question de l'existence d'un foyer endémique local, de nombreuses interrogations se posent sur les pratiques humaines qui conduisent les Kinois à s'exposer involontairement dans les biotopes considérés comme étant à risque. On pourrait aussi se projeter dans l'avenir et chercher à modéliser l'évolution de l'endémie à moyen terme, compte tenu de l'évolution de la pression anthropique sur ce milieu. Mais cette étude dont la période de référence était limitée à une année se devait d'apporter des réponses rapides au PNLTHA de RDC, tout comme au bailleur de fonds. La nécessité de résultats à court terme a donc hypothéqué la puissance d'analyse d'une étude menée conjointement avec des sociologues et des anthropologues qui ne pouvaient se contenter du court terme pour mieux comprendre quelle relation les Kinois ont pu établir avec cette zone périurbaine qui module leur exposition à la THA.

\section{Références bibliographiques}

Bilengue C. M., Meso V. K., Louis F. J., LuCAS P., 2001 - Human African trypanosomiasis in the urban milieu, the example of Kinshasa, Democratic Republic of the Congo, in 1998 and 1999. Med. Trop., 61 (4-5) : 445-448.

CATTAND P., 2001 - The epidemiology of human African trypanosomiasis, a complex multifactorial history. Med. Trop., 61 (4-5) : 313-22.

Courtin F., Dupont S., Zeze D.G., Jamonneau V., Sane B., Coulibaly B., CUNY G., SOLANO P., 2005 - Human African trypanosomiasis, urban transmission in the focus of Bonon (Côte d'Ivoire). Trop. Med. Int. Health, 10 (4) : 340-346.

De Deken R., Sumbu J., Mplana S., Mansinsa P., Wat'senga F, Lutumba P., Boelaert M., Van Den Bossche P., 2005 - Trypanosomiasis in Kinshasa, distribution of the vector, Glossina fuscipes quanzensis, and risk of transmission in the peri-urban area. Med. Vet. Entomol., 19 (4): 353-359.

Ebeja A. K., Lutumba P., Molisho D., Kegels G., Miaka Mia Bilenge C., BOELAERT M., 2003 - Sleeping sickness in the region of the town of Kinshasa, a retrospective analysis during the surveillance period 19962000. Trop. Med. Int. Health, 8 (10) : 949-955. 
Fournet F., Kone A., Meda A. H., Traore S., Hervouet J.-P., 2001 Integration of demographic factors in the characterization of risk areas for sleeping sickness in Côte d'Ivoire. Med. Trop., 61 (4-5) : 372-375.

FRÉZIL J.-L., CUISANCE D., 1994 - Trypanosomiasis, diseases with future, prospects and uncertainty. Bull. Soc. Pathol. Exot., 87 : 391-393.

GouteuX J.-P., LANCIEN J., 1986 - The pyramidal trap for collecting and controlling tsetse flies (Diptera, Glossinidae). Comparative trials and description of new collecting technics. Trop Med. Parasitol., 37 (1) : 6166 .

GouteuX J.-P., ARTZROUNI M., 2000 - Persistence and resurgence of sleeping sickness caused by Trypanosoma brucei gambiense in historic foci. Biomathematical approach of an epidemiologic enigma. C. R. Acad. Sci. III, 323 (4) : 351-364.

Grébaut P., Mbida J. A., Kondjio C. A., Njiokou F., Penchenier L., LAVEISSIĖRE C., 2004 - Spatial and temporal patterns of human African trypanosomosis (HAT) transmission risk in the Bipindi focus, in the forest zone of southern Cameroon. Vector Borne Zoonotic Dis., 4 (3) : 230-238.

Herder S., Simo G., Nkinin S., NJiokou F., 2002 - Identification of trypanosomes in wild animals from southern Cameroon using the polymerase chain reaction (PCR). Parasite, 9 (4): 345-349.

Hervouët J.-P., LAVEISSIÈre C., 1987 - Les grandes endémies: l'espace social coupable. Politique Africaine (FRA), 28: 21-32.

Jamonneau V., Ravel S., Koffi M., Kaba D., Zeze D. G., Ndri L., Sane B., Coulibaly B., Cuny G., Solano P., 2004 - Mixed infections of trypanosomes in tsetse and pigs and their epidemiological significance in a sleeping sickness focus of Côte d'Ivoire. Parasitology, 129 (Pt 6): 693-702.

Kazumba M., Kazadi K., Mulumba M.-P., 1993 - Caractéristiques de la trypanosomiase de l'enfant : à propos de 19 observations effectuées au CNPP, cliniques universitaires de Kinshasa, Zaire. Ann. Soc. Belg. Méd. Trop., Déc., 73 (4) : 253-59.

LAVEISSIĖRE C., 1975 - Détermination de l'âge des glossines ténérales (Glossina tachinoides Westwood). Cah. Orstom, Sér. Entomol. Méd., 13 (1): 3-11.

Laveissière C., Grébaut P., Herder H., Penchenier L., 2000 - Les glossines vectrices de la Trypanosomiase humaine africaine. Paris, IRD Éditions, $246 \mathrm{p}$.

Lointier M., Truc P., Drapeau L., Nanga S., Tarek M., 2001 Methodology to determine risk zones for sleeping sickness in Côte d'Ivoire by the spatial approach. Med. Trop., 61 (4-5) : 390-6. 
Moser D. R., CoOK G. A., Ochs De., Bailey C. P., Mckane M. R., DONELSON J. E., 1989 - Detection of Trypanosoma congolense and Trypanosoma brucei subspecies by DNA amplification using the polymerase chain reaction. Parasitology, 99 (Pt 1) : 57-66.

Njiokou F., Simo G., Mbida Mbida A., Truc P., Cuny G., Herder S., 2004 - A study of host preference in tsetse flies using a modified heteroduplex PCR-based method. Acta Trop., 91 (2) : 117-120.

OMS, 2006 - Trypanosomiase humaine africaine (maladie du sommeil), mise à jour épidémiologique. Relevé épidémiologique hebdomadaire, 81 (8) : 71-80. http//www.who.int/wer/2006/wer8108.pdf

Penchenier L., Grébaut P., Eboo Eyenga V., Bodo J.-M., Njiokou F., Binzoul J.-J., Simarro P., Soula G., Herder S., Laveissière C., 1999 The focus of human trypanosomiasis in Campo (Cameroon). History and endemic situation in 1998. Bull. Soc. Pathol. Exot., 92 (3) : 185-190.

Robays J., Ebeja Kadima A., Lutumba P., Miaka Mla Bilenge C., Kande Betu Ku Mesu V., De Deken R., Makabuza J., Deguerry M., Van Der STUYFT P., BOELAERT M., 2004 - Human African trypanosomiasis amongst urban residents in Kinshasa, a case-control study. Trop. Med. Int. Health, 9 (8) : 869-875.

Van Nieuwenhove S., Betu-Ku-Mesu V. K., Diabakana P. M., DECLERCQ J., BILENGE C. M., 2001 - Sleeping sickness resurgence in the DRC, the past decade. Trop. Med. Int. Health, 6 (5) : 335-341. 



\section{Un regard d'historien sur la lutte contre le paludisme au Cameroun (1950-1960)}

Jean-Paul BADO

Le paludisme a constitué et continue d'être une menace pour la mise en valeur socio-économique des régions tropicales. Il a longtemps inquiété les administrations coloniales et celles issues des indépendances (SCHWEITZ, 1949; Comité OMS, 1974). Avec la découverte du Plasmodium en 1880 et l'identification de l'anophèle femelle en 1898, un pas important a été franchi. Ces succès renforçaient les travaux de Caventou et de Pelletier qui isolèrent en 1820 le principe actif du quinquina, et les essais réussis de François-Clément Maillot d'abord à Perpignan puis en Algérie en 1834 dans l'utilisation de la quinine comme médicament préventif et curatif. Enfin, dès le début du vingtième siècle, les observations des médecins coloniaux dont beaucoup de parasitologues avaient permis de ruiner la croyance en la prétendue «immunité des Noirs" aux manifestations malariennes. Les spécialistes comprirent le rôle des températures, de la place fondamentale de l'eau, en particulier de l'emplacement de l'habitat par rapport aux sources d'eau et de la gestion de l'eau. Il fallut attendre 1947 pour comprendre le cycle exo-érythrocytaire du 
parasite dans l'organisme humain. Il restait à connaître toute la bio-écologie des anophèles impliqués dans la transmission, c'est-àdire leurs habitats précis (lieux de repos, de reproduction), leurs habitudes alimentaires, leur espérance de vie, leur capacité de vol, ainsi que leurs rapports avec les êtres humains.

En raison de son climat de type équatorial pour la partie sud avec des pluviométries dépassant les 4000 millimètres d'eau par an et quatre saisons (deux pluvieuses et deux sèches), et de type soudanien pour la partie nord avec moins de 1000 millimètres d'eau et deux saisons (une sèche et une pluvieuse), le Cameroun fut le territoire idéal pour progresser dans la recherche en vue de comprendre tous les facteurs favorisant l'essor de la maladie (GRAMONT, 1947).

Au début des années 1950, les administrations coloniale et internationale ont favorisé l'essor des plantations d'arbres à quinquina (PeIRIER, 1942; Archives IMTSSA*, carton 232) dont on pouvait extraire la poudre de quinine dans le laboratoire de Dschang construit en 1940-1941. Les médecins recommandaient de munir les lits de moustiquaires et de protéger les ouvertures des maisons par des grillages métalliques aux mailles très fines. On disposait déjà des insecticides à effets rémanents, particulièrement le DDT, pour appuyer la politique de lutte défendue dès 1948 par l'OMS qui se fondait sur les réussites spectaculaires dans de nombreux pays aux caractéristiques différentes, particulièrement l'Italie, la Grèce, le Mexique, le Panama, les États-Unis et le Venezuela. En 1955, l'OMS se référant aux travaux de nombreux experts fixa comme objectif l'éradication du paludisme à l'échelle planétaire.

Nous aborderons ici la question de lutte contre le paludisme dans le Cameroun sous mandat à la fin des années 1940, et les différentes stratégies antivectorielles et antiparasitaires appliquées dès les années 1950 avec le concours des experts de l'OMS et de l'Orstom. Nous nous attacherons également à montrer les difficultés rencontrées dans la conception d'une politique de santé publique prenant en compte les questions d'assainissement au sens large, c'est-à-dire d'hygiène du milieu, au moment de l'indépendance du pays.

I * Institut de médecine tropicale du Service de santé des armées. 


\section{État de la question du paludisme au Cameroun avant les années 1950}

Avant les années 1950, les administrations coloniales qui se succédèrent dans le protectorat du Cameroun disposèrent de moyens réduits pour combattre le paludisme qui demeurait une maladie prioritaire dans les textes sans l'être dans la réalité. La prophylaxie engagée dépendait étroitement des priorités économiques coloniales. Bien que dévoreuses de nombreuses vies humaines, les symptômes de la parasitose étaient moins spectaculaires que ceux de la maladie du sommeil et de la fièvre jaune. Yaoundé et Douala, ainsi que leurs environs immédiats étaient réputés pour la fréquence des accès pernicieux, des atteintes de fièvre hémoglobinurique. Ces deux villes disposaient de service urbain d'hygiène et de voirie dont le rayon d'action était limité et sans effet. À l'hôpital de Yaoundé de 1945 à 1949, 15\% des malades européens y étaient admis pour des cas de paludisme. Il convient de noter que la partie méridionale de la ville possédait de nombreux étangs, le plus souvent artificiels, véritables gîtes à moustiques; l'eau s'accumulait en amont des routes qui barraient la vallée (FRANQUEVILle, 1968; ChAMPAUD, 1972). Douala, bâtie sur les rives du fleuve Wouri, était couverte de marécages et abritait une forte population flottante qui vivait dans des bidonvilles où régnaient la promiscuité et l'insalubrité (CALSAT, 1946). Quant aux malades autochtones, les statistiques sont peu fiables sur le taux d'infestation. On peut affirmer que beaucoup d'enfants décédaient avant l'âge de cinq ans en raison des conditions de vie difficiles et de la virulence des parasites, particulièrement le Plasmodium falciparum répandu dans la région.

Jusqu'en 1951, il y eut peu d'actions organisées dans les agglomérations urbaines contre les moustiques et leurs larves; la lutte contre le paludisme ne figurait pas parmi les priorités de l'administration coloniale. Au manque de moyens s'ajoutait l'absence de connaissances précises sur l'écologie des anophèles. Les autorités sanitaires se contentaient de la chasse aux gîtes à moustiques et de la séparation des habitats européens et africains. Par mesure d'hygiène est alors édictée une décision introduisant une ségrégation 
de fait dans la ville de Yaoundé; en 1923, toutes les cases des indigènes ou paillotes habitées par des Noirs ont été transférées hors du périmètre urbain ; la population européenne était invitée à ne pas laisser se former de villages indigènes aux alentours des maisons d'habitation (FRANQUEVILLE, 1968; CHAMPAUD, 1972). Il faut noter que certains médecins influents s'opposaient aux mesures de lutte en avançant que la maladie avait toujours existé sans jamais provoquer de catastrophes démographiques.

En dépit de cette désorganisation, les spécialistes biomédicaux poursuivirent leurs recherches pour confirmer la variation des infestations palustres qui dépendaient des régions où l'on se trouvait et des saisons. Ils purent établirent des zones et des périodes d'endémicité, d'épidémicité et de sporadicité du paludisme. Ces acquis aidèrent les autorités sanitaires, par ailleurs soumises aux pressions internationales, à faire finalement du paludisme une maladie dont il fallait s'occuper en priorité, conformément aux directives de l'OMS.

Le protectorat disposait d'atouts: d'une part, les structures du Service général d'hygiène mobile et de prophylaxie (SGHMP) avec sa section paludisme où collaboraient les chercheurs et techniciens entomologistes de l'Orstom et d'autre part, les plantations d'arbres à quinquina dont on extrayait les sulfates de quinine. Sur le plan international, l'essor des antimalariques de synthèse permettait de nourrir beaucoup d'espoirs, espoirs que venait encore renforcer la possession des insecticides à effet rémanent (DDT, dieldrine) utilisés avec succès en ltalie, puis en Corse. Il faut noter que sans rejeter la chimioprophylaxie par la "cure radicale» défendue par certains responsables médicaux, les autorités avaient opté pour l'usage des insecticides à effets rémanents tout en reconnaissant le risque d'accidents en cas de réimportation d'anophèles infestés (Archives IMTSSA, carton 108). Le Cameroun, comme le reste de l'Afrique française, bénéficia des subsides du Fonds d'investissement pour le développement économique et social (Fides), des aides du Fonds des Nations unies pour l'enfance (Fise), et de la contribution des experts paludologues de l'OMS et de l'Orstom.

Jusqu'en 1950, il n'existait pas de "médicament idéal " tel que le Comité d'experts du paludisme de l'OMS l'envisageait, à savoir "être un bon agent thérapeutique, et, en même temps capable de 
réaliser une guérison radicale; avoir une faible toxicité; pouvoir être facilement obtenu à un prix [réduit]» (Archives IMTSSA, carton 108).

À la veille de la décision d'appliquer à l'échelle du protectorat une véritable lutte antipaludique, les autorités camerounaises améliorèrent les trois anciennes stratégies pour combattre efficacement la maladie: la "prophylaxie offensive», souvent qualifiée de guerre aux moustiques et centrée sur les gîtes anthropiques, et la «prophylaxie défensive» qui s'articule autour de la protection des individus contre les piqûres des arthropodes. La "prophylaxie médicamenteuse" constituait la troisième stratégie; elle permettait de traiter les malades jusqu'à tarir les parasites chez les porteurs sains ou chez les malades.

\section{Les années 1950 et les nouvelles stratégies de lutte contre le paludisme}

Le directeur de la santé au ministère des Colonies, le médecin inspecteur Vaucel, traça les grandes lignes à suivre dans la nouvelle stratégie de lutte fondée sur l'utilisation des insecticides à effets rémanents à combiner à la lutte chimioprophylactique. L'Afrique française devait s'inspirer des travaux du malariologiste A. Gilroy du service de santé du Nigeria de passage à Dakar pour former les responsables de l'A.-O. F. (Chronique d'Outre-mer, 1951; BADO, 2004). De plus, les autorités camerounaises reçurent de la mission Jaugeon-Trinquier des consignes nécessaires pour réussir leurs campagnes antipaludiques, en particulier connaître les comportements des moustiques impliqués dans la propagation des parasites. La mission préconisait d'appliquer le système développé par le professeur Missiroli dans les marais Pontins, puis en Corse, et qui différenciait les anophèles endophiles et exophiles (Chronique d'Outre-mer, 1951; BADO, 2004). Cependant pour l'Afrique et les régions tropicales, Ronald Ross avait mis en garde contre ce schéma "de mœurs endophiles et exophiles " en raison des comportements exo-endophiles de certaines espèces et 
dont il fallait tenir compte dans les campagnes de pulvérisations. Laction répulsive du DDT devait également être prise en compte. Aussi, les autorités sanitaires étaient invitées à considérer le matériau de construction des habitations pour évaluer l'importance de l'imprégnation des insecticides et la durée de la rémanence: les maisons bâties avec du banco (boue séchée au soleil), surtout dans le nord du Cameroun, n'avaient pas la même capacité d'absorption des produits insecticides que celles fabriquées avec des parpaings ou du bois et des roseaux. La hauteur des plafonds devait également être prise en compte dans le programme de pulvérisation. Enfin, se posait le problème du recrutement de la main-d'œuvre qualifiée et des salaires qui devaient être attractifs en raison de la pénibilité du travail et surtout de la concurrence du secteur privé où les ouvriers étaient mieux payés.

Le plan élaboré en 1949 qui concernait l'Afrique équatoriale française et le Cameroun, ne fonctionna, en fait, qu'à partir de 1953 par manque de moyens financiers et de personnels suffisamment formés. Ce plan-programme était financé par la France à travers le Fides, par l'OMS avec la contribution d'experts, et par le Fise avec la fourniture de la logistique (moyens de transport, pompes, insecticides; et éventuellement médicaments chimioprophylactiques) (CHASTANG, 1959). Il a été conduit successivement par les docteurs Morin et Najera et, depuis, 1957 par le professeur Livadas. Simultanément aux campagnes d'aspersion, les spécialistes de l'Orstom et les experts de l'OMS menèrent des recherches pour une meilleure identification des espèces anophéliennes présentes et impliquées dans la transmission de la parasitose, le Cameroun se chargeant du budget de fonctionnement. Les premières campagnes de masse débutèrent au nord dans la région du Diamaré pour protéger 390000 personnes. Les campagnes au sud commencèrent au cours de la même année 1953, précédées comme au nord d'enquêtes relatives aux anophèles impliqués et suivies par des house-spraying.

Sur les quinze espèces d'anophèles répertoriées, seules quatre furent reconnues intervenir activement dans la transmission du

1 En l'absence d'expression française équivalente et aussi concise, ce terme anglo-saxon désigne l'application des insecticides à effet rémanent sur des surfaces intérieures des habitations. 
Plasmodium. Il s'agit des Anopheles gambiae, funestus, moucheti et nili. Les travaux de Livadas, Mouchet, Gariou et Chastang ont permis de localiser géographiquement ces espèces sur l'ensemble du Cameroun (Archives IMTSSA, carton 233, Paludisme AOF-AEF). Déjà présent au nord en raison de ses besoins d'endroits déboisés et ensoleillés, à proximité des habitations, pour s'épanouir et se reproduire, A. gambiae a pénétré dans les régions forestières camerounaises à la faveur des activités humaines liées aux déforestations et aux plantations. Cette espèce, qui n'était pas fréquemment rencontrée dans les villages du Cameroun méridional avant les campagnes de désinsectisation, était toutefois au contact direct des populations des agglomérations urbaines où elle était le principal vecteur du paludisme. A. funestus était également présent sur le territoire en particulier à Douala envahie par une forte population flottante. Des recherches menées dans le sud et l'est du pays n'ont rien donné, ce qui permit de conclure qu'il n'est pas non plus un moustique forestier. Ainsi que A. gambiae, il a investi les régions fortement urbanisées et dégradées, notamment les grandes agglomérations. Néanmoins, sa faible présence le fait considérer comme un vecteur secondaire du paludisme. En ce qui concerne $A$. moucheti, il est reconnu dans les gîtes naturels de la zone forestière où il demeure le véritable vecteur du paludisme dans la partie méridionale. Dans les villages prospectés et fortement impaludés, les chercheurs ont constaté sa seule présence. A. nili largement répandu au nord et dans toute l'Afrique tropicale, a été reconnu dans le sud du Cameroun où les recherches ont permis d'identifier ses larves dans presque tous les cours d'eau. Il est toutefois reconnu comme un vecteur secondaire du paludisme en raison de sa présence réduite dans les habitations.

Simultanément à l'élaboration de la cartographie des espèces anophéliennes, les chercheurs ont identifié des facteurs favorisant les contacts entre les êtres humains et les anophèles pour mieux diriger les campagnes d'aspersions intra- et extra-domiciliaires. Le fait de passer la nuit dans les habitations, de conserver de l'eau dans les récipients non couverts et de vivre dans un environnement marécageux contribuait fortement à l'impaludation. Les moustiques se cachaient souvent dans les habitations mal éclairées et dotées de peu d'ouvertures. Les entomologistes et les parasitologues s'aperçurent que la faune anophélienne variait en quantité et en qualité d'un village à l'autre. Ils découvrirent que certaines localités 
étaient infestées par un seul vecteur soit $A$. nili, soit $A$. moucheti. Les localités infestées par $A$. gambiae seul étaient surtout les grandes villes. Souvent, deux espèces occupaient le même espace où elles se relayaient dans la transmission en fonction des saisons. On s'était aperçu lors des travaux d'assainissement des marécages où pullulait $A$. gambiae, que les larves de cet insecte disparaissaient et étaient remplacées par celles de A. funestus (Archives IMTSSA, Carton 232, Documents techniques). L'endémie était alors déterminée par l'importance des vecteurs.

Au cours de ces travaux préliminaires, les enquêteurs soulignèrent l'impact des températures sur la persistance de la maladie, les températures élevées favorisant l'accomplissement du cycle sporogonyque des parasites toute l'année. Ces enquêtes épidémiologiques contribuèrent à mettre en cause l'administration coloniale dans la création de nouveaux foyers palustres et l'extension de certaines espèces anophéliennes, particulièrement $A$. gambiae, dans des régions où elles n'existaient pas auparavant.

Outre les informations obtenues sur le moustique anophèle, les enquêteurs purent identifier les principales victimes du paludisme et qui participaient à son développement: les enfants, en particulier ceux âgés de zéro à cinq ans, les femmes enceintes, les personnes déjà fragilisées par d'autres affections, et les résidents de fraîche date. Les auteurs de ces enquêtes épidémiologiques se sont aperçus aussi que malgré la possession des anticorps antipaludiques par la majorité des habitants quel que fût leur âge, le taux de morbidité augmentait avec l'arrivée de la saison des pluies qui favorisaient la pullulation des moustiques et le taux élevé de piqûres par individu. En somme, les populations camerounaises payaient cher cette situation par la forte morbidité et mortalité chez les enfants, et avec des adultes soumis aux incessantes piqûres des anophèles, souvent en proie à des fièvres invalidantes qui affectaient leur rendement au travail.

\section{Les campagnes de lutte}

Le mot d'ordre des campagnes était de frapper vite et fort les anophèles dans les centres urbains en anéantissant leurs larves et 
les lieux de reproduction. De ces centres et de leurs environs immédiats, l'action devait progressivement s'étendre aux régions rurales. L'OMS avait estimé qu'il fallait procéder par étapes à cause des spécificités des comportements des anophèles, des conditions climatiques et surtout de l'immensité des zones impaludées. Des zones pilotes (la région du Diamaré, au nord, et la région de Yaoundé) servirent de laboratoires d'expérimentations. Les travaux dans ces deux zones devaient permettre de tester les techniques à utiliser lors des phases dites de pré-éradication puis d'éradication en vue d'une extension à grande échelle des campagnes de pulvérisation et de chimioprophylaxie avec les antimalariens de synthèse qui supplantaient la quinine (Archives IMTSSA, carton 108, Cameroun rapport 1951-1955).

La cartographie des espèces aida à délimiter les zones à traiter avec des insecticides. Yaoundé et Douala qui bénéficiaient des services d'hygiène urbaine eurent peu de difficultés à recruter du personnel. Ces deux localités, en tant que telles, étaient exclues des zones pilotes d'expérimentation. Il restait à former les manœuvres aux méthodes de pulvérisation et à l'entretien du matériel. À Maroua, au nord, des campagnes d'aspersions furent réalisées en 1953 pour détruire A. funestus, le vecteur le plus répandu. Les saisons bien contrastées (saison sèche et saison pluvieuse) facilitaient le fait de pouvoir déloger les anophèles qui se réfugiaient dans les maisons à la saison sèche. D'autres campagnes de pulvérisation furent engagées jusqu'en novembre 1954 à Garoua et à Ngaoundéré. Dans ces campagnes de pulvérisation au nord, de mai à août 1954, il y eut 260382 personnes protégées, 243164 cases aspergées au DDT (dont 40000 mètres carrés de locaux publics) équivalant à 8880 mètres carrés de surface traitée avec $32,735 \mathrm{~kg}$ de produit. À l'ouest, ce furent les agglomérations de Foumban, Foubout, Bangangté, Mbouda, Dschang, Bafang, Bafoussam et Nkongsamba. À la fin de l'année 1954, les équipes de pulvérisation avaient réussi à protéger 327185 personnes, en traitant 13725447 mètres carrés de surface avec 50144 tonnes de DDT. Les 13725447 mètres carrés étaient composés de 12943263 mètres carrés de cases africaines, de 435452 mètres carrés de cases européennes. Les 346732 mètres carrés restant étaient constitués de locaux divers. De 1954 à 1957, il y a eu dans le Diamaré huit cycles semestriels d'aspersions au DDT. Ainsi 
que le nota le chef de la Section paludisme du Cameroun en 1959, les résultats obtenus ne furent pas négligeables puisque les indices hématologiques se virent abaisser au niveau de l'hypo-endémie dans les villes et dans les villages de la brousse au taux d'une méso-endémie modérée (CHASTANG, 1959).

Ainsi qu'il a été souligné précédemment, la zone pilote de la région de Yaoundé (à l'exception de la ville elle-même) créée en 1953 se limitait à une région habitée par près de 50000 habitants autour de Yaoundé. Cette zone d'expérimentation s'étendit en permettant de protéger 70000 personnes. Les conditions climatiques (abondantes précipitations, humidité constamment élevée, températures chaudes avec de faibles écarts) étaient très favorables, d'autant que la région était traversée d'est en ouest par trois cours d'eau importants (la Sanaga, le Nyong et le Ntem). À l'est coulaient la Kadéi, la Boumba et le Dja avec leurs nombreux affluents. Répartie en trois secteurs A, B et C, cette zone pilote de Yaoundé fut soumise aux séances d'aspersion au DDT, à la dieldrine et au $\mathrm{HCH}$. Le chef d'équipe était secondé par un entomologiste et un épidémiologiste. Les aspersions d'insecticides commencées à la fin février 1953 partirent de la périphérie vers le centre de la zone en suivant dix axes qui convergeaient vers Yaoundé (Archives IMTSSA, carton 233, Cameroun AOF-AEF).

Il faut retenir, aussi bien au sud qu'au nord, que la fréquence des opérations d'aspersions avaient fini par provoquer des réticences chez de nombreux habitants qui ne supportaient plus d'être dérangés, qui ne percevaient pas le danger du paludisme, et qui n'étaient guère impliqués dans la lutte antipaludique. De 1954 à 1956-1957, les campagnes d'aspersion conservèrent un caractère fragmentaire, ne parvenant pas à traiter tous les villages, ni à atteindre tous les habitants. Seuls 50 à $60 \%$ de la population étaient protégés ce qui ne suffisait pas à assurer une sécurité. Ces conclusions décevantes obligèrent les responsables des campagnes d'aspersion à réorganiser la zone pilote autour de Yaoundé à partir de mars 1957 (fig. 1).

Ils l'agrandirent en l'étendant d'est en ouest sur 60 kilomètres et du nord au sud sur 100 kilomètres afin de mettre le maximum de populations à l'abri, soit 120000 habitants de zones rurales. Cette nouvelle zone regroupait trois catégories de villages. Il y avait les 
Figure 1.

Zone pilote de Yaoundé.

Carte extraite des Archives IMTSSA, carton 233, Cameroun, A.-O.F. A.-E.F,, légèrement modifiée par l'auteur.
Zone Pilole de Yoounde
A Erig Région trailée depuis 1956
B $\iota_{-} \exists$ Region trailée depuis 1955
C $\square$ Région Ir ailée pour la première fois en 1057
D Station temoin non traitée
_Limite entre les secteurs traités à la Dieldrine (est) el ou D.D.T. (ouest)

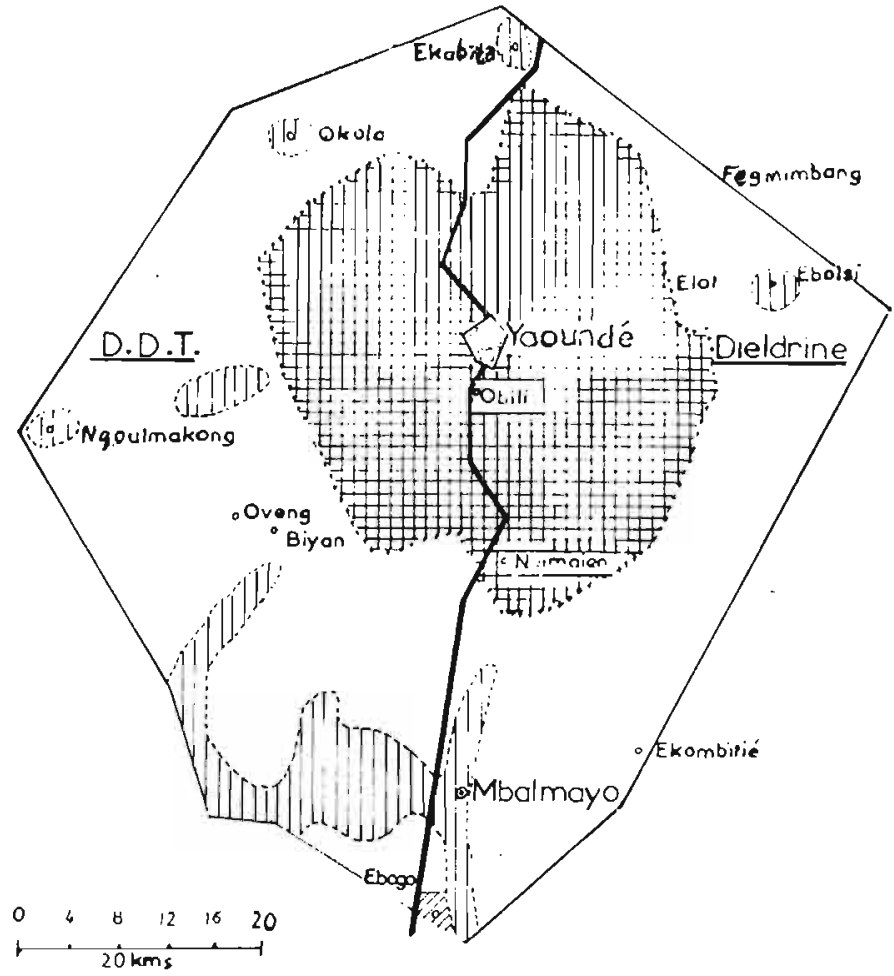

villages traités depuis 1954, 1955 et 1957 et des villages témoins conservés pour évaluer la pertinence de l'effet des insecticides à effets rémanents. Afin d'anticiper les échecs, du moins pour éviter de mauvaises répartitions de la pellicule des produits insecticides, les responsables s'imposèrent des mesures draconiennes à euxmêmes et aux manœuvres affectés aux aspersions: avoir une connaissance précise de la région en dépit de la tension politique extrême du moment, contrôler quotidiennement les équipes 
d'aspersions sur le terrain pour les contraindre à entrer dans toutes les agglomérations et tous les hameaux, même les plus difficiles d'accès, effectuer surtout des tournées de rattrapage pour traiter les habitations trouvées fermées lors du premier passage. Il ne fallait en aucun cas laisser échapper des anophèles susceptibles de coloniser de nouveau les zones traitées et retransmettre la maladie.

Depuis sa réorganisation, la zone pilote reçut un traitement complet de mai à juin 1957, conformément aux recommandations du Comité d'experts de l'OMS qui reconnaissait qu'en Afrique, la transmission était si intense que des méthodes appliquées avec succès ailleurs y devenaient inefficaces. Il fallait donc traiter toutes les habitations et opérer des pulvérisations avec le plus grand soin et au bon moment dans toute la zone considérée (Archives OMS MHO/AS/52.57). Le DDT a servi de janvier à avril 1958; la dieldrine de mars à avril de la même année. La période d'aspersion fut ramenée à dix mois au lieu d'un an en raison de constatations entomologiques. On parlait déjà dans d'autres régions de résistance des anophèles aux insecticides. Dans la zone pilote de Yaoundé, puis du sud (en y englobant la zone forestière), les responsables des aspersions avaient pris des précautions pour prévenir les cas de résistances.

Dans la partie A de la zone, il y eut les campagnes de pulvérisations de 1954 à 1956, puis un traitement complet en 1958; la transmission du paludisme y avait pratiquement disparu. Dans le secteur B traité depuis 1955-1956, les aspersions complètes ont permis de réduire considérablement le taux de transmission. Le paludisme y était sur le point d'être éliminé après les campagnes de 1958. Quant au secteur C, les indices malariométriques avaient fortement baissé après les campagnes de 1958.

Outre les aspersions, des engins de terrassement procédaient aux grands travaux d'assainissement des espaces par le creusement de canaux de drainage des eaux de pluies dans les centres urbains, ou bien à leur nettoyage avant le passage des équipes d'aspersion. Des manœuvres engagés devaient parcourir chaque secteur avec leur pompe à main pour asperger les moindres flaques d'eau, ainsi que tous les endroits où des objets pouvaient se transformer en gîtes à moustiques. Le travail d'aspersion commençait le lundi matin pour se terminer le samedi à midi. 
Dans la partie sud du pays, en particulier dans la zone pilote, A. gambiae n'avait aucune chance de survivre en raison de l'environnement de plus en plus défavorable à son épanouissement. Par contre, dans la partie nord du Cameroun, la variété des gîtes le rendait plus difficile à atteindre. Quant à $A$. moucheti, il ne constituait plus un danger véritable puisque l'objectif de la lutte était de tarir sa source d'approvisionnement en parasites en le repoussant le plus loin possible des hommes pour mettre un terme à la transmission. Depuis 1955, A. nili n'avait plus été capturé. Néanmoins, en 1958, les équipes de recherche avaient récolté de nombreux cadavres à Nyabessan (Ntem) à l'entrée des maisons traitées. Même si $A$. nili ne constituait plus une menace de la transmission dans la partie sud-camerounaise, il fallait rester vigilant à cause de l'importance des déplacements des habitants et surtout du rôle que pouvait jouer la population flottante dans l'essaimage des parasites malariens. Cette partie du territoire était sécurisée jusqu'en 1958. Aucun phénomène de résistance aux insecticides n'y avait été découvert si bien que les chercheurs soulignaient qu'on pouvait raisonnablement éradiquer le paludisme du Cameroun à condition de résoudre les difficultés administratives et surtout de trouver les financements.

Au cours de l'année 1958, les enquêtes et contre-enquêtes de parasitologues, d'entomologistes et de cliniciens autorisèrent à conclure que la situation palustre à Yaoundé qui comptait 56000 habitants est aussi favorable que possible (CHASTANG, 1959; HAMONG et al., 1963). Il était exceptionnel de trouver des hématozoaires dans un frottis de sang. Dans les très rares cas positifs, les porteurs de parasites s'étaient contaminés à l'extérieur de la zone; on peut donc affirmer que dans la ville de Yaoundé la transmission du paludisme est totalement interrompue (Archives IMTSSA, carton 233). Déjà dès 1955, on n'y trouvait plus trace de larves et d'imago de $A$. gambiae en dépit de minutieuses recherches. Par contre à Douala, ville portuaire, capitale économique et principale centre commercial, peuplée de 120000 personnes en 1958, la situation n'était pas satisfaisante malgré un traitement complet qui avait entraîné la disparition de A. gambiae. Sa présence dans les villages voisins avait permis une ré-invasion de la ville à la faveur des pluies abondantes et d'un ralentissement des opérations d'aspersions. Des prospections organisées dans le quartier de Kassalafan permirent de capturer 
de nombreux adultes $A$. gambiae. Douala souffrait de son statut qui attirait une forte population flottante difficile à contrôler et qui y introduisait de nouvelles normes de gestion de l'espace dans le paysage urbain. Par exemple les populations du Nord laissaient des trous liés à l'extraction de la terre pour bâtir des maisons en banco. Ces trous se transformaient en gîtes à moustique avec les chutes de pluies. Le fait d'agrandir la ville par des lotissements pour faire reculer la brousse et éloigner les taudis, laissait présager cette ré-invasion. Du reste, tout comme à Yaoundé, dans certains quartiers de Douala, les habitants avaient des activités qui facilitaient l'invasion des moustiques, tels l'élevage de porcs et le développement des jardins potagers. De plus, à la veille de l'indépendance du pays, certains hommes politiques pour des raisons électorales, contournaient des directives dérangeantes mais néanmoins nécessaires pour un contrôle effectif du paludisme.

À l'indépendance en 1960, les équipes de campagne arrêtèrent les aspersions après le contrôle des experts de l'OMS et soumirent la zone pilote à une surveillance épidémiologique. Ces succès devaient être exploités dans d'autres régions du pays. Néanmoins, le phénomène de résistance tant redouté se précisa dans l'arrondissement de Nanga-Eboko fin 1959. Les A. gambiae étaient devenus insensibles au traitement à la dieldrine. L'OMS alertée prit des dispositions pour tenter de contrer la capacité de résistance qui commençait à s'étendre en proposant d'associer la distribution de chloroquine aux aspersions de la dieldrine. La pyriméthamine seule avait déjà été écartée en raison de la résistance observée en 1956. Au nord, aucune action anti-anophélienne n'avait réussi à faire baisser de façon durable après 1958 le niveau d'endémicité. Il fut alors décidé de proposer une lutte médicamenteuse. En novembre 1960, à l'exception des villes de Yaoundé et de Douala, les indices malariométriques remontèrent dans tous les groupes d'âge de la population. L'inefficacité du DDT déjà observée dans cette zone fit choisir aussi la lutte chimioprophylactique contre la parasitose surtout à destination des écoliers. Les actions entreprises auprès de ces derniers devaient amener à convaincre toute la population de se conformer à ce nouvel essai. Ainsi que le souligna un paludologue de l'OMS invité en 1966 à donner son avis sur le plan de chimioprophylaxie proposé au Cameroun, l'une des causes principales d'échecs de tous les essais et de tous les projets pilotes, 
dans diverses régions africaines, a été la sous-estimation du besoin de la collaboration active de la population, dans le cadre de l'éradication. Même cette nouvelle tentative de contrôle de la maladie par l'intermédiaire des écoliers souffrait de nombreux défauts surtout en période de vacances où les enfants se retrouvaient dans leur famille sans médicaments.

\section{Conclusion}

La tentative de contrôle du paludisme au Cameroun à travers les zones pilotes a d'abord été un franc succès jusqu'au début des années 1960 en rendant l'environnement défavorable aux différentes espèces d'anophèles. Mais cette victoire fut de courte durée en raison de l'immensité des espaces, de l'insuffisance de contrôle des populations aux habitudes diverses, particulièrement dans la gestion de leurs habitats et de leurs habitations, ainsi que dans la compréhension de la maladie. À cela s'est ajouté l'essor des phénomènes de résistance des anophèles aux insecticides DDT et dieldrine, et la chimiorésistance à la pyriméthamine et à l'association prémaline-pyriméthamine, ce qui jeta un trouble parmi les experts paludologues.

En voulant contrôler la maladie, les experts ont omis de prendre en considération les perceptions culturelles du paludisme mais aussi les pratiques dans les espaces de vie. Ce déficit dans l'analyse a constitué un important obstacle au contrôle de la maladie si bien que dès la découverte de l'ampleur des résistances, il était trop tard pour rectifier les erreurs et surtout pour s'opposer à l'arrêt des campagnes pilotes après l'interdiction par les experts de l'OMS de se servir des insecticides dont les scientifiques n'avaient pas anticipé le court délai d'efficacité.

Face aux problèmes croissants, les nouvelles autorités du Cameroun n'eurent d'autre choix que d'arrêter les campagnes d'aspersion d'insecticides, de développer la prophylaxie médicamenteuse et de proposer aux habitants des villes un modèle d'habitation qui devait permettre de vivre à l'abri des piqûres des anophèles. Son coût élevé découragea les personnes susceptibles 
d'être intéressées. Aussi, les modèles proposés, malgré la prise en compte des impératifs sanitaires, ne cadraient pas avec les habitudes des habitants. Ainsi que le reconnut en 1967 un expert: Dans un programme de pré-éradication du paludisme, mises à part les tâches de développement des services de santé de base pour réaliser la couverture totale du pays, la tache principale est de concentrer tous les efforts sur la protection de l'homme, c'est-à-dire faire l'éducation sanitaire, l'assainissement du milieu et assurer la protection médicamenteuse, avec comme but baisser le niveau du paludisme le plus bas possible afin d'assurer la prévention contre la mortalité et l'invalidité causées par cette maladie. Cette question non résolue au début des années 1960, se pose toujours aux populations africaines en proie au paludisme. Il serait intéressant de tirer des leçons de ces luttes entreprises au Cameroun en tenant compte des rapports étroits qui existent entre médecine préventive, santé publique, assainissement au sens large et milieu de vie.

\section{Références bibliographiques}

BADO J.-P., 2004 - La lutte contre le paludisme en Afrique centrale: problème d'hier et d'aujourd'hui. Enjeux, bulletin d'analyse géopolitique de l'Afrique centrale, $\mathrm{n}^{\circ} 18,10-14$.

BADo J.-P., 2005 - « Malarial Campaigns in Francophone Africa from the 1940s: A Challenge for Medicine $»$. In Ngalamulume K., Viterbo P.: Colloque Health and Medicine in Africa, Brin Mwarn, avril.

CALSAT J. H., 1946 -Enquête monographique pour le projet d'urbanisme de la région du Wouri et de Douala, sl du Gouvernement du Cameroun français. 155 feuillets ronéo.

Champaud J., 1972 - Genèse et typologie des villes du Cameroun Ouest. Cahiers Orstom, série Sciences humaines, vol. IX, $n^{\circ} 3$ : 325-336.

Chastang R., 1959 - Quatre années de lutte antipaludique au Cameroun méridional. Médecine tropicale, vol. 19, 1:51-86.

Chronique d'Outre-mer, 1951 - La lutte contre le paludisme en Afrique française d'après le rapport de la conférence internationale du paludisme 29 nov./7 décembre 1950, octobre: 11-16.

Comité OMS d'experts du paludisme, 1974 - Seizième rapport OMS. Série Rapport techniques, $\mathrm{n}^{\circ} 549$. 
Franqueville A., 1968 - Le paysage urbain de Yaoundé. Cahiers d'Outre-Mer, avril-juin: 113-154.

GRAMONT Dr J. L., 1947 - Le paludisme. Marchés tropicaux, nov. : 16791683.

Hamong J., Mouchet J., Chauvet G., Lumaret R., 1963 - Bilan de quatorze années de lutte contre le paludisme dans les pays francophones d'Afrique et de Madagascar. Considérations sur la persistance de la transmission et perspectives d'avenir. Bulletin de la Société de Pathologie exotique, Filiales, 56: 933-971.

Peirier C., 1942 - Note sur le quinquina du Cameroun. Médecine tropicale, $\mathrm{n}^{\circ} 2: 99-119$.

SCHWEITZ J., 1949 - Le quatrième Congrès international de Médecine tropicale et de paludisme. Zaïre, vol. III, $\mathrm{n}^{\circ} 1$, janvier: 35-45. 



\section{La perception du risque palustre chez les femmes dans deux villes camerounaises: Maroua et Bafoussam}

\section{Estelle KOUOKAM MAGNE}

$\mathrm{Au}$ Cameroun, le paludisme reste la première cause de mortalité et de morbidité, et comme ailleurs, il touche principalement les jeunes enfants et les femmes enceintes, malgré les découvertes médicales et les différentes stratégies mondiales mises en œuvre pour prévenir et (ou) guérir cette maladie. Pour A. SAME EkoBo (1997), deux raisons essentielles expliquent cette situation: le relâchement, sinon l'abandon de mesures d'assainissement, les pratiques thérapeutiques inadaptées (associations médicamenteuses, complexité des posologies) avec pour corollaire le développement des souches résistantes.

Dans les pays endémiques, les politiques de lutte contre le paludisme préconisent la prise en charge précoce de tout accès de fièvre. En Afrique tropicale, le diagnostic de cette maladie chez l'enfant à partir de la fièvre reste ambigu dans la mesure où ce symptôme se retrouve dans la plupart des maladies infantiles (diarrhée, infections respiratoires, etc.). Ainsi, la fièvre fait partie de l'expérience ordinaire des populations (GRUÉNAIS, 2003), et n'est pas spécifique au paludisme. Au cours des années 1960, la plupart 
des populations africaines ont été les cibles de campagnes de sensibilisation au risque palustre. À cet effet, les différents moyens de prévention (chimioprophylaxie, utilisation des moustiquaires imprégnées, pulvérisation des gîtes larvaires) ont été utilisés. De nos jours, les messages de lutte contre le paludisme destinés aux populations reposent sur le présupposé que, dans les perceptions populaires sur la transmission de la maladie, "la triade fièvrepaludisme-moustique» est admise et intégrée dans les conduites sociales de prévention. De ce point de vue, une amélioration des connaissances et des pratiques relatives à cette triade comme la promotion auprès des populations des moustiquaires imprégnées permettrait de contrôler l'endémie palustre et de "faire reculer le paludisme». En fait, pour connue qu'elle soit, cette triade fièvrepaludisme-moustique fait souvent figure de plus petit dénominateur commun de la rencontre entre connaissances populaires et savoir médical et s'inscrit en fait à l'intersection d'expériences issues de registres très disparates (GRUÉNAIS, 2003).

L'étude que nous avons réalisée $e^{1}$ avait pour principal objectif d'analyser et de comprendre comment les populations construisent le risque palustre en rapport avec leur environnement. En abordant le risque comme un objet "socialement construit » (BIBEAU, 1996: 27), il s'agissait pour nous de resituer le paludisme dans l'expérience quotidienne des populations en prenant en compte les relations qu'elles effectuaient entre la survenue des accès fébriles et la configuration de leur environnement.

L'étude que nous avons menée tend à montrer que les connaissances autour du paludisme relèvent d'une construction étiologique qui allie différents savoirs, spécialisés et profanes, auxquels vient s'ajouter une mémoire de la lutte contre le paludisme au Cameroun. Les populations disposent ainsi d'un «stock» de connaissances qui se renouvellent au rythme des messages de prévention, et les savoirs se constituent alors à partir de l'accumulation, de la sélection et de la hiérarchisation d'informations reçues. Parfois, seuls certains éléments de ce "capital de connaissances » sont pris en compte lors de la survenue d'un accès fébrile chez les enfants.

1 Étude réalisée dans le cadre du projet «Fièvres, prévention et perceptions de l'environnement au Cameroun», Projet $n^{\circ} 03.2 .431$ (2003-2005), ATC Santé Environnement, Fonds national de la science. 


\section{Les contextes d'étude}

La recherche s'est effectuée de juillet à octobre 2003 à Maroua, dans la province de l'Extrême-Nord du Cameroun, qui se situe dans une zone à transmission saisonnière, et de février à avril 2005 à Bafoussam, à l'ouest du pays, situé dans une zone à transmission continue. Nous avons rencontré près de 200 personnes, en majorité des mères de famille. En effet, ce sont elles qui prodiguent des soins aux enfants et sélectionnent les signes qui leur permettent de savoir que "l'enfant ne va pas bien ". Les pères ${ }^{2}$ jouent surtout un rôle financier dans le dispositif thérapeutique.

Les enquêtes se sont déroulées dans les services de pédiatrie des hôpitaux provinciaux de Bafoussam et Maroua, ainsi qu'au domicile des mères préalablement rencontrées dans ces services; par rapport aux catégories socio-professionnelles représentées dans cette étude, nous nous sommes référés aux métiers déclarés par nos interlocutrices. Les mères interrogées déclarent pour la plupart ( $n=160$ ) s'occuper des tâches ménagères, agricoles et de petit commerce; les autres $(n=40)$ sont soit institutrices, soit infirmières. Ces activités principales sont aussi accompagnées d'activités qu'elles présentent comme secondaires: répétition à domicile pour les institutrices, soins en clientèle privée pour les infirmières, agriculture et petits commerces.

Les entretiens semi-directifs ont été réalisés dans les différentes langues véhiculaires propres aux deux zones d'étude. À Maroua, les principales langues utilisées étaient le fulfulde, l'anglais et le français. Bien que Maroua soit une ville poulophone, plusieurs groupes ethniques y vivent avec des langues différentes. Dans sa pratique quotidienne, le fulfulde fait l'objet d'une réappropriation qui se traduit par « une créolisation linguistique» (BOTTE et al., 1999: 31). Les termes ne sont pas définis une fois pour toutes, malgré les récurrences, il n'y a pas d'homogénéité dans les manières de dire la maladie. Chez les interlocuteurs utilisant le fulfulde uniquement comme langue d'échanges et d'affaires, nous avons aussi pris en compte la dénomination du paludisme dans leur propre langue.

2 Nous ne nous limitons pas aux pères biologiques mais aux personnes de sexe masculin qui se sont présentés comme «pères», et qui apparaissent dans le processus de décision de prise en charge. 
À Bafoussam, quatre langues ont été employées: le ghomàlà, le pidgin english, l'anglais et le français. La prise en compte des différentes manières de dire la maladie dans les langues véhiculaires dans ces deux villes montre que dans une culture populaire donnée, les représentations de la maladie et de la santé sont pour une part largement partagées, même si le sens précis des mots peut varier d'un locuteur $\grave{a}$ un autre. Ces variations, qu'elles soient de type idiosyncrasique ou statutaire (homme/femme, aîné/cadet, guérisseur/profane), ne mettent pas en cause lintercommunicabilité des notions et conceptions véhiculées par ces mots (OlIVIER DE SARDAN, 1999: 11).

\section{Maroua et Bafoussam}

Marouaest le chef-lieu de la province de l'Extrême-Nord ${ }^{3}$. Sa population est estimée à 335831 habitants ${ }^{4}$. L'économie est basée essentiellement sur l'agriculture et l'élevage qui subissent les aléas climatiques. De nombreux cours d'eaux saisonniers traversent la ville. Le climat est de type soudano-sahélien avec une longue saison sèche ( 8 à 9 mois) et une courte saison des pluies ( 3 à 4 mois). En saison des pluies, on assiste à une bouffée saisonnière meurtrière pour les jeunes non immuns (...) la transmission est intense, bien qu'elle se poursuive toute l'année (RIPERT et PAJOr, 1996: 156). Le paludisme est la première cause de décès (37\%) chez les enfants de 0 à 5 ans $^{5}$. Ici, les éléments déterminants dans le diagnostic familial du paludisme sont la durée de la fièvre et les céphalées. D'où les appellations du paludisme en fulfulde: fabboore, pabboje, fabboje, traduites habituellement par «paludisme». Fabboore vient du verbe fabbugo: qui veut dire durer longtemps. Il se différencie de oppere qui est la fièvre (KovokAM MAGNE, 2004).

3 Avec une population estimée à 2628623 habitants et une densité de 77 habitants $/ \mathrm{km}^{2} .70 \%$ de cette population est rurale.

4 Population estimée sur la base des calculs effectués par World Gazetteer, 2006.

5 Délégation provinciale de la santé de la province de l'Extrême-Nord, Rapport d'activités, 2003. 
Bafoussam est le chef-lieu de la province de l'Ouest ${ }^{6}$. Sa population est estimée à 305053 habitants. Les populations de cette province (plus de 70\%) vivent essentiellement des activités agro-pastorales. Le relief est montagneux. Le climat est de type équatorial. L'année compte quatre saisons: une grande saison pluvieuse qui va de juin à octobre, suivie par la grande saison sèche, de fin octobre à mi-mars. La petite saison pluvieuse (mi-mars, mi-avril) la sépare de la petite saison sèche qui ne dure qu'un mois environ (mi-avril à fin mai). Une étude réalisée en 2004 montre que le paludisme est la première cause de mortalité $(36,8 \%)$ chez les enfants de 0 à 5 ans, et que seulement $20 \%$ des enfants atteints de paludisme/fièvre ont bénéficié d'une prise en charge correcte (ATEUCHIA et al., 2004).

Faire un "palu " c'est avoir des céphalées et une hyperthermie. En pidgin english, fippa, déformation du terme anglais fever, est utilisé pour nommer le paludisme. La plupart des langues utilisées dans la zone reprennent le même terme avec quelques altérations fewa, feppa... Quand nos enquêtées nomment le paludisme dans leurs langues respectives, elles précisent toujours: Ce n'est pas vraiment le patois. Mais c'est comme ça qu'on appelle. l'hyperthermie et les céphalées peuvent être associées à d'autres maladies: otites, diarrhée, pneumonie... La poussée dentaire est aussi répertoriée par les mères comme l'un des facteurs favorisant les accès fébriles. Selon les contextes, la fièvre chez les petits enfants n'est pas toujours assimilée au paludisme.

\section{Les savoirs sur la transmission de la maladie}

Les savoirs locaux sur la transmission de la maladie sont tributaires des configurations climatiques et environnementales propres à nos sites d'étude. En effet, les étiologies populaires sur le paludisme s'articulent autour de la saisonnalité, et des ordures qui favorisent le développement des moustiques. 
À Maroua, le paludisme est souvent considéré comme la maladie de la fraîcheur. À ce titre, le moustique n'est pas perçu comme l'unique vecteur du paludisme. Le climat (la fraîcheur due à la saison des pluies), la consommation de fruits et légumes frais et sucrés qui poussent en saison de pluies, ainsi que la consommation exagérée du lait de vache frais sont également rendus responsables du paludisme.

Ainsi, Adama, grand-mère de Salim âgé de 39 mois, évacué d'un centre de santé pour une anémie paludéenne nous confiait: On dit chez nous, les Fulbe, que c'est quand on boit du lait frais qu'on attrape le palu. Et les moustiques peuvent causer le palu. Pour le cas présent de cet enfant, je crois que c'est le lait. On en a bu (beaucoup) ces derniers temps. Pour Asta, mère de Mairiskou âgée de 7 mois: C'est l'eau de la pluie qui cause (le paludisme), quand la pluie te mouille, la fraîcheur empêche le sang de circuler et tu attrapes alors le palu.

À Bafoussam, le paludisme est considéré par les mères comme une maladie banale, mais elles ont constaté une fréquence importante des accès fébriles chez les enfants en période de transition climatique. Ainsi, elles en parlent aussi comme d'une maladie due au "changement de climat". Dans les conceptions populaires, la consommation des mangues cueillies pendant les premières pluies de l'année (mois de mars) favoriserait la survenue des accès fébriles.

Les perceptions du risque palustre s'inscrivent aussi dans une mémoire sociale de la lutte contre le paludisme. En effet, la lutte contre le paludisme a été associée à l'assainissement urbain, avec l'intervention des services d'hygiène dans les sphères publiques (les routes, les rues) et dans les sphères privées (maisons d'habitations) pour la destruction des gîtes anophéliens. Selon J.-P. BADO (2004), le développement des services d'hygiène entre 1955 et 1960 a favorisé une diminution importante du nombre de cas de paludisme dans certaines localités du Cameroun. Ces mesures d'hygiène publiques sont accompagnées de mesures de rétorsion. Ceux qui les transgressent risquent une amende ${ }^{7}$. Les personnes 
ayant vécu cette période la présentent de manière idyllique, et la dimension coercitive de ces différentes actions publiques n'est guère critiquée. Nos interlocutrices les plus âgées (une soixantaine d'années) ont la mémoire de cette époque qui se situe avant les années 1970, où il n'y avait pas de moustiques, par rapport à la période des années 1990 où les moustiques ont augmenté, tandis qu'au cours des années 1980, il y avait (des moustiques) mais pas comme maintenant. Pour illustrer combien la situation était «mieux avant», Élise parle de Douala, ville aujourd'hui reconnue pour l'importance des nuisances dues aux moustiques: Même à Douala! J'habitais New-Bell, il n'y avait pas de moustiques. Les bennes à ordures passaient tous les jours à $6 \mathrm{~h}$. Le palu, c'est d'abord une question d'hygiène! L'abandon, ou la faible implication des municipalités dans les mesures de salubrité contribuent à l'émergence d'un sentiment de colère chez nos enquêtés qui voient dans la forte transmission du paludisme l'un des indices du renoncement des pouvoirs publics à protéger les populations contre cette maladie.

Quelle que soit la ville (Maroua ou Bafoussam), le discours sur les moustiques est étroitement lié à celui sur l'eau, les ordures ou la saleté. Cette conception s'appuie sur une mémoire sociale de l'hygiène construite à partir de réinterprétations et de réappropriations des mesures hygiénistes que les populations ont connues et qui n'existent plus. Cette référence à l'environnement n'est pas nouvelle dans la construction sociale des risques pathogènes. En effet, l'histoire sociale de la lutte contre les maladies montre que deux éléments importants ont structuré les perceptions populaires de la transmission: les humeurs et les conceptions du propre et du sale. Les comportements sanitaires des populations ont été pendant longtemps influencés par leurs perceptions humorales (Vigarello, 1999; Corbin, 1986). Les représentations sociales du propre et du sale (VIGARELLO, 1985), du sain et du malsain, et leur continuelle redéfinition par les acteurs ont ainsi permis de mettre en ouvre des stratégies préventives et des pratiques de prévision (Vigarello, 1999). Les deux études de cas évoquées ci-dessous viennent illustrer comment sont ressentis les liens entre environnement domestique, nuisances et fièvres. Nos interlocuteurs parlent généralement d'un environnement «sale» qui favorise le "développement des moustiques». 
Djibril est chauffeur de moto à Maroua. Il vit dans une chambre louée avec sa femme qui est enceinte, sa belle-sœur et ses deux filles âgées respectivement de deux ans et demi et neuf mois. Il habite dans la même concession que son bailleur ainsi que d'autres locataires. La cour principale qu'il nomme un cimetière de voitures est remplie de carcasses d'automobiles rouillées qui appartiennent à son bailleur, qui en prélève les pneus ou toute autre pièce encore utile pour la vente. Il y a des arbres au milieu de la cour. Il arrive souvent que des pneus se remplissent d'eau après les pluies. La poubelle est dans un coin de la cour.

La chambre de Djibrilest en face de l'espace de la cuisine utilisé par toutes les femmes de la concession. Elle est couverte de tôles parsemées de trous qui laissent entrevoir de fins rayons de soleil. Quand il pleut, il doit souvent déplacer le lit. Les femmes dorment sur un matelas à côté du lit que Djibril partage avec ses deux filles. Le lit est couvert d'une moustiquaire d' " une place et demie» que sa belle-mère lui a offerte. Cette moustiquaire a été achetée au Nigeria $^{8}$ à 2500 FCFA. Sa concubine et sa sœur utilisent une bombe insecticide et dorment sur un matelas sans moustiquaire. Il n'y a pas de chaise dans sa chambre, le lit sert aussi de siège pour lui-même et ses invités. La natte est utilisée par les femmes quand il s'agit de faire la cuisine. La moustiquaire est généralement pliée au-dessus du lit et c'est au moment de se coucher ou de mettre les enfants au lit qu'elle est déployée. Quand elle est utilisée, elle n'est pas bordée.

Dans le cas de Djibril, la moustiquaire est un cadeau qui contribue à son confort personnel et à celui de ses enfants en bas âge. Elle fait partie de son standing, de son confort, dans la mesure où depuis qu'elle est là, il peut dormir, les moustiques ne dérangent plus. Pour Djibril, ce qui provoque l'excès de moustiques dans la concession, c'est «le cimetière de voitures qui peut cacher pire que le moustique, les serpents", ainsi que l'abattoir de moutons qui se trouve à côté de sa maison et dont l'odeur du sang pourri qui engraisse les moustiques et tous les déchets attirent les moustiques. En saison de pluies c'est pire, l'eau reste là. 
Grace est institutrice à Bafoussam. Ses enfants Jeanine et Blaise ont été hospitalisés pour paludisme. Au départ, ils ne dormaient pas sous la moustiquaire. J'ai quand même fait l'effort d'en acheter depuis près d'un an. J'ai constaté que ça a un peu diminué les fréquences de fièvre parce que avant j'étais à l'hôpital tous les deux mois internée, avec l'un ou l'autre. Pendant cette année, j'ai fait l'hôpital trois fois toujours pour la fièvre. Cette fois-ci, je me suis demandée où est alors le rôle de la moustiquaire? Vous voyez, ils sont tous les deux internés ici depuis samedi! Tous deux dorment sous la moustiquaire. La goutte épaisse est positive. Il y a un cours d'eau au fond qui descend. Il y a les moustiques, nous-mêmes nous allons jeter la poubelle là-dedans. C'est très grave et en saison de pluies, quand il y a déjà les eaux stagnantes un peu partout. C'est une petite source. Tout le quartier jette la poubelle dedans. C'est de là que proviennent les moustiques. Avec les rigoles partout! Les eaux stagnantes! Nous ne sommes pas épargnés! nous confie-t-elle.

Un lien est bien établi entre le paludisme et l'environnement immédiat, c'est-à-dire l'air respiré, les ordures et la saleté aux alentours de la maison. Ce sont surtout les nuisances sonores des moustiques qui empêchent de dormir qui sont mises en avant. Elles viennent parfois justifier l'usage de la moustiquaire dans certains cas. Tout se passe comme si, parfois, le moustique et les quelques éléments de connaissance médicale qui transparaissent dans les conceptions populaires venaient se surajouter à une mise en accusation globale des conditions de vie (GRUÉNAIS, 2003) puisque, comme on peut le rappeler, le vecteur du paludisme est un insecte silencieux et qu'il ne peut se reproduire que dans des points d'eau relativement propres.

À partir de leurs savoirs sur la transmission du paludisme, nos interlocuteurs mettent en œuvre des stratégies pour éviter la maladie. Mais, elles s'inscrivent dans la logique de la pratique, à laquelle il faut reconnaître (...) une logique qui n'est pas celle de la logique pour éviter de lui demander plus de logique qu'elle ne peut en donner et se condamner ainsi soit à lui extorquer des incohérences, soit à lui imposer une cohérence forcée (BOURDIEU, 1980: 144). Lorsque l'on observe les pratiques sociales de prévention, elles sont marquées par le besoin de propreté de l'environnement immédiat, de l'amélioration de l'espace de vie, et de la prévention 
d'autres affections dues à la toxicité de certains moyens de lutte antivectorielle $e^{9}$. En effet, il peut aussi arriver que les mères ayant des moustiquaires n'utilisent pas les bombes insecticides à cause de leurs effets sur la santé des enfants. Ces conceptions du propre et du sale, ainsi que celles relatives à la protection de soi ne rejoignent pas les normes de prévention préconisées par les programmes de lutte contre le paludisme.

\section{De l'expérience à l'action: les pratiques populaires de prévention}

Les mesures préventives adoptées par les acteurs ne s'inscrivent pas dans une logique linéaire qui allierait le discours à l'action. On peut constater un décrochage entre les pratiques et les discours (JAFFRÉ, 2003: 495). Même si les acteurs répertorient un ensemble d'éléments, les stratégies de prévention obéissent à une logique de hiérarchisation des risques qui s'inscrit dans la temporalité et prennent en compte les effets constatés de la survenue d'accès fébriles chez les enfants.

La "fraîcheur ", généralement citée par nos interlocuteurs, renvoie à l'humidité et au froid. Elle fait partie des éléments contre lesquels les parents protègent leurs enfants. Djaratou, mère de Dairou âgé de 15 mois, déclarait: Pendant la période de fraîcheur, c'est bien sûr le palu qui dérange le plus et comme cette année il y a eu trop de pluies, le palu a menacé vraiment mes enfants (...) Je leur donne de la nivaquine, et ils mettent un pull c'est tout ce que je fais pour prévenir le palu.

Les mesures de protection sont d'abord orientées vers les nuisances nocturnes, le paludisme vient ensuite. Il s'agit surtout, d'une amélioration du confort domestique et de la qualité du sommeil.

9 L'utilisation des bombes insecticides par exemple exige des précautions d'emploi qui ne sont pas toujours respectées par les mères : pulvériser dans la chambre et attendre pendant un certain nombre d'heures à l'extérieur pour que le gaz se dissipe. 
Pour se protéger des moustiques, divers moyens sont employés: la propreté de la maison, les bombes et spirales insecticides, les moustiquaires, la fermeture des portes et fenêtres au crépuscule, les plantes fumigènes.

Dans les conceptions populaires du propre et du sale, il arrive que l'environnement domestique soit présenté comme un micro-espace préservé des moustiques. Il n'est pas alors perçu comme appartenant à l'environnement global qui, lui, est considéré comme propice au développement des anophèles. Pour Adama, la grand-mère de Salim âgé de 3 ans et trois mois, la fermeture des portes et des fenêtres permet d'éviter les piqûres de moustiques: Il y a trop de moustiques dans mon quartier mais pas dans ma maison, car j'ai ma stratégie: je ferme toujours ma chambre et je n'allume pas la lumière durant cette période de moustiques. Comme ça, on ne peut pas trouver un seul moustique dans ma chambre.

Une autre alternative dans la lutte contre les nuisances des moustiques est l'utilisation des bombes insecticides. Pendant la saison des pluies, on peut entendre à la station radio provinciale, des publicités de marques de bombes et de spirales insecticides. Les annonceurs insistent sur l'importance des bombes insecticides dans l'élimination des moustiques et des acariens. Mais le mode d'emploi n'est pas toujours respecté par les mères.

Haoua, la mère de Bouba âgé de 4 ans que nous avons rencontrée pendant son séjour d'hospitalisation à Maroua, habite un quartier traversé par un cours d'eau. Elle est la première femme d'un ménage polygame. Elle a trois enfants. Elle explique l'absence de moustiquaire par le coût de celle-ci et la non-utilisation d'insecticides parce que ça fait le rhume aux enfants. Mais, dit-elle, je ventile seulement avec le pagne avant d'entrer et je chasse les moustiques de la chambre, c'est tout.

Ainsi, des méthodes de prévention peuvent être financièrement accessibles aux mères, mais elles peuvent présenter des inconvénients sur la santé des enfants et dans ce cas aussi, elles ne sont pas utilisées. En ce qui concerne l'usage des bombes insecticides, on peut constater que celles-ci ne sont pas employées par certaines mères en raison de leur nocivité. Il y a une hiérarchisation des risques qui s'opère: il s'agit d'éviter la toxicité dont les effets sont 
immédiatement observables chez les enfants (toux, rhume, etc.). Dans l'adhésion ou la non-adhésion aux mesures de prévention, une large part est faite aux conséquences immédiatement observables sur la santé des enfants.

\section{Conclusion}

La confrontation des savoirs des populations à leurs pratiques de prévention du paludisme nous a permis de constater des "décrochages » (JAFFRÉ, 2003) entre les discours et les pratiques populaires. Mais ces décrochages entre discours et pratiques nous permettent de revisiter la constitution des savoirs des populations dans le cadre des maladies tropicales à vecteurs en général et du paludisme en particulier. En effet, ces savoirs se construisent à partir de l'accumulation, la hiérarchisation et l'appropriation de savoirs profanes et spécialisés issus d'expériences passées et actuelles. Les stratégies populaires de prévention du paludisme font l'objet de réajustements, en fonction des environnements géo-climatiques, économiques et des différents messages de santé publique destinés aux populations.

Les savoirs sur la transmission et les pratiques de prévention du paludisme sont basés sur l'expérience et la connaissance de divers événements qui surviennent avant les accès fébriles chez leurs enfants. Ils constituent « un ensemble relativement organisé de pensée" (GEERTZ, 1986: 95). Les différents aspects que nous avons soulignés montrent que ces savoirs ne doivent pas être considérés comme des «attitudes» ou des « croyances». Ils s'inscrivent dans une logique pragmatique où une grande part est faite à l'expérience.

La recherche que nous avons effectuée montre l'importance d'une approche interdisciplinaire dans les recherches qui portent sur les problèmes de santé publique, et que l'apport d'études anthropologiques n'est pas juste «intéressante» (HEGGENHOUGEN, 2000). Lanthropologie intervient à un double niveau dans le cadre de la recherche sur cette maladie tropicale à vecteur: restituer toute la complexité des comportements des acteurs, qui se rapportent à leurs zones écologiques et à leurs conditions socio-économiques, 
et contribuer à repenser les messages de communication destinés aux populations. Elle trouve toute sa place dans un dialogue fécond entre disciplines (l'histoire, la santé publique, l'épidémiologie, etc.) pour une approche globale de la lutte contre le paludisme qui incluerait non seulement les données épidémiologiques mais aussi les particularités du milieu de vie des populations auxquelles s'adressent les campagnes, pour les adapter à leurs contextes. Le cas du Cameroun, pays caractérisé par une diversité bioclimatique, montre que la prise en compte du milieu dans les campagnes de prévention et de lutte contre le paludisme est primordiale.

\section{Remerciements}

Nous exprimons nos sincères remerciements à nos interlocuteurs/rices de Maroua et Bafoussam, au Programme national de lutte contre le paludisme du Cameroun ainsi qu'aux différentes délégations provinciales.

\section{Références bibliographiques}

Ateuchia P., Tigang, Fopa Amadou, 2004 - Rapport d'enquête de collecte des données de base pour le suivi et l'évaluation des activités de lutte contre le paludisme dans la province de l'Ouest. 50 p.

BADO J.-P., 2004 - La lutte contre le paludisme en Afrique centrale. Problèmes d'hier et d'aujourd'hui. Enjeux, nº 18, janvier-mars: 10-13.

BIBEAU G., 1996 - «La spécificité de la recherche anthropologique sur le sida ». In Benoist J., Desclaux A. (dir.): Anthropologie et sida, bilan et perspectives, Paris, Karthala, coll. Médecines du Monde, 13-30.

Botte R., Boutrais J., Schmitz J. (dir.), 1999 - Figures peules. Paris, Karthala, coll. Hommes et Sociétés, 539 p.

BOURdieU P., 1980 - Le sens pratique. Paris, Éditions de Minuit, coll. Le Sens Commun, 475 p.

Corbin A., 1986 - Le miasme et la Jonquille, l'odorat et l'imaginaire social, XVIII ${ }^{e}$ XIX ${ }^{e}$ siècles. Paris, Flammarion, coll. Champs Histoire, $336 \mathrm{p}$.

GeERTz C., 1986 - Savoir local, savoir global: les lieux du savoir. Paris, PUF, coll. Sociologie d'aujourd'hui, 293 p.

GRUÉNAIS M.-É., 2003 - Personnels de santé et populations face à l'expérience ordinaire des fièvres en Afrique subsaharienne. Médecine Tropicale, $\mathrm{n}^{\circ} 3$, vol. $63: 271-275$. 
HEGGENHOUGEN H. K., 2000 - More than just 'interesting' : anthropology, health and human rights. Social Science and Medecine, $\mathrm{n}^{\circ} 50$ : 1171-1175. JAFFRE Y., 2003 - «Transmission, prévention et hygiène en Afrique de l'Ouest, une question anthropologique de santé publique ». In Jaffré Y., Bonnet D. (dir.): Les maladies de passage, Paris, Karthala, coll. Médecines du Monde, 485-505.

KOUOKAM MAGNE E., 2004 - Une maladie qui dure: perceptions du risque palustre et recours therapeutiques lors des accès fébriles chez les jeunes enfants à Maroua (province de l'Extrême-Nord, Cameroun). Marseille, École de hautes études en sciences sociales, 103 p.

Olivier De SARDAN J.-P., 1999 - "Introduction ". In Jaffré Y., Olivier de Sardan J.-P. (dir.): La construction sociale des maladies. Les entités nosologiques en Afrique de l'Ouest, Paris, PUF : 7-12.

Ripert C., Pajot F.-X., 1996 - «Paludisme». In Ripert C., Pajot F.-X., Vincendeau P., Esquerdo Gomez F. (dir.): Epidémiologie des maladies parasitaires t. I, Cachan, Éditions Médicales internationales: 69-180.

SAme Еково A., 1997 - Santé, climat et environnement au Cameroun. Yaoundé, Jutey-Sciences, $328 \mathrm{p}$.

Vigarello G., 1985 - Le propre et le sale. L'hygiene du corps depuis le Moyen Âge. Paris, Seuil, coll. Points Histoire, 282 p.

Vigarello G., 1999 - Histoire des pratiques de santé. Le sain et le malsain depuis le Moyen Âge. Paris, Seuil, coll. Points Histoire, 390 p.

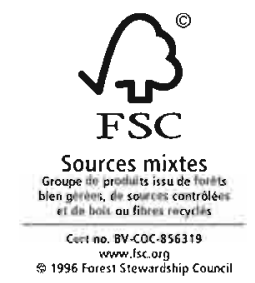

Imprimé en France. - JOUVE, 1, rue du Docteur Sauvé, 53100 MAYENNE N 539242S. - Dépôt légal : août 2010 
es domaines de la santé et de l'environnement ont longtemps été considérés, tant par les décideurs que par les scientifiques, comme des réalités disjointes. II a fallu attendre les dernières décennies pour que soit reconnu et que se concrétise le lien entre environnements et santé avec la création d'agences spécialisées.

Qu'il s'agisse de foyers de maladies parasitaires ou de pollution atmosphérique, les situations d'exposition à un risque sanitaire lié à l'environnement relèvent de facteurs particuliers : épidémiologiques, géographiques, politiques, et bien évidemment sociaux.

À partir de zones géographiques situées tant au Sud qu'au Nord, les auteurs de cet ouvrage présentent un large éventail de configurations dans lesquelles s'exprime la complexité des relations entre santé et environnements dans leur rapport avec les groupes humains. Ils mettent l'accent sur l'importance de la perception et de la pratique des acteurs (des décideurs aux bénéficiaires des mesures, en passant par les médecins) pour penser le risque sanitaire selon les milieux.

Ils soulignent tout l'intérêt de l'apport des sciences sociales et des pratiques interdisciplinaires dès lors qu'il s'agit d'envisager les relations entre un pathogène et l'homme. Chacune des approches s'attache ainsi à éclairer les différentes facettes du risque environnemental, depuis le comportement des individus jusqu'aux politiques nationales, en invitant le lecteur à se départir de toute vision déterministe et simplificatrice.

IRD

44, bd de Dunkerque

13572 Marseille cedex 02

editions@ird.fr

www.editions.ird.fr

Diffusion

IRD

32, av. Henri-Varagnat

93143 Bondy cedex

diffusion.bondy@ird.fr

\section{$\mathrm{S}_{\mathrm{E}} \mathrm{H}$}

Société d'Écologie

$38 €$

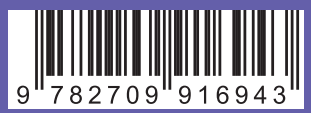

ISBN 978-2-7099-1694-3

ISSN 1958-0975 